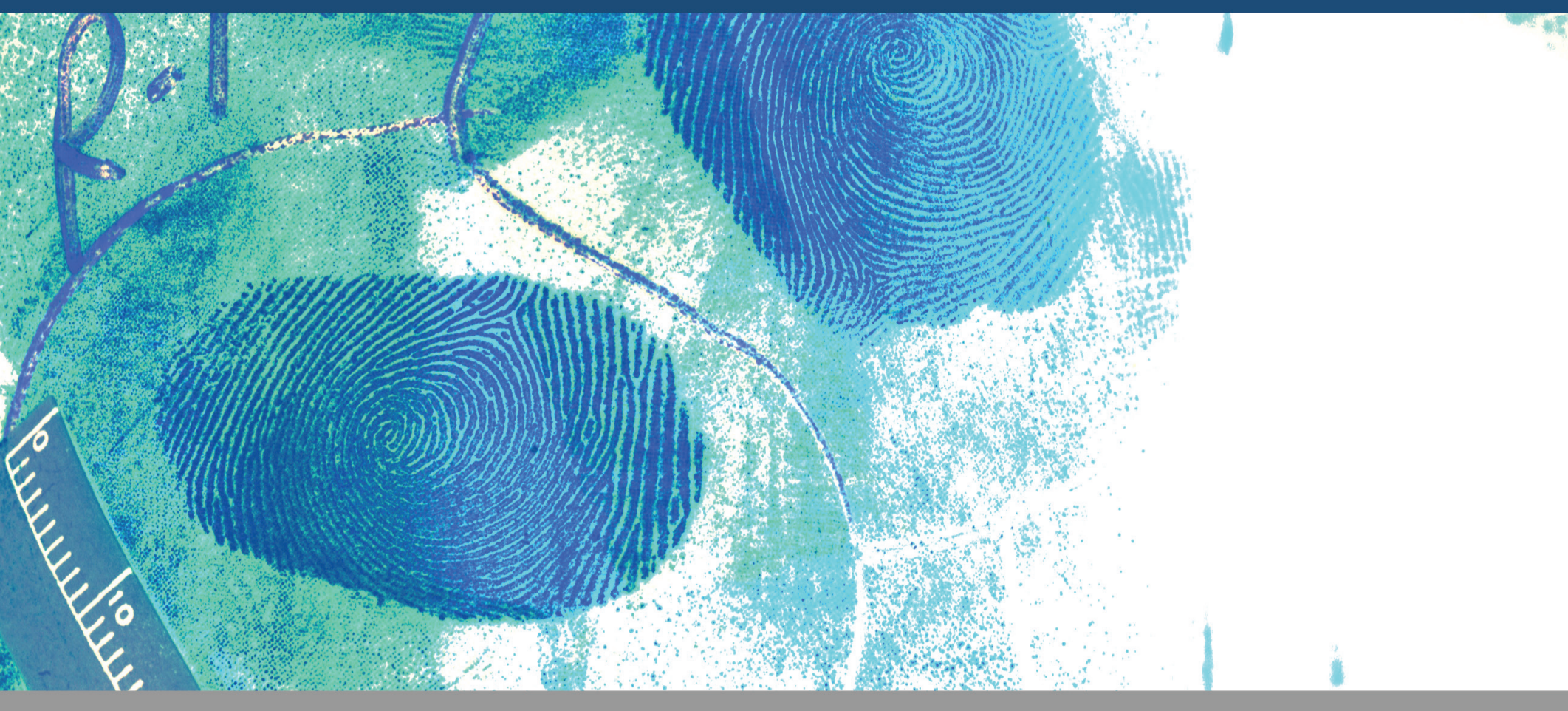

\title{
Latent Print Examination and Human Factors: Improving the Practice through a Systems Approach
}

The Report of the Expert Working Group on Human Factors in Latent Print Analysis

February 2012

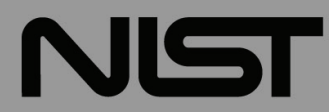

National Institute of Standards and Technology
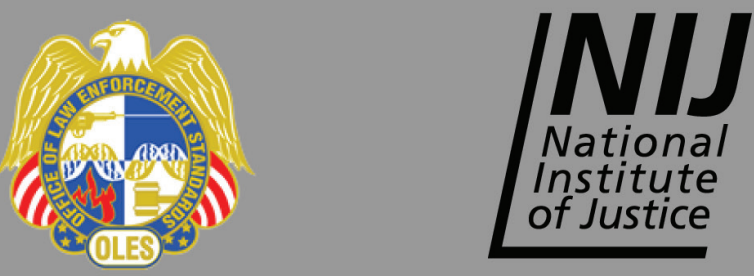


\section{Latent Print Examination and Human Factors: Improving the Practice through a Systems Approach}

\section{The Report of the Expert Working Group on Human Factors in Latent Print Analysis}

\section{In Memoriam}

This report is dedicated to the memory of Danny Greathouse, a valued contributor to this study and a friend who will be missed. 


\section{Latent Print Examination and Human Factors: Improving the Practice}

through a Systems Approach was produced with funding from the U.S. Department of Justice's National Institute of Justice and in collaboration with the Law Enforcement Standards Office in the U.S. Department of Commerce's National Institute of Standards and Technology.

Opinions or points of view expressed in this report are those of the authors and do not necessarily reflect the official position or policies of the U.S. Department of Justice or the U.S. Department of Commerce. Mention of commercial products or services in this report does not imply approval or endorsement by the National Institute of Standards and Technology, nor does it imply that such products or services are necessarily the best available for the purpose.

Suggested citation format: Expert Working Group on Human Factors in Latent Print Analysis. Latent Print Examination and Human Factors: Improving the Practice through a Systems Approach. U.S. Department of Commerce, National Institute of Standards and Technology. 2012. 


\section{Table of Contents}

Introduction vi

Chapter 1: The Latent Print Examination Process and Terminology .......................................1

Chapter 2: Human Factors and Errors .....................................................................................21

Chapter 3: Interpreting Latent Prints ......................................................................................39

Chapter 4: Looking Ahead to Emerging and Improving Technology .....................................77

Chapter 5: Reports and Documentation ..........................................................................90

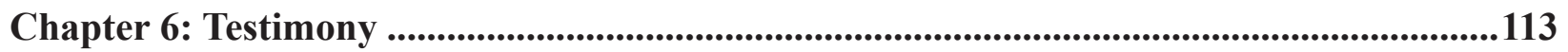

Chapter 7: A Systems Approach to the Work Environment .............................................140

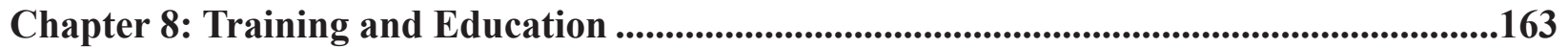

Chapter 9: Human Factors Issues for Management...............................................................172

Chapter 10: Summary of Recommendations ...............................................................................197

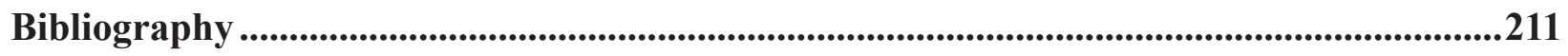




\section{List of Boxes, Figures, and Tables}

\section{Boxes}

Box 1.1: Terminology

Box 1.2: Misconceptions about "Bias" in Science

Box 1.3: Probabilities, Likelihood Ratios, and Individualization

Box 2.1: Daubert v. Merrell Dow Pharmaceuticals, Inc., and "Error Rates"

Box 2.2: How Prevalence, Sensitivity, and Specificity Affect the Posterior Probability of a Correct Positive Identification

Box 2.3: The Zero Numerator Problem

Box 2.4: Selected Results of the Noblis-FBI Experiment

Box 3.1: Studies on the Effect of Biasing Information

Box 7.1: Three Mile Island Accident

Box 9.1: Learning from Others: Benchmarking in Forensic Science

Box 9.2: High-Reliability Organizations

Box 9.3: Accreditation in the European Union

Box 9.4: Certification and Testing for the Practice of Medicine

\section{Figures}

Figure 1.1: The Latent Print Examination Process Map

Figure 1.2: Analysis phase of ACE-V

Figure 1.3: Comparison phase of ACE-V

Figure 1.4: A latent print and exemplar prints

Figure 1.5: Evaluation phase of ACE-V

Figure 1.6: Verification phase of ACE-V

Figure 3.1: Level 1 Detail features

Figure 3.2: Examples of skin distortion on prints of the same finger with arrows indicating location of the same minutiae in different impressions

Figure 4.1: Example of a color replacement filter to remove color from a playing card

Figure 4.2: Example of filters used to adjust color levels and to reverse the colors

Figure 4.3: An example of some minutiae locations in a fingerprint

Figure 7.1: A human factors framework

Figure 7.2: The Hamilton Veale contrast sensitivity test

Figure 7.3: An example of a poorly designed workstation

Figure 7.4: An example of a poorly designed user interface

Figure 9.1: Latent print from an ashtray and an exemplar print

Figure 9.2: Components of a productive system adapted to a latent print unit 


\section{Tables}

Table 1.1: Illustrations of some friction ridge minutiae

Table 1.2: Posterior odds of identity for evidence with a likelihood ratio of 1,000,000 in

populations in which everyone has the same prior odds on being the source of a latent print

Table 2.1: Types of errors and correct conclusions in a binary classification task

Table 2.2: Outcomes of a hypothetical experiment that estimates an examiner's sensitivity and specificity

Table 2.3: Hypothetical data to show probability of identifications in an 80-20 mix

Table 2.4: Hypothetical data to show probability of identifications in a 10-90 mix

Table 2.5: Types of errors and correct conclusions in a binary classification task with the option of not deciding

Table 2.6: Concordancy in judgments of two examiners

Table 2.7: Concordancy with desired outcomes as determined by experts

Table 2.8: Outcomes for pairs judged to be "of value for individualization"

Table 2.9: Accuracy and error rates for exclusions and identifications in pairs judged to be "of value for individualization" and leading to exclusions or identifications

Table 3.1: Distribution for the general patterns on fingerprints from the left and right hands of males $(89,755,960$ fingers)

Table 3.2: Distribution for the general patterns on fingerprints from the right thumb and little fingers of males $(17,951,192$ fingers)

Table 3.3: Examples of statistics on subclassifications

Table 3.4: Relative frequencies for different types of minutiae according to Gupta (1968), Osterburg et al. (1977), and Lin et al. (1982)

Table 3.5: Relative frequencies for different types of minutiae according to Santamaria Beltran (1953), Kingston (1964), Sclove (1979-1980), and Stoney (1985)

Table 6.1: Strength of likelihood ratios in support of evidence

Table 7.1: Recommended eye examinations frequency for adult patients

Table 7.2: Definitions of usability goals and questions that apply specifically to latent print examiners

Table 7.3: Characteristics to consider when designing latent print examiners' work environments

Table 7.4: Characteristics regarding each element of an environment to be considered

Table 9.1: Possible outcomes of the comparison of the latent and exemplar prints in Figure 9.1

Table 9.2: Concordance table listing possible "errors" for conclusions in Table 9.1 


\section{Introduction}

Fingerprints have provided a valuable method of personal identification in forensic science and criminal investigations for more than 100 years. ${ }^{1}$ Fingerprints left at crime scenes generally are latent prints - unintentional reproductions of the arrangement of ridges on the skin made by the transfer of materials (such as amino acids, proteins, polypeptides, and salts) to a surface. Palms and the soles of feet also have friction ridge skin that can leave latent prints. The examination of a latent print consists of a series of steps involving a comparison of the latent print to a known (or exemplar) print. Courts have accepted latent print evidence for the past century. ${ }^{2}$ However, several high-profile cases in the United States and abroad have highlighted the fact that human errors can occur, ${ }^{3}$ and litigation and expressions of concern over the evidentiary reliability of latent print examinations and other forensic identification procedures has increased in the last decade. $^{4}$

"Human factors" issues can arise in any experience- and judgment-based analytical process such as latent print examination. Inadequate training, extraneous knowledge about the suspects in the case or other matters, poor judgment, health problems, limitations of vision, complex technology, and stress are but a few factors that can contribute to errors. A lack of standards or quality control, poor management, insufficient resources, and substandard working conditions constitute other potentially contributing factors.

In addition to reaching correct conclusions in the matching process, latent print examiners are expected to produce records of the examination and, in some cases, to present their conclusions and the reasoning behind them in the courtroom. Human factors issues related to the documentation and communication of an examiner's work and findings therefore merit attention as well.

The study of human factors focuses on the interaction between humans and products, decisions, procedures, workspaces, and the overall environment encountered at work and in daily living. ${ }^{5}$ Human factors analysis can advance our understanding of the nature of errors in complex work settings. Most preventable, adverse events are not just the result of isolated or idiosyncratic behavior but are in part caused by systemic factors. ${ }^{6}$ The forensic science community can benefit from the application of human factors research to enhance quality and productivity in friction

\footnotetext{
${ }^{1}$ Barnes, J. "History." In The Fingerprint Sourcebook. National Institute of Justice, 2011. Cole, S. Suspect Identities: A History of Fingerprinting and Criminal Identification. Harvard University Press, 2001.

${ }^{2}$ Mnookin, J. "Fingerprint Evidence in an Age of DNA Profiling." Brooklyn Law Review, 67 (2001): 13.

${ }^{3}$ E.g., R. v. Smith, 2011 EWCA Crim. 1296; Bertino, A. and P. Bertino. Forensic Science: Fundamentals and Investigations. South-Western Educational Publishing, 2009 (Stephen Cowans case); U.S. Department of Justice, Office of the Inspector General. A Review of the FBI's Handling of the Brandon Mayfield Case (Unclassified and Redacted). U.S. Department of Justice, March 2006; and Sweeney, C. "Lord Advocate to Appear Before Shirley McKie Fingerprint Inquiry." The Times, October 21, 2008.

${ }^{4}$ E.g., Leveson, B. Expert Evidence in Criminal Courts - The Problem, Address to the Forensic Science Society, Kings College, University of London, November 18, 2010. For discussion of specific cases, see Chapter 6. ${ }^{5}$ Sanders, M. and E. McCormick. Human Factors in Engineering and Design, $7^{\text {th }}$ ed. McGraw-Hill Companies, 1993.

${ }^{6}$ National Academy of Sciences, Institute of Medicine, Committee on Quality of Health Care in America. To Err Is Human: Building A Safer Health System. National Academies Press, 1999.
} 
ridge examinations and to reduce the likelihood and consequences of human error at various stages in the interpretation of evidence.

To further this effort, the National Institute of Justice (NIJ) Office of Investigative and Forensic Sciences (OFIS) within the U.S. Department of Justice and the National Institute of Standards and Technology's (NIST's) Law Enforcement Standards Office (OLES) sponsored the work of this expert panel to examine human factors in latent print analysis and to develop recommendations to reduce the risk of error and improve the practice of latent print analysis.

\section{The Expert Working Group on Human Factors in Latent Print Analysis}

The Expert Working Group on Human Factors in Latent Print Analysis was convened in December 2008 and charged with conducting a scientific assessment of the effects of human factors on forensic latent print analysis. A scientific assessment, as defined by the Office of Management and Budget, "is an evaluation of a body of scientific or technical knowledge which typically synthesizes multiple factual inputs, data, models, and assumptions, and/or applies best professional judgment to bridge uncertainties in the available information."

The Working Group was charged with:

- Developing an understanding of the role of human factors and their contributions to errors in latent print analysis through an evidence-based review of literature, case studies, and previous analyses;

- Evaluating approaches to reducing errors in terms of their efficacy, appropriateness in different settings and circumstances, cost, scientific basis, feasibility, institutional barriers to implementation, associated risks, and the quality of evidence supporting the approach;

- Providing guidance to the latent print analysis community on the practical, scientific, and policy outcomes of its work through peer-reviewed publications, presentations at conferences and meetings, and government-sponsored publications;

- Providing guidance to policy-makers and government agencies in promoting a national agenda for error reduction in latent print analysis;

- Attempting to develop credible estimates of the incidence, severity, and costs of errors; and

- Making recommendations for future research.

Working Group members were selected because of their expertise in the forensic sciences or another relevant field and the ability to balance scientific rigor with practical and regulatory constraints. The Working Group consisted of experts from forensic disciplines, statisticians, psychologists, engineers, other scientific experts, legal scholars, and representatives of professional organizations.

The Working Group met 9 times over the course of 2 1/2 years and heard presentations from experts in human factors, vision science, laboratory design, latent print identification, and interpretation in forensic science. Each chapter in this report was developed by a subgroup of the

${ }^{7}$ Office of Management and Budget. Final Information Quality Bulletin for Peer Review. Office of Management and Budget, December 15, 2004. 
Working Group, reviewed by the entire Working Group, edited by a committee within the group, and reviewed again by a set of independent experts. The report was developed through a consensus process in which each Working Group member had an opportunity to influence the recommendations and writing. Despite the diversity of backgrounds and views, the Working Group was able to reach substantial agreement on many important issues, not limited to the formal recommendations. On some matters, however, an irreducible range of opinions remained, and particular chapters indicate those issues.

\section{About the Sponsors}

NIJ is the research, development, and evaluation agency of the U.S. Department of Justice and is dedicated to researching crime control and justice issues. NIJ provides objective, independent, evidence-based knowledge and tools to meet the challenges of crime and justice. The Office of Investigative and Forensic Sciences (OIFS) is the federal government's lead agency for forensic science research and development as well as for the administration of programs that provide direct support to crime laboratories and law enforcement agencies to increase their capacity to process high-volume cases, to provide needed training in new technologies, and to provide support to reduce backlogs. Forensic science program areas include Research and Development in Basic and Applied Forensic Sciences, Coverdell Forensic Science Improvement Grants, DNA Backlog Reduction, Solving Cold Cases with DNA, Postconviction DNA Testing Assistance, National Missing and Unidentified Persons System (NamUs), and Forensic Science Training Development and Delivery.

NIST's mission is to advance measurement science, standards, and technology. It accomplishes these actions for the forensic science community through the OLES Forensic Science Program. The OLES Forensic Science Program directs research efforts to develop performance standards, measurement tools, operating procedures, guidelines, and reports that will advance the field of forensic science. OLES also serves the broader public safety community through the promulgation of standards in the areas of protective systems; detection, enforcement, and inspection technologies; public safety communication; and counterterrorism and response technologies.

\section{Organization of This Report}

Chapter 1 provides an overview of the Analysis, Comparison, Evaluation, and Verification $(\mathrm{ACE}-\mathrm{V})$ process for the examination of latent prints. The chapter also explains certain terminology used throughout the report.

Chapter 2 outlines the general ideas behind human factors and organizational theory. It also addresses the nature of errors in latent print analysis, the reasons for identifying them or measuring their prevalence, and possible ways to estimate accuracy and error rates.

Chapter 3 describes, defines, and clarifies the interpretative stages of latent print comparisons. In addition, it describes the current state of knowledge, based upon published research, and discusses what this research suggests about potential concerns and pitfalls in the interpretive process. 
Chapter 4 surveys new and forthcoming methods, technologies, and techniques. It examines research needs and ways to improve existing technologies for recording and storing exemplars, for utilizing automated searches to locate exemplars for further comparison, and for conducting latent print examinations.

Chapter 5 addresses written reports that summarize and document the results of friction skin impression examinations. Best practices in report writing and documentation increase the likelihood that the evidence is scientifically accurate and will be used appropriately in the administration of criminal justice. The chapter describes the purposes and value of reporting and documenting examinations and makes suggestions regarding the content of these materials. Appendices provide examples of sample reports.

Chapter 6 discusses trial and pretrial communications from the expert to lawyers, judges, and juries. It reviews the more important legal rules and principles that apply to these activities and surveys the types of testimony that might be provided at trial.

Chapter 7 focuses on the conditions under which latent print work is performed that can affect quality in the latent print examination process. It considers issues such as scheduling, lighting, workstations, interruptions, and workplace design.

Chapter 8 reviews the current status of education and training for latent print examiners, requirements and evaluation criteria, and curricula. It makes recommendations for training and educational programs to improve quality and accuracy in latent print analysis and reporting.

Chapter 9 focuses on the role of management in developing and maintaining the system for producing high-quality results. It reviews the components of a quality organization focused on latent print analysis. These include management, personnel, accreditation, certification, proficiency testing, and a systems approach to error identification and mitigation. It recommends actions that managers and the latent print community should take to create or maintain quality latent print units.

Chapter 10 summarizes the most important parts of the preceding chapters. It draws the recommendations from Chapters 3 through 9 into categories that may be helpful for latent print examiners, managers, research funding agencies, researchers, policymakers, and jurists. An appendix lists all formal recommendations in order of their appearance in this report.

Although this report explicitly addresses only the procedures for performing a latent fingerprint examination and communicating the results, much of the analysis and many of the recommendations are applicable to other forensic science disciplines. Issues of cognitive bias, standardization of procedures, documentation of examinations, working conditions, error detection and correction, and accuracy in testimony - among many others - cut across the forensic sciences. By identifying and managing the human factors issues relevant to latent print analysis, the latent print community not only can enhance the quality and accuracy of its contributions to the justice system but also can set an example for other forensic disciplines. The Working Group hopes that this report will assist in this effort. 


\section{Contributors to This Report}

The Expert Working Group on Human Factors in Latent Print Analysis

The Working Group relied upon the contributions of many individuals. The opinions presented over the course of the Working Group's deliberation reflect personal experiences and research. The views in this report do not express the official positions of the institutions with which the members are affiliated.

David H. Kaye, JD, MS, (Editor in Chief) Distinguished Professor and Weiss Family Scholar, Dickinson School of Law and Graduate Faculty Member, Forensic Science Program, Eberly College of Science, Pennsylvania State University

Thomas Busey, PhD, (Editorial Committee) Professor, Department of Psychological and Brain Sciences, Indiana University

Melissa R. Gische, MFS, (Editorial Committee) Physical Scientist/Forensic Examiner, Latent Print Operations Unit, Federal Bureau of Investigation Laboratory

Gerry LaPorte, (Editorial Committee) Forensic Policy Program Manager, National Institute of Justice

Colin Aitken, PhD, Professor of Forensic Statistics, School of Mathematics, University of Edinburgh

Susan Ballou, MS, Law Enforcement Standards Office, National Institute of Standards and Technology

Leonard Butt, Chair, Scientific Working Group on Friction Ridge Analysis, Study and Technology

Christophe Champod, PhD, Professor, Institut de Police Scientifique, École des Sciences Criminelles, Université de Lausanne

David Charlton, PhD, Surrey and Sussex Police Forensic Services, UK

Itiel E. Dror, PhD, University College London and Cognitive Consultants International

Jules Epstein, JD, Associate Professor of Law, Widener University School of Law

Robert J. Garrett, Past President and Chairman of the Board, International Association for Identification

Max M. Houck, PhD, Co-Chair and Principal Analyst, Anser

Edward J. Imwinkelried, JD, Edward L. Barrett, Jr. Professor of Law, Director of Trial Advocacy, University of California, Davis

Ralph Keaton, Executive Director, American Society of Crime Laboratory Directors/Laboratory Accreditation Board

Glenn Langenburg, MS, Forensic Scientist, Minnesota Bureau of Criminal Apprehension

Deborah A. Leben, MS, Lead Fingerprint Specialist, United States Secret Service, Department of Homeland Security

Alice Maceo, Forensic Lab Manager, Las Vegas Metropolitan Police Department Forensic Laboratory

Kenneth F. Martin, Detective Lieutenant (Retired), Crime Scene Services Section, Massachusetts State Police

Jennifer L. Mnookin, JD, PhD, Professor of Law, University of California, Los Angeles 
Cedric Neumann, PhD, Assistant Professor, Department of Statistics and Forensic Science Program, Eberly College of Science, Pennsylvania State University

Joe Polski, Forensics Committee Member, International Association of Chiefs of Police

Maria Antonia Roberts, MS, Research Program Manager, Latent Print Support Unit, Federal Bureau of Investigation Laboratory

Scott A. Shappell, PhD, Professor, Department of Industrial Engineering, Clemson University

Lyle Shaver, Forensic Scientist Supervisor, Virginia Department of Forensic Science

Sargur N. Srihari, PhD, SUNY Distinguished Professor, Department of Computer Science and Engineering, State University of New York at Buffalo

Hal S. Stern, PhD, Professor, Department of Statistics, University of California, Irvine

David Stoney, PhD, Chief Scientist, Stoney Forensic, Inc.

Anjali Swienton, MFS, JD, Director of Outreach, National Clearinghouse for Science, Technology and the Law (NCSTL), Stetson University College of Law

Mary Theofanos, MS, Computer Scientist, Information Access Division, Information Technology Laboratory, National Institute of Standards and Technology

Robert M. Thompson, Program Manager, Forensic Data Systems, Law Enforcement Standards Office, National Institute of Standards and Technology

John Vanderkolk, Laboratory Manager, Indiana State Police Laboratory

Maria Weir, MA, Supervising Forensic Identification Specialist, Los Angeles County Sheriff's Department

Kasey Wertheim, MBA, Co-Chair, President, and CEO, Complete Consultants Worldwide, LLC

Staff

Melissa Taylor, Study Director, Law Enforcement Standards Office, National Institute of Standards and Technology

Mark D. Stolorow, Director, Law Enforcement Standards Office, National Institute of Standards and Technology

Jennifer L. Smither, Lead Editor, Science Applications International Corporation

Kathi E. Hanna, PhD, Consultant Writer and Editor

Shannan Williams, MPP, Program Assistant, Booz Allen Hamilton

\section{Acknowledgements}

The Working Group gratefully acknowledges the following individuals for their contributions to the development of this document.

Bruce Budowle, PhD, Executive Director, Institute of Applied Genetics, Professor, Department of Forensic and Investigative Genetics, University of North Texas Health Science Center

Mike Campbell, Training Coordinator, Ron Smith and Associates, Inc.

Yee-Yin Choong, PhD, Industrial Engineer, Information Technology Laboratory, National Institute of Standards and Technology

Sarah Chu, Innocence Project 
Gislin Dagnelie, PhD, Associate Professor of Ophthalmology, Johns Hopkins University School of Medicine

Susanne M. Furman, PhD, Cognitive Scientist, Information Technology Laboratory, National Institute of Standards and Technology

Danny Greathouse, Former Special Agent, Federal Bureau of Investigation

Marc Green, PhD, Visual Expert Human Factors, Marc Green, Phd and Associates

Austin Hicklin, Noblis

James Johnson, Forensic Consultant, Contracted to United States Secret Service

Philip J. Kellman, PhD, Professor, Department of Psychology, University of California, Los Angeles

Jonathan J. Koehler, PhD, Beatrice Kuhn Professor of Law, Northwestern University School of Law

Kevin Lothridge, Chief Executive Officer, National Forensic Science Technology Center

Tamas Makany, PhD, Cognitive Psychologist

Stephen B. Meagher, Federal Bureau of Investigation Laboratory (Retired)

Ross J. Michaels, PhD, Supervisory Computer Scientist, National Institute of Standards and Technology

Ken Mohr, Senior Forensic Programmer/Planner, Crime Lab Design

Keith Morris, PhD, Associate Professor/Director, Ming Hsieh Distinguished Professor, Forensic and Investigative Science, West Virginia University

Kamran Nouri, Senior Consultant, ABS Consulting

Ron Smith, President, Ron Smith and Associates, Inc.

Brian Stanton, MS, Cognitive Scientist, Information Technology Laboratory, National Institute of Standards and Technology

Lois Tully, Former Deputy Director, Office of Investigative and Forensic Sciences, National Institute of Justice

Lee N. Vanden Heuvel, Manager, Incident Investigation and Root Cause Analysis Services, ABS Consulting

Douglas A. Wiegmann, University of Wisconsin-Madison

David D. Woods, PhD, Professor, The Ohio State University

Reviewers

Individuals chosen for their diverse perspectives and technical expertise reviewed this report in draft form and provided constructive suggestions. These reviewers were not asked to approve or endorse any conclusions or recommendations in the draft report, nor did they review this final version before its release. Responsibility for the final content of this report rests with the members of the Working Group.

John P. Black, Senior Consultant, Ron Smith and Associates, Inc.

Deborah A. Boehm-Davis, PhD, George Mason University

David L. Grieve, Illinois State Police (Retired)

Austin Hicklin, Noblis

Kevin Lothridge, Chief Executive Officer, National Forensic Science Technology Center 
Jennifer S. Mihalovich, F-ABC, Criminalist III, Oakland Police Department Criminalistics Division

Roger C. Park, James Edgar Hervey Distinguished Professor of Law, U.C. Hastings College of Law, San Francisco

Peter Peterson, PhD, Physical Scientist/Forensic Examiner, Federal Bureau of Investigation

Laura Tierney, Certified Latent Print Examiner

Mark L. Weiss, PhD, Division Director, Behavioral and Cognitive Sciences, National Science Foundation

Sandy Zabell, Professor of Mathematics and Statistics, Northwestern University 


\section{Chapter 1: The Latent Print Examination Process and Terminology}

\section{Introduction}

The conventional procedure for associating impressions of friction ridge skin by a latent print examiner involves four phases known as Analysis, Comparison, Evaluation, and Verification (ACE-V). This chapter describes the ACE-V process, notes some of its limitations, identifies areas where human factors should be considered, and defines certain terms used throughout this report.

\section{Box 1.1: Terminology}

$A C E-V$ : An acronym for Analysis, Comparison, Evaluation, and Verification. The ACE-V process is described in section 1.1.

Bias and error: Defined and discussed in section 1.2.

Exemplar or known prints: Prints deliberately collected from an individual, usually fingerprints. Exemplar prints can be collected electronically or by using ink on paper cards. Exemplars may be called ten-prints when impressions of all ten fingers are taken. Exemplar prints collected during criminal arrests normally include one rolled (from one side of the nail to the other) print of each finger pad and a plain or slap impression of each finger.

Focal point: A small region containing distinguishing features within a print.

Forensic service provider: A laboratory or unit that examines physical evidence in criminal matters and provides testimony and reports about the examination findings. In this report, the term is used interchangeably with agency.

Latent print: Unintentional reproduction of the arrangement of ridges on the skin on the underside of the hands or feet made by the transfer of materials from the skin to a surface. This report uses the term print or latent print to denote impressions from all regions of friction ridge skin unless a more specific term such as "fingerprint" or "palm print" is used.

Latent print examination: The study of latent and exemplar prints to help determine the source of the latent print. Because prints come from the friction ridge area of the skin on the hands or feet, latent print analysis is sometimes referred to as friction ridge analysis. As discussed below, "Analysis" and "Comparison" also have specialized meanings in "ACE-V;" therefore, this report generally uses the term "examination" rather than "analysis" or "comparison" when referring to the totality of work of latent print examiners.

Latent print examiner: The individual who conducts the latent print examination, also called latent print analyst. 
Minutiae: Events along a ridge path, including bifurcations (points at which one friction ridge divides into two friction ridges), dots (isolated friction ridge units that have lengths similar their widths), and ridge endings (the abrupt end of ridges), as illustrated in Table 1.1.

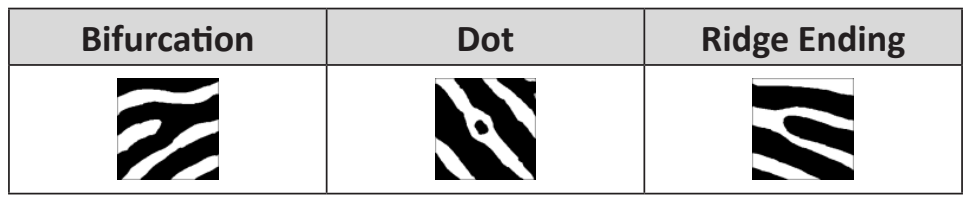

Table 1.1: Illustrations of some friction ridge minutiae ${ }^{8}$

\subsection{The ACE-V Process}

In broad strokes, a latent print examination using the ACE-V process proceeds as follows: Analysis refers to an initial information-gathering phase in which the examiner studies the unknown print to assess the quality and quantity of discriminating detail present. The examiner considers information such as substrate, development method, various levels of ridge detail, and pressure distortions. A separate analysis then occurs with the exemplar print. Comparison is the side-by-side observation of the friction ridge detail in the two prints to determine the agreement or disagreement in the details. In the Evaluation phase, the examiner assesses the agreement or disagreement of the information observed during Analysis and Comparison and forms a conclusion. Verification in some agencies is a review of an examiner's conclusions with knowledge of those conclusions; in other agencies, it is an independent re-examination by a second examiner who does not know the outcome of the first examination.

Figure 1.1, developed by members of the Working Group, describes the steps of the ACE-V process as currently practiced by the latent print examination community. The Latent Print Examination Process Map's purpose is to facilitate discussion about key decision points in the ACE-V process. This chapter briefly describes each step in ACE-V, although the sequence of some of the steps may vary in practice.

\footnotetext{
${ }^{8}$ Images adapted from Champod, C. Reconnaissance Automatique et Analyse Statistique des Minuties sur les Empreintes Digitales. PhD Thesis. Institut de Police Scientifique et de Criminologie, Université de Lausanne, Lausanne, Suisse, 1996.
} 


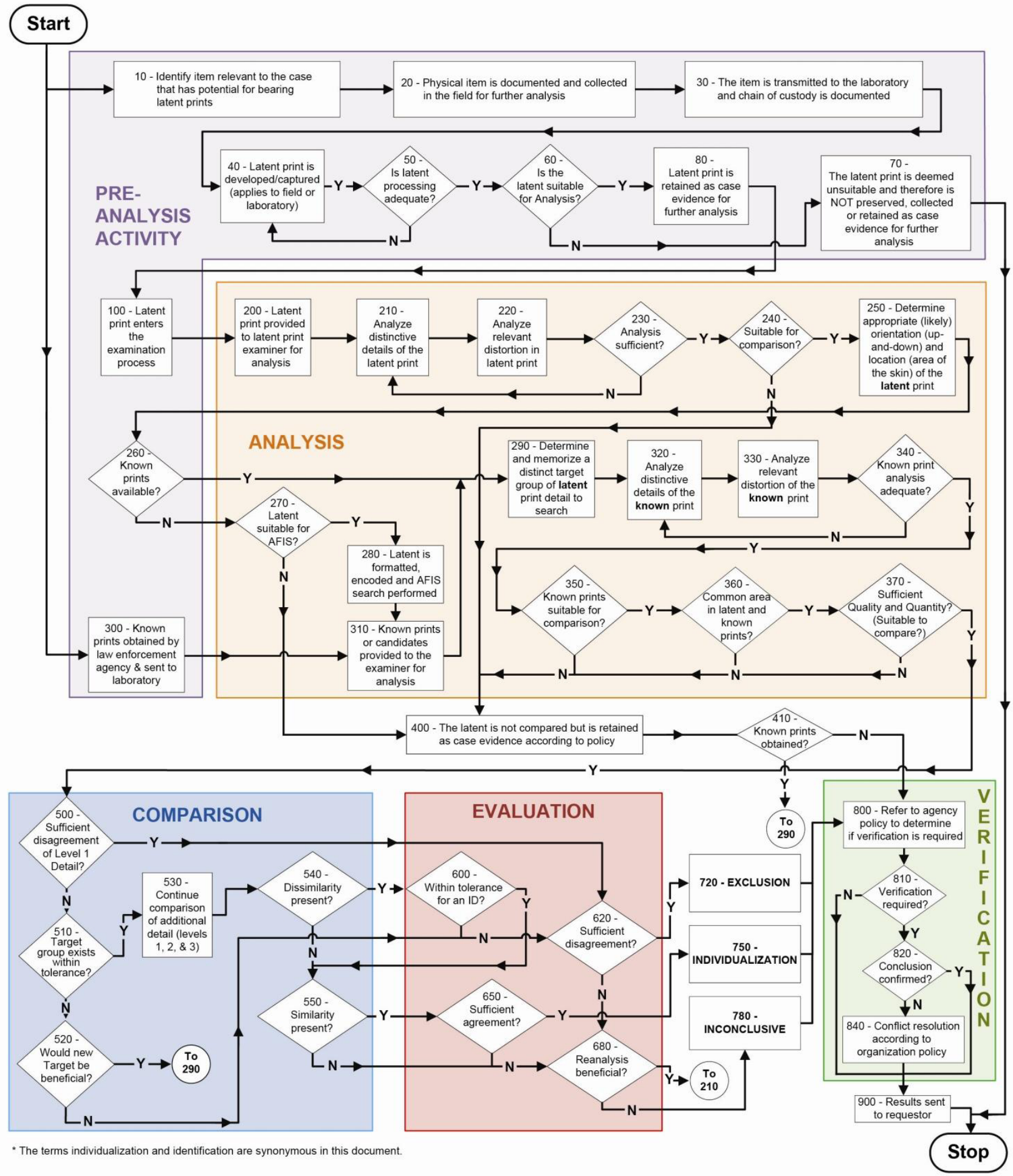

Figure 1.1: The Latent Print Examination Process Map 


\subsubsection{Analysis}

Analysis refers to the initial examination of a friction ridge impression. By inspecting the latent print, the examiner gathers information needed to decide whether it is useful for comparison.

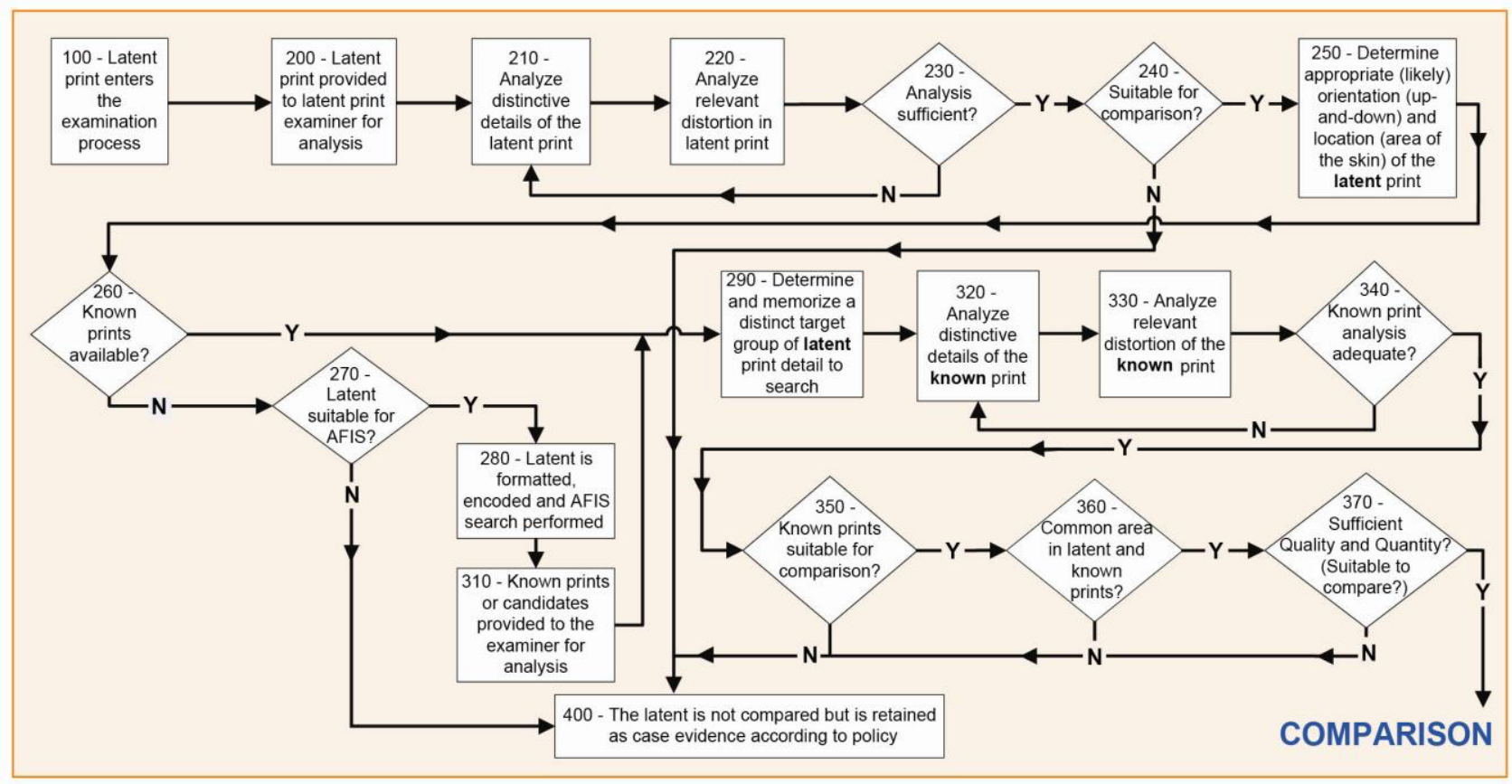

Figure 1.2: Analysis phase of ACE-V

To determine the print's value, the examiner considers three levels of detail in the impression. Level 1 Detail (L1D) is defined as "ridge flow." Ridge flow often translates to a pattern type in a finger or palm, such as a loop, whorl, or arch formation (see Figure 3.1 in Chapter 3); ridge flow also includes other information such as relative curvature. Pattern types are class characteristics shared by many individuals. Level 2 Detail (L2D) is defined as "ridge path."10 L2D includes, but is not limited to, minutiae, such as ridge endings, bifurcations, or dots. Even the absence of minutiae in an area (called an "open field") can be significant and highly discriminating. Level 3 Detail (L3D) is defined as "ridge shapes." Ridge shapes include the edges of ridges (which may appear indented or protruded) and pores (the location of the center of

\footnotetext{
${ }^{9}$ Ashbaugh, D. Quantitative-Qualitative Friction Ridge Analysis: An Introduction to Basic and Advanced Ridgeology. CRC Press, 1999.

${ }^{10} \mathrm{Ibid}$.
} 
the pore, not the size or shape, which can be highly variable within a source). ${ }^{11}$ Chapter 3 , which discusses interpreting information in latent prints, provides additional information on the three levels of detail and their use in the Analysis phase.

After considering the details and the distortion, the examiner judges whether the impression is suitable for a comparison. If the examiner concludes that the print lacks sufficient detail for a comparison, then the examination ends with the determination that the latent print is not suitable for a comparison. Otherwise, the examination moves into the Comparison phase.

\subsubsection{Comparison}

In the Comparison phase, the examiner compares the latent print to one or more exemplar prints. Information gathered in the earlier analysis of the latent print provides a starting point. A comparison of L1D might take only a split second, as when a whorl is present in the latent, but an arch is apparent in the exemplar. If there is no exclusion based upon L1D, then the examiner continues the comparison. If the examiner finds disagreement with respect to the target group that is too extensive to be the result of the distortion noted in the Analysis phase, the examiner will exclude the source of the exemplar as the source of the latent.

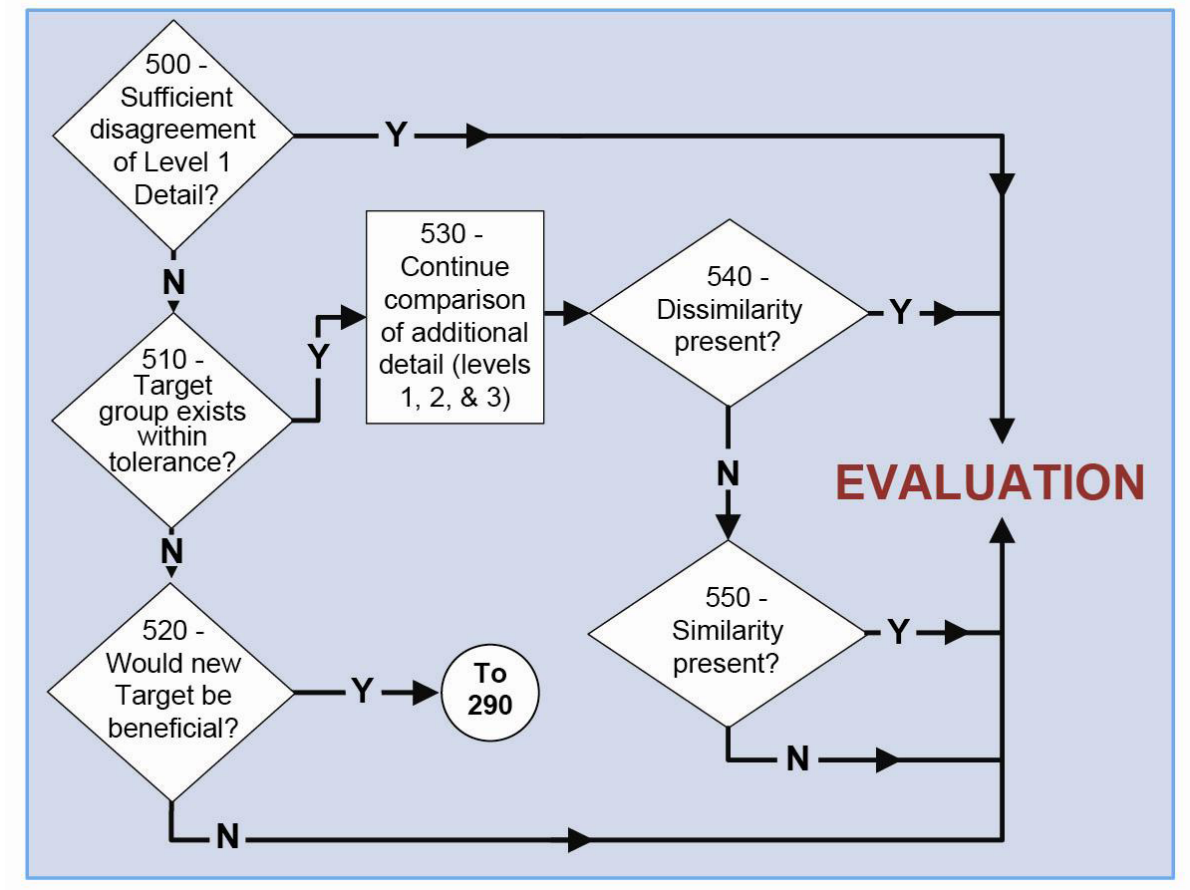

Figure 1.3: Comparison phase of $A C E-V$

\footnotetext{
${ }^{11}$ Roddy, A. and J. Stosz. "Fingerprint Features_-Statistical Analysis and System Performance Estimates," Proceedings of the IEEE, 85, no. 9 (1997): 1390 - 1421; Richmond, S. Do Fingerprint Ridges and Characteristics Within Ridges Change with Pressure?. Australian Federal Police, Forensic Services, 2004.
} 
If the initial target group is not found, alternative target groups may be selected. If the examiner locates a comparable set of L1D features in the known exemplar, the examiner proceeds to a detailed, side-by-side comparison of L2D and possibly L3D. If the examiner concludes that the extent of agreement between the two prints satisfies his or her threshold, then the examiner proceeds to the Evaluation phase.

Figure 1.4 displays a latent print (in the middle) and two very similar exemplar prints from monozygotic (identical) twins. One twin is the source of the latent print. These images were used in an unusually difficult inter-laboratory comparison in 1995.
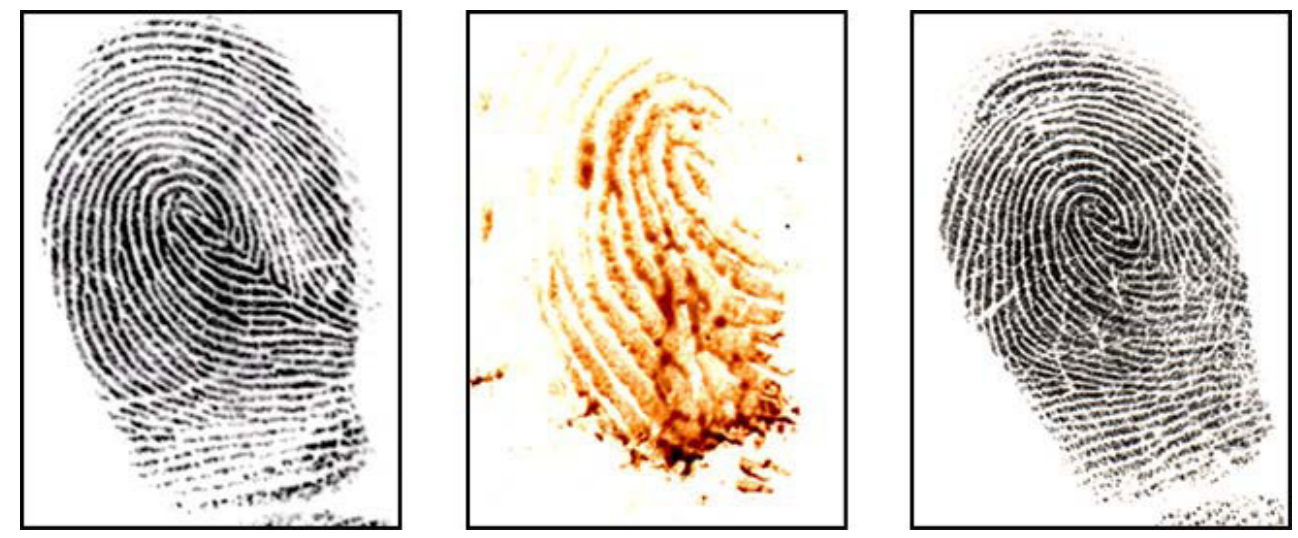

Figure 1.4: A latent print and exemplar prints ${ }^{12}$

\footnotetext{
${ }^{12}$ Images reproduced and adapted with permission of Collaborative Testing Services.
} 


\subsubsection{Evaluation}

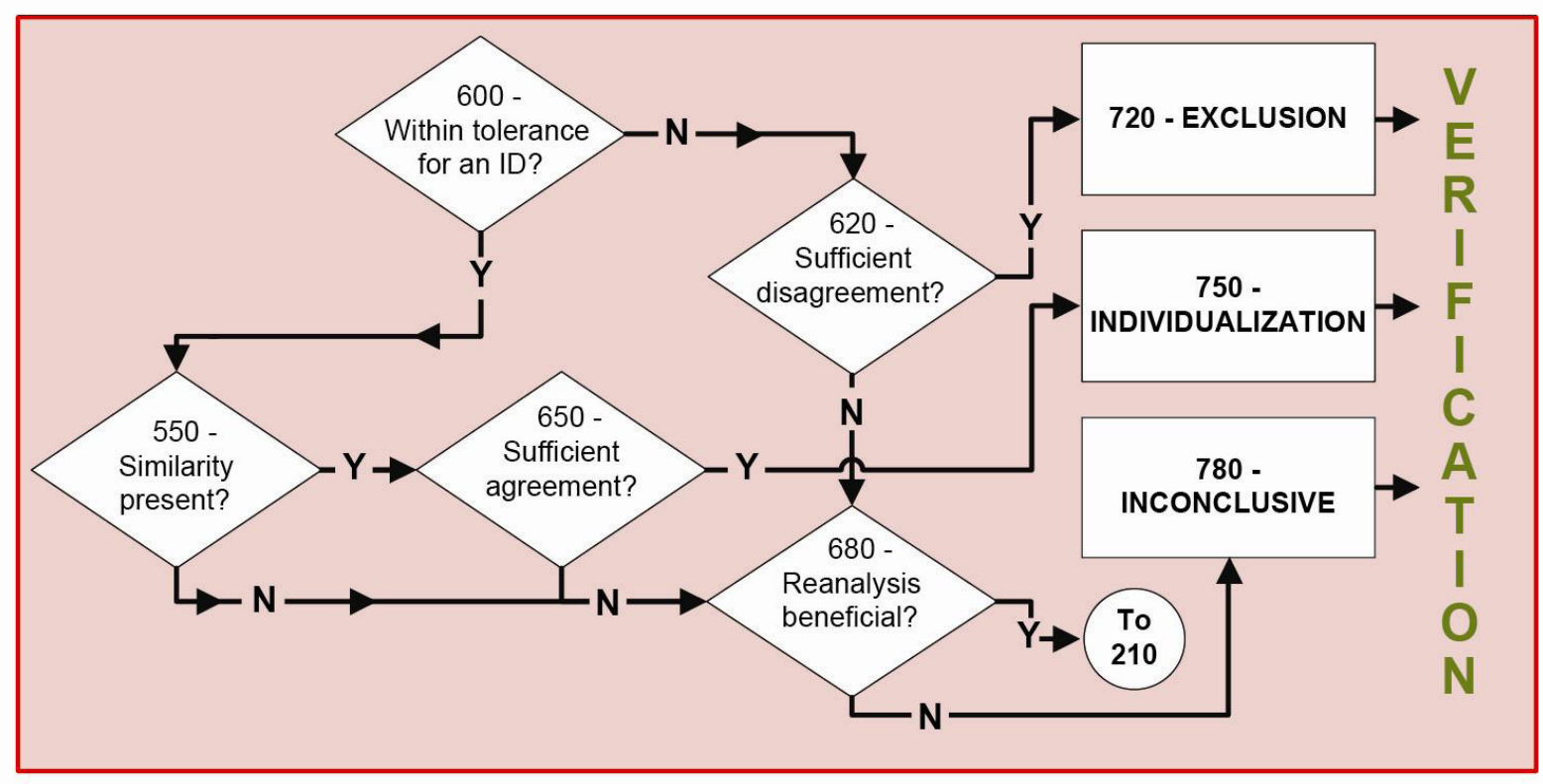

Figure 1.5: Evaluation phase of ACE-V

In the Evaluation phase, the examiner makes the ultimate decision regarding source attribution. Traditionally, three possible findings have been available. First, the examiner can make an "individualization" or "identification." Section 1.2 defines these terms and describes another type of source attribution. Second, the examiner can categorically exclude the latent print by determining that the exemplar print and the latent do not match and cannot share a common source. Finally, the examiner can determine that the information available is inadequate to warrant a conclusion. In that event, the examiner would state that the comparison was "inconclusive" and would provide no additional information about the chances that the two prints share a common source.

The thresholds for these decisions can vary among examiners and among forensic service providers. Some examiners state that they report identification if they find a particular number of relatively rare concurring features, for instance, eight or twelve. Others do not use any fixed numerical standard. Some examiners discount seemingly different details as long as there are enough similarities between the two prints. Other examiners practice the one-dissimilarity rule, excluding a print if a single dissimilarity not attributable to perceptible distortion exists. If the examiner decides that the degree of similarity falls short of satisfying the standard, the examiner can report an inconclusive outcome. If the conclusion is that the degree of similarity satisfies the standard, the examiner reports an identification. 


\subsubsection{Verification}

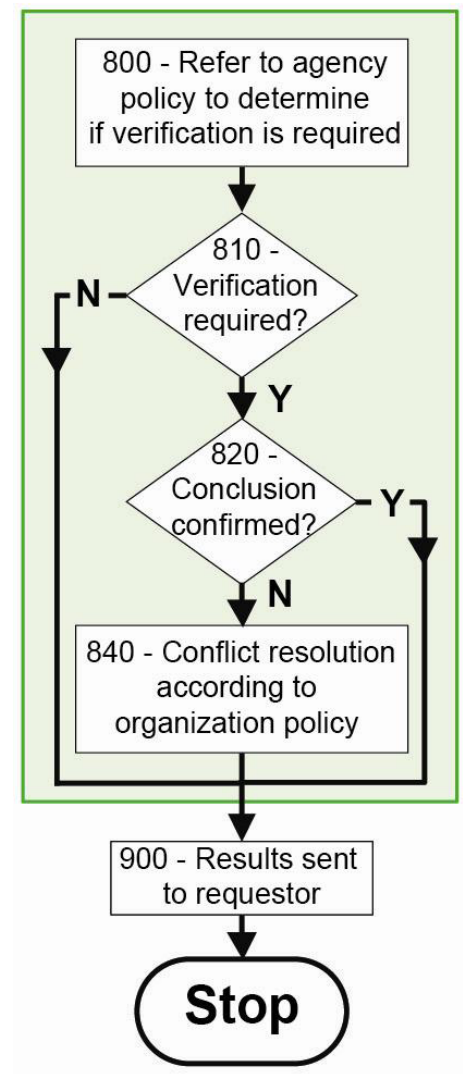

Figure 1.6: Verification phase of ACE-V

In the ACE-V acronym, $V$ stands for Verification. Verification procedures vary among forensic service providers. At one extreme, the verifier, presented with the first examiner's work, assesses the original conclusion. At the other extreme, the verifier, blinded to the initial examination, performs an independent examination.

\subsubsection{Limitations and Concerns about ACE-V}

At every step in the ACE-V process, human factors can affect the outcome. Latent print examiners rely heavily on their training and experience to make the required judgments. Subjectivity is an inextricable part of the process. In the Analysis phase, for example, accurate identification of the characteristics that make prints of value depends on the examiner's knowledge, training, and experience. Likewise, in the Comparison phase, variable factors, such as the elasticity of skin and uneven pressure, mean that there will never be perfect congruence between two prints, even if they originate from the same source. The examiner must resolve the question of whether there is sufficient agreement "within tolerance." As Chapter 3 points out, the examiner at least implicitly relies on a sufficiency threshold to resolve that question, and in setting this threshold, the examiner draws on professional knowledge and experience. There is little research at present that provides objective metrics for determining these tolerances. 
Of course, the mere existence of subjective elements does not make the process unreliable or invalid. Humans can perform many tasks involving subjective judgments quite accurately and consistently. For example, by holding a heavy book and a much lighter one in each hand, most people can subjectively - but correctly - tell which is heavier. Thus, the mere presence of subjectivity is not a valid criticism of the technique, but it does mean that issues related to human factors can be especially salient to the outcome.

Although ACE-V is a systematic process, meaning that the examination proceeds in an orderly and logical fashion, this does not, by itself, demonstrate that the results are accurate and reproducible. In 2009, a committee of the National Research Council (NRC) stated that ACE-V is "a broadly stated framework for conducting friction ridge analyses. However, this framework is not specific enough to qualify as a validated method for this type of analysis. ... Merely following the steps of ACE-V does not imply that one is proceeding in a scientific manner or producing reliable results." 13 Additional study is required to ascertain precisely how well examiners using the process perform under either controlled conditions or in casework, and Chapter 2 describes several possible approaches to developing such information.

Although many in the latent print community describe the ACE-V process as a scientific method (see Chapter 6), the issue is not the label that can or should be attached to the process with respect to human factors. ACE-V is a systematic, skill-based, and widely used process for determining whether two impressions have a common origin. ACE-V designates a logical sequence for a complex process of judgment, but ACE-V itself does not provide substantive guidance about standards to be applied within this sequence. Therefore, even though two examiners might both assert (correctly) that they are using ACE-V, they may be employing different cognitive processes. Those differences create opportunities for human factors to come into play.

\subsection{The Meanings of "Bias" and "Error"}

The issues of bias and error are critical to assessing the role of human factors in latent print analysis. Those terms are described in detail here, and they are referred to throughout this report.

\subsubsection{Bias}

The term "bias" has many meanings. This report discusses the term as it is used in three disciplines. In law, "bias” refers to a witness's partiality toward one party (or against another) as a result of financial, emotional, or other interests or attitudes. The law of evidence does not expect all witnesses to be unbiased. Rather, it relies on the disclosure of the biasing interests or attitudes through cross-examination, a procedure that is effective primarily in exposing gross motivational biases (see Chapter 6).

In statistics, "bias" refers to the extent to which an average statistic departs from the parameter it is estimating or to the extent to which measurements on individual units systematically depart

\footnotetext{
${ }^{13}$ National Academy of Sciences, National Research Council, Committee on Identifying the Needs of the Forensic Science Community. Strengthening Forensic Science in the United States: A Path Forward. National Academies Press, 2009, p. 142.
} 
from the true values. Errors from chance will cancel each other out in the long run; those from bias will not (see Chapter 2).

In psychology, "cognitive bias," which is addressed throughout this report, is a general term for "many observer effects in the human mind, some of which can lead to perceptual distortion, inaccurate judgment, or illogical interpretation."14 Social and cognitive psychologists have identified many sources of cognitive bias, including "information-processing shortcuts (heuristics), motivational factors, and social influence." 15

One type of cognitive bias, called confirmation bias, "is perhaps the best known and most widely accepted notion of inferential error to come out of the literature on human reasoning." 16 Confirmation bias "connotes the seeking or interpreting of evidence in ways that are partial to existing beliefs, expectations, or a hypothesis in hand." 17 It is "a proclivity to search for or interpret additional information to confirm beliefs and to steer clear of information that may disagree with those prior beliefs." "One may be selective in seeking or interpreting evidence that pertains to a belief without being deliberately so, or even necessarily being aware of the selectivity." 19

Observers' expectations have been shown to influence judgment in a broad range of tasks. ${ }^{20}$ Especially when confronted with ambiguous stimuli, people tend to see what they hope or expect to see. Thus, in a clinical trial of an experimental drug, the failure to blind the clinicians evaluating the health of the patients as to whether each patient received the experimental drug or the placebo would be regarded as a serious methodological flaw. This knowledge would create a bias toward a finding of efficacy. Recognition of this cognitive bias in no way impeaches the researcher's integrity and dedication to learning the truth. It simply means that, on average, the measurements the researcher makes could well be shifted systematically from the true values, creating a statistical bias.

As explained in Chapter 3, some information about the origin of a latent print can facilitate accurate results, but other contextual information can produce confirmation bias. ${ }^{21}$ Extraneous information can influence people acting in good faith and attempting to be fair interpreters of the evidence. For example, radiographers may read X-rays differently if they know the patient's

\footnotetext{
${ }^{14}$ Kahneman, D. and A. Tversky. "Subjective Probability: A Judgment of Representativeness." Cognitive

Psychology, 3 (July 1972): 430.

${ }^{15}$ Ibid.

${ }^{16}$ Evans, J. Bias in Human Reasoning: Causes and Consequences. Psychology Press, 1989, p. 41.

${ }^{17}$ Nickerson, R. "Confirmation Bias: A Ubiquitous Phenomenon in Many Guises." Review of General Psychology, 2 (1998): 175.

${ }^{18}$ Budowle, B., M. Bottrell, S. Bunch, et al. "A Perspective on Errors, Bias, and Interpretation in the Forensic

Sciences and Direction for Continuing Advancement." Journal of Forensic Sciences, 54 (July 2009): 803.

${ }^{19}$ Nickerson, op. cit., p. 176.

${ }^{20}$ See Miller, L. "Procedural Bias in Forensic Science Examinations of Human Hair." Law and Human Behavior, 11, no. 2 (June 1987): 157; Nickerson, op cit.; and Risinger, D., M. Saks, W. Thompson, et al. "The Daubert/Kumho Implications of Observer Effects in Forensic Science: Hidden Problems of Expectation and Suggestion." California Law Review, 90, no. 1 (January 2002).

${ }^{21}$ See, e.g., Saks, M., D. Risinger, R. Rosenthal, et al. "Context Effects in Forensic Science: A Review and Application of the Science of Science to Crime Laboratory Practice in the United States." Science and Justice, 43, no. 2 (April - June 2003): 77.
} 
clinical history; ${ }^{27}$ doctors may assess a patient differently in a drug trial if they know whether the patient is receiving the experimental drug or a placebo, ${ }^{28}$ and a consumer may express different taste preferences in response to food labels or knowledge of the ingredients included. 29 "Contextual bias" occurs when "the forensic scientist uses other evidence to believe that the specific evidence being analyzed is related to a particular reference sample(s)",30 and when the contextual information prompts a biased selection or weighting of the features in the samples.

In short, "bias" can be a loaded term, as when people speak of personal bias, racial bias, gender

\section{Box 1.2: Misconceptions about "Bias" in Science}

Cognitive scientists and psychologists have observed cognitive bias in hundreds of scientific studies across dozens of domains. ${ }^{22}$ Research has also demonstrated its existence in latent print examination such as when one examiner, presented with consistent evidence in different biasing contexts, has been shown to reach different conclusions. ${ }^{23}$ Much of the work in latent print examination depends on judgment and decision-making. In many scientific fields, such judgments are recognized as subjective and vulnerable to contextual biases.

Within the forensic science community, some people still lack an understanding of what bias is and how best to address it. ${ }^{24}$ Often, cognitive bias is treated as an ethical issue or as an issue that will resolve once someone is aware of the problem. However, the cognitive process used when gaining experience (e.g., using schemas, chunking information, automaticity, and more reliance on top-down information) in itself opens the practitioner to vulnerabilities, including bias, tunnel vision, lack of flexibility, and selective attention..$^{25}$ Cognitive bias results from computational trade-offs carried out in the brain and is not a conscious act or an act that can be avoided at will. ${ }^{26}$

\footnotetext{
${ }^{22}$ E.g., Nickerson, op cit.
}

${ }^{23}$ For a review, see Dror, I. and R. Rosenthal. "Meta-Analytically Quantifying the Reliability and Biasability of Forensic Experts.” Journal of Forensic Sciences, 53 (July 2008): 900. See also Dror, I. and S. Cole. "The Vision in 'Blind' Justice: Expert Perception, Judgment, and Visual Cognition in Forensic Pattern Recognition." Psychonomic Bulletin \& Review, 17 (2010): 161.

${ }^{24}$ Pronin, E. "Perception and Misperception of Bias in Human Judgment." Trends in Cognitive Science, 11 (January 2007): 37.

${ }^{25}$ Dror, I. "The Paradox of Human Expertise: Why Experts Can Get It Wrong." In The Paradoxical Brain, edited by N. Kapur. Cambridge University Press, 2011; and Busey, T. and I. Dror. "Special Abilities and Vulnerabilities in Forensic Expertise." In The Fingerprint Sourcebook. National Institute of Justice, 2011.

${ }^{26}$ E.g., McClelland, J. and D. Rumelhart. "An Interactive Activation Model of Context Effects in Letter Perception: Part 1, An Account of Basic Findings.” Psychological Review, 88 (September 1981): 375; and Wilson, T. and N. Brekke. "Mental Contamination and Mental Correction: Unwanted Influences on Judgments and Evaluations." Psychological Bulletin, 116 (July 1994): 117.

${ }^{27}$ See, e.g., Tape, T. and R. Panzer. "Echocardiography, Endocarditis, and Clinical Information Bias." Journal of General Internal Medicine, 1, no.3 (1986): 300; Schreiber, M. "The Clinical History as a Factor in Roentgenogram Interpretation." The Journal of the American Medical Association, 185 (1963): 137; Doubilet, P. and P. Herman. "Interpretation of Radiographs: Effect of Clinical History." American Journal of Roentgenology, 137, no. 5 (1981): 1055; and Potchen, E., J. Gard, P. Lazar, et al. "The Effect of Clinical History Data on Chest Film Interpretation: Direction or Distraction?” Investigative Radiology, 14 (1979): 404.

${ }^{28}$ Levine, R. Ethics and Regulation of Clinical Research. 2nd ed. Yale University Press, 1986; and Shultz, K. and ${ }^{\mathrm{D}}$. Grimes. "Blinding in Randomized Trials: Hiding Who Got What." Lancet, 359 (February 2002): 696.

${ }^{29}$ Allison, R. and K. Uhl. "Influence of Beer Brand Identification on Taste Perception." Journal of Marketing Research, 1 (1964): 36; Nevid, J. "Effects of Brand Labeling on Ratings of Product Quality. Perceptual and Motor Skills, 53 (1981): 407; Palmer, S. "The Effects of Contextual Scenes on the Identification of Objects.” Memory and Cognition, 3 (1975): 519; and Lee, L., S. Frederick, and D. Ariely. "Try It, You'll Like It: The Influence of Expectation, Consumption, and Revelation on Preferences for Beer.” Psychological Science, 17 (2006): 1054.

${ }^{30}$ Budowle, Bottrell, Bunch, et al., op. cit., p. 803. 
bias, cultural bias, media bias, political bias, and so on. However, this is not the type of bias with which this report is concerned. Although there have been occasional cases of conscious biasindeed, outright fraud - in forensic science ${ }^{31}$ (as in all sciences and professions), cognitive bias is far more subtle and usually unknown to the observer. Just how often such bias produces incorrect findings is difficult to say, ${ }^{32}$ but "the possibility of biases influencing the decision-making process of examiners" cannot be dismissed. ${ }^{33}$

The issue of cognitive bias is therefore one important topic in the study of human factors in friction ridge impression examination. Research on expectancy effects in latent print identification is limited. ${ }^{34}$ However, being consistent with widely accepted psychological phenomena, this research has prompted proposals for blinding forensic examiners to the origin of samples being compared with each other ${ }^{35}$ and for using multiple exemplars in comparisons. ${ }^{36}$ Likewise, blind verification shields the verifying examiner from contextual bias that might otherwise affect the outcome in difficult cases. The Noblis-FBI experiment, discussed in Chapter 2 , indicated "that blind verification of exclusions could greatly reduce false negative errors." 37 Taking the human factors perspective, an agency might wish to adopt one or more of these systemic changes rather than simply warning examiners to do their best not to be influenced by potentially biasing information. ${ }^{38}$

\subsubsection{Error}

Like "bias," the word "error" has a multitude of possible meanings. This report addresses the relationship between human factors and the two concepts of error outlined in Chapter 2procedural error and outcome error. Procedural error refers to departures from a prescribed procedure. The failure of a technician to calibrate an instrument for measuring breath alcohol concentration as frequently as regulations prescribe, for example, is a procedural error. It

\footnotetext{
${ }^{31}$ E.g., Giannelli, P. "Independent Crime Laboratories: The Problem of Motivational and Cognitive Bias.” Utah Law Review, 247 (2010): 251.

${ }^{32}$ Langenburg, G., C. Champod, and P. Wertheim. "Testing for Potential Contextual Bias Effects During the Verification Stage of the ACE-V Methodology When Conducting Fingerprint Comparisons." Journal of Forensic Sciences, 54 (May 2009): 571 - 82 (concluding that blind verification may be most useful for exclusions).

${ }^{33}$ Scientific Working Group on Friction Ridge Analysis, Study and Technology. Strengthening Forensic Science in the United States: A Path Forward, Position Statement. Scientific Working Group on Friction Ridge Analysis, Study and Technology, 2009; U.S. Department of Justice, Office of the Inspector General. A Review of the FBI's Handling of the Brandon Mayfield Case (Unclassified and Redacted). U.S. Department of Justice, 2006; Stacey, R. "A Report on the Erroneous Fingerprint Individualization in the Madrid Train Bombing Case." Journal of Forensic Identification, 54, no. 6 (2004): 706; and Moses, K. “Anatomy of Error." Presented at International Association for Identification 89th International Educational Conference, St. Louis, Missouri. August 2004.

${ }^{34}$ Dror and Cole, op. cit., p. 161; Dror, I., D. Charlton, and A. Peron. "Contextual Information Renders Experts Vulnerable to Making Erroneous Identifications.” Forensic Science International, 156, no. 1 (2006): 74 - 78; Dror, I. and D. Charlton. "Why Experts Make Errors." Journal of Forensic Identification, 56, no. 4 (2006): 600 - 16; Dror and Rosenthal, op. cit.; and Langenburg, Champod, and Wertheim, op. cit.

${ }^{35}$ Krane, D., S. Ford, J. Gilder, et al. "Sequential Unmasking: A Means of Minimizing Observer Effects in Forensic DNA Interpretation.” Journal of Forensic Sciences, 53 (2008): 1006.

${ }^{36}$ NAS, NRC, Committee on Identifying the Needs of the Forensic Science Community, op. cit., p. 123.

${ }^{37}$ Ulery, B., A. Hicklin, J. Buscaglia, et al. "Accuracy and Reliability of Forensic Latent Fingerprint Decisions." Proceedings of the NAS. 2011.

${ }^{38}$ Following the misidentification of Brandon Mayfield as a Madrid train bomber, for instance, the Federal Bureau of Investigation (FBI) implemented blind verification in some cases. U.S. Department of Justice, Office of the Inspector General, op. cit.; Stacey, op. cit.; and Moses, op. cit.
} 
increases the risk of an error in the actual measurements, ${ }^{39}$ but this risk might not be realized; the instrument could remain correctly calibrated during the period in question and thus be no more likely to err than it would have if the technician had followed the proper calibration schedule.

Whether or not the measuring instrument is calibrated in the prescribed manner, it is unlikely to give perfectly accurate results. Some measurements will be above the true value, some below it. For nominal variables (involving categories, such as the ABO types of blood), measurement error usually is called misclassification or classification error. ${ }^{40}$

When human beings make comparisons, they also engage in a form of measurement. Visually determining that one source of light is bright and another dim, or that one object is large and another small, are examples of classifications by a human system for sensory measurement. Likewise, the human examiner following the ACE-V process acts as a measuring instrument, and the examination of two friction ridge skin impressions is a form of categorical measurement or decision-making. An examiner who reaches a conclusion that two impressions of friction ridge skin do or do not match is making a binary classification, and it might be a misclassification or outcome error. If the exclusion or inclusion corresponds to the true state of affairs, there is no such outcome error. A judgment of an exclusion when the two impressions come from the same individual's skin is a false exclusion (a false negative). A declaration of a match when the two impressions come from different individuals is a false inclusion (a false positive). Chapter 3 develops these ideas further.

\subsubsection{Identification: Inclusions and Exclusions}

Forensic scientists are sensitive to the breadth of the word "identification." It can mean placing an item in a large class, as in "I identified this automobile as a red Buick." 41 It also can mean associating a trace with a single possible source, as when a latent print examiner reports, "I identified the latent print as having been made by the right ring finger of the defendant." 42 Examinations of features for the purpose of identification can lead to inclusions (for example, the suspect's red Buick is included in the set of automobiles that might have been used by the robbers) or exclusions (for example, the suspect's green Lincoln is excluded as the one used in the robbery). As these examples indicate, an inclusion increases the probability that a trace originated from a particular source within that set, and an exclusion decreases this probability to essentially zero. In short, in forensic science and law generally, identifying information makes an association between a source and a trace either more probable or less probable. If the shifted probability is large enough, it can justify a source attribution.

\footnotetext{
${ }^{39}$ See Flaherty, M. “400 Drunken-Driving Convictions In D.C. Based On Flawed Test, Official Says.” The Washington Post, June 10, 2010.

${ }^{40}$ Rothman, K., S. Greenland, and T. Lash. Modern Epidemiology. 3d ed. Lippincott, Williams, \& Wilkins, 2008 , p. 138.

${ }^{41}$ Thornton, J. and J. Peterson. "The General Assumptions and Rationale of Forensic Identification." Modern Scientific Evidence: The Law and Science of Expert Testimony. West Publishing Company, 1997.

${ }^{42}$ Ibid.
}

13 Latent Print Examination and Human Factors: Improving the Practice through a Systems Approach

The Report of the Expert Working Group on Human Factors in Latent Print Analysis 
Latent print examiners often use the word "identification" more narrowly, to denote the finding of an association at the conclusion of the process known as ACE-V. ${ }^{43}$ In this more specialized vocabulary, an identification is sometimes also referred to as an individualization. ${ }^{44}$ In general, however, forensic "identification" encompasses observations that could narrow the set of possible sources of a trace and thus change the probability that the trace originated from a particular source within that set, even when there is more than one remaining possible source. Whether and when it is appropriate to conclude that the identification procedure establishes that the set of possible sources consists of one and only one individual is discussed below in connection with the term "individualization."

\subsubsection{Individualization}

As the discriminating power of the latent print features used to make an identification increases, so does the probability that the matching individual is the source. When every possible source has different features, and when these differences are detectable, a match in the feature set can establish that a specific individual is the source. In this limiting case, when the features in the samples have been accurately ascertained, compared, and found to match, the probability that the individual in question is the source is one. The identification then has such specificity that it has become an "individualization."

The classical theory of latent print identification is that individualization is possible regardless of how many people could be considered as possible sources. This theory holds that in the population of the entire world (both in the distant past and the far future): (1) every finger has a unique pattern of ridges and other features that is maintained throughout a person's lifetime, ${ }^{45}$ and (2) a well-trained examiner can ascertain when any impression from a finger displays enough features to distinguish it from every sufficiently complete and clear impression of every other finger. This is a theory of "universal individualization" based on a premise of "general uniqueness." 46

In practice, a universal individualization means that the examiner is confident that if impressions from everyone else who ever lived and ever will live could be compared with the latent print, then not one would match. ${ }^{47}$ The individualizing examiner effectively sets the size of the

\footnotetext{
${ }^{43}$ E.g., Scientific Working Group on Friction Ridge Analysis, Study and Technology. Standards for Conclusions. Version 1.0. Scientific Working Group on Friction Ridge Analysis, Study and Technology, September 2003.

${ }^{44}$ E.g., Scientific Working Group on Friction Ridge Analysis, Study and Technology. Standard Terminology of Friction Ridge Examination (To Replace: Glossary, Version 2.0). Version 3. Scientific Working Group on Friction Ridge Analysis, Study and Technology, February 11, 2011. This terminology defines "identification" as "individualization" and notes that in other forensic disciplines "this term denotes the similarity of class characteristics."

${ }^{45}$ E.g., Peterson, P., C. Dreyfus, M. Gische, et al. "Latent Prints: A Perspective on the State of the Science." Forensic Science Communications, 11, no. 4 (October 2009); Stoney, D. "Measurement of Fingerprint Individuality." In Advances in Fingerprint Technology, edited by Henry C. Lee and R.E. Gaensslen, 327-87. 2nd ed. CRC Press, 2001.

${ }^{46}$ Kaye, D., D. Bernstein, and J. Mnookin. The New Wigmore, A Treatise on Evidence: Expert Evidence. 2nd ed. Aspen Publishers, 2011. "General uniqueness" means that every element of a set is distinguishable from every other element. "Special uniqueness" means that a particular element is distinguishable from all others even if not all of the remaining elements are each distinguishable. Kaye, D. "Identification, Individualization and Uniqueness: What's the Difference?" Law, Probability \& Risk, 8 (2009): 85.

${ }^{47}$ Stoney, 2001, op. cit., p. 332.
} 
population of possible sources to its maximum (the entire population of the earth) regardless of the specific circumstances of the case. The individualization process moves from this maximum initial population to a decision. ${ }^{48}$ At the end of the examination process, the quantity of features observed in agreement between two objects (without significant discrepancies) is perceived as so impressive that the examiner has ruled out the possibility of a coincidental match, whatever initial population of sources was involved. This has been described as a "leap of faith." 49 It means that the identification of the source is to the exclusion of all other sources.

In recent years, the classical theory of universal individualization has come under attack in court and in the academic literature (see Chapter 6). Some critics of individualization dismiss the first premise of global, general uniqueness as unscientific and metaphysical. ${ }^{50}$ In principle, however, belief in uniqueness is compatible with scientific and statistical reasoning. ${ }^{51}$ Nonetheless, the fact that the surfaces of every individual's fingers are unique (at a sufficient level of detail) is not, in itself, a persuasive argument for universal individualization. ${ }^{52} \mathrm{In}$ a sense, the uniqueness argument proves too much, for just as every three-dimensional surface of every finger presumably is unique, so is every latent print left on two-dimensional surfaces, even those from the same skin. ${ }^{53}$ Consequently,

uniqueness does not guarantee that prints from two different people are always sufficiently different that they cannot be confused, or that two impressions made by the same finger will also be sufficiently similar to be discerned as coming from the same source. The impression left by a given finger will differ every time, because of inevitable variations in pressure, which change the degree of contact between each part of the ridge structure and the impression medium. ${ }^{54}$

This takes us to the second proposition underlying the claim of universal individualization. How can we know that a skilled examiner can consistently discriminate between pairs of prints that come from the same source and pairs that come from different sources when there is substantial variation in both types of pairs? The question of the variability of multiple impressions from the same source versus inter-source variability is not special to latent print analysis. It is fundamental to the recognition of individual sources across the forensic sciences and to signaldetection problems in engineering and psychology. In 1979, it prompted an NRC committee to

\footnotetext{
${ }^{48}$ Biedermann, A., S. Bozza, and F. Taroni. "Decision Theoretic Properties of Forensic Identification: Underlying Logic and Argumentative Implications." Forensic Science International, 177 (2008): 120.

${ }^{49}$ Stoney, D. "What Made Us Ever Think We Could Individualize Using Statistics?" Journal of the Forensic Science Society, 31 (1991): 197.

${ }^{50}$ Saks, M. and J. Koehler. "The Individualization Fallacy in Forensic Science Evidence." Vanderbilt Law Review, 61 (2008): 199.

${ }^{51}$ Kaye, D. "Probability, Individualization, and Uniqueness in Forensic Science Evidence: Listening to the Academies." Brooklyn Law Review, 75 (Summer 2010): 1163; National Academy of Sciences, National Research Council, Committee on DNA Forensic Science: An Update. The Evaluation of Forensic DNA Evidence. National Academies Press, 1996, p. 161.

${ }^{52}$ Stoney, 1991, op. cit., p. 331.

${ }^{53}$ Vanderkolk, J. Forensic Comparative Science: Qualitative Quantitative Source Determination of Unique Impressions, Images, and Objects. Academic Press, 2009, p. 195; and Kaye, D. "Questioning a Courtroom Proof of the Uniqueness of Fingerprints." International Statistical Review, 71 (2003): 521. Compare Cole, S., M. Welling, R. Dioso-Villa, et al. "Beyond the Individuality of Fingerprints: A Measure of Simulated Computer Latent Print Source Attribution Accuracy." Law, Probability \& Risk, 7 (2008): 165.

${ }^{54}$ NAS, NRC, Committee on Identifying the Needs of the Forensic Science Community, op. cit. p. 144.
} 
question the ability of spectrographic analysis to provide voiceprints that can be associated with one and only one individual. ${ }^{55}$ Similarly, the 2009 NRC report asks for "studies [that] would accumulate data about the intra-individual variability (e.g., how much one finger's impressions vary from impression to impression, or how much one toolmark or signature varies from instance to instance) and the inter-individual variability (e.g., how much the impressions of many fingerprints vary across a population and in what ways)" to validate the ACE-V process and "to attach confidence limits to individualization determinations." 56 To be sure, "examiners are trained to recognize the factors that may affect the translation of information [from three dimensions to two];" 57 yet the fact remains that "none of these variabilities - of features across a population of fingers or of repeated impressions left by the same finger-has been characterized, quantified, or compared." 58

Despite such misgivings, many fingerprint experts believe that every latent print (of sufficient quality, as judged by a human examiner with no predefined, articulable standard for ascertaining sufficiency) from a single finger has a degree of similarity to a rolled or plain print that is measurably greater than its similarity to every other finger on Earth. To appreciate just how ambitious this claim is, suppose that the chance of two latent fingerprints (from two different individuals) appearing so similar as to be declared a match is a mere one in one trillion. Because the Earth's population is approximately seven billion people, it might seem that no pair of fingers from different individuals now living would match. After all, one in one trillion is two orders of magnitude smaller than one in seven billion. However, seven billion individuals give rise to immensely more pairs of prints that, by coincidence, could match. All told, there are nearly 25 $\times 10^{18}$ distinct pairs of individuals to be considered. Individual 1 can be paired with Individuals 2 through 7,000,000,000 (for seven billion comparisons). Individual 2 can be paired not only with Individual 1 but also with Individuals 3 through 7,000,000,000 (for another seven billion comparisons).Statistically, the expected number of indistinguishable pairs from different sources is the number of possible comparisons times the probability of a match for each comparison. This expected number of matching pairs is $25 \times 1018 \times 10^{-12}=25$ million.

Of course, this particular number is hypothetical. Perhaps some prints are so distinctive that the probability of confusing them with any print from any other finger is less than one in one trillion. The computation could be refined to handle heterogeneity in random-match probabilities, but the purpose here is not to estimate any particular number of matching pairs that might exist.

\footnotetext{
${ }^{55}$ National Academy of Sciences, National Research Council, Committee on Evaluation of Sound Spectrograms. On the Theory and Practice of Voice Identification. National Academies Press, 1979, p. 4.

${ }^{56}$ NAS, NRC, Committee on Identifying the Needs of the Forensic Science Community, op. cit., p. 184.

${ }^{57}$ Peterson, Dreyfus, Gische, et al., op. cit. Compare NAS, NRC, Committee on Identifying the Needs of the Forensic Science Community, op. cit., p. 145.

${ }^{58}$ NAS, NRC, Committee on Identifying the Needs of the Forensic Science Community, op. cit., p. 144. SWGFAST, 2009, op. cit. complains that "a significant body of constructive scientific research ... has not been adequately reported by the NAS committee." Yet, the statement cites no such "significant body" of research that fills the specific gap in the proof of the individualization hypothesis noted by the NRC report. Peterson, Dreyfus, Gische, et al., op . cit. refer to an "FBI Laboratory manuscript in preparation" that finds that "controlled recordings from the friction ridge skin have shown that the information contained in friction ridge impressions does translate reliably as a true and accurate representation of what appears on the friction ridge skin."
} 
It is merely to underscore the nearly inconceivable number of pairs of prints that have to be considered to establish the premise of universal individualization. ${ }^{59}$

Given these difficulties, there has been some movement toward using the term "individualization" to mean any definitive identification of a single individual as the source of a latent print, even one that falls short of universality. The Scientific Working Group on Friction Ridge Analysis, Study and Technology has eliminated the global "exclusion of all others" language in its glossary ${ }^{60}$ and plans to do the same in its standards. ${ }^{61} \mathrm{~A}$ draft guideline defines individualization as "the decision by an examiner that there are sufficient features in agreement to conclude that two areas of friction ridge impressions originated from the same source." ${ }^{62}$ As to how this decision is made, the next sentence adds, "Individualization of an impression to one source is the decision that the likelihood the impression was made by another (different) source is so remote that it is considered as a practical impossibility." 63

To some extent, however, the new definition begs the question of how the examiner knows that the probability that the "impression was made by another (different) source" is practically zero. Is the rationale that individualization is still universal, applying as a "practical" matter to astronomical numbers of possible pairs? If the "practical" judgment remains based upon the classical theory of universal individualization, then an individualization is still a statement that the examiner has excluded everyone else in the world as the source of the latent print.

Perhaps the new form of "individualization" is simply a source attribution based upon an examiner's training and experience with two sets of paired latent and exemplars in which the origin of the prints is known. One set consists of all pairs of latent and exemplar prints known to the examiner to have come from the same fingers (mates). The other set consists of all pairs known to the examiner to have come from different fingers (nonmates). An examiner might conclude that the prints being compared are more similar than any pair of nonmates ever encountered, and that they are comparable in similarity to the known mates previously experienced. If so, this examiner would regard the pair as originating from the same individual. We can designate this approach as the mate-nonmate theory of source attribution. ${ }^{64}$ It assumes that the difficulty of classifications in the previously experienced sets of mated and nonmated prints are comparable to all those that ever will be encountered in casework. ${ }^{65}$

\footnotetext{
${ }^{59}$ For other discussions of this classic "Birthday Problem" in probability theory and forensic science, see, for example, NAS, NRC, Committee on DNA Forensic Science: An Update, op. cit., pp. 137, 161, 165; Page, M., J. Taylor, and M. Blenkin. "Uniqueness in the Forensic Identification Sciences-Fact or Fiction?" Forensic Science International, 206 (2011): 12, 1415.

${ }^{60}$ SWGFAST, 2011, op. cit.

${ }^{61}$ Butt, L. Letter from the Chairman, Scientific Working Group on Friction Ridge Analysis, Study and Technology. In Regards to: Position Clarification. June 29, 2010. Accessed June 3, 2011. http://www.swgfast.org/CommentsPositions/SWGFAST_NAS_Position_Clarification.doc.

${ }^{62}$ Scientific Working Group on Friction Ridge Analysis, Study and Technology. Draft for Comment: Standards for Examining Friction Ridge Impressions and Resulting Conclusions (To Replace: Friction Ridge Examination Methodology for Latent Print Examiners, Version 1.01, and Standards for Conclusions, Version 1.0). Version 1.0. Scientific Working Group on Friction Ridge Analysis, Study and Technology, August 2010, p. 4.

${ }^{63}$ Ibid., p. 5.

${ }^{64}$ Association of Firearm and Tool Mark Examiners. Theory of Identification as it Relates to Toolmarks. 5th ed., ver. 5.070207. 2007.

${ }^{65}$ Although thinking about the features of mated versus nonmated prints might describe how the examiner thinks (at some level), it does not respond to the criticism that it is difficult to say how much confidence one can place on
} 
Another possible route to identification that also does not rest on the theory of universal individualization has been called "local individualization" because it only claims that the source is very probably unique within a particular population that is much smaller than the entire human race. ${ }^{66}$ Local individualization is the assertion that a particular individual within a set of plausible suspects is the source of a latent print. This set might be enumerated (a "closed" list of suspects); alternatively, it might not be subject to explicit enumeration (the list is "open" even though it might be limited by geography or other factors). For example, the relevant population might be every resident of a dormitory who was in the dormitory on a given night ("closed"), or it might be everyone in a city ("open"). In a local individualization, the examiner concludes that the latent print came from one source even if he has not examined the prints of everyone else in this local population.

In Chapter 3, the Working Group recommends against presenting a latent print identification as meaning that the source of the exemplar is the source of the latent print to the exclusion of all other individuals in the world. However, the members of the Working Group have varying opinions as to whether the practice of making source attributions based on the mate-nonmate theory or the local-individualization theory is appropriate. Chapter 6 therefore describes alternatives to source-attribution testimony without endorsing any of them.

In sum, the term "identification" is not necessarily synonymous with "individualization." As a general matter, "identification" refers to the association, to some degree of probability, of a trace to a source. (The operation of probability theory in this context is explained in the next section.) One type of identification is the attribution of a trace to a single source (a source attribution). In latent print work, source attributions have traditionally rested on a theory of universal individualization. In this framework, an individualization is a statement that a single individual must be the source of the trace because no other individual in the world could have produced such a trace. Source attributions also can rest on less extreme theories of why the identified individual is, very probably, the source, and evidence that does not warrant a source attribution also can be useful in investigations and prosecutions.

\subsection{Probability}

In describing the nature of error (Chapter 2), the interpretive process of ACE-V (Chapter 3), emerging technologies (Chapter 4), and courtroom testimony (Chapter 6), this report uses terms and concepts from the theory of probability. Indeed, the previous section describes a source attribution as a statement that a particular object or individual is "very probably" the source of a trace. This section provides definitions of several of the terms used in theory of probability and indicates more fully how source attribution relates to probabilities. ${ }^{67}$

judgments made in this fashion if "[n]one of these variabilities — of features across a population of fingers or of repeated impressions left by the same finger - has been characterized, quantified, or compared." NAS, NRC, Committee on Identifying the Needs of the Forensic Science Community, op . cit.

${ }^{66}$ The logic of "local individualization" and "specific uniqueness" is described more fully in Chapter 6, in Kaye, Bernstein, and Mnookin, op. cit., and in Kaye, 2009, op. cit.

${ }^{67}$ For more extensive discussions, see, for example, Aitken, C. and F. Taroni. Statistics and the Evaluation of Evidence for Forensic Scientists. 2nd ed. John Wiley \& Sons, 2004; Kaye, Bernstein, and Mnookin, op. cit.; and Robertson, B. and G. Vignaux. Interpreting Evidence: Evaluating Forensic Science in the Courtroom. John Wiley \& Sons, 1995. 
Probability is a number between zero and one. A probability of zero means that a proposition is definitely false. A probability of one means that a proposition is certainly true. In the empirical sciences, absolute certainty in unattainable, although some propositions, such as the claim that the Earth orbits the Sun, have probabilities very close to one.

Probability often is expressed as a percentage. Thus, a $60 \%$ probability and a 0.6 probability are the same thing. Another way to describe uncertainty is with odds. If the probability that a particular baseball team will win the World Series is $60 \%$, the odds are 60 to 40 (or, equivalently, 1.5 to 1$)$.

To place the findings and inferences of forensic scientists in a probabilistic framework, it is necessary to distinguish between data or observations on the one hand, and hypotheses on the other. That a specific finger is the source of an impression is a hypothesis. The observed features in a pair of fingerprints are data. The data can make the hypothesis more probable or less probable than it otherwise would be. The probability before obtaining particular data is known as the prior probability. The probability after considering the data is known as the posterior probability.

The precise relationship between the prior and posterior probability is given by a formula known as Bayes's rule. The rule tells us how to update the prior probability in light of the data. When there are only two possible hypotheses to consider - such as the hypothesis that two impressions come from the same fingers and the competing hypothesis that they come from different fingers - the increase or decrease in the probability depends on a single factor, called the likelihood ratio. Specifically, the posterior odds are the prior odds multiplied by the likelihood ratio (see Box 1.3 for examples). The likelihood ratio thus measures the strength of the evidence. A large likelihood ratio means that the evidence is powerful: it raises the odds by a large factor.

The likelihood ratio is just the probability of the data when one hypothesis is true compared to (divided by) the probability of the data when the competing hypothesis is true. For example, when the features in a latent print and an exemplar are ten thousand times more probable for mates than nonmates, the likelihood ratio is ten thousand. Observing these features boosts the prior odds for identity (whatever they may be) by a factor of ten thousand. In principle, at least, an examiner could estimate the likelihood ratio subjectively or could compute it using a statistical model (see Chapters 3, 4, and 6). 


\section{Box 1.3: Probabilities, Likelihood Ratios, and Individualization}

The term "individualization" can be given various probabilistic interpretations. The traditional notion of universal individualization means that the probability of finding another matching exemplar (if exemplars existed for everyone in the world) is essentially zero. However, probability theory shows how evidence that is not individualizing in this traditional sense still can be extremely powerful.

As indicated in the text, probabilities can be assigned to two different hypotheses: (1) that two impressions come from the same finger $(s)$, and (2) that they come from different fingers $(d)$. If $\mathrm{P}_{e \mid s}$ denotes the probability of the observations (the evidence from the latent print examination) when the prints are from the same source, and $\mathrm{P}_{e \mid d}$ represents the probability when they are from different sources, then a simple measure of the extent to which the observations support the former hypothesis over the latter is the likelihood ratio, $\mathrm{LR}=\mathrm{P}_{e \mid s} / \mathrm{P}_{e \mid d^{*}}$

According to Bayes's rule, posterior odds $=L R \times$ prior odds. Suppose the likelihood ratio is one million, meaning that an apparent match is one million times more probable if the prints come from the same source than if they come from different sources. Although this likelihood ratio seems extremely high, if we assume for the sake of argument that all residents of New York City were equally likely to have made an impression, then the odds of $s$ to $d$ change from one to eight million (before considering the evidence) to one to eight (after considering the evidence).

For suspect populations of various sizes, the posterior odds of identity for evidence with this likelihood ratio are as follows:

\begin{tabular}{|l|l|}
\multicolumn{1}{|c|}{$\begin{array}{c}\text { Population } \\
\text { size }\end{array}$} & \multicolumn{1}{|c|}{$\begin{array}{c}\text { Posterior odds of probability } \\
(\mathbf{L R}=\mathbf{1 , 0 0 0 , 0 0 0})\end{array}$} \\
\hline The world $(\sim 7,000,000,000)$ & $1: 7,000$ \\
\hline United States $(\sim 300,000,000)$ & $1: 300$ \\
\hline New York City $(\sim 8,000,000)$ & $1: 8$ \\
\hline Colorado Springs $(\sim 400,000)$ & $2.5: 1$ \\
\hline Walla Walla $(\sim 30,000)$ & $33: 1$ \\
\hline A college dormitory $(\sim 200)$ & $5,025: 1$ \\
\hline
\end{tabular}

Table 1.2: Posterior odds of identity for evidence with a likelihood ratio of 1,000,000 in populations in which everyone has the same prior odds on being the source of a latent print

An examiner who makes a source attribution is giving an opinion that the posterior probability is practically $1(100 \%)$.

\subsection{Summary}

This chapter defines various terms and concepts that are central to the assessment of latent print evidence. In particular, it describes the steps of the ACE-V process as currently practiced and understood by the latent print examination community and discusses how exclusions and identifications are fundamentally statements of probabilities. Because humans conduct the process, human factors can influence the outcome of an examination. Bias and error can occur in any process for making comparisons and drawing inferences. In the context of latent print examination, they can play a role in the final decision made by an examiner. Many other human factors are at play in the latent print work environment, as addressed in the chapters that follow. 


\section{Chapter 2: Human Factors and Errors}

Human beings, in all lines of work, make errors. Errors can be prevented by designing systems that make it hard for people to do the wrong thing and easy for people to do the right thing. Cars are designed so that drivers cannot start them while in reverse because that prevents accidents. Work schedules for pilots are designed so they don't fly too many consecutive hours without rest because alertness and performance are compromised.

$$
\text { -To Err Is Human: Building A Safer Health System }{ }^{68}
$$

\section{Introduction and Scope}

One goal of this chapter on human factors and errors in latent print examination is to explain the systems view of human factors. What is true of drivers and pilots - not to mention judges, police officers, lawyers, soldiers, and scholars - is also true of forensic scientists and technicians. All of us make errors. This report makes no effort to hide this fact. To the contrary, the Working Group agrees that the "culture of blame must be broken down, [and] we must systematically design safety into processes." ${ }^{\prime 69}$ Later chapters apply these ideas to matters such as the training and working environment of examiners as well as their performance in interpreting evidence and reporting and testifying about their findings.

The other major goal of this chapter is to describe methods for quantifying the risk of errors in latent print examinations. Measuring the incidence of errors serves three functions. First, studying error is an integral part of science. A basic tenet of experimental science is that "errors and uncertainties exist that must be reduced by improved experimental techniques and repeated measurements, and those errors remaining must always be estimated to establish the validity of our results." ${ }^{70}$ What applies to physics and chemistry applies to all of forensic science: "A key task ... for the analyst applying a scientific method is to conduct a particular analysis to identify as many sources of error as possible, to control or eliminate as many as possible, and to estimate the magnitude of remaining errors so that the conclusions drawn from the study are valid." ${ }^{\prime \prime 1}$ In other words, errors should, to the extent possible, be identified and quantified.

Second, measuring and tracking error rates is part of a comprehensive quality control and assurance system. This idea is not new. In the field of medicine, it has been called the "error movement," and it has firm roots in industrial psychology. ${ }^{72}$ Although it is difficult to

\footnotetext{
${ }^{68}$ National Academy of Sciences, Institute of Medicine, Committee on Quality of Health Care in America. To Err Is Human: Building A Safer Health System. National Academies Press, 1999, p. ix.

${ }^{69} \mathrm{Ibid}$.

${ }^{70}$ Bevington, P. and D. Robinson. Data Reduction and Error Analysis for the Physical Sciences. 3rd ed. McGrawHill, 2002, p. 1.

${ }^{71}$ National Academy of Sciences, National Research Council, Committee on Identifying the Needs of the Forensic Science Community. Strengthening Forensic Science in the United States: A Path Forward. National Academies Press, 2009, p. 111.

${ }^{72}$ See, e.g., NAS, Institute of Medicine, op. cit.; and Hofer, T., E. Kerr, and R. Hayward. "What Is an Error?" Effective Clinical Practice, 3 (2000): 261.
} 
demonstrate that the medical error movement has improved hospital patient safety in general, ${ }^{73}$ quantitative analysis can identify factors associated with errors - including preventable onesand thus suggest strategies for error reduction. ${ }^{74}$ Chapter 9 discusses the importance of implementing effective systems to detect deviations from desired practices and incorrect judgments in latent print casework.

Finally, in the legal system, quantified "error rates" have long been a consideration in judging the admissibility of findings or the weight that should be given to them. Quantification not only can lead to improvements in the reliability and validity of current practices, but it also could assist in more appropriate use of the evidence by fact-finders. The objective, in other words, is improving the "back end" of the systemthe use of the evidence in investigations and legal proceedings. Many court opinions have discussed error rates of scientific tests such as polygraphy, speaker identification, and latent print identification as a consideration affecting the admissibility of these tests. To ascertain the prospects for useful quantification, however, there must be clarity about the meaning of the surprisingly ambiguous term "error" as it applies to the task of latent print identification. It is necessary to consider various ways to measure a suitably defined "error rate" that can

Box 2.1: Daubert v. Merrell Dow Pharmaceuticals, Inc., and "Error Rates"

In Daubert v. Merrell Dow Pharmaceuticals, Inc. ${ }^{75}$ the Supreme Court wrote that federal courts should determine whether contested scientific evidence is "scientifically valid." It listed four non-exhaustive factors that trial judges could consider in making these determinations. One of these factors came from a federal court of appeals opinion on spectrographic voice identification that relied on laboratory experiments measuring the rate of false positives and false negatives. Citing this case, the Supreme Court stated, "In the case of a particular scientific technique, the court ordinarily should consider the known or potential rate of error." 76

The rules of evidence in many states treat "general acceptance" in the scientific community rather than "scientific validity" as the touchstone for admitting scientific evidence. Courts in these jurisdictions have treated error rates from controlled experiments as a factor that can help show whether a technique is generally accepted. be used to assess the validity of the method and conclusions.

\subsection{The Systems View of Errors and Human Factors}

The systems view of human error regards errors and adverse events as a function of a system of interacting parts, any or all of which could present opportunities for preventing and correcting errors. Forensic science evidence results from a complex productive system. A single examiner conducts latent print examinations using various technological tools, but more than one individual participates in the production process. A detective may provide candidate suspect prints, and an examiner may run a search of an automated fingerprint identification system (AFIS) that produces both viable matches and nonmatches that initially appear similar. Finally,

\footnotetext{
${ }^{73}$ Landrigan, C., G. Parry, C. Bones, et al. "Temporal Trends in Rates of Patient Harm Resulting from Medical Care.” New England Journal of Medicine, 363 (2010): 2124.

${ }^{74}$ Macklis, R., T. Meier, and M. Weinhous. "Error Rates in Clinical Radiotherapy." Journal of Clinical Oncology, 16 (1998): 551.; and Weingart, S., R. Wilson, R. Gibberd, et al. "Epidemiology of Medical Error.” British Medical Journal, 320 (2000): 774.

${ }^{75}$ Daubert v. Merrell Dow Pharmaceuticals, Inc., 509 U.S. 579 (1993).

${ }^{76}$ Id. at 594.
} 
additional individuals may verify work. Thus, the etiology of errors requires a characterization of not only the contributions of each state of the process to potential errors, but also their interactions, since interactions among parts of the system can serve to reduce errors or contribute to them. Simply blaming errors on individuals is simplistic and unproductive. One must appreciate how human actors function in and interact with other components of a more complex system.

The "Swiss cheese" model of errors (see Figure 9.2 in Chapter 9) ${ }^{77}$ which allows classifications of various human factors that contribute to errors, provides a framework for identifying weaknesses in the examination process. Briefly, this framework involves factors that are specific to an individual examiner, those that are features or products of the work environment, and those that are the result of failures of supervision and the structure of the organization. This model is explained in more detail in Chapter 9. A seminal example of the effects of interactions between humans and technology comes from Hutchins, ${ }^{78}$ who characterized the tools, procedures, training, and checklists that allow a cockpit crew, working with tools such as airspeed indicators, to ensure that an airplane does not stall on landing approach. The indicators and control surface settings provide valuable safety margins and checks, but they can also can lead to crashes if set incorrectly.

Similarly, latent print examination may well benefit from procedures such as verification, but if such procedures are implemented improperly, they can lead to a mere illusion of error reduction. Not knowing when an examiner has erred makes error reduction difficult. Numerous studies have found that without quick and accurate feedback on correct and incorrect judgments, experience does not enhance expertise ${ }^{79}$ and that experts routinely overestimate their skills. ${ }^{80}$ Weather forecasters are particularly well calibrated; when they report that the chance of rain is $60 \%$, it rains about $60 \%$ of the time. Lawyers and doctors are not so well calibrated; their predictions of the outcomes of cases tend to be optimistic. ${ }^{81}$ The explanation for the difference, at least in part, is that meteorologists get frequent, immediate feedback and rewards for correct predictions. ${ }^{82}$ In forensic handwriting analysis, latent print identification, toolmark identification, and other pattern and impression evidence comparisons, examiners do not routinely receive such prompt and frequent feedback. ${ }^{83}$ Under what circumstances would examiners realize that they are working with prints that are beyond their current capabilities? If an examiner feels that his

\footnotetext{
${ }^{77}$ This model is presented in Reason, J. Human Error. Cambridge University Press, 1990. It is refined in Wiegmann, D. and S. Shappell. A Human Error Approach to Aviation Accident Analysis: The Human Factors Analysis and Classification System. Ashgate Publishing, 2003.

${ }^{78}$ Hutchins, E. "How a Cockpit Remembers Its Speeds.” Cognitive Science, 19 (1995): 265

${ }^{79}$ E.g., Bazerman, M. and M. Neale. Negotiating Rationally. Free Press, 1994, p. 102; and Tversky, A. and D. Kahneman. "Rational Choice and the Framing of Decisions." Journal of Business, 59 (1986): 251.

${ }^{80}$ Hoffrage, U. "Overconfidence." In Cognitive Illusions: A Handbook on Fallacies and Biases in Thinking, Judgment, and Memory. Psychology Press, 2004; and Lichtenstein, S., B. Fischhoff, and L. Phillips. "Calibration of Probabilities: The State of the Art to 1980." Judgment Under Uncertainty: Heuristics and Biases. Cambridge University Press, 1982.

${ }^{81}$ Goodman-Delahunty, J., P. Granhag, M Hartwig, et al. "Insightful or Wishful: Lawyers' Ability to Predict Case Outcomes.” Psychology, Public Policy, and Law, 16 (2010): 133.

${ }^{82}$ See National Academy of Sciences, National Research Council, Committee on Behavioral and Social Science Research to Improve Intelligence Analysis for National Security. Intelligence Analysis for Tomorrow: Advances from the Behavioral and Social Sciences. National Academies Press, 2011.

${ }^{83}$ Kaye, D., D. Bernstein, and J. Mnookin. The New Wigmore, A Treatise on Evidence: Expert Evidence. 2nd ed. Aspen Publishers, 2011.
} 
abilities far outstrip his colleagues' and is willing to make identifications that they would not, how would such a situation be identified and resolved? Procedures to reconcile differences of opinion might only reduce the independence of each examiner without moving the decision criterion toward an optimum.

Thus, procedural changes aimed at error reduction must be devised and examined at a systemwide level. These changes must emphasize data collection and feedback to allow both the agency and the examiners to adjust their decision criteria and methods. Well-designed proficiency tests, employed to enhance skills, are one way to supply feedback. Excluding unnecessary contextual information that might affect the outcome of an examination is another strategy best implemented with an appreciation of how the system works, since such information may need to be integrated at later stages without affecting the initial examination.

Detecting, analyzing, quantifying, and reducing error is difficult, but these efforts are important quality assurance steps. To take these steps, however, it is necessary to define terms such as "error" precisely. The remainder of this chapter, therefore, analyzes the meaning of "error," elucidates some possible types and sources of error in the Analysis, Comparison, Evaluation, and Verification (ACE-V) process, and compares methods for estimating the risk of error in latent friction ridge identification as performed by examiners following ACE-V.

\subsection{Errors in Executing a Procedure}

When an individual is expected to follow well-defined steps in a procedure, a departure from the protocol or a mistake in one of the steps might be designated an error. For example, suppose that a forensic service provider requires its examiners to mark the useful features in a latent print before making a side-by-side comparison to an exemplar. A particular examiner neglects to do so, but correctly determines that the differences between the latent images and the exemplar are so extensive and clear that the prints could not have originated from the same source. The examiner then correctly excludes the suspect from whom the exemplar came.

This examiner has not erred in the sense of reaching a false conclusion, but the examiner has erred in deviating from the prescribed procedure. Identifying such procedural errors (also called "process errors" in medicine) ${ }^{84}$ perhaps through audits of casework, could be valuable even when the errors did not lead to a false conclusion. If the procedural step serves a useful function in general, then discovering that examiners are not performing the step may warrant corrective action. For example, the system might be redesigned so that examiners must sign a checklist as they go along to remind them to complete each step in order. ${ }^{85}$

If an agency has a policy demanding blind verification of identifications, another example of procedural error would be providing the verifying examiner with the knowledge that an earlier examination conducted by a highly respected colleague resulted in an identification. Such policies serve to prevent any "expectancy effect" from influencing the verification (see Chapters

\footnotetext{
${ }^{84}$ Hickner, J., D. Graham, N. Elder, et al. "Testing Process Errors and Their Harms and Consequences Reported From Family Medicine Practices: A Study of the American Academy of Family Physicians National Research Network." Quality and Safety in Health Care, 17 (2008): 194.

${ }^{85}$ Compare Stevenson, K., S. Gibson, D. MacDonald, et al. "Measurement of Process as Quality Control in the Management of Acute Surgical Emergencies.” British Journal of Surgery, 95 (2007): 376.
} 
1 and 3). In a laboratory that requires blind verification, a nonblind verification would be a procedural error. Discovering a large rate of such departures from the established protocol should produce an inquiry into what should be done to correct the situation.

\subsection{Errors in Outcomes}

Another type of error, an outcome error, contrasts with procedural error. It is closer, but not identical, to an "adverse event" in fields such as aviation and medicine ${ }^{86}$ and relates to reporting the wrong conclusions in latent print examination. Bias does not always produce an incorrect outcome. For instance, a radar gun that gives readings that are always 2 miles per hour too low is biased, but a reading of $45 \mathrm{mph}$ is a good indication that a driver is exceeding the speed limit of $35 \mathrm{mph}$ (at least when the random error also is small relative to the measured excess of $10 \mathrm{mph}$ ). In this example, the performance of the measuring device can be compared to the true state of affairs - what sometimes is called "ground truth" in biometrics ${ }^{87}$ or "states of nature" in decision theory ${ }^{88}$ However, in real-world latent print casework, this true state of affairs is rarely available.

Human decision making also can be poorly calibrated or well calibrated in terms of its accuracy in ascertaining the true state of affairs. If a latent print and an exemplar come from two different individuals, will an examiner (or an initial examiner and a verifier) ${ }^{89}$ report that the two prints match? If the two prints come from the same individual, will the examiner report that they do not match? Of the positive identifications made by examiners, what percentage is mistaken? Of the exclusions, what percentage is incorrect? Each of these questions leads to a different statisticthere is no single "error rate" and no single number that gives the "accuracy" of judgments about the origin of pairs of prints. ${ }^{90}$ Moreover, as discussed below, further questions arise when one considers an examiner's determination that a latent print is insufficient to warrant further examination or that the further examination is inconclusive.

The simplest measures of accuracy (or, conversely, error) in categorization arise when there are only two categories. Putting aside, for the moment, judgments of "insufficient" or "inconclusive," the examiner reports either that the prints match or that they do not. The same kind of binary categorization task is central to many other disciplines. ${ }^{91}$ Clinical diagnosis, predictions of future violence, and signal detection are a few examples. A radar operator may

\footnotetext{
${ }^{86}$ E.g., Hofer, Kerr, and Hayward, op. cit.

${ }^{87}$ E.g., Ratha, N., S. Chen, and A. Jain. “Adaptive Flow Orientation-Based Feature Extraction in Fingerprint Images.” Pattern Recognition, 28 (1995): 1657.

${ }^{88}$ Berger, J. Statistical Decision Theory and Bayesian Analysis. 2nd ed. Springer, 1984, p. 3.

${ }^{89}$ For ease of exposition, the explication of the concept of error in inferring identity that follows speaks of a single examiner. The final categorization could result from one examiner using the ACE phases of the process (or any other process) or from several examiners using ACE-V (or any other process).

${ }^{90}$ E.g., Kaye, Bernstein, and Mnookin, op. cit.

${ }^{91}$ In the terminology of the 2009 NRC report and statistics and measurement theory generally, latent print identification as performed through the steps of ACE-V is an act of classification. To avoid confusing the yesno type of classification for identification in latent print work with ten-print classification systems (such as the Roscher system, the Juan Vucetich system, and the Henry system), however, this chapter generally uses the term "categorization." See Allen, M. and W. Yen. Introduction to Measurement Theory. Waveland Press, 2001; Suppes, P. and J. Zinnes. "Basic Measurement Theory." In Handbook of Mathematical Psychology. Wiley, 1963; and Rothman, K., S. Greenland, and T. Lash. Modern Epidemiology. 3rd ed. Lippincott, Williams \& Wilkins, 2008, p. 138.
} 
need to distinguish between a missile launch, on the one hand, and a flock of birds or anything else on the other. Signal-detection theory provides a well-developed analytical framework involving the probabilities of false alarms (false positives) and missed signals (false negatives).$^{92}$

The definition of error in this analytical framework is simple. The outcome is judged against a true state of the world. An error is a reported categorization that does not report the true state. If the radar operator misses the signal of the missile launch (a false negative), or if the operator declares an alarm when no missile is in the air (a false positive), then the error will become apparent all too soon. ${ }^{93}$ Table 2.1 summarizes the same two kinds of errors that can arise in determining whether a latent print is from the known source of an exemplar. The first column lists the examiner's possible decisions: declare that the prints are so different that the source of the exemplar is excluded (abbreviated as -) or declare that the prints are so similar that the source of the exemplar is included (an identification, abbreviated as + ). The first row lists the true states of the world: different source $(d)$ or same source $(s)$. In casework, these states would not generally be known. In an experiment or a proficiency test, however, they would be. This knowledge makes no difference to the definition of an outcome error, although it affects our ability to estimate the conditional error probabilities. In the table, "P( )" stands for "probability of." The vertical bar "|" stands for "given that" or "on the condition that."

\begin{tabular}{|l|l|l|}
\cline { 2 - 3 } \multicolumn{1}{c|}{} & \multicolumn{1}{c|}{ Different source $(\boldsymbol{d})$} & \multicolumn{1}{c|}{ Same source $(s)$} \\
\hline Exclusion $(-)$ & $\begin{array}{l}\text { True negative } \\
\text { Specificity }=\mathrm{P}(-\mid d)\end{array}$ & $\begin{array}{l}\text { False negative (a miss, type II } \\
\text { error) } \\
1-\text { sensitivity }=\mathrm{P}(-\mid s)\end{array}$ \\
\hline $\begin{array}{l}\text { Identification } \\
(+)\end{array}$ & $\begin{array}{l}\text { False positive (false alarm, type I } \\
\text { error) } \\
1-\text { specificity }=\mathrm{P}(+\mid d)\end{array}$ & $\begin{array}{l}\text { True positive }(\text { a hit) } \\
\text { Sensitivity }=\mathrm{P}(+\mid s)\end{array}$ \\
\hline
\end{tabular}

Table 2.1: Types of errors and correct conclusions in a binary classification task

In latent print comparisons that lead to only the two outcomes shown in Table 2.1 - exclusions and identifications - a false negative error is an exclusion when the pair actually originated from the same friction ridge skin. Its probability, marked in the upper right-hand corner of the table, is $\mathrm{P}(-\mid s)$. In contrast, a false positive probability $\mathrm{P}(+\mid d)$, in the lower left-hand corner of the table, measures the chance of an identification when the pair actually did not originate from the same source.

Instead of referring to these two conditional error probabilities, one can refer to two related quantities that define the accuracy of the judgment. Sensitivity is the probability of identification

\footnotetext{
${ }_{92}$ Melsa, J. and D. Cohn. Decision and Estimation Theory. McGraw-Hill, 1978; Swets, J. and R. Pickett. Evaluation of Diagnostic Systems: Methods from Signal Detection Theory. Academic Press, 1982; and Wickens, T. Elementary Signal Detection Theory. Oxford University Press, USA, 2002.

${ }^{93}$ When the categorization system maps quantitative scores into binary categories, its performance can be depicted as a receiver operating characteristic (ROC) curve. This is a plot of the true positive rate (sensitivity) vs. false positive rate ( 1 - specificity) for a binary classifier system as its discrimination threshold is varied. National Academy of Sciences, National Research Council, Committee on Evaluation of Sound Spectrograms. On the Theory and Practice of Voice Identification. National Academies Press, 1979, p. 4.
} 
given that the prints actually come from the same source. These correct outcomes lie in the bottom right-hand corner of the table. Specificity is the probability of exclusion given that the prints actually came from different sources (upper left-hand corner).

Sensitivity and specificity are commonly used to appraise the diagnostic value of a test in clinical medicine. A pregnancy test that almost always gives a positive result in cases of pregnancy has a high sensitivity. On the other hand, a pregnancy test that identifies many women as pregnant when they are not has low specificity. That pregnancy test is sensitive to the condition but its positive results are not very specific to pregnancy. An ideal diagnostic test is both highly sensitive and highly specific, and so is the ideal fingerprint examiner. Encountering a latent and an exemplar from the same source, this examiner almost always makes an identification. Presented with a latent and an exemplar from different sources, this examiner almost always makes an exclusion.

The important points to be gleaned from Table 2.1 are that (1) each type of error has a probability of occurring given the true state of the world (whether known or unknown), and (2) the falsepositive probability is not generally equal to the probability that the prints are from different individuals when an examiner declares a match. The latter is a posterior probability (Chapter 1). To make this more concrete, suppose that an experimenter gives an examiner 200 pairs of prints to examine. A latent and an exemplar comprise each pair. For 100 of the pairs, the latent and the exemplar come from different individuals $(d)$; and for the other 100 pairs, the latent and the exemplar come from the same individuals $(s)$. The examiner compares the prints in each and every pair, and correctly classifies 99 of the former group and 98 of the latter. This situation is shown in Table 2.2. Again, these numbers are hypothetical and merely intended to illustrate how various measures of accuracy and error are computed. Results from a recent experiment are described later in this chapter.

\begin{tabular}{|l|l|l|}
\cline { 2 - 3 } \multicolumn{1}{c|}{} & \multicolumn{1}{c|}{ Different source $(\boldsymbol{d})$} & \multicolumn{1}{c|}{ Same source $(\boldsymbol{s})$} \\
\hline Exclusion $(-)$ & $\begin{array}{l}\text { True negatives }=99 \\
\text { Specificity }=\mathrm{P}(-\mid d)=99 \%\end{array}$ & $\begin{array}{l}\text { False negatives }=2 \\
1-\text { sensitivity }=\mathrm{P}(-\mid s)=2 \%\end{array}$ \\
\hline $\begin{array}{l}\text { Identification } \\
(+)\end{array}$ & $\begin{array}{l}\text { False positives }=1 \\
1-\text { specificity }=\mathrm{P}(+\mid d)=1 \%\end{array}$ & $\begin{array}{l}\text { True positives }=98 \\
\text { Sensitivity }=\mathrm{P}(+\mid s)=98 \%\end{array}$ \\
\hline
\end{tabular}

\section{Table 2.2: Outcomes of a hypothetical experiment that estimates an examiner's sensitivity and specificity}

The examiner in this example has performed with a sensitivity of $98 \%$ (correct identifications) and a specificity of $99 \%$ (correct exclusions). Equivalently, the examiner's false negative and false positive rates were $2 \%$ and $1 \%$, respectively.

It is important to understand that these numbers do not translate directly into a probability that an identification or an exclusion is correct. The probabilities of a correct identification or exclusion are influenced by the proportion of cases in which the pairs of prints actually come from the same individuals. Box 2.2 presents a numerical example of how changing the mix of true and false pairs changes the probability of a true positive identification. 


\section{Box 2.2: How Prevalence, Sensitivity, and Specificity Affect the Posterior Probability of a Correct Positive Identification}

The probability that the examination has correctly classified a pair of prints as identifying the individual is the proportion of all those cases in which the examiner has made a positive identification that involve true pairs. For example, consider a set of 1,000 pairs of prints that includes only 800 true pairs for an $80-20$ mix. An examiner with a sensitivity of $98 \%$ would classify approximately $98 \%$ of these 800 , or 784 , as identifications. But there also are 200 false pairs to consider. The examiner with a specificity of $99 \%$ would classify approximately $1 \%$ of these 200 , or 2 , as identifications. The total number of identifications therefore would be $784+2=786$. Thus, the examiner would make correct identifications for approximately 784 out of 786 identifications: $\mathrm{P}(s \mid+)=784 / 786=99.7 \%$. The following table summarizes these calculations:

\begin{tabular}{|l|r|r|c|}
\cline { 2 - 4 } \multicolumn{1}{c|}{} & Number & Number + & $\operatorname{Pr}\left(\left.\boldsymbol{s}\right|^{+}\right)$ \\
\hline True pairs $(\boldsymbol{s})$ & 800 & 784 & \\
\hline False pairs $(\boldsymbol{d})$ & 200 & 2 & \\
\hline Total & 1,000 & 786 & $784 / 786=99.7 \%$ \\
\hline
\end{tabular}

Table 2.3: Hypothetical data to show probability of correct identifications in an 80-20 mix

Now suppose that the examiner's casework consisted of a 10-90 mix of true and false pairs. Of the 1,000 cases, 100 are true pairs, and 900 are false pairs. This depresses the value of $\mathrm{P}(s \mid+)$ to $(100 \times 98 \%) /$ $[(100 \times 98 \%)+(900 \times 1 \%)]=98 /(98+9)=92 \%$. The examiner's ability to discriminate between a true pair and a false pair has not changed. The sensitivity is still $98 \%$, and the specificity is still $99 \%$. However, the expected number of each type of error in the sample of casework is different, lowering the probability that a positive identification is correct, as shown in the following table: ${ }^{94}$

\begin{tabular}{|l|r|r|c|}
\cline { 2 - 4 } \multicolumn{1}{c|}{} & Number & Number + & $\mathbf{P}(\boldsymbol{s} \mid+)$ \\
\hline True pairs $(\boldsymbol{s})$ & 100 & 98 & \\
\hline False pairs $(\boldsymbol{d})$ & 900 & 9 & \\
\hline Total & 1,000 & 107 & $98 / 107=92 \%$ \\
\hline
\end{tabular}

Table 2.4: Hypothetical data to show probability of correct identifications in a 10-90 mix

Thus far, we have assumed that the identification process always reaches a conclusion of exclusion or identification, and we have described how an experiment could measure the sensitivity and specificity of examiners using the ACE-V (or any other) process to reach these conclusions. However, examiners have other options. They can declare the latent print (or exemplar) to be insufficient for a comparison, or they can make a comparison but designate it as

\footnotetext{
94 These computations are examples of Bayes's rule, where the proportion of true pairs in casework is the prior probability that a random pair comes from the same source. Compare Parmigiani, G. Modeling in Medical Decision Making: A Bayesian Approach. Wiley, 2002, p. 9. In a fully Bayesian analysis, however, the prior probability is subjective and need not equal the past frequency. But even if there is no known value, the existence of a prior probability that a random pair comes from the same source has to be borne in mind. In that way, examiners are more likely to remember that the probabilities of exclusion or identification given a pair of impressions from the same source are not the probabilities that a source exclusion or attribution is correct.
} 
"inconclusive." Table 2.1 can be expanded with an additional row to account for those possible outcomes (Table 2.5).

\begin{tabular}{|l|l|l|}
\cline { 2 - 3 } \multicolumn{1}{c|}{} & \multicolumn{1}{c|}{ Different source $(\boldsymbol{d})$} & \multicolumn{1}{c|}{ Same source $(\boldsymbol{s})$} \\
\hline Exclusion $(-)$ & $\begin{array}{l}\text { True negative } \\
\text { Specificity }=\mathrm{P}(-\mid d)\end{array}$ & $\begin{array}{l}\text { False negative } \\
1-\text { sensitivity }=\mathrm{P}(-\mid s)\end{array}$ \\
\hline Identification $(+)$ & $\begin{array}{l}\text { False positive } \\
1-\text { specificity }=\mathrm{P}(+\mid d)\end{array}$ & $\begin{array}{l}\text { True positive }(\text { a hit }) \\
\text { Sensitivity }=\mathrm{P}(+\mid s)\end{array}$ \\
\hline $\begin{array}{l}\text { Insufficient or } \\
\text { inconclusive }\end{array}$ & Missed an exclusion & Missed an identification \\
\hline
\end{tabular}

Table 2.5: Types of errors and correct conclusions in a binary classification task with the option of not deciding

The additional row complicates the computation of error and accuracy rates. ${ }^{95}$ Should sensitivity and specificity be calculated relative to ground truth for all cases presented, or just for those in which an examiner reaches a definitive conclusion (an exclusion or an identification)? The argument for tossing out inconclusives is as follows:

When an examiner offers an "inconclusive" opinion about whether two prints match, there is a sense in which he has erred. After all, he did not get the answer right, and the consequences of this failure may be serious (e.g., missed opportunity to exonerate a suspect). However, in the more usual sense of the meaning of error, an inconclusive is not an error. It is a pass. An inconclusive means that the examiner offers no judgment about whether two prints do or do not share a common source. ${ }^{96}$

Suppose, then, that one agrees that an inconclusive cannot be considered an outcome error (not even an outcome error that is less serious than a false identification or a false exclusion). It is still not clear whether the inconclusives should be included in the denominators of the proportions for true and false positives and true and false negatives.

The answer may turn on the setting in which the error and accuracy rates will be used. The view that inconclusives should not count is appropriate from the perspective of a judge or juror who might consider error rates or probabilities to assess the probative value of an identification or an exclusion. For that purpose, it does not matter how often the examiner refrains from reaching a categorical conclusion. What matters is accuracy in those cases in which the examiner does offer an opinion on identification or exclusion. These are the only cases in which an examiner's testimony might lead the jury astray. Testimony that the latent print contained inadequate information to reach any conclusion as to the origin of the print occurs less often and should not

\footnotetext{
${ }^{95}$ See Scientific Working Group on Friction Ridge Analysis, Study and Technology. Draft for Comment: Standard for the Definition and Measurement of Rates of Errors and Inappropriate Decisions in Friction Ridge Examination. Version 1.0. Scientific Working Group on Friction Ridge Analysis, Study and Technology, February 2011.

${ }^{96}$ Koehler, J. "Fingerprint Error Rates And Proficiency Tests: What They Are And Why They Matter." Hastings Law Journal, 59 (2008): 1077.
} 
propel the jury in any particular direction. Therefore, any calculated error rate presented in a trial involving an identification or an exclusion should be based upon the subset of cases in which examiners actually make an identification or an exclusion.

Nevertheless, from the perspective of the police, what matters is all the cases that an examiner considers rather than just those in which the examiner ultimately might testify. In terms of improving the contribution of the examiner to the investigative process, it is appropriate to regard the failure to identify or exclude when the latent print contains adequate information as a potentially correctable error. Likewise, deciding that the latent print is of sufficient quality, but concluding that the comparison is inconclusive when, in fact, the similarities (or differences) are distinct and extensive, also is an error. Whether one regards such errors as outcome or process errors (or both), they are important because they might warrant a change in training or operational procedures to take fuller advantage of the latent friction ridge data. ${ }^{97}$

To help identify undesirable inconclusives, reliability and reproducibility studies could compare the performance of examiners in making judgments of sufficiency and conclusiveness. When the same examiner reaches the same conclusions (whether right or wrong) in repeated examinations of the same set of prints with no recollection of the earlier results, "intra-examiner reliability" is high. When different examiners independently studying the same set of prints reach the same conclusions, "inter-examiner reproducibility" is high. Table 2.6 uses four categories for the outcomes of latent print examinations: identification, exclusion, inconclusive, and insufficient (not suitable for comparison). A high number of discrepant judgments signals a need to understand what is causing the discordance and perhaps to correct it.

\begin{tabular}{|c|l|c|c|c|c|}
\cline { 3 - 6 } \multicolumn{2}{c|}{} & \multicolumn{4}{c|}{ Examiner 2 } \\
\cline { 3 - 6 } \multicolumn{2}{c|}{} & Identification & Exclusion & Inconclusive & Insufficient \\
\hline \multirow{2}{*}{} & Identification & AGREE & & & \\
\cline { 2 - 6 } & Exclusion & & AGREE & & \\
\cline { 2 - 6 } & Inconclusive & & & AGREE & \\
\cline { 2 - 6 } & Insufficient & & & & AGREE \\
\hline
\end{tabular}

Table 2.6: Concordancy in judgments of two examiners

A related method of spotting problematic judgments uses pairs of prints that are relatively complex to examine but that the agency is convinced should result in specific judgments in each category. In this situation, there are correct answers (in the judgment of the best experts), and the objective is to ensure that all examiners reach these desired outcomes. Table 2.7 shows the correct and incorrect outcomes relative to the desired outcomes rather than to ground truth.

\footnotetext{
${ }^{97}$ Because of the differing possible uses of error and accuracy rates, in discussing studies of these rates, this chapter gives the results of calculations both with and without inconclusives.
} 


\begin{tabular}{|c|c|c|c|c|c|}
\hline & \multicolumn{4}{|c|}{ Desired Outcomes as Determined by Experts } \\
\hline & & Identification & Exclusion & Inconclusive & Insufficient \\
\hline \multirow{4}{*}{ 预 } & Identification & CORRECT & False positive & False positive & False positive \\
\hline & Exclusion & False negative & CORRECT & False negative & False negative \\
\hline & Inconclusive & $\begin{array}{c}\text { Missed an } \\
\text { identification }\end{array}$ & $\begin{array}{l}\text { Missed an } \\
\text { exclusion }\end{array}$ & CORRECT & $\begin{array}{c}\text { Missed } \\
\text { insufficient }\end{array}$ \\
\hline & Insufficient & $\begin{array}{c}\text { Missed an } \\
\text { identification }\end{array}$ & $\begin{array}{l}\text { Missed an } \\
\text { exclusion }\end{array}$ & $\begin{array}{c}\text { Missed an } \\
\text { inconclusive }\end{array}$ & CORRECT \\
\hline
\end{tabular}

Table 2.7: Concordancy with desired outcomes as determined by experts

The discordant outcomes in Table 2.7 are of varying importance. Many of them would not affect the usefulness of the examiner's findings. For example, if an examiner concludes that the prints are insufficient for comparison when the "correct" opinion is that they are worth further study but are nonetheless inconclusive, then the examiner did not produce a result that could have affected the outcome of that case. Nonetheless, an examiner who is too prone to dismiss samples as unsuitable when they are sufficient for comparison but ultimately inconclusive also might be too inclined to regard them as insufficient when they would permit an identification or an exclusion. These discordant outcomes may not produce outcome errors, and they are not amenable to inclusion in "error rates" for ascertaining probative value. But following up on them could be important to making the best use of the data and to reducing the risk of actual outcome errors.

\subsection{Measuring the Prevalence of Error: Prospects and Pitfalls}

On occasion, the lures and pressures of the adversarial system, combined with the natural tendency of scientists and technicians to be confident in the power of the technology at their command and their decision-making abilities, can lead to excessive claims of accuracy. Two examples unrelated to friction ridge identification illustrate the phenomenon. First, claims that "everyone's voiceprint is as unique as his fingerprints, and that any skilled technician can identify a voiceprint with more than $99 \%$ accuracy," ${ }^{98}$ prompted the National Research Council (NRC) to caution that "the assumption that intra-speaker variability is less than ... inter-speaker variability ... is not adequately supported by scientific theory and data." 99 Second, in the initial years of DNA testing, expert witnesses claimed that false positive matches were impossible. ${ }^{100}$ In response, a separate NRC committee reminded them that "laboratory errors happen, even in the best laboratories and even when the analyst is certain that every precaution against error was taken." 101

\footnotetext{
${ }^{98}$ Kersta, L. As summarized in "The Law: Speak, Voiceprint.” Time. January 10, 1972.

${ }^{99}$ NAS, NRC, Committee on Evaluation of Sound Spectrograms, op.cit.

${ }^{100}$ For examples, see Kaye, D. "Probability, Individualization, and Uniqueness in Forensic Science Evidence: Listening to the Academies." Brooklyn Law Review, 75 (Summer 2010): 1163.

${ }^{101}$ National Academy of Sciences, National Research Council, Committee on DNA Technology in Forensic Science. DNA Technology in Forensic Science. National Academies Press, 1992, p. 82.
} 
Expert witnesses on latent print identification have heeded these admonitions. To be sure, many experts once assured courts that the examination process was so rigorous that no competent examiner meticulously following the prescribed steps could reach a mistaken conclusion. ${ }^{102}$ Today, however, many experts understand that insistence on "perfect accuracy," "no errors," and "a zero error rate" 103 for the ACE-V "method itself, if followed correctly ... does not lead to a process of method improvement"104 and that these claims "have no scientific basis" 105 and "are not scientifically plausible." 106 Commentary from the latent print community "acknowledges that errors do occur and furthermore that claims of zero error rate in the discipline are not scientifically plausible." 107

But if the rates of various kinds of errors are not zero, how can we know what they are, and what use can courts make of the possible estimates? The frequency with which different types of errors occur - and even whether meaningful data exist or can be produced to estimate this frequencyare subject to differing opinions. In a lengthy review, Cole concluded that "the existing data are inadequate to calculate a meaningful error rate for forensic fingerprint identification." 108 Pointing to "known cases of fingerprint misattribution" and a $0.8 \%$ rate of false positives on external proficiency tests, however, he proposed that "the error rate may not be trivial." 109 The NRC committee was more circumspect when it wrote that "there is limited information about the accuracy and reliability of friction ridge analyses." 110 In response, the Scientific Working Group on Friction Ridge Analysis, Study and Technology insisted that

history demonstrates that the actual error rate in practice is very low. ... Billions of comparisons worldwide have occurred over the course of a century with an extremely low number of errors. ... Recent studies published in peer-reviewed journals, although limited, also tend to suggest that the error rate of friction ridge examination, when conducted by competent examiners, is very low. ${ }^{111}$

Likewise, in United States v. Baines, ${ }^{112}$ a former Federal Bureau of Investigation (FBI) supervisor testified that the FBI had "made, on average, about one erroneous identification every 11 years." Estimating the total number of comparisons made at about 1 million per year, he assured the court that the known actual error rate was about one per eleven million comparisons.

\footnotetext{
${ }^{102}$ For examples, see United States v. Baines, 573 F.3d 979, 997-98 (10th Cir. 2009); Government's Combined Report to the Court and Motions in Limine Concerning Fingerprint Evidence, at 22, United States v. Mitchell, 199 F. Supp. 2d 262 (E.D. Pa. 2002), aff'd, 365 F.3d 215 (3d Cir. 2004); United States v. Llera Plaza, Crim. No. 98-362-10, 11, 12, 2002 U.S. Dist. LEXIS 344 (E.D. Pa. Jan. 7, 2002), vacated, 188 F. Supp. 2d 549 (E.D. Pa. 2002).

${ }^{103}$ NAS, NRC, Committee on Identifying the Needs of the Forensic Science Community, op. cit., p. 47.

${ }^{104}$ Ibid., p. 143.

105 Zabell, S. "Fingerprint Evidence.” Journal of Law and Policy, 13 (2005): 177.

${ }^{106}$ NAS, NRC, Committee on Identifying the Needs of the Forensic Science Community, op. cit., p. 143.

${ }^{107}$ Scientific Working Group on Friction Ridge Analysis, Study and Technology. Strengthening Forensic Science in the United States: A Path Forward, Position Statement. Scientific Working Group on Friction Ridge Analysis, Study and Technology, 2009. See also Champod, C. "Fingerprint Examination: Towards More Transparency." Law, Probability \& Risk, 7 (2008): 111.

${ }^{108}$ Cole, S. "More Than Zero: Accounting for Error in Latent Fingerprint Identification." Journal of Criminal Law and Criminology, 95 (2005): 1033.

109 Ibid.

${ }^{110}$ NAS, NRC, Committee on Identifying the Needs of the Forensic Science Community, op. cit., p.143.

${ }^{111}$ SWGFAST, 2009, op. cit.

112573 F.3d 979, 984 (10th Cir. 2009).
} 
But the reliance on denominators of millions and "billions of comparisons" has been challenged on the grounds that

the chances of uncovering an erroneous identification are remote. Most fingerprint identifications are not challenged in court either because the defendant pled to some other charge or because the defense did not obtain a second opinion. Further, after conviction, the opportunities for innocent persons to obtain new evidence and have their convictions reviewed and overturned are still extremely rare. ${ }^{113}$

Historical inquiry is simply not a viable way to estimate how low the false positive rate has been, let alone to estimate the size of the false negative rate.

Instead, one might consider using data from five possible sources: non-blind proficiency tests currently in use; realistic, blind proficiency tests; verifications; random audits of case reports; and controlled experiments. These kinds of data vary in their suitability for estimating error rates, and all of them have significant limitations. To begin with, proficiency tests designed and administered for certification and quality improvement purposes bear little resemblance to actual casework. The employees or trainees taking them are not blind to the fact that they are being tested, and the samples prepared for certification and quality improvement purposes can be different from casework samples. For such reasons, one large proficiency test provider has cautioned against using its proficiency test data for calculating error rates. ${ }^{114}$

Second, recognizing that normal proficiency tests are neither designed for nor particularly suitable for estimating error rates, some commentators have proposed more realistic, blind proficiency testing to produce estimates of error rates for individuals, organizations, or the entire profession. ${ }^{115}$ Truly blind proficiency testing is possible, but implementation on a large scale may not be feasible given the logistics and costs. ${ }^{116}$ For the entire laboratory to be blinded, law enforcement authorities must disguise the test so that it appears as a routine case to the target laboratory. European DNA laboratories undergo two "blind trials" a year with many samples, but these are really "a 'graded' interpretation test." 117 The commission that designed the trials "seriously considered" but rejected "the setting up of a 'fake' or simulated casework situation to be distributed among the participants ... [because] this was deemed to be extremely difficult to implement from a practical point of view and also to offer no great advantage to the testing procedure." 118 Thus, if actual error rates are quite small, an impractical number of realistic proficiency tests (in which the entire laboratory is blind to the testing) would be required to

\footnotetext{
${ }^{113}$ Haber, L. and R. Haber. "Scientific Validation of Fingerprint Evidence Under Daubert.” Law, Probability \& Risk, 7 (2008): 87. See also S. Cole, op. cit.; Saks, M. "Merlin and Solomon: Lessons From the Law's Formative Encounters with Forensic Identification Science.” Hastings Law Journal, 49 (1998): 1102, note 169.

${ }^{114}$ Collaborative Testing Services, Inc. CTS Statement on the Use of Proficiency Testing Data for Error Rate Determination. Collaborative Testing Services, Inc, March 30, 2010.

${ }^{115}$ E.g., Koehler, op. cit.

${ }^{116}$ Peterson, J., G. Lin, M. Ho, et al. "The Feasibility of External Blind DNA Proficiency Testing." Journal of Forensic Sciences, 48 (2003): 21.

${ }^{117}$ Butler, J. Fundamentals of Forensic DNA Typing. Academic Press, 2010, p. 299.

${ }^{118}$ Rand, S., M. Schürenkamp, and B. Brinkmann. "The GEDNAP (German DNA Profiling Group) Blind Trial Concept.” International Journal of Legal Medicine, 116, no. 4 (2002): 199.
} 
produce accurate estimates. ${ }^{119}$ Using the upper bound of a confidence interval based upon a small sample could grossly overstate the likelihood of an error in a particular case (see Box 2.3). ${ }^{120}$

Third, forensic service providers that routinely use verifications could compile statistics on the outcomes. Because verifications are less costly than blind proficiency tests, a much larger number of observations would be available, leading to a more precise estimate. However, at best, statistics on the outcomes of verifications estimate reproducibility rather than validity. That two examiners agree does not prove that both are correct; that they disagree does not tell us which one is correct.

Consequently, statistics on verifications would not estimate false positive or false negative probabilities.

This is not to say that verification statistics have no relevance to error probabilities. If examiners usually reach conflicting results, one can infer that the ability to make correct classifications of pairs of prints is limited. Establishing reproducibility, therefore, is a part of the process of validating measurements, but concordance between the two examiners is a flawed measure even of reproducibility if the verifying examiner's judgments are influenced by knowledge of the first examiner's opinion. Forensic service providers that conduct a large number of blind verifications, however, could contribute to a more complete base of knowledge about the examination process by compiling statistics on these verifications. That verifying examiners, proceeding with no knowledge of the outcome of the initial examination, almost always reach the same conclusions

\footnotetext{
${ }^{119}$ Champod, op. cit., p. 112; National Academy of Sciences, National Research Council, Committee on DNA Forensic Science: An Update. The Evaluation of Forensic DNA Evidence. National Academies Press, 1996.

${ }^{120}$ Ibid.

${ }^{121}$ Hanley, J. and A. Lippman-Hand. "If Nothing Goes Wrong, Is Everything All Right? Interpreting Zero Numerators.” Journal of the American Medical Association, 249 (1983): 1743.

${ }^{122}$ Louis, T. "Confidence Intervals for a Binomial Parameter After Observing No Successes." The American Statistician, 35 (1981): 154; Hanley, op. cit.

${ }^{123}$ Bickel, P. and K. Doksum. Mathematical Statistics. Holden-Day, 1977.

${ }^{124}$ Winkler, R., J. Smith, and D. Fryback. "The Role of Informative Priors in Zero-Numerator Problems: Being Conservative Versus Being Candid.” The American Statistician, 56 (2002): 1.
} 
as initial examiners would indicate that the judgments of different examiners are at least reproducible. Moreover, whether or not the verifications are blind, the data on initial discrepancies could be helpful in identifying individual examiners or situations that merit constructive, corrective action.

Fourth, the outcomes of random audits of completed casework might be used to estimate false positive or false negative probabilities. For quality assurance, random technical case audits test the entire scope of evidence examination and reporting. Such audits can be conducted internally (by the same agency that did the examination) or externally (by an outside entity). These technical case audits include a re-analysis of the evidence and an associated evaluation. As with proficiency tests, the purpose of conducting technical case audits is to improve laboratory performance and to increase reliability and reproducibility. There is an inherent value of a program including these audits (regardless of any particular case audit outcomes) that results simply from each examiner knowing that some of his work will be re-examined. This knowledge may make people more conscientious in their documentation and performance.

Case audits are designed to detect deviations from ideal, normative, or intended practices. Finding these alone makes the audit process useful. The findings can identify specific corrective actions (as they relate to the case), or they can identify more general issues, resulting in recommendations for improvements (for example, changes in processes or in training methods). Audits generally do result in such findings, since all processes can be improved; arguably, if an agency performs audits, it should make them rigorous enough for the agency to acquire data on areas for improvement. Thus, compilations of technical case audit findings are a potential source of valuable information. They provide quantitative data regarding the occurrence of different types of deviations from ideal or intended practices. The data can suggest which deviations may be occurring systematically, leading to modifications in technical audit procedures or to more specific investigations or research.

Technical case audit compilations, however, are not a reasonable way to measure overall latent print process performance or to estimate the error rate of a specific latent print process. Individual technical audits differ in their criteria, rigor, and areas of concern (as is appropriate and consistent with their purpose). Compilations of findings will represent combinations of cases with different examiners, different levels of difficulty, and different examination processes. The actual causes of problems detected in technical case audits usually cannot be determined. And, once again, ground truth, which would be necessary for establishing errors in identifications and exclusions, is unknown for audited casework.

Rather than auditing casework, an expert panel of latent print examiners could conduct its own evaluations of a large sample of previous casework (blinded to the earlier outcomes). ${ }^{125}$ The results would test the conclusions reached in actual casework. However, the true source of the latent prints in these cases would not be known with certainty. The panel's conclusions would have to be accepted as correct if they are to serve as the measure of accuracy in casework. Without proof of the panel's accuracy, the experiment would be subject to the criticism that it seeks to prove one unknown by means of another. Although the absence of ground truth technically makes this experiment a reproducibility study, the expert panel experiment could be

${ }^{125}$ See SWGFAST, 2009, op. cit. 
revealing. Studies of the predictive power of screening tests in medicine rely on this experimental design when they use a more precise (but still imperfect) test to measure the accuracy of the first result. A large number of discordant evaluations would reveal the need for further research targeted to ascertaining the sources of the discrepancies. Conversely, high concordance rates would support the perception that latent print analysis is a reliable and reproducible process as currently practiced.

Finally, controlled experiments with simulated materials are possible. These could illuminate the conditions under which possible errors might occur, could help provide a scientific foundation for the validity of the ACE-V process (as practiced by the subjects in the experiment), and could supply the kind of error rate information mentioned in Daubert v. Merrell Dow Pharmaceuticals, Inc. ${ }^{126}$ To determine error rates of a specific type, or for a specific process, one can design an experiment that has a consistent process and for which the correct outcomes are known. In this way, an error can be detected conclusively (as distinct from a process divergence examined in case audits).

This is the logic that underlies the European collaborative exercises in the DNA field. ${ }^{127}$ The researchers conducting these exercises have found that the overall error rate dropped sharply with the introduction of standardized, commercially available DNA testing kits in 1999; that "human carelessness is the predominant source of error regardless of the technology used;" that these errors take the form of "the transposition of samples, ... albeit never [twice] by the same laboratory" and "writing a correct result incorrectly in the summary sheet supplied" but not normally used in the laboratory; ${ }^{128}$ and that the total error rate has stabilized in the range of $0.4 \%$ to $0.7 \% .{ }^{129}$ The researchers' conclusions indicate the value of experiments like these: "During the development of the trials over the last decade since the introduction of STRs [short tandem repeats], many lessons have been learnt which have led to improvements not only in the trials themselves, but also have achieved the goal of improved quality of results within the forensic community as a whole." 130

Results from the first large-scale, controlled experiment on the accuracy of fingerprint examiners appeared in 2011. ${ }^{131}$ Sacrificing the realism of casework for known ground truth, experimenters from the Noblis Corporation and the FBI presented latent print examiners with pairs of prints consisting of one latent print and one exemplar, each from known sources. Some of the pairs (520) were mates that came from the same finger. The rest (224) were nonmates that came from different fingers. These nonmated pairs were designed to yield difficult comparisons. Unusually similar exemplars in the nonmates came from searches of the prints of the 58 million persons then in FBI's Integrated AFIS database. Moreover, an abnormally large proportion of the latent prints were of poor quality.

\footnotetext{
126509 U.S. 594 (1993).

${ }^{127}$ Rand, Schürenkamp, and Brinkman, 2002, op. cit.

${ }^{128}$ Ibid.

${ }^{129}$ Rand, S., M. Schürenkamp, and B. Brinkmann. "The GEDNAP (German DNA Profiling Group) Blind Trial Concept Part II: Trends and Developments." International Journal of Legal Medicine, 118, no.2 (2004): 83.

${ }^{130}$ Ibid., p. 88.

${ }^{131}$ Ulery, B., A. Hicklin, J. Buscaglia, et al. "Accuracy and Reliability of Forensic Latent Fingerprint Decisions." Proceedings of the NAS. 2011.
} 
After a double-blind presentation of the mated and nonmated pairs to the latent print examiners, the experimenters measured the proportions of identifications ${ }^{132}$ for the mates (sensitivity) and nonmates (false positives), as well as the proportions of exclusions for the nonmates (specificity) and mates (false negatives). For the particular mix of mates and nonmates in the test set, they also determined the proportion of correct judgments among the identifications (positive predictive value) and exclusions (negative predictive value). Box 2.4 provides a small part of the data obtained and the resulting statistics on errors and accuracy.

Of course, the experimental results may not lead to reasonable estimates of the rates at which errors occur in practice. To the extent that the experimental conditions and the examiners who are tested in the experiment do not mirror the conditions and examiners encountered in actual casework, the measured error probabilities will not apply directly to the field as a whole. They may, however, answer such questions as "How well do skilled examiners, working under ideal conditions on a difficult case, perform?" As noted, the legal system values the answers to such questions. Chapter 6 considers the admissibility of various statistics on error rates and offers recommendations on how an expert witness might approach the issue of error in a particular case.

\section{Box 2.4: Selected Results of the Noblis-FBI Experiment}

This experiment presented 169 relatively experienced and proficient latent fingerprint examiners, who knew they were being tested, with pairs of latent and exemplar prints selected to be challenging and judged by the examiners in the study to be representative of casework. The examiners worked through a total of 17,121 presentations of 744 image pairs (roughly 100 pairs per examiner). Table 2.8 summarizes the results of the examiners' efforts for those pairs of prints that they initially deemed "of value for individualization." 133

\begin{tabular}{|r|r|r|r|}
\cline { 2 - 4 } \multicolumn{1}{c|}{} & \multicolumn{1}{c|}{ Nonmate } & \multicolumn{1}{c|}{ Mate } & \multicolumn{1}{c|}{ All } \\
\hline Exclusion & 3,622 & 450 & 4,072 \\
\hline Identification & 6 & 3,663 & 3,669 \\
\hline Inconclusive & 455 & 1,856 & 2,311 \\
\hline All & 4,083 & 5,969 & 10,052 \\
\hline
\end{tabular}

Table 2.8: Outcomes for pairs judged to be "of value for individualization"

Of the presentations deemed of value for individualization $(59 \%$, or 10,052$)$, five examiners $(5 / 169=3 \%)$ made false identifications. One of the five examiners made two false identifications. Looking just to the outcomes likely to be seen in court-the identifications and exclusions - the false positive rate is $6 / 3,628=0.2 \% .{ }^{134}$ (For brevity, the discussion here is

${ }^{132}$ During the study, examiners were asked to provide one of four decisions: "the analysis decision of no value (unsuitable for comparison); or the comparison/evaluation decisions of individualizations (from the same source), exclusion (from different sources), or inconclusive." Ibid.

${ }^{133}$ There were an additional 3,122 comparisons based on latent fingerprints deemed of value only for exclusion. Because standard operating procedures typically include only value-for-individualization comparisons, these other outcomes are not presented here.

${ }^{134}$ In no case did two examiners make the same false positive error. The errors occurred on image pairs where a large majority of examiners made correct exclusions; one occurred on a pair where the majority of examiners judged the comparisons to be inconclusive. Thus, the six erroneous identifications probably would have been detected if an independent, blind verification were performed as part of the operational examination process. 
confined to this set of cases in which the examiners were able to make an identification or an

exclusion. ${ }^{135}$ )

As discussed earlier, "sensitivity" is the proportion of cases in which an examiner confronted with a mated pair makes a positive identification. Thus, in this experiment, the sensitivity was $3,663 / 4,113=89.1 \% .{ }^{136}$

Whereas the false positive rate was only $0.2 \%$, the false negative rate was $450 / 4,113=10.9 \% .{ }^{137}$ Defining specificity as the proportion of exclusions for nonmates judged to be of value for inclusion, the specificity was $3,622 / 3,628=99.8 \% .{ }^{138}$ Table 2.9 summarizes these error and accuracy rates.

\begin{tabular}{|r|l|l|}
\cline { 2 - 3 } \multicolumn{1}{c|}{} & \multicolumn{1}{c|}{ Nonmate } & \multicolumn{1}{c|}{ Mate } \\
\hline Exclusion & Specificity: $99.8 \%$ & False negative rate: $10.9 \%$ \\
\hline Identification & False positive rate: $0.2 \%$ & Sensitivity: $89.1 \%$ \\
\hline
\end{tabular}

Table 2.9: Accuracy and error rates for exclusions and identifications in pairs judged to be "of value for individualization" and leading to exclusions or identifications

\subsection{Summary}

This chapter discussed types of error and the differences between process errors and outcome errors. It also reviewed how the system in which the examiner works can contribute to such errors. Finally, the chapter outlined the challenges and benefits of trying to calculate the prevalence of error in latent print examinations.

\footnotetext{
${ }^{135}$ Adding inconclusives lowers the false positive rate to $6 / 4,083=0.1 \%$.

${ }^{136}$ Again, the text deals only with the pairs deemed of value for identification and resulting in a definitive conclusion. The sensitivity was 3,663/5,969 $=61.4 \%$, including inconclusives.

${ }^{137}$ Adding inconclusives lowers the false negative rate to $450 / 5,969=7.5 \%$.

${ }^{138}$ Adding inconclusives lowers the specificity to $3,622 / 4,083=88.7 \%$.
} 


\section{Chapter 3: Interpreting Latent Prints}

\section{Introduction and Scope}

Latent print examination requires the careful interpretation and comparison of friction ridge impressions. From the first examination of the features contained in a latent print to a final conclusion regarding an identification or exclusion, an examiner engages in many acts of interpretation. Current methods of interpretation are based upon professional knowledge and experience rather than formal decision thresholds or statistical models.

This chapter describes the critical interpretative stages that are part of every latent print comparison. It surveys the current state of knowledge, based upon published research, regarding the interpretive stages and the implications for pitfalls that may arise during the interpretive process. It also identifies the most important questions in this area that require further study. Finally, this chapter provides recommendations and best practices relating to the interpretive stages.

\subsection{Preliminary Observations and Caveats}

Several preliminary observations are important. To begin with, the chapter focuses almost exclusively on latent fingerprint examinations using the ACE-V process (Analysis, Comparison, Evaluation, and Verification), drawing on the Latent Print Examination Process Map of Chapter 1 (Figure 1.1). This chapter maps the interpretive stages onto the relevant sections of that Process Map. The focus on ACE-V is not intended as an endorsement of ACE-V as a "methodology." As explained in Chapter 1, ACE-V maps the steps of a process, but it does not provide specific functional guidance on how to implement that process, nor does it detail the substantive content of the various steps. ${ }^{139}$ Although ACE-V provides a useful framework for describing the steps taken for interpreting prints, it does not offer specific criteria to guide those interpretations.

A second preliminary observation concerns the analysis of the subjective elements in human interpretation. To recognize such subjectivity is not to disparage the process. The act of interpreting inevitably requires human judgment and necessarily implies at least some subjectivity. If one simply follows a clear, bright-line rule with no discretion or independent judgment, one is not actually interpreting. Interpretation goes beyond merely executing a set of rules; thus, the exercise of judgment and expertise inevitably makes the interpretive process partly subjective.

Third, with the subjectivity of interpretation comes the possibility of reduced performance because of human factors issues. As explained in Chapter 1, cognitive bias is one aspect of human interpretation that has received significant attention. Writing about sorting fingerprints according to Purkenje's standards, Francis Galton recognized the difficulty of accurate interpretations of fingerprints and the possible dangers of cognitive bias:

${ }^{139}$ See also Mnookin, J. "The Courts, the NAS, and the Future of Forensic Science." Brooklyn Law Review, 75, no. 4 (2010): 1209. 
On considering the causes of these doubts and blunders, different influences were found to produce them, any one of which was sufficient by itself to give rise to serious uncertainty. A complex pattern is capable of suggesting various readings, as the figuring on a wallpaper may suggest a variety of forms and faces to those who have such fancies. ${ }^{140}$

All human interpretation is potentially subject to biasing effects, as the enormous research literature across a wide variety of domains establishes (as noted in Chapter 2). To recognize that latent print examiners are potentially subject to bias is not to single them out but rather to suggest that they are not exempt from those cognitive biases that all interpreters of data and information face. Indeed, many psychologists claim that susceptibility to bias is part of the architecture of the human brain. The same processes that help us make sound judgments in many circumstances also create the possibility for bias. In some instances, cognitive biases lead to improved performance, whereas in other circumstances they degrade performance. ${ }^{141}$

Bias is only one of the many factors that can affect examiner performance. It receives particular attention in this chapter because of the extensive research on cognitive bias in other fields and the emerging research in this field that reveals the possible effects of bias on interpretive performance. Other chapters focus on other factors - from lighting, to technology access, to work culture - that also affect interpretation. Recognizing that a variety of human factors affect the interpretive process, this chapter examines the process with an eye toward identifying the facets that raise research and operational issues because of the possibility of cognitive bias.

Fourth, although this chapter attempts to highlight those locations within the interpretive process of latent print examination where subjectivity and error due to human factors might be especially salient, it makes no effort to quantify the potential risk of error in latent print interpretation. The discussion is primarily theoretical. For the most part, the empirical basis for clearly establishing the extent of these risks does not yet exist. Further research is needed to establish whether, in what circumstances, and to what extent these theoretical issues of interpretation create an elevated risk for error.

Many of the interpretive issues discussed in this chapter are well known to latent print examiners. However, some of the interpretive inflection points in the latent print identification process traditionally have not had precise names associated with them and are not clearly articulated in day-to-day practice. Enormous variability exists in how various forensic service providers approach these interpretive issues. This chapter offers a framework for thinking about interpretation, and it presents a vocabulary for considering the interpretive inflection points, but it does not attempt to detail the variations in approaches to interpretive questions across laboratories or jurisdictions. The major interpretive stages involved in latent print examination discussed in this chapter are as follows:

- Feature selection: The examiner selects specific friction ridge details within a latent print on which to focus subsequent inquiry.

\footnotetext{
${ }^{140}$ Galton, F. Finger Prints. 1892. Reprint, William S. Hein \& Co., 2003, p. 66.

${ }^{141}$ Potchen, E., J. Gard, P. Lazar, et al. "The Effect of Clinical History Data on Chest Film Interpretation: Direction or Distraction?” Investigative Radiology, 14 (1979): 404
} 
- Utility assessment: At various points in the latent print identification process, an examiner decides whether the print is of value for numerous purposes. Examples include assessing whether the latent is suitable for comparison, whether it is suitable for an automated fingerprint identification system (AFIS) search, and whether the potential source print is suitable for comparison. Because these interpretive determinations are all forms of sufficiency analysis, they are considered under a common rubric.

- Feature weighting: An examiner assigns weight and significance to individual features and their configuration and assesses the overall strength of their synthesis.

- Discrepancy interpretation: An examiner interprets the significance of observed divergences between a latent print and an exemplar and determines whether the differences establish that the two impressions arose from different sources or are consistent with a common origin.

- Source attribution: An examiner decides whether two prints share a common source, or determines that the information presented does not permit a categorical conclusion. ${ }^{142}$

In addition to discussing these interpretive stages individually, this chapter addresses a number of issues common to several interpretative stages, including the need to establish adequate documentation and the desirability of procedures to help avoid bias. The next section discusses these crosscutting issues and presents recommendations regarding them. The remainder of the chapter (1) describes the interpretive stages and explicitly connects them to the Latent Print Examination Process Map set out in Chapter 1, (2) describes and discusses any key published research findings that relate to the particular interpretive stage, (3) provides recommendations and best practices relating to the interpretive stage, and (4) highlights important research questions connected to each interpretive stage.

\subsection{Issues Relevant to Multiple Interpretative Stages}

\subsubsection{Documentation}

One crosscutting issue is the need for adequate documentation of an examiner's reasoning and conclusions. Documentation is not itself an interpretive practice, but rather a practice for capturing an examiner's interpretive judgments in a form that would permit the examiner, or another examiner, to make sense of a decision at a later time. Documentation serves to maximize the transparency of the interpretative process and to provide a record that can be useful for many purposes, including reports and testimony, future research and evaluation, and quality assurance. Documentation requirements also may affect the interpretive process itself by requiring judgments to be explicit and thus potentially subject to greater reflection on the part of the examiner.

142 There are other interpretive stages beyond the five described here. For example, it can be argued that a major step is classifying an impression as emanating from a finger or palm and determining what orientation to use to search the latent print. These decisions, if incorrect, can lead to a missed opportunity for an identification or to an erroneous exclusion. However, the focus here is on the steps that can present some of the more complex issues in comprehending the associated human factors concerns. 
Questions regarding how much documentation should be required clearly are relevant to feature selection, utility assessment, and discrepancy interpretation. How much documentation to provide is difficult to legislate because the degree and detail of documentation can vary depending on the complexity of the comparison. The more complex the cognitive and interpretive assessment, the more there is to be gained from detailed and explicit documentation. However, assessing the complexity of a comparison requires additional research. Without clear guidelines on complexity, the examiner's subjective assessment of complexity can be used, but this assessment too should be part of the record. For both legal and scientific purposes (see Chapter 5), the documentation should supply detail sufficient for another expert to understand the basis for the examiner's conclusions and to replicate the steps the examiner followed.

Such documentation should be contemporaneous with the analysis or as nearly so as is practicable. ${ }^{143}$ Because the purpose of documentation is to capture an examiner's interpretive process, both to assist that examiner in recalling what occurred and to make these processes as transparent as possible to others, documentation will be most helpful and accurate when conducted at the same time as the interpretation itself. ${ }^{144}$ The closer in time the documentation is conducted to the mental processes it details, the more complete and accurate the documentation is likely to be.

\section{Recommendation 3.1: A report and contemporaneous supporting notes or materials should document the examination to make the interpretive process as transparent as possible. Although the degree of detail may vary depending on the perceived complexity of the comparison, documentation should, at a minimum, be sufficient to permit another examiner to assess the accuracy and validity of the initial examiner's assessment of the evidence.}

Another question that arises in more than one interpretative stage is the extent to which it is legitimate to reassess a latent print or other exemplar after looking at a potential source print. Norms and laboratory practices vary in this regard. At one extreme, some forensic service providers permit examiners to undertake comparisons without any prior explicit feature assessment in the questioned print. At another extreme, some providers discourage any "new" features from being noted in the latent print after comparison has begun. Anecdotal evidence suggests that many laboratories routinely reassess the features within the latent print after undertaking a comparison to a known exemplar. This recursive practice should not be ruled out, because the exemplar may draw attention to a genuine feature in the latent print that was not previously observed or that was interpreted as likely to be an artifact until the same feature was apparent in the comparison print. ${ }^{145}$

However, recursion raises the possibility that an examiner will be unduly influenced by the exemplar when reassessing the latent print. In other words, although the comparison process may reveal true similarities regarding features that had not previously been noted, it also can lead to

\footnotetext{
${ }^{143}$ See American Society of Crime Laboratory Directors/Laboratory Accreditation Board. ASCLD/LAB Guiding Principles of Professional Responsibility for Crime Laboratories and Forensic Scientists. Principle 15, Version 1.1. ${ }^{144}$ See U.S. Department of Justice, Office of the Inspector General. The FBI DNA Laboratory: A Review of Protocol and Practice Vulnerabilities. U.S. Department of Justice, May 2004.

${ }^{145}$ Vanderkolk, J. Forensic Comparative Science: Qualitative Quantitative Source Determination of Unique Impressions, Images, and Objects. Academic Press, 2009, p. 23, 92 - 94.
} 
the perception of a similarity that does not exist. In some instances, the comparison print may help the examiner accurately clarify ambiguous minutiae in the latent image, whereas in other instances, an examiner may, in all good faith, be misled by what cognitive psychologists call expectation effects. Given this complex interaction between the interpretation of a latent and known print, this report can offer no hard rules about the appropriateness of making use of this interpretive practice. However, procedural steps can make the use of this interpretive procedure more transparent and reflective. At a minimum, there should be an explicit determination of features in the latent before the comparison process. This initial determination need not limit the features that can be used in subsequent analysis; identifying this initial set ensures that the analyst's approach remains transparent. But because of the danger of bias emerging in going back and forth between latent and exemplar prints - and to maintain the transparency of the process - any features that are noted after comparison has begun or as the result of the comparison process (rather than before comparison begins) should be indicated and explicitly included in the documentation.

\section{Recommendation 3.2: Modifications to the results of any stage of latent print analysis (e.g., feature selection, utility assessment, discrepancy interpretation) after seeing a known exemplar should be viewed with caution. Such modifications should be specifically documented as having occurred after comparison had begun.}

\subsubsection{Cognitive Biases}

Another crosscutting issue is cognitive bias. Chapter 1 notes the substantial body of psychological research illustrating the tendency for human beings to engage in motivated reasoning or to be affected by contextual knowledge. Even people attempting to be fair interpreters of the evidence may be influenced by information outside the formally relevant decision criteria. Such biases may be present in the work of latent print examiners. ${ }^{146}$ However, the extent to which cognitive bias creates the danger of erroneous interpretations in real-world circumstances has not yet received significant research attention. Continued research about the presence or absence of such biases in the latent print domain and the extent of any impact on accuracy and reliability is needed. However, given the decades-long research into the significant effects of cognitive bias in other domains, it seems wise to minimize the potential for such biases in latent print interpretation, even in the absence of definitive research results for latent print analysis.

An obvious way to minimize the effect of contextual information is to keep the interpreter from having access to that contextual information in the first place. For example, the double-blind study is the gold standard for clinical drug testing because it ensures that the clinicians administering a drug and evaluating the patient's response do not know whether the patient is receiving the drug or the placebo. Treating physicians still have all the information they need to provide quality care, but those participating in the testing process are shielded from the information that could bias them.

${ }^{146}$ Dror, I., D. Charlton, and A. Peron. "Contextual Information Renders Experts Vulnerable to Making Erroneous Identifications.” Forensic Science International, 156, no. 1 (2006): 74-78. See also authorities cited, Chapter 2. 
Currently, there is no systematic effort to blind forensic examiners from contextual information that is clearly not domain relevant - information that is not related to or necessary for their analysis. For example, the suspect's prior convictions, gang membership, or confession does not assist in the print analysis. Such extraneous information could create bias. It is therefore good practice to avoid disclosing extraneous, domain-irrelevant information. ${ }^{147}$ To be sure, someinformation may be necessary at a later point in the forensic analysis and should be provided at that point. The goal here is not to define what information is necessary at each point in the analysis but simply to suggest that procedures should be designed to eliminate access to potentially biasing, domain-irrelevant information.

\section{Recommendation 3.3: Procedures should be implemented to protect examiners from exposure to extraneous (domain-irrelevant) information in a case.}

This recommendation is not suggesting that examiners should be denied access to information that is legitimately relevant to their substantive analysis. Examiners must have the information that is necessary to do their jobs effectively. Some information will be both potentially biasing and domain relevant - for example, information about the substrate from which a print was lifted, or the fact that the source print is the result of an AFIS search. Unless further research points to particularized dangers of bias, examiners should have access to whatever information is legitimately relevant to the exercise of their expertise, notwithstanding some theoretical but unproven danger of bias.

However, given the genuine dangers of cognitive bias, the better practice is to protect examiners from inadvertent bias by shielding them from information that is clearly unnecessary and not relevant to their assessment. It might not always be feasible to implement such procedures, and it may well be infeasible to implement them perfectly. Forensic scientists strive to reach conclusions "based on the evidence and reference material relevant to the evidence, not on extraneous information, political pressure, or other outside influences." 148 If examiners routinely receive extraneous, domain-irrelevant information, forensic service providers should examine whether they can modify their procedures to shield examiners from this unnecessary and potentially biasing information.

The three recommendations above apply to multiple interpretative stages. The sections that follow focus on the stages of the latent print analysis, with reference to the research that is relevant to each stage and recommendations (beyond those given above) that are specific to each stage of interpretation.

\subsection{Feature Selection}

The first major interpretative stage in a latent print analysis occurs during the initial examination of the latent print. In this stage, the examiner identifies features in the latent print (e.g., ridge

\footnotetext{
${ }^{147}$ Others have made similar suggestions. E.g., Risinger, D., M. Saks, W. Thompson, et al. "The Daubert/Kumho Implications of Observer Effects in Forensic Science: Hidden Problems of Expectation and Suggestion." California Law Review, 90, no. 1 (January 2002); Krane, D., S. Ford, J. Gilder, et al. "Sequential Unmasking: A Means of Minimizing Observer Effects in Forensic DNA Interpretation.” Journal of Forensic Sciences, 53, no. 4 (2008): 1006-7.

${ }^{148}$ ASCLD/LAB, Principle 2, op. cit.
} 
flow type or ridge path minutiae) that allow a determination of whether the latent print has sufficient detail to form the basis for subsequent comparisons. Section 3.5 provides figures and more explanation about minutiae and the levels of detail in print images.

\subsubsection{Corresponding Phase in the Latent Print Examination Process Map}

One of the primary tasks of the Analysis phase of the ACE-V process is the selection of reliable friction ridge features in a latent print for comparison against features in exemplar prints. This corresponds to steps 210 and 320 in the Latent Print Examination Process Map (Figure 1.1). The process of feature selection may occur simultaneously with the other interpretative processes such as analysis of distortion and suitability determination (see the discussion of these topics below and steps 210 through 240 in the Process Map). These interpretative processes, in fact, may all color and influence each other because they have direct impact on the determination of the quantity, quality, and reliability of the features selected for analysis.

\subsubsection{Findings from Existing Research}

Feature selection begins with an analysis of the latent print by itself. This generally occurs - and should generally occur-before viewing the exemplar print. The features that will be selected usually include those that are the most clearly visible and distinctive, and they will serve as the basis for the comparison against exemplar prints.

In studying a latent print, an examiner will likely note some minutiae that he is certain exist and other that he is less sure about. The former should be the primary focus. Confidence about a feature refers to two things: (1) the examiner is confident that the feature genuinely exists in the latent print, and (2) the examiner strongly expects to observe that feature in a corresponding exemplar print from the same source. The degree of expectation relates to the level of tolerance that the examiner is willing to accept when observing differences during the subsequent Comparison phase. In other words, if an examiner believes a feature exists in the latent print and strongly expects to see it in any print from the same source, then the absence of the feature could significantly affect the examiner's source attribution. The effect of this feature's absence increases with the examiner's confidence that the feature existed in the original latent print (and could not be interpreted as anything else).

Typically, high-confidence features will have the highest reliability (accuracy and precision in selection). ${ }^{149}$ Because these features will form the basis for the decision, the manner in which they are selected, how they are documented, and what level of confidence is assigned to them become critical in understanding the examiner's decision-making process. Feature selection becomes crucial in the examination because criteria for the decision-making processes are neither clearly defined a priori nor made using an objective, validated metric.

\section{Defining the Features}

Examiners are not limited to the "points" or "minutiae" when selecting features during the Analysis phase (although commonly these may be the only formal features documented).

\footnotetext{
${ }^{149}$ Langenburg, G. and C. Champod. "The GYRO System-A Recommended Approach to More Transparent Documentation." Journal of Forensic Identification, 61, issue 4 (July/August 2011): 373 - 384.
} 
Examiners will utilize several classes of features, often referred to as "levels of detail." There are also occasional features not associated with any of the traditional levels of detail that appear, such as creases and wrinkles, permanent scars, temporary skin damage, or skin disease, that examiners might use. ${ }^{150}$

Level 1 Detail (L1D) is defined as "ridge flow." 151 Ridge flow often translates to a pattern type in a finger or palm, such as a loop, whorl, or arch formation; ridge flow also includes other information such as relative curvature. Pattern types are class characteristics shared by many individuals. Furthermore, L1D can be easily distorted as a result of the flexibility of skin. ${ }^{152}$ Therefore, although L1D might be selected, this level of detail is not always reliable, especially if signs of distortion appear in the image. Figure 3.1 provides illustrations of some L1D.

Level 2 Detail (L2D) is defined as "ridge path." ${ }^{53}$ L2D includes minutiae, such as ridge endings, bifurcations, or dots. Even the absence of minutiae in an area (called an "open field") can be significant and highly discriminating. Ridge path features are also some of the most robust features, and they will be present even under extreme conditions of distortion. ${ }^{154}$ They also tend to be highly discriminating ${ }^{155}$ and thus are ideal features for comparisons.

Level 3 Detail (L3D) refers to the edges of ridges (which may appear indented or protruded) and pores (the location of the center of the pore, not the size or shape, which can be highly variable within a source). ${ }^{156}$ Although the reproducibility of pores in latent prints (or low-resolution capture systems for exemplars) can be problematic in low-clarity images, these features can be highly discriminating when visible. ${ }^{157}$

\footnotetext{
150 This will vary significantly among jurisdictions. For example, in countries that require a minimum number of minutiae to declare an identification, scars and creases may not be used to reach the minimum. Other agencies may be unwilling to use creases to contribute to the identification decision but will use creases to narrow a search through set of exemplar prints.

${ }^{151}$ Ashbaugh, D. Quantitative-Qualitative Friction Ridge Analysis: An Introduction to Basic and Advanced Ridgeology. CRC Press, 1999.

${ }^{152}$ Maceo, A. "Qualitative Assessment of Skin Deformation: A Pilot Study." Journal of Forensic Identification, 59, no. 4 (2009): 390-440.

${ }^{153}$ Ashbaugh, op. cit.

${ }_{154}$ Maceo, op. cit.

${ }^{155}$ Egli, N., C. Champod, and P. Margot. "Evidence Evaluation in Fingerprint Comparison and Automated Fingerprint Identification Systems - Modelling Within Finger Variability." Forensic Science International, 167 (2007): 189-95; Jain, A., Y. Chen, and M. Demirkus. "Pores and Ridges: High-Resolution Fingerprint Matching Using Level 3 Features.” IEEE Transactions on Pattern Analysis and Machine Intelligence, 29, no. 1 (2007): 15-27; Neumann, C., C. Champod, R. Puch-Solis, et al. "Computation of Likelihood Ratios in Fingerprint Identification for Configurations of Any Number of Minutiae.” Journal of Forensic Sciences, 52, no. 1 (2007): 54-64; Srihari, S., H. Srinivasan, and G. Fang. "Discriminability of Fingerprints of Twins.” Journal of Forensic Identification, 58, no. 1 (2008): 109-27.

${ }^{156}$ Roddy, A. and J. Stosz. "Fingerprint Features-Statistical Analysis and System Performance Estimates." Proceedings of the IEEE, 85, no. 9 (1997): 1390-1421; Richmond, S. Do Fingerprint Ridges and Characteristics Within Ridges Change with Pressure? Australian Federal Police, Forensic Services, 2004.

157 Jain, Chen, and Demirkus, op. cit.; Kryszczuk, K., A. Drygajlo, and P. Morier. "Extraction of Level 2 and Level 3 Features for Fragmentary Fingerprint Comparison." In Proceedings of the Second COST Action 275 Workshop, 2004, 83-88.
} 
Occasional features such as scars and creases can be selected. These features vary in their reproducibility. For example, creases may not always reproduce consistently ${ }^{158}$ but can be fairly discriminating, ${ }^{159}$ whereas scars reproduce very consistently and are also believed to be highly discriminating. ${ }^{160}$ In some instances, such as temporary skin damage, there is a low expectation of reproducibility in exemplar prints, but the presence of such a feature can be highly significant.

How examiners select and document features can vary significantly among jurisdictions. In some instances, the examiner observes and notes features to use for comparison without producing any formal documentation; this examiner might change his mind about the features selected without documenting the change. On the other end of the spectrum, some examiners formally select features a priori and annotate the features during the Analysis phase. In some agencies, examiners must justify in writing any modifications made to their initial feature selections during the Comparison phase. ${ }^{161}$

Whether features are selected formally or informally, there are two major decision components involved: determining the existence of a feature and identifying the type or shape of the feature. These decisions are distinct, and each decision can have its own assigned level of confidence. In other words, deciding if a feature is present is different from determining precisely what the feature is. Both decisions may be limited by the quality and clarity of the image.

The determination that a feature exists is akin to the concept of signal-to-noise ratio determination. Typically, a targeted feature produces a stimulus signal to the observer greater than the background noise. In some instances, additional information near the suspected signal is relevant to ascertaining the existence of a feature. An example would be the observation that adjacent ridges next to a suspected feature react to account for the presence of the suspected feature. Another example would be three ridges entering a small smudged area from which only two ridges exit. These indicators allow an examiner to infer the existence of a feature that cannot be observed directly.

The identification of the type of feature observed is, as a logical matter, a separate inquiry made after determining the existence of a feature. For this decision, the examiner will attempt to classify and identify the type of feature with as much specificity as possible. For example, after noting L1D that includes a recurve and a single delta formation, the examiner might classify this ridge flow as a loop pattern. Similarly, an examiner observing a ridge path event may conclude that the feature is an ending ridge or a bifurcation (illustrations of these minutiae are available in Table 3.4 and Figure 4.3). Or perhaps the examiner cannot tell and will simply refer to the minutiae as a "ridge event." In this last scenario, the examiner has no doubt that a minutia exists in the latent print, but the examiner cannot, on the basis of what is observable in the latent image,

\footnotetext{
${ }^{158}$ Richmond, op. cit.

159 Tietze, S. and K. Witthuhn. Papillarleistenstruktur der menschlichen Handinnenfläche. Luchterhand, 2001.

${ }^{160}$ Feng, J., A. Jain, and A. Ross. "Detecting Altered Fingerprints." In 2010 20th International Conference on Pattern Recognition (ICPR), August 2010, 1622-25.

${ }^{161}$ See, e.g., Interpol European Expert Group on Fingerprint Identification - IEEGFI II. Method for Fingerprint Identification. Interpol European Expert Group on Fingerprint Identification - IEEGFI II, 2004; U.S. Department of Justice, Office of the Inspector General. A Review of the FBI's Handling of the Brandon Mayfield Case (Unclassified and Redacted). U.S. Department of Justice, March 2006.
} 
determine the specific type of minutia. The examiner, in this instance, has great certainty about the minutia's existence, but great uncertainty about its specific nature.

For each of these decisions - determining the existence of the feature and determining the type of feature - the examiner has some internal level of confidence. This level of confidence may be documented formally, or it may simply be an informal or even implicit judgment by the examiner, depending on local practices. The levels of confidence can also be very different for the two decisions. For example, the examiner might be highly confident that a feature exists when three ridges enter a smudged area but only two exit; yet the examiner might have no confidence regarding whether the event is a bifurcation or ridge ending (although the examiner might have high confidence about what the feature is not-for example, a dot).

\subsubsection{Issues and Recommendations}

\section{Confidence Levels and Documentation}

During feature selection, there may be a wholly implicit or a more explicit assignment of weight to the features, mostly as a function of their specificity (discriminating strength). This chapter covers weighting of features in section 3.5; here the focus is on the role of an examiner's confidence in such weighting and the importance of documenting the degree of confidence. An examiner's confidence in the existence of the feature and confidence in its classification will undoubtedly affect the weight applied to the feature, independent of specificity. Generally speaking, examiners would give more weight to a rare feature, such as a trifurcation, when it is clear and distinct and when the examiner is confident in selecting it than when it is part of an indistinct, distorted, or low-quality area that may possibly include a trifurcation or something else (or may not even exist). Weight is a function of both confidence about the feature's existence and the perceived rarity of the feature. Because not enough is known about rarity, and even that which is known is not necessarily part of an examiner's formal training, this aspect of interpretation is often an implicit judgment based on the examiner's experience. ${ }^{162}$

The examiner's decisions in the Analysis phase and the associated levels of confidence will only be captured by documenting these aspects contemporaneously. An examiner may choose to document the features and levels of confidence using a narrative, although this can be somewhat time-consuming and burdensome. Another way to record this information is to annotate the features with dots, tracings, and other markings on a photograph of the latent print. Numerous software packages can facilitate this process. Some software has been designed for fingerprint case annotation and includes tools for marking various friction ridge features, classifying these features, and assigning levels of confidence to the decisions. ${ }^{163}$ Newly developed algorithms, such as used in an automated quality mapping assessment, ${ }^{164}$ may be able to extract features automatically and to assign a level of confidence in the existence of the feature. While waiting

\footnotetext{
${ }^{162}$ Osterburg, J. "An Inquiry into the Nature of Proof. The Identity of Fingerprints.” Journal of Forensic Sciences, 9 , no. 4 (1964): 413-27. Osterburg explored this issue and found large differences in examiners' perceived contribution for different minutia types and their configurations. However, Osterburg's study had significant limitations in its design and execution.

${ }^{163}$ Langenburg and Champod, 2011, op. cit.

${ }^{164}$ See, e.g., Tabassi, E. and C. Wilson. “A Novel Approach to Fingerprint Image Quality.” IEEE International Conference on Image Processing, 2 (September 2005): 37-40; Nill, N. "IQF (Image Quality of Fingerprint) Software Application.” MITRE (May 2007). Accessed April 22, 2011. http://www.mitre.org/tech/mtf/.
} 
for this software to be further tested and more readily available, however, some examiners have developed annotation schemes for indicating their personal assessments of confidence in the existence of a feature. For example, one scheme uses colors to represent an examiner's level of confidence. ${ }^{165}$ Similar to a traffic light, green features represent high confidence, yellow features represent medium confidence, red features represent low confidence, and orange features are those observed during the Comparison phase. This report does not offer specific prescriptions regarding which approaches or technologies should be used; however, tools that make explicit documentation both more routine and less time-consuming for examiners offer significant benefits to the interpretive process.

If time were no limitation, elaborate, formal documentation of all discriminative features in advance of a comparison would be ideal. The examiner would document all relevant and significant features observed and would record the confidence regarding these features before engaging in comparison. This annotation in advance would make explicit whether any significant features were noted only after the comparison had begun, whether the examiner's confidence in the existence of minutiae changed as a result of the comparison process, and whether these changes were justified. It would make the examiner's internal thought process more transparent to later observers and more transparent to the examiner himself.

However, not every comparison is complex, and time and resources are limited. Heavy caseloads are the norm for many forensic service providers. Therefore, the benefits of detailed and formalized documentation requirements must be balanced against the time and resources they require. Recommendation 3.1, regarding the importance of documentation to maintain transparency, which is highly relevant to feature selection, attempts to provide this balance by requiring some degree of contemporaneous documentation in all cases while also recognizing that the degree and detail of documentation may vary with the complexity of the print and the comparison.

\section{Variation}

Intra-examiner reliability refers to reproducibility on the part of the same examiner. Intraexaminer reliability would be present during feature selection when an examiner selects certain features in a questioned latent print at one time and selects different features from the same print at a later time (without recollection of the initial features selected). Inter-examiner reproducibility refers to the variation that exists across different examiners-variation that occurs when different examiners are presented with the same latent print and independently select different features. Studies of intra-examiner and inter-examiner variation reveal significant differences. ${ }^{166}$ Methods to reduce variation in feature selection are currently being explored and tested. ${ }^{167}$ In the meantime, because inter-examiner variation can be extensive, an examiner cannot assume that another examiner will see the same features (or place the same level of

\footnotetext{
${ }^{165}$ Langenburg and Champod, 2011, op.cit.

${ }^{166}$ Evett, I. and R. Williams. "A Review of the Sixteen Points Fingerprint Standard in England and Wales." Fingerprint Whorld, 82 (1995): 125-43; Langenburg, G. "Pilot Study: A Statistical Analysis of the ACE-V Methodology_Analysis Stage.” Journal of Forensic Identification, 54 (2004): 64-79; Dror, I., C. Champod, G. Langenburg, et al. "Cognitive Issues in Fingerprint Analysis: Inter- and Intra-Expert Consistency and the Effect of a 'Target' Comparison.” Forensic Science International, 208 (2011): 10-17.

${ }^{167}$ Langenburg, G., C. Champod, T. Genessay, et al. "Informing the Judgments of Fingerprint Analysts Using Quality Metric and Statistical Assessment Tools." National Institute of Justice Impression and Pattern Evidence Symposium, Clearwater, Florida. August 2010.
} 
confidence in the existence of any given feature). ${ }^{168}$ Documentation of the feature selection process is critical to understanding the differences among examiners and their decisions.

\section{Examination Complexity}

Not all print comparison tasks are equal in difficulty. Some comparisons are simple; others are far more complex. Although there is no formal method or metric for assessing complexity, experience and common sense point to some of the relevant factors. Examples of factors that lead to complexity include significant elements of distortion, reduced image quality (clarity), few reliable features available for comparison, or unusual, rare features to consider.

As described in Recommendation 3.1, the amount of documentation needed may vary depending on the complexity of the particular examination. In fairly simple, unambiguous cases, examiners appear to be fairly resistant to certain bias effects. ${ }^{169}$ Furthermore, although extensive interexaminer variation in feature selection appears to exist, some research suggests that this variation does not have significant effects on the ultimate decisions in noncomplex cases, but it is more significant in complex cases. ${ }^{170}$

These findings comport with intuition. When information is clear and plentiful, different examiners may elect to focus on different minutiae and yet reach consistent results. For the sake of efficiency, in these simple cases with significant quantity and quality of features present, minimal documentation may be sufficient because the risk of error due to bias or other human factors is also fairly minimal. Conversely, in complex cases, with limited quantity and quality of features, research shows that a variety of factors can influence interpretation and decisions. ${ }^{171}$ In these more difficult interpretations, documenting all available and reliable features and following stricter protocols regarding the analysis of the latent print before exposure to an exemplar can be critical in reducing the potential for error.

However, if documentation is permitted to vary with the complexity of the examination, examiners need to be able to determine complexity. It then becomes very important during the Analysis phase that the examiner both assesses and documents the complexity of the case. Current research is attempting to develop tools and metrics for quantitatively assessing the difficulty or complexity of a latent print comparison. In the meantime, an examiner's subjective assessment of case complexity can, by necessity, substitute, but this judgment must be documented in the case record. Some agencies may elect to institute a quality assurance policy or minimum minutiae threshold to assist in deciding how to document a case. For example, a

\footnotetext{
${ }^{168}$ An illustration can be found in the responses of the dozens of examiners in the public judicial inquiry set up by Scottish Ministers to verify the fingerprints associated with the case of H.M Advocate v. McKie in 1999, and related matters. Many chartings of the disputed latent print Y7 were submitted into evidence. Experts in the case disagreed about which features were reliable. Transcripts and court exhibits can be viewed at www.thefingerprintinquiryscotland.org.uk, accessed June 2, 2011.

${ }^{169}$ Dror, I., A. Peron, S. Hind, et al. "When Emotions Get the Better of Us: The Effect of Contextual Top-Down Processing on Matching Fingerprints.” Applied Cognitive Psychology, 19 (2005): 799-809; Dror, I. and D. Charlton. "Why Experts Make Errors." Journal of Forensic Identification, 56, no. 4 (2006): 600-16.

${ }^{170}$ Evett and Williams, op. cit.; Dror, Charlton, and Peron, op. cit.; Langenburg, G., C. Champod, and P. Wertheim. "Testing for Potential Contextual Bias Effects During the Verification Stage of the ACE-V Methodology When Conducting Fingerprint Comparisons.” Journal of Forensic Sciences, 54, no. 3 (May 2009): 571-82.

${ }^{171}$ Stacey, R. "A Report on the Erroneous Fingerprint Individualization in the Madrid Train Bombing Case." Journal of Forensic Identification, 54, no. 6 (2004): 706-18; Dror, Charlton, and Peron, op. cit.
} 
forensic service provider might require examiners to document all features during the Analysis phase when a latent print has fewer than 12 minutiae.

\section{Recursive Practice}

Once the examiner enters the Comparison phase and compares the latent print against an exemplar print (beginning with step 500 on the Latent Print Examination Process Map), looking at the exemplar may cause the examiner to re-evaluate aspects of the latent print. The examiner might use features in the exemplar print to find new features or to reassess previously noted features in the latent print. This practice, which has been referred to as "teasing the points"172 when used to attain a required minimum number of minutiae, poses possible concerns about bias. Some have criticized it as a form of circular reasoning because it uses the exemplar to infer meaning from the source print and vice versa. ${ }^{173}$ However, there are occasions when the exemplar may legitimately draw clearer attention to an important feature or reveal that some detail in the latent print previously thought to be "noise" is actually "signal."

Some sources, describing the advantages of a more cyclical process, defend examinations that move back and forth between latent and exemplar prints, ${ }^{174}$ whereas other sources discourage it in favor of a more linear approach. ${ }^{175}$ The difficulty is that the comparison print may lead both to accurate and inaccurate judgments about previously unnoticed features of the latent print, and there is no foolproof way to tell the two apart. Eliminating recursion would protect against picking or trimming the data to fit the conclusion, but it would also prevent the examiner from using valuable information. Moreover, recursive examinations may be especially important for reaching the conclusion to exclude. If the examiner sees several similarities between a latent and exemplar, notices another feature in the potential source print, returns to the latent, and does not see the corresponding feature, then the examiner is engaging in a form of recursion; yet even those who criticize recursive examinations would likely not wish to prevent such an analysis.

Recently, Dror et al. ${ }^{176}$ and Langenburg et al. ${ }^{177}$ proposed using features in the latent print that were selected after observing the exemplar only if the nonlinearity of the process is documented. These sources recommend that if these features are used, they must be formally documented as features observed during, rather than prior to, the Comparison phase, and typically they should be assigned less weight than if the feature had been observed initially in the Analysis phase. This documentation-focused compromise has significant merit. This approach is captured in Recommendation 3.2, which states that any modification to an analysis after examining the exemplar (both in feature selection and in other interpretive phases) should be formally documented.

\footnotetext{
${ }^{172}$ Evett and Williams, op. cit.; Tiller, C. "Fingerprint Identification in the United Kingdom-Do They Really Need 16 Points?" Canadian Identification Society Newsletter, 39 (1983): 11.

${ }^{173}$ IEEGFI II, op. cit.; U.S. Department of Justice, Office of the Inspector General, op. cit., p. 138.

${ }^{174}$ Vanderkolk, 2009, op. cit.; Triplett, M. and L. Cooney. "The Etiology of ACE-V and Its Proper Use: An Exploration of the Relationship Between ACE-V and the Scientific Method of Hypothesis Testing." Journal of Forensic Identification, 56, no. 3 (2006): 345-55.

${ }^{175}$ Haber, L. and R. Haber. Challenges to Fingerprints: A Guidebook for Prosecution and Defense and Examiners. Lawyers and Judges Publishing Co., 2009; IEEGFI II, op. cit.

${ }^{176}$ Dror, Champod, Langenburg, et al., op. cit.

${ }^{177}$ Langenburg and Champod, 2011, op . cit.
} 


\section{Cognitive Bias}

Various studies have shown that context information or other external factors can influence feature selection. ${ }^{178}$ However, some of this research has shown that not all bias effects are necessarily negative. At times, the biases can create more conservative decisions by examiners, even during feature selection. Whether greater conservatism is a plus or a minus depends on factors not yet fully understood (such as error rates in a variety of circumstances as well as policy decisions regarding tradeoffs between Type 1 and Type 2 errors). More study of the risks - and any benefits - of these bias effects and how to minimize them is warranted. One advisable countermeasure for bias in feature selection is simply to select features in the latent print separately from the exemplar. More generally, sequential unmasking, which excludes irrelevant contextual information, is a valuable strategy.

\subsubsection{Research Needs in Feature Selection}

\section{Assessing Variation in Feature Selection}

A handful of studies have assessed variation in the feature selection process. Generally, they find a wide variation among examiners during the task of feature selection. At this stage, what may be of more interest to the community are methods to reduce the variation of feature selection and tools and technologies to help identify the most reliable features. These tools are likely to be especially important for complex comparisons, and they may have benefits more broadly.

\section{Link between Feature Selection and Source Attribution}

The link between variations in feature selection and the examiner's ultimate decision is not well understood. Emerging research suggests that there is a relationship, ${ }^{179}$ but the effect may be competing with other major interpretative steps, such as interpreting discrepancy, feature weighting, and thresholds for reaching identification decisions. A better understanding of the link between feature selection and the ultimate decision would permit best practices to be formulated and would allow technology to select the most reliable and useful features on which to base decisions later in the ACE-V process.

What is clear from the research is that in complex cases, examiners who perceive and compare a greater number of features are more likely to reach a conclusion about source attribution (rather than determining the print to be inconclusive). Therefore, in complex cases, there is a strong need for tools or methods to standardize the selection of features. More generally, standardized methods for feature selection should result in less variation in the features that are selected and compared, which in turn should lead to more consistency in the decisions. However, even if decisions were more consistent, they would not necessarily be more accurate. It is possible, for example, that the best interpreters of prints would be more accurate using their own methods instead of following a standard protocol. This too, deserves attention and research.

\footnotetext{
${ }^{178}$ Evett and Williams, op. cit.; Dror, Charlton, and Peron, op. cit.; Langenburg, Champod, and Wertheim, op. cit.; Dror, Champod, Langenburg, et al., op. cit.

${ }^{179}$ Langenburg, Champod, Genessay, et al., op. cit.
} 


\subsection{Utility Assessment}

Several points in the latent print analysis process require a determination of latent print utility or sufficiency. The examiner must determine whether the print has enough clarity and quantity of information for some specified purpose. This happens both early in the process (e.g., determinations of whether the latent is suitable for analysis or suitable for an AFIS search) and later in the process (e.g., determinations of sufficient information to compare the latent and exemplar). For convenience, the common points are discussed here, since the recurring question is whether there is sufficient information for making the next inquiry.

\subsubsection{Corresponding Steps in the Latent Print Examination Process Map}

Key points at which utility determinations are relevant are discussed here with the numbers in parentheses referring to steps in the Latent Print Examination Process Map. Upon acquiring a latent print, an examiner must determine whether it is suitable for comparison (step 240). This typically involves examining the details and any relevant distortion in the latent print to determine whether there is enough usable information to make a comparison viable. Assuming the latent print is suitable and that known prints are available, the examiner next determines if the known prints are suitable for comparison (step 350) and if the two prints combined are sufficient to see the comparison through to a decision (step 370). If no known prints are available, the examiner may be called on to determine if the latent print is suitable for an AFIS search (step 270).

\subsubsection{Findings from Existing Research}

The Working Group found no research that effectively addresses utility or sufficiency in the context of fingerprint analysis. This is unsurprising, for a critical piece for any such researchthe definition and validation of a metric for assessing utility - is still missing. Research has been carried out related to the examiner decision process that implicitly involves judgments of utility and sufficiency, but these studies tend to focus only on the accuracy or repeatability of the ultimate decision and thus are reviewed in the relevant sections below.

\subsubsection{Recommendations and Best Practices}

The absence of research findings specific to the determination of utility precludes evidence-based recommendations to guide practitioners. Instead, the primary findings with regard to utility determination concern practices to encourage consistent decision-making and to allow for ongoing evaluation of quality both within and across forensic service providers. This involves Recommendation 3.1, regarding the importance of documentation, and Recommendation 3.2, regarding the special importance of documenting any utility determinations that are altered after examining the exemplar. Such documentation is critical in utility determinations because it allows for internal quality control and quality assurance. It also is of great importance for any potential future external evaluations of quality.

Agencies and forensic service providers can enhance the transparency of their analyses and conclusions by determining clear guidelines that assist examiners in making determinations of utility. This refers to guidelines for deciding whether a print warrants further assessment and not

53 Latent Print Examination and Human Factors: Improving the Practice through a Systems Approach

The Report of the Expert Working Group on Human Factors in Latent Print Analysis 
to guidelines for making ultimate source attribution. For example, an agency might require at least five minutiae to conduct an AFIS search.

This chapter does not propose particular guidelines for determining utility but suggests that each forensic service provider try to make this step as explicit as possible. Until a substantial research basis exists from which best practices can be generated, it is inappropriate to require field-wide shared standards. Nevertheless, forensic service providers can draw on experience to establish clear guidelines. Information that could be incorporated in an agency's guidelines includes number of minutiae and visibility of ridge flow, pores, ridge edges, and details of incipient ridges, creases, and scars. The guidelines for determining whether a latent print is suitable for comparison are necessarily different from the guidelines for comparing a pair of prints.

\section{Recommendation 3.4: Each agency or forensic service provider should define "suitable" or "sufficient" in its standard operations procedures. These guidelines should be as explicit as possible about what is expected for sufficiency determinations at different stages of the latent print examination process.}

The Working Group takes no position on whether agencies should couch guidelines as recommendations or as requirements.

\subsubsection{Research Needs in Utility Assessment}

Existing research has not resolved the questions of utility, sufficiency, and value determination in latent print analysis. The Working Group strongly endorses further research in this area to enable the development of evidence-based guidelines and recommendations for utility determination. Although a general discussion of the need for research and a recommendation regarding research is provided near the end of the chapter, some preliminary thoughts regarding research questions relevant to utility or value determinations are provided here.

Important areas for study include the following:

- Reproducibility and accuracy: It is of great interest to understand the degree to which utility determinations are reliable. For each utility determination, researchers could present the same print at different times to a single examiner, present the same print to different examiners, or both. These studies could provide significant information about consistency, though they would not tell the researcher anything directly about accuracy. Assessing the accuracy of utility determinations is more problematic because there is no objective basis for ascertaining when a determination is correct.

- Factors affecting utility determinations: A wide range of factors can affect utility determinations. One possible topic for consideration is context effects: does the type of case (e.g., violent crime versus property crime) have an effect on the determination of utility, or does background knowledge about the case affect utility judgments? Another possible topic is comparator effects: are different utility determinations obtained for comparing latent prints with suspect prints as opposed to comparing latent prints with AFIS-generated targets? Other factors that should be studied include forensic service provider culture norms and other human factors. 
- The effect of laboratory culture: The relationship between the culture and expectations in an agency and examiner performance has not been studied. For example, do laboratories that frown on inconclusive determinations eliminate more prints at an earlier utility stage?

- Utility effects with relation to manual, as opposed to AFIS, search strategies: Research could investigate, for example, whether certain pattern types create different utility strategies in relation to searching manually or on AFIS.

\subsection{Feature Weighting}

Practitioners recognize that assigning weights to latent print features and their configuration is a fundamental decision-making or interpretive step, either as an output of the Analysis phase or as part of the Evaluation phase. Pioneers such as Locard indicated that fingerprint features should be assessed as a function of their relative rarity (and clarity). ${ }^{180}$

\subsubsection{Frequencies of Friction Ridge Details}

As discussed in section 3.1, the weighting of individual features currently reflects subjective, experience-based judgments of the probability associated with their occurrence. Whether done subjectively or in a more data-oriented fashion, weighting is a probabilistic assignment of the selectivity of the features being considered. The process of weighting among practitioners today, however, remains essentially holistic, and the subjective probabilities are rarely made explicit. ${ }^{181}$ In addition, no clear consensus exists among practitioners as to the reproducibility and perceived contribution of L3D features appearing in both latent and exemplar prints. ${ }^{182}$

Although individual experience may provide some insight into probabilities, carefully collected data should be used to provide more accurate assessments that can inform the weighting of features in the interpretive process. To demonstrate the nature and value of such data, this section summarizes the major sources of data available on the classification and prevalence of L1D features (general flow of the ridges) and L2D features (here restricted to minutiae) for fingerprints. ${ }^{183}$ Limited statistical data are available for L3D features of fingerprints. Other types of friction ridge impressions (palms, toes, etc.) do not yet have comparable data available for even L1D features.

\section{L1D Features}

The prevalence of different types of L1D features in the population is well documented. Dermatoglyphic data from studies in anthropology, population genetics, and early detection of

\footnotetext{
${ }^{180}$ Locard, E. "La Preuve Judiciaire par les Empreintes Digitales.” Archives d'Anthropologie Criminelle, de Médecine Légale et de Psychologie Normale et Pathologique, 29 (1914): 321-48.

${ }^{181}$ In fact, in one early study, latent print examiners did not provide consistent rankings of how frequently fingerprint features occur. Osterburg, op. cit.

${ }^{182}$ Anthonioz, A., N. Egli, C. Champod, et al. "Level 3 Details and Their Role in Fingerprint Identification: A Survey Among Practitioners." Journal of Forensic Identification, 58, no. 5 (2008): 562-89.

${ }^{183}$ Part of this section is based upon a report of C. Champod, provided on September 28, 2009, to the Scottish Ministers' Fingerprint Inquiry, available at www.thefingerprintinquiryscotland.org.uk/inquiry/2090.html, accessed April 22, 2011.
} 
disease, are one source. ${ }^{184}$ These data can be used in forensic science, but the classifications often do not match those used by forensic experts. Other data come from collections of ten-print cards. The majority of large collections were classified according to the Galton-Henry scheme, ${ }^{185}$ as illustrated in Figure 3.1:

\begin{tabular}{|c|c|c|c|}
\hline Arches & Tented Arches & Right Loops & Left Loops \\
\hline 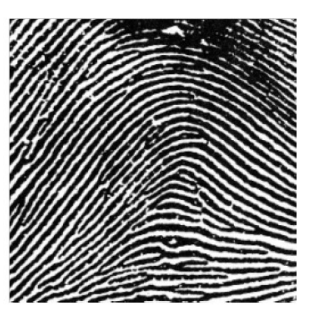 & Jysuly & 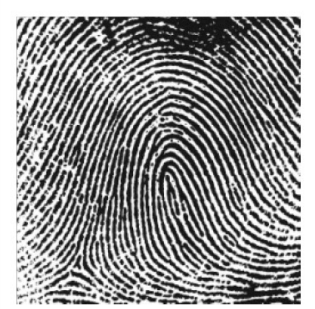 & 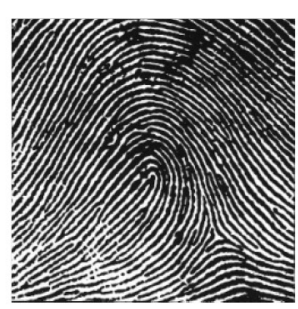 \\
\hline
\end{tabular}

\begin{tabular}{|c|c|c|c|}
\hline Plain Whorls & $\begin{array}{c}\text { Central Pocket } \\
\text { Loops }\end{array}$ & Double Loops & Accidental Whorls \\
\hline & & & \\
& & &
\end{tabular}

Figure 3.1: Level 1 Detail features ${ }^{186}$

\footnotetext{
${ }^{184}$ See, e.g., Kumbnani, H. “Dermatoglyphics: A Review.” In Anthropology Today: Trends, Scope and Applications, edited by M. Bhasin and V. Bhasin, 285-95. Kamla-Raj Enterprises, 2007.

${ }^{185}$ Henry, E. Classification and Uses of Finger Prints. 4th ed. George Routledge and Sons, 1900.

${ }^{186}$ Images adapted from Royal Canadian Mounted Police. Fingerprint Manual. Chapter 2. Royal Canadian Mounted Police, 1990.
} 
Data from the Federal Bureau of Investigation's National Crime Information Center permits estimates of the prevalence of each class of L1D in the U.S. population.

\begin{tabular}{|l|l|l|l|l|}
\cline { 2 - 5 } \multicolumn{1}{c|}{} & \multicolumn{2}{c|}{ Male Left Hand Patterns } & \multicolumn{2}{c|}{ Male Right Hand Patterns } \\
\hline Arches & $3.6 \%$ & & $2.9 \%$ & \\
\hline Tented Arches & $3.1 \%$ & & $2.7 \%$ & \\
\hline Right Loops & $3.9 \%$ & & $58.0 \%$ & \\
\hline Left Loops & $63.7 \%$ & & $4.4 \%$ & \\
\hline Pla in Whorls & $17.3 \%$ & & $24.4 \%$ & \\
\hline Central Pocket Loops & $3.8 \%$ & & $4.1 \%$ & \\
\hline Double Loops & $4.1 \%$ & & $3.2 \%$ & \\
\hline Accidental Whorls & $0.1 \%$ & & $0.083 \%$ & \\
\hline Missing or Amputated & $0.2 \%$ & & $0.2 \%$ & \\
\hline Scarred or Mutilated & $0.09 \%$ & & $0.094 \%$ & \\
\hline
\end{tabular}

Table 3.1: Distribution for the general patterns on fingerprints from the left and right hands of males $(89,755,960 \text { fingers })^{187}$

The relative frequencies of L1D can vary depending on the finger considered and, to some degree, the sex and ethnic background of the population. For example, comparing right thumbs to the little fingers of males produces the distributions in Table 3.2.

\begin{tabular}{|l|l|l|l|l|}
\cline { 2 - 5 } \multicolumn{1}{c|}{} & \multicolumn{2}{c|}{ Male Right Thumb Patterns } & \multicolumn{2}{c|}{ Male Right Little Finger Patterns } \\
\hline Arches & $2.5 \%$ & & $0.7 \%$ & $\mid$ \\
\hline Tented Arches & $0.4 \%$ & & $0.6 \%$ & $\mid$ \\
\hline Right Loops & $50.8 \%$ & & $82.3 \%$ & \\
\hline Left Loops & $0.4 \%$ & & $0.3 \%$ & \\
\hline Pla in Whorls & $34.8 \%$ & & $11.8 \%$ & \\
\hline Central Pocket Loops & $1.1 \%$ & & $3.6 \%$ & \\
\hline Double Loops & $9.9 \%$ & & $0.4 \%$ & $\mid$ \\
\hline Accidental Whorls & $0.021 \%$ & & $0.009 \%$ & \\
\hline Missing or Amputated & $0.088 \%$ & & $0.1 \%$ & \\
\hline Scarred or Mutilated & $0.025 \%$ & & $0.059 \%$ & \\
\hline
\end{tabular}

Table 3.2: Distribution for the general patterns on fingerprints from the right thumb and little fingers of males $(17,951,192 \text { fingers })^{188}$

Some classes of L1D can be subdivided according to ridge counts (number of ridges between the core of the pattern to the delta) and ridge tracing (relative positioning of the deltas). Statistics in relation to these subclassifications are available. Table 3.3 presents two examples.

${ }^{187}$ Champod, C., C. Lennard, P. Margot, et al. Fingerprints and Other Ridge Skin Impressions. CRC Press, 2004.

${ }^{188} \mathrm{Ibid}$. 


\begin{tabular}{|c|c|c|c|c|c|}
\hline \multicolumn{3}{|c|}{$\begin{array}{c}\text { Distribution for the subclasses of ridge counts } \\
(0-10 ; 11-20 ; 21-30 ; 31-40 ; 40+) \text { for right loops of } \\
\text { males }(55,541,294 \text { fingers }) \text {. }\end{array}$} & \multicolumn{3}{|c|}{$\begin{array}{l}\text { Distribution for the subclasses of ridge tracing } \\
\text { (Inside, Meet, Outside) for plain whorls of males } \\
(37,421,395 \text { fingers). }\end{array}$} \\
\hline $0-10$ & $37.0 \%$ & (2) & \multirow{2}{*}{ Inside } & \multirow{2}{*}{$40.2 \%$} & \\
\hline $11-20$ & $56.7 \%$ & & & & \\
\hline $21-30$ & $6.1 \%$ & 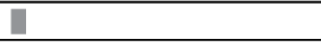 & \multirow{2}{*}{ Meet } & \multirow{2}{*}{$19.9 \%$} & \\
\hline $31-40$ & $0.027 \%$ & & & & \\
\hline $40+$ & $0.00095 \%$ & & Outside & $39.9 \%$ & \\
\hline
\end{tabular}

Table 3.3: Examples of statistics on subclassifications ${ }^{189}$

These data can assist fingerprint examiners in several ways. Because the frequencies of general patterns vary across the different fingers, the patterns offer information on which finger the latent print came from. In addition, during the Evaluation phase, a rare general pattern, such as a tented arch, can be weighted more than a common general pattern, such as a loop with a ridge count of between 11 and 20 .

\section{L2D Features}

The following two tables list frequencies, reported in studies prior to 1985, of the more discriminating L2D features (especially minutiae). ${ }^{190}$ Not all types of minutiae were considered in each study. ${ }^{191}$

\begin{tabular}{|c|c|c|c|c|}
\hline \multirow[b]{2}{*}{ Minutia Type } & \multirow[b]{2}{*}{ Example } & \multicolumn{3}{|c|}{ Study Authors, (Number of Prints) Number of Minutiae } \\
\hline & & $\begin{array}{c}\text { Gupta, }(1,000 \\
\text { ulnar loops) } \\
\text { Unknown number } \\
\text { of minutiae }\end{array}$ & $\begin{array}{c}\text { Osterburg et al., } \\
\text { (39 fingerprints) } \\
8,591 \text { cells of } 1 \\
\text { mm2 }\end{array}$ & $\begin{array}{l}\text { Lin et al., } \\
\text { (76 fingerprints) } \\
\text { 14,280 minutiae }\end{array}$ \\
\hline Ridge ending & & $7.50 \%$ & $8.32 \%$ & $9.60 \%$ \\
\hline $\begin{array}{l}\text { Bifurcation } \\
\text { opening }\end{array}$ & & $8.00 \%$ & $3.82 \%$ & $2.60 \%$ \\
\hline Deviation & & $0.90 \%$ & Not considered & Not considered \\
\hline Bridge & & $0.80 \%$ & $1.22 \%$ & Not considered \\
\hline Island & & $2.50 \%$ & $1.77 \%$ & $0.99 \%$ \\
\hline
\end{tabular}

\footnotetext{
${ }^{189}$ Ibid.

${ }^{190}$ Stoney, D. "Measurement of Fingerprint Individuality." In Advances in Fingerprint Technology, edited by Henry C. Lee and R.E. Gaensslen, 327-87. 2nd ed. CRC Press, 2001, p. 327.

${ }^{191}$ A more recent analysis appears in Gutièrrez, E., V. Galera, J. Martínez, et al. "Biological Variability of the Minutiae in the Fingerprints of a Sample of the Spanish Population.” Forensic Science International, 172 (2007): 98.
} 


\begin{tabular}{|c|c|c|c|c|}
\hline Minutia Type & Example & Gupta & Osterburg et al. & Lin et al. \\
\hline Interruption & & $0.90 \%$ & $1.39 \%$ & $1.20 \%$ \\
\hline Hook & & $2.00 \%$ & $0.75 \%$ & $0.48 \%$ \\
\hline Lake & & $2.50 \%$ & $0.64 \%$ & $0.17 \%$ \\
\hline Dot & & $3.50 \%$ & $1.51 \%$ & $0.52 \%$ \\
\hline Crossing & & $0.50 \%$ & Not considered & Not considered \\
\hline Transversal & & $0.50 \%$ & Not considered & Not considered \\
\hline Return & & $0.80 \%$ & Not considered & Not considered \\
\hline $\begin{array}{l}\text { Double } \\
\text { bifurcation }\end{array}$ & & Not considered & $0.14 \%$ & $0.27 \%$ \\
\hline Trifurcation & & Not considered & $0.09 \%$ & Not considered \\
\hline Angular line & & Not considered & Not considered & $0.17 \%$ \\
\hline Delta & & Not considered & $0.20 \%$ & Not considered \\
\hline $\begin{array}{l}\text { Other multiple } \\
\text { occurrence }\end{array}$ & & Not considered & $3.55 \%$ & Not considered \\
\hline $\begin{array}{l}\text { Absence of } \\
\text { minutiae }\end{array}$ & & Not considered & $76.60 \%$ & $83.90 \%$ \\
\hline
\end{tabular}

Table 3.4: Relative frequencies for different types of minutiae according to Gupta (1968), ${ }^{192}$ Osterburg et al. (1977), ${ }^{193}$ and Lin et al. (1982) ${ }^{194,} 195$

${ }^{192}$ Gupta, S. "Statistical Survey of Ridge Characteristics." International Criminal Police Review, 218 (1968): 130. 


\begin{tabular}{|c|c|c|c|c|}
\hline \multirow[b]{2}{*}{ Minutia Type } & \multicolumn{4}{|c|}{ Study Authors, (Number of Prints) Number of Minutiae } \\
\hline & $\begin{array}{c}\text { Santamaria } \\
\text { Beltran, }(1,000 \\
\text { fingerprints }) \\
\text { Unknown } \\
\text { number of } \\
\text { minutiae }\end{array}$ & $\begin{array}{c}\text { Kingston, (100 } \\
\text { ulnar loops) } \\
2,464 \text { minutiae }\end{array}$ & $\begin{array}{c}\text { Sclove, (39 } \\
\text { fingerprints) } \\
2,536 \text { minutiae }\end{array}$ & $\begin{array}{c}\text { Stoney, }(412 \\
\text { thumbs-dista } \\
\text { area) } 2,645 \\
\text { minutiae }\end{array}$ \\
\hline Ridge ending & $53.40 \%$ & $45.90 \%$ & $49.70 \%$ & $56.80 \%$ \\
\hline $\begin{array}{l}\text { Bifurcation } \\
\text { opening }\end{array}$ & $15.10 \%$ & $34.10 \%$ & $15.90 \%$ & $43.20 \%$ \\
\hline $\begin{array}{l}\text { Bifurcation } \\
\text { closing }\end{array}$ & $13.10 \%$ & Not considered & Not considered & Not considered \\
\hline Deviation & $2.20 \%$ & Not considered & Not considered & Not considered \\
\hline Bridge & $1.30 \%$ & $1.90 \%$ & $5.58 \%$ & Not considered \\
\hline Island & $5.40 \%$ & Not considered & $10.30 \%$ & Not considered \\
\hline Interruption & $1.60 \%$ & Not considered & Not considered & Not considered \\
\hline Hook & Not considered & Not considered & $3.50 \%$ & Not considered \\
\hline Lake & $4.20 \%$ & $3.20 \%$ & $2.63 \%$ & Not considered \\
\hline Dot & $2.20 \%$ & $8.30 \%$ & $10.20 \%$ & $5.30 \%$ \\
\hline Crossing & $0.22 \%$ & Not considered & Not considered & Not considered \\
\hline Transversal & $1.30 \%$ & Not considered & Not considered & Not considered \\
\hline Return & $0.024 \%$ & Not considered & Not considered & Not considered \\
\hline Double bifurcation & Not considered & Not considered & $0.64 \%$ & Not considered \\
\hline Trifurcation & Not considered & Not considered & $0.28 \%$ & Not considered \\
\hline Delta & Not considered & $1.70 \%$ & $1.35 \%$ & Not considered \\
\hline $\begin{array}{l}\text { Other multiple } \\
\text { occurrence }\end{array}$ & Not considered & $3.10 \%$ & Not considered & Not considered \\
\hline
\end{tabular}

Table 3.5: Relative frequencies for different types of minutiae according to Santamaria Beltran (1953), ${ }^{196}$ Kingston (1964), ${ }^{197}$ Sclove (1979-1980), ${ }^{198}$ and Stoney $(1985){ }^{199}$

\footnotetext{
${ }^{193}$ Osterburg, J., T. Parthasarathy, T. Raghavan, et al. "Development of a Mathematical Formula for the Calculation of Fingerprint Probabilities Based on Individual Characteristics." Journal of the American Statistical Association, 72 (1977): 772.

${ }^{194}$ Lin, C., J. Liu, J. Osterburg, et al. "Fingerprint Comparison I: Similarity of Fingerprints." Journal of Forensic Science, 27, no. 2 (1982): 290.

${ }^{195}$ Images adapted from Champod, C. Reconnaissance Automatique et Analyse Statistique des Minuties sur les Empreintes Digitales. PhD Thesis. Institut de Police Scientifique et de Criminologie, Université de Lausanne, Lausanne, Suisse, 1996.

${ }^{196}$ Santamaria Beltran, F. "Une Nouvelle Méthode d'Évaluation des Points Caracteristiques des Crêtes Papillaires." Assemblée Générale de la Commission International de Police Criminelle - Oslo. $24-29$ June 1953; Santamaria Beltran, F. "Sous-Commission á la Dactyloscopie, Nouveaux Concepts d'Évaluation des Points Caractéristiques de F. Santamaria Beltran." Assemblée Générale de la Commission International de Police Criminelle - Oslo. 25 June
} 
Although this information could be helpful to an examiner, the data have significant limitations. There is no standardized nomenclature for the type of minutiae considered, how they were counted, how the absence of minutiae was evaluated, or how minutiae were distinguished from one another (e.g., when two bifurcations should be counted as two events or one double bifurcation event). This lack of standardized terminology applies across the entire fingerprint comparison field ${ }^{200}$ and makes comparison across these studies extremely challenging.

Also, the density of minutiae and their relative proportions depends greatly on their positioning on the papillary surface (especially in relation to core and deltas). For example, areas such as deltas and ridge flow regions along "type lines" tend to produce a higher number of minutiae. None of the studies took this into account. More recent models have begun to account for this phenomenon by using data-driven approaches that do not rely on pre-defined categories. ${ }^{201}$

\section{L3D Features ${ }^{202}$}

Limited statistical analyses of pores, or by extension, of L3D features of fingerprints, have been carried out for latent print comparisons in a forensic context. Ashbaugh indicated that 20 to 30 corresponding pores between a latent print and a reference print would be sufficient for an individualization. ${ }^{203}$ Roddy and Stosz concurred. ${ }^{204}$ However, their models relied on questionable independence assumptions ${ }^{205}$ and, more importantly, did not take into account the potential lack of reproducibility of these features from one impression to the next. Parsons et al. investigated within-source variability and concluded that "due to contamination and inherent dependence, matching on 20 pores as has been previously suggested (Ashbaugh, 1983) appears to us overly optimistic. On the basis of this study, we would conjecture that good fingerprints with more

1953; Santamaria Beltran, F. “A New Method for Evaluating Ridge Characteristics.” Fingerprint and Identification Magazine, 36, no.11 (1955).

${ }^{197}$ Kingston, C. "Probabilistic Analysis of Partial Fingerprint Patterns." Doctorate of Criminology diss., University of California, Berkeley, 1964.

${ }^{198}$ Sclove, S. "The Occurrence of Fingerprint Characteristics as a Two-Dimensional Process." Journal of the American Statistical Association, 74 (1979): 588; Sclove, S. "The Occurrence of Fingerprint Characteristics as a Two-Dimensional Poisson Process." Communications in Statistics - Theoretical Methods, A9 (1980): 675.

${ }^{199}$ Stoney, D. “A Quantitative Assessment of Fingerprint Individuality.” PhD thesis, University of California, Davis, 1985 .

${ }^{200}$ Saviers, K. "Friction Skin Characteristics: A Study and Comparison of Proposed Standards." Journal of Forensic Identification, 39 (1989): 157.

${ }^{201}$ Champod, C. and P. Margot. "Computer Assisted Analysis of Minutiae Occurrences on Fingerprints." Proceedings of the International Symposium on Fingerprint Detection and Identification. 1995; Neumann, Champod, Puch-Solis, et al., op. cit.; Egli, N. "Interpretation of Partial Fingermarks Using an Automated Fingerprint Identification System.” PhD thesis, École des Sciences Criminelles, University of Lausanne, 2009; Su, C. and S.

Srihari. "Probability of Random Correspondence for Fingerprints." Computation Forensics: Lecture Notes in Computer Science, 5718 (2009): 55.

${ }^{202}$ This section comes from the supplementary report C. Champod provided on October 21, 2009, to The Fingerprint Inquiry of Scotland, available at www.thefingerprintinquiryscotland.org.uk/inquiry/2090.html, accessed April 22, 2011.

${ }^{203}$ Ashbaugh, D. "Poroscopy." RCMP Gazette, 45, no. 2 (1983): 12.

${ }^{204}$ Roddy and Stosz, op. cit.

${ }^{205}$ Parsons, N., J. Smith, E. Thönnes, et al. "Rotationally Invariant Statistics for Examining the Evidence from the Pores in Fingerprints." Law Probability \& Risk, 7, no. 1 (2007): 1.

61 Latent Print Examination and Human Factors: Improving the Practice through a Systems Approach The Report of the Expert Working Group on Human Factors in Latent Print Analysis 
than about 80 pores appear to have potentially strong evidential value using this extraction algorithm." 206

Some biometric identification systems use pores and other features, such as dots and incipient ridges, to increase the efficiency of matching algorithms based on minutiae. ${ }^{207}$ However, highquality input prints increase the performance of such systems; L3D may have minimal added value for forensic latent prints of varying clarity and degrees of distortion.

\subsubsection{Recommendations and Best Practices}

Data on minutiae rarity, density, and location should be important to latent print examiners in weighting different types of minutiae. These data can contribute to an expert's informal judgment on the relative frequency of a given minutia. At the moment, surveys providing such information are available only for fingerprints, not for other forms of friction ridge areas such as palms or feet. Furthermore, the above data illustrate that a simple additive rule with equal weight for each minutiae within a fingerprint, such as a 12-point standard, ignores statistically important information. The data clearly show, as many examiners well recognize, that all minutiae are not equally useful for interpretation. For example, it may be seven times more common to see a ridge ending within a delta zone than a hook. The amount of discriminating information provided by the former is thus significantly less than that provided by the latter. Future research could better quantify the weight that should be assigned to different types of minutiae. Educating examiners about existing statistics, the limitations of these data, and the potential value of better statistics will advance the field.

\section{Recommendation 3.5: Because statistical information plays a fundamental role in weighting latent print feature evidence, training should include the best available empirical information and should educate examiners about probabilistic reasoning in using that information.}

Given the current limitations in data and probabilistic models, other difficulties in weighting fingerprint evidence arise in some circumstances. Modern investigations often compare latent prints to exemplars from AFIS searches. Such exemplars raise the possibility of increased similarity to a latent print that actually comes from a different source. This phenomenon has been called "incidental similarity." 208 Of course, the computer programs that generate candidates compare prints differently than human examiners do; nevertheless, when looking in a large database for those prints that bear the greatest similarity to the latent print in question, these

\footnotetext{
206 Ibid.

${ }^{207}$ Chen, Y. and A. Jain. "Dots and Incipients: Extended Features for Partial Fingerprint Matching.” Paper presented at the Biometrics Symposium 2007; Kryszczuk, K., A. Drygajlo, and P. Morier. "Study of the Distinctiveness of Level 2 and Level 3 Features in Fragmentary Fingerprint Comparison." Presented at the Proceedings of Biometric Authentication Workshop, ECCV, Prague, Czech Republic 2004, 124-33; Jain, Chen, and Demirkus, op. cit.; Zhao, Q., D. Zhang, L. Zhang, et al. "High Resolution Partial Fingerprint Alignment Using Pore-Valley Descriptors." Pattern Recognition, 2009; Vatsa, M., R. Singh, A. Noore, et al. "Combining Pores and Ridges with Minutiae for Improved Fingerprint Verification.” Signal Processing, 89, no. 12 (2009): 2676-85.

${ }^{208}$ For a discussion of this issue see Dror, I. and J. Mnookin. "The Use of Technology in Human Expert Domains: Challenges and Risks Arising From the Use of Automated Fingerprint Identification Systems in Forensic Science." Law, Probability \& Risk, 9 (2010): 47-67.
} 
programs could select a print from a nonsource that is far more similar to the latent print than examiners are accustomed to seeing. ${ }^{209}$

An examiner whose idea of the necessary amount of information for a match comes from a nondatabase world may not be taking into account the possibility of incidental similarity that a large database carries with it. Unfortunately, no one has studied whether incidental similarity in a database context is a substantial problem, ${ }^{210}$ and it is tricky to recommend modifications to an interpretive system that is largely non-formalized. However, the Working Group believes that interpreting the quality of the evidence in such cases requires special care because the chances of finding a close nonmatch are higher. Such care might include strategies such as being more explicit about the weight assigned to individual features, using a higher threshold before reaching the conclusion for a match, or narrowing the tolerances for visual differences in appearance. These considerations lead to the following recommendation:

\section{Recommendation 3.6: When comparing latent prints to exemplars generated through AFIS searches, examiners must recognize the possibility and dangers of incidental similarity. Adjustments such as a higher decision threshold, stricter tolerances for differences in appearance, and explicit feature weighting need to be considered. Modifying quality assurance practices for this scenario also should be considered.}

\subsubsection{Research Needs in Feature Weighting}

There is a strong need for systematic studies pertaining to the reproducibility and discriminating strength of fingerprint features. More specifically, although the prevalence of particular L1D features is well documented, a more complete understanding of the dependencies in these features between fingers is desirable. Regarding L2D features, there is limited research that would allow a global assessment (without strong independence assumptions) of the strength of minutiae configurations. Likewise, the information power of L3D features is not fully understood nor has it fully been explored for forensic use. Limited systematic studies have been carried out on features such as creases, lines, and scars, which are useful to support the evaluation process when these features are present. These studies should take into account variables such as sex, finger number, pattern, and race.

Such research is important when the examiner subjectively assigns weight to a set of features. Empirical studies on rarity can inform these experience-based judgments and help allow an examiner to assign appropriate weights to sets of features. Moreover, research exploring how examiners overestimate or underestimate the significance of features as a function of training, motivation, or context of the examination would be useful.

\footnotetext{
209 Ibid.

${ }^{210}$ But see Ulery, B., A. Hicklin, J. Buscaglia, et al. "Accuracy and Reliability of Forensic Latent Fingerprint Decisions." Proceedings of the NAS. 2011. This experiment found relatively few false identifications for a sample of latent prints drawn in part from the results of AFIS searches, discussed supra Chapter 2).
} 


\subsection{Discrepancy Interpretation}

In the friction ridge discipline, tolerance has been described as "the limits of how much distortion or difference is still considered within the parameters of agreement." 211 No two images, even if from the same source, will look identical. The question is the extent to which differences in the appearance of a particular friction ridge feature in the impression should be considered consistent with it coming from the same source as the exemplar print.

\subsubsection{Corresponding Stages in the Latent Print Examination Process Map}

The decision regarding distortion occurs in several places in the latent print examination process. During the Analysis phase, an examiner observes not only the distinctive details of the latent print (step 210), but also analyzes the relevant distortion present in the latent print (step 220). Factors that affect the appearance of a latent print include the pliability of skin, condition of skin, deposition pressure, lateral movement, matrix (substance coating the ridges), substrate (surface on which the print is deposited), and development method. ${ }^{212}$ An important part of the initial analysis is the determination of how these factors might affect the latent print.

A similar, but separate, analysis is conducted on the known print (step 330). Pliability and condition of skin, recording method (e.g., ink or livescan), deposition pressure, lateral movement, and other factors can distort these impressions as well.

In the Comparison phase, an examiner observes the latent and known prints side by side. The examiner must decide whether to tolerate the observed dissimilarities at all three levels of detail. If the two prints cannot be excluded based on L1D (step 500), the examiner continues the comparison to determine if the previously selected target group (step 290) falls within tolerance in the known print (step 510). If so, the examiner continues comparing additional detail. As dissimilarities are detected during the process, the examiner must determine if those differences are within tolerance for identification (step 600).

\subsubsection{Findings from Existing Research}

To assess distortion accurately, examiners must understand the flexibility of the skin, the limits of skin deformation, and other factors that may affect the appearance of a friction ridge impression. Figure 3.2 illustrates the range of distortion that can be expected on prints left by the same finger.

\footnotetext{
${ }^{211}$ Ashbaugh, 1999, op. cit.

${ }^{212}$ Ibid.
} 


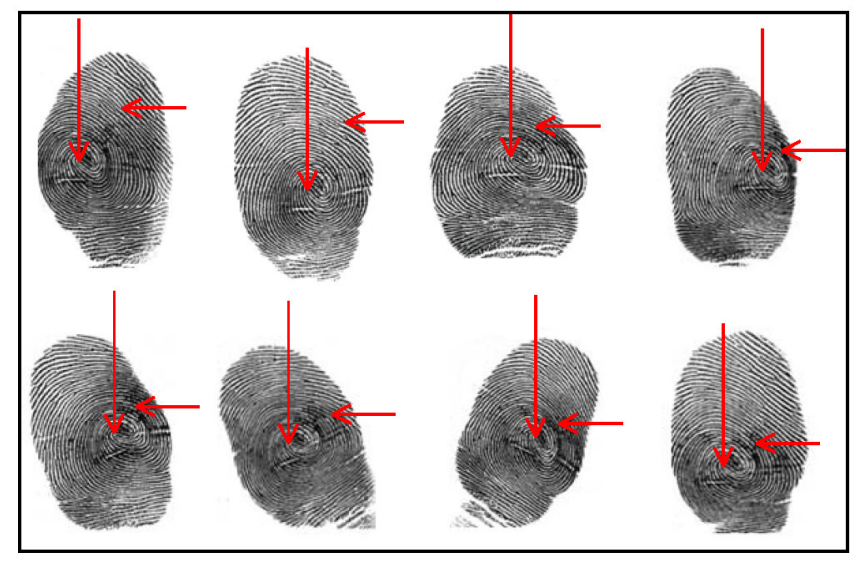

Figure 3.2: Examples of skin distortion on prints of the same finger with arrows indicating location of the same minutiae in different impressions ${ }^{213}$

In developing automated fingerprint recognition systems, the biometrics industry conducted considerable research into the flexibility of skin. ${ }^{214}$ However, nonlinear distortion is complex. Although some research examines the effect of different pressures and motions on friction ridge impressions, ${ }^{215}$ examiners must predominantly rely on the knowledge gained during training and subsequent experience to recognize and interpret distortion.

Under the one-dissimilarity doctrine, an examiner who does not know the cause of a dissimilarity must exclude the two prints as having come from the same source. ${ }^{216}$ Under this rule, an incorrect interpretation of just one dissimilarity could lead to an erroneous exclusion. Some examiners do not accept the "one-dissimilarity" doctrine, believing it to be too strict given thecomplexity of impressions and the difficulty of distinguishing "true" dissimilarities from other distortions. Even among those examiners who do accept the rule in theory, it is not clear how rigorously it operates in practice. Faced with overwhelming detail in agreement and only one dissimilarity, examiners may have an understandable tendency to rationalize away the dissimilarity, even if they are not confident about its cause. Research does not establish whether such rationalization increases the accuracy of ultimate decisions or decreases it. It is certainly possible that a willingness to explain away difference in the face of a large amount of similarity would lead, on balance, to greater accuracy. Confirmation bias - the tendency to give more weight to confirming rather than disconfirming information-has been observed in many human endeavors (see Chapter 2). This type of bias also could occur if information from the known print influences the interpretation of the latent print. To minimize this effect, a thorough analysis of the latent print should be conducted before analyzing the known print.

Although some literature demonstrates the effects that different distortions may have on friction ridge impressions, ${ }^{217}$ little research has tested the examiner's ability to accurately identify the

\footnotetext{
${ }^{213}$ Fingerprint images adapted courtesy of the Instiut de Police Scientifique, Université de Lausanne.

${ }^{214}$ Dorai, C., N. Ratha, and R. Bolle. "Dynamic Behavior Analysis in Compressed Fingerprint Videos." IEEE Transactions on Circuits and Systems for Video Technology, 14, no. 1 (2004): 58-73.

${ }^{215}$ Cowger, J. Friction Ridge Skin: Comparison and Identification of Fingerprints. CRC Press, 1992; Maceo, op. cit. 216 Thornton, J. "The One-Dissimilarity Doctrine in Fingerprint Identification." International Criminal Police Review, 32, no. 306 (1977): 89-95.

${ }^{217}$ Maceo, op. cit.
} 
different types of distortion. Such research is more common in other disciplines. In the medical field, for example, research has investigated how an expert develops the complex skill of interpreting X-ray images with a high degree of accuracy. ${ }^{218}$ Within the fingerprint field, the limited research has suggested that some assumptions about the causes of distortion have been wrong. ${ }^{219}$

\subsubsection{Recommendations and Best Practices}

Given the importance of judgments that must be made about distortion in latent prints, it is critical that such judgments be documented clearly. As with other interpretive stages, examiners also need to document any modifications that are made after seeing the exemplar. Thus, Recommendations 3.1 and 3.2, described earlier in this chapter, are relevant to distortion. Likewise, steps to minimize cognitive biases are important for judgments regarding discrepancy. Therefore, Recommendation 3.3, that unnecessary information be kept from examiners, is also important in this context.

\subsubsection{Research Needs in Discrepancy Interpretation}

\section{Variability of Distortion}

Examiners must predominantly rely on the knowledge gained during training and subsequent experience to recognize and interpret distortion. Underestimating the variability of distortion may lead to a false exclusion, while overestimating the variability may lead to a false identification. Studies to measure the variability of distortion and the extreme limits of distortion are needed.

\section{Identification and Interpretation of Distortion}

An examiner's ability to identify types of distortion has not been thoroughly studied. Research is needed not only to determine if an examiner's working assumptions regarding the effects and degree of distortion have an empirical basis but also to determine if, or in what circumstances, a misattribution of distortion may lead to an incorrect conclusion. Experiments in which "ground truth" is known to the researchers (see Chapter 2) are appropriate for this purpose.

\section{Contextual Bias}

There has been little research in the fingerprint domain to determine the extent to which contextual information affects the interpretation of dissimilarities. More research is needed to determine when various context effects, such as confirmation bias, may lead to erroneous conclusions.

\subsection{Source Attribution}

Within the ACE-V process, three traditional options have been available to the examiner to signify findings. First, the examiner can conclude that the source of a known print is the source

\footnotetext{
${ }^{218}$ Sowden, P., I. Davies, and P. Roling. "Perceptual Learning of the Detection of Features in X-Ray Images: A Functional Role for Improvements in Adults' Visual Sensitivity?" Journal of Experimental Psychology: Human Perception and Performance, 26, no. 1 (2000): 379-90.

${ }^{219}$ Langenburg, G. "Deposition of Bloody Friction Ridge Impressions.” Journal of Forensic Identification, 58, no. 3 (2008): 355-389.
} 
of the questioned print. In current practice, this identification is a claim that the latent matches the exemplar and that it would not match exemplars from anyone else in the world. Second, the examiner can conclude that the exemplar and the latent do not match and cannot share a common source, which is a categorical exclusion. Finally, the examiner may determine that the corresponding information in the latent and exemplar prints is inadequate to permit a conclusion. In this case, the examiner would state that the comparison was "inconclusive" and would provide no additional information about the chances that the two prints did or did not share a common source. The ultimate decision occurs near the end of the Process Map, at step 720 or 750, when the examiner determines whether the latent print matches the exemplar. 


\subsubsection{Findings from Existing Research}

\section{The Decision Process}

Much has been written about the decision process by which a latent print examiner moves from feature detection and weighting, through a comparison with the exemplar, to reach a decision. ${ }^{220}$ Broadly speaking, an examiner uses an intuitive procedure to arrive at a subjective probability for source attribution. If this probability is high enough, the examiner declares an identification. If it is low enough, the examiner declares an exclusion, and if it is somewhere between these extremes, the examiner makes no inference (inconclusive).

Bayesian decision theory offers a more complete, formal model of the intuitive decisionmaking process. ${ }^{221}$ According to this model, a latent print examiner should consider both the source probability and the costs and benefits (utilities) of correct and incorrect decisions. More specifically, the Bayesian examiner:

(1) Assesses the prior probability that source of the exemplar left the latent print. An examiner who purports to rely solely on the information in the prints rather than the context of the case or the other evidence against this individual would have no reason to distinguish this individual from anyone else on the planet capable of being where the latent print was found. An examiner who considers more details of the case might treat the source of the exemplar as equivalent to a random person drawn from a smaller population of conceivable suspects. Such reasoning leads to the reciprocal of the size of the relevant population as the prior probability.

(2) Assesses the weight of the evidence as a function of the similarities and differences observed between the latent print and the exemplar. Formally, the weight is a likelihood ratio, as discussed below and in Chapter 1. Examiners may arrive at the weight intuitively (using their knowledge and experience) or by consulting data-driven likelihood models. However, these models currently use fewer features than a human examiner would, and they have other limitations.

(3) Computes the posterior probability of the proposition that the source of the exemplar left the latent print by combining the prior probability and the likelihood ratio according to Bayes's rule (see Chapter 1).

(4) Combines the posterior probabilities with the utilities of the possible correct and incorrect decisions to reach and report the optimal decision.

\footnotetext{
${ }^{220}$ For an overview of the decision process and discussion of the philosophy of making individualization decisions, see generally Ashbaugh, 1999, op. cit.; Vanderkolk, J. "Levels of Quality and Quantity in Detail.” Journal of Forensic Identification, 51, no. 5 (2001): 461-68; Kwan, Q. "Inference of Identity of Source." PhD thesis of Criminology, University of California, Berkeley, 1977. For discussion of the probabilistic nature of identification or individualization decisions see Champod, C. "Identification and Individualization." In Wiley Encyclopedia of Forensic Sciences, edited by A. Moenssens and A. Jamieson, vol. 3, pp. 1508-11. J. Wiley \& Sons, 2009.

${ }^{221}$ Biedermann, A., S. Bozza, and F. Taroni. "Decision Theoretic Properties of Forensic Identification: Underlying Logic and Argumentative Implications.” Forensic Science International, 177 (2008): 120.
} 
Scholarship on the interpretation of forensic evidence argues that (1), (3), and (4) are in the exclusive province of the fact-finder (e.g., the jury in a trial) rather than the expert. ${ }^{222}$ In this view, the examiner should report only the weight of the evidence (2), expressed either quantitatively or qualitatively. Today, however, in most jurisdictions, the examiner implicitly takes all the above steps.

Regardless of whether the details of the Bayesian formulation provide an adequate descriptive or normative model of a latent print examination, the analysis is useful in clarifying the factors that, in some manner, must inform an identification decision. The weight of the evidence, the prior probability, the posterior probability, and the utilities of the possible decisions all bear on an examiner's ultimate conclusion, but presenting that conclusion is not inherent in the examiner's role of evaluating the latent print evidence. In particular, step (2) can be carried out via a likelihood ratio or through a less formal assessment that characterizes the Evaluation phase of ACE-V. Having performed this expert function, the examiner then can describe the weight of the latent print evidence to an investigator or fact-finder (see Chapter 6).

\section{Weight of the Evidence}

Evaluation is the phase in a latent print examination during which the examiner assesses the totality of the features compared in the latent print and the exemplar. This can be thought of as the end result or the culmination of the stages described in earlier sections of this chapter. There are many ways for this assessment to occur, two of which are described below.

The ACE-V process attempts to provide the framework from which a transparent threshold for an accurate and repeatable assessment of friction ridge skin can be established. The Evaluation phase refers to the final evaluation of whether the comparison results are sufficient to allow a determination, and if so, whether that determination is an identification or an exclusion. ACE-V does not, however, prescribe how this is done. ${ }^{223}$ Friction ridge examination by human beings is both human-resource intensive and subjective in the way in which the broadly defined ACE-V process is applied. There are no formal thresholds for any sufficiency determinations, and feature selection and weighting are matters of personal judgment. This is not to suggest that experts are poor at any of these tasks. Rather, it is simply to note the absence of objective criteria. Some countries take a less holistic approach and apply a minimum point standard. Although this does reduce subjectivity, it does not give adequate attention to variations in the rarity and clarity of the minutiae. Currently, the traditional ACE-V approach largely lacks validated, transparent thresholds.

The likelihood ratio approach to evidence interpretation attempts to measure the total weight of evidence using information about the frequency with which different features are observed in the population. As explained in Chapter 1, the likelihood ratio is the probability of the data when one hypothesis is true compared to (divided by) the probability of the data when the competing hypothesis is true. Because it focuses attention on the support the data provide for the relevant hypotheses, the likelihood ratio is especially suitable for assessing the contribution of forensic

\footnotetext{
${ }^{222}$ E.g., Aitken, C. and F. Taroni. Statistics and the Evaluation of Evidence for Forensic Scientists. 2nd ed. John Wiley \& Sons, 2004; Kaye, D., D. Bernstein, and J. Mnookin. The New Wigmore, A Treatise on Evidence: Expert Evidence. 2d ed. Aspen Publishers, 2011.

${ }^{223}$ Mnookin, op. cit.
} 
findings in a balanced way. ${ }^{224}$ It applies regardless of the type of forensic evidence considered. ${ }^{225}$ The likelihood ratio invites forensic practitioners to assess the likelihood of the observed findings (the pattern of matching minutiae and other key observations) under the two different hypotheses that are relevant to the fact-finder (namely, that the prints came from the same source or different sources).

In the case of latent print identification, the forensic findings are a set of concordances and dissimilarities (that can be reduced sometimes to a similarity score) of the comparison between the ridge detail features from the latent print and from an exemplar print from a potential source. The propositions under which the forensic findings are evaluated reflect the issues at hand; for example, either the friction ridge skin area of a potential source has truly left the latent print, or someone else from a relevant population of potential donors is actually the source of the latent print. Assessing the strength of evidence means answering two questions: (1) What is the probability of observing the similarities and dissimilarities if the two impressions come from the same source? (2) What is the probability of observing the similarities and dissimilarities if the two impressions come from different sources?

The "likelihoods" that form the numerator and denominator of the likelihood ratio can represent an examiner's subjective probabilities, or they can be determined from a data-driven probabilistic model. Without becoming mired in details, one can consider the conceptual steps involved in the formal modeling. To answer question (1), the similarity score of the latent print and the known exemplar images are positioned among all the possibilities of comparisons between the exemplar and distorted images of that precise source. Estimates of the distribution of similarity scores for distorted prints might be obtained either empirically (if sufficient control prints are available for that task) or by using a mathematical distortion model with parameters obtained from a large collection of prints taken from an individual. The location of the observed score within the estimated distribution indicates the probability of observing the level of agreement found between the latent print and the known exemplar, given the possibilities of distortion and the hypothesis that they come from the same source (the numerator).

The denominator requires an estimate of the distribution of similarity scores when pairs of prints are drawn from different sources. The variation should be larger than that due to distortion alone, and the level of similarity should be low. The relevant question is whether the similarity score is more consistent with the type of variation that would be expected if the print originated from the same source than if it originated from another source. The next chapter reviews research on methods for automating the assessment of the likelihood ratio.

One final topic associated with the likelihood ratio deserves attention. This concerns the interpretation of the likelihood ratio when an AFIS database search produces the candidate for comparison. There has been extensive debate, primarily in regard to DNA database trawls, about

\footnotetext{
${ }^{224}$ For a short introduction, see Champod, C. and I. Evett. "Evidence Interpretation: A Logical Approach." In Wiley Encyclopedia of Forensic Sciences, edited by A. Moenssens and A. Jamieson, vol. 2, pp. 968-76. J. Wiley \& Sons, 2009.

${ }^{225}$ Aitken, op.cit.; Kaye, Bernstein, and Mnookin, op. cit. The LR framework has been recommended in a standard adopted by all laboratories affiliated with the Association of Forensic Science Providers. Association of Forensic Science Providers. "Standards for the Formulation of Evaluative Forensic Science Expert Opinion." Science and Justice, 49, no. 3 (2009): 161-64.
} 
how the search process affects the likelihood ratio. Most scholarship suggests that producing the suspect through a database search lowers the prior probability and raises the likelihood ratio. ${ }^{226}$ It lowers the prior because there normally was no stronger reason to suspect the subject of the "hit" than there was to suspect other persons in the database. ${ }^{230}$ It raises the likelihood ratio primarily by eliminating (or least reducing the source probabilities for) all other individuals represented in the database. ${ }^{231}$ How to present the results of a database search most fairly remains debatable, but the concerns raised earlier about exemplars resulting from AFIS searches would be handled differently if the examiner were presenting both a prior probability and a likelihood ratio instead of collapsing these quantities into a subjective source attribution.

\section{Knowledge of Examiner Performance}

It can be difficult to create realistic scenarios in which examiners re-examine the same set of prints or in which they examine a set of prints for which the correct conclusion is known. As a result, controlled experiments that would illuminate the actual decisionmaking and source-attribution processes of latent print examiners during casework are unusual. The limited studies that have been done, however, suggest that variation in decisions occur, especially when contextual information is varied. Two noteworthy studies are discussed in Box 3.1.

These experiments pose a real challenge. Experience is of enormous value and allows experts in a range of fields to

\section{Box 3.1: Studies on the Effect of Biasing Information}

In two studies in 2006, researchers collected casework that had been analyzed by experienced latent print analysts during their normal routine work several years earlier. ${ }^{227}$ The laboratory presented this casework to the analysts again in the ordinary flow of casework. In the first study, five examiners received a latent print and exemplar that they had previously classified as a match along with new contextual information that very strongly suggested that the pair did not match. ${ }^{228}$ Three of the five changed from their earlier conclusion of an identification to an exclusion. Another found the evidence inconclusive. ${ }^{229}$

In the second study, six analysts received eight pairs of prints from earlier casework that was a mix of exclusion and individualization decisions. This time, the biasing information was weaker, and a control group that received no biasing contextual information was present. Only two of the six examiners made consistent decisions across all eight pairs of prints; the others changed one or more of the earlier decisions. Of the forty-eight presentations, there were six changes from the earlier judgments. In the biasing condition, there were three changes from individualization to exclusion, and one from individualization to inconclusive. In the control group of examiners who were not given any biasing contextual information, there was one change from individualization to exclusion and one from exclusion to individualization. The authors interpreted these outcomes as establishing "that fingerprint experts were vulnerable to biasing information when they were presented within relatively routine day-to-day contexts, such as corroborative (or conflicting) evidence of confession to the crime." operate at a high level. At the same time, experts can be prone to context effects. Although

\footnotetext{
${ }^{226}$ For a review of the DNA literature, see Kaye, D. "Rounding Up the Usual Suspects: A Legal and Logical Analysis of DNA Database Trawls." North Carolina Law Review, 87, no. 2 (2009): 425-503.

${ }^{227}$ Dror and Charlton, op. cit.; Dror, Charlton, and Peron, op. cit.

${ }^{228}$ The experts were told that the prints were from the highly publicized misidentification of Brandon Mayfield in the Madrid train bombing case.

${ }^{229}$ The study had no control group that unknowingly revisited earlier, challenging pairs of prints that they had determined to match.

${ }^{230}$ Kaye, op. cit., pp. $463-464$.

${ }^{231}$ Ibid., pp. $461-462$.
}

71 Latent Print Examination and Human Factors: Improving the Practice through a Systems Approach The Report of the Expert Working Group on Human Factors in Latent Print Analysis 
additional study is clearly required, the evidence to date in the psychological literature, including the limited number of studies specific to latent print examination, supports Recommendation 3.3, which states that analysts should not be given contextual information that is not directly relevant to their analysis.

\subsubsection{Recommendations and Best Practices}

The discussion of the decision process, the way in which the weight of evidence is determined, and the possibility of errors all argue strongly that the common practice of claiming to uniquely identify an individual is not appropriate. As indicated above, a fingerprint identification was traditionally considered an "individualization," meaning that the latent print was considered identified to one finger of a specific individual as opposed to every other potential source in the universe. However, the recent attention focused on this issue reveals that this definition needlessly claims too much, is not adequately established by fundamental research, and is impossible to validate solely on the basis of experience. Nor does fingerprint evidence have objective standards or a well-validated statistical model that can provide an objective measure of the strength of the fingerprint evidence in a given instance. Therefore, examiners should not claim to be able to exclude every other finger in the world as a potential source. Rather, an identification decision suggests a substantial enough similarity that the examiner believes that the two impressions originated from a common source. But whether any other finger in the world might also be able to leave an impression with a comparable amount of similarity is not fully known, and the examiner's testimony should not suggest otherwise. Regardless of the specific words used to describe an identification, examiners should refrain from claiming that an identification means that they have excluded all other individuals in the world.

\section{Recommendation 3.7: Because empirical evidence and statistical reasoning do not support a source attribution to the exclusion of all other individuals in the world, latent print examiners should not report or testify, directly or by implication, to a source attribution to the exclusion of all others in the world.}

As explained in Chapter 1, the Scientific Working Group on Friction Ridge Analysis, Study and Technology (SWGFAST) recently drafted language that eliminates this needlessly strong meaning of an identification (although SWGFAST also elected to continue to use the term "individualization" notwithstanding its long association with this idea). The proposed SWGFAST draft states, "Individualization of an impression to one source is the decision that the likelihood the impression was made by another (different) source is so remote that it is considered as a practical impossibility." 232

Eliminating the previous "to the exclusion of all others" language from the definition is a step in the right direction. However, the new text raises two concerns. First, it is unnecessary for an examiner to reach conclusions about what is a "practical impossibility." Although fingerprints are clearly highly discriminating, until further research permits better validated statements about probabilities, neither experience nor statistical modeling justifies conclusions about what is or is not practically possible across a population of billions of individuals (and billions times billions

\footnotetext{
${ }^{232}$ Scientific Working Group on Friction Ridge Analysis, Study and Technology. Draft for Comment: Standards for Examining Friction Ridge Impressions and Resulting Conclusions (To Replace: Friction Ridge Examination Methodology for Latent Print Examiners, Version 1.01, and Standards for Conclusions, Version 1.0). Version 1.0. Scientific Working Group on Friction Ridge Analysis, Study and Technology, August 2010, p. 4.
} 
of possible pairs of prints). Second, given that the word "individualization" has been associated precisely with the "to the exclusion of all others" claim of universal individualization based on a premise of general uniqueness (see Chapter 1), it is potentially problematic and confusing to attempt to redefine it by fiat. Using alternative terminology might be a superior solution to attempting to "legislate" a new and slightly modified meaning to a much-criticized term and theory.

In July 2010, the membership of the International Association for Identification (IAI) adopted Resolution 2010-18, allowing members to give qualified opinions if supported with a statistical model "accepted as valid by the IAI." ${ }^{233}$ However, the IAI also stated that it does not endorse any models as ready for casework or introduction into the courtroom. The resolution is another important step in the right direction, and the Working Group supports further movement in this direction.

Until statistical models are implemented, concerns remain about over-expressing the evidence (see Chapter 6). In addition, there are legitimate concerns about under-expressing, or not expressing at all, the wealth of evidence that can be found in the large grey zone between "identification" and "exclusion." In the future, it seems likely that examiners will be required to provide more nuanced information than the current, narrow range of allowable conclusions. In time, it should be possible to provide quantitative measures in the form of likelihood ratios or posterior probabilities (see Chapters 4 and 6). Even if this does not happen, qualified conclusions consistent with existing knowledge should be permitted.

The difficult problem here is to find a balance between the two views of how to report fingerprint evidence, since both views have strengths and weaknesses. If absolute conclusions are not allowed, then one is left only with reported conclusions that allow for more than one possible donor of a latent print, as is the case with other types of forensic evidence canvassed in Chapter 6. Conversely, there may be instances in which a single source attribution is warranted based upon the theory of local uniqueness outlined in Chapter 1. If qualified opinions are not allowed, then one may be keeping from fact-finders evidence that exists in the grey continuum between absolute source attribution and absolute exclusion. There is often evidence that is strongly associative to a source, based upon high likelihood ratios (either explicitly or implicitly derived), but that does not rise to the level at which an examiner feels comfortable providing a traditional "identification" conclusion. This evidence and the decision to withhold it are typically not communicated to the fact-finders. This all-or-nothing approach keeps potentially critical evidence from fact-finders based on the profession's need to maintain categorical opinions of "identification" or "exclusion." Alternatively, there may be instances (for example, when dealing with fully rolled fingerprints, identification of deceased individuals, and ten-print examinations) when categorical decisions such as "identification" or "exclusion" are completely warranted, justified by the overwhelming availability of discriminating features, and practical for the circumstances. Finding the balance between these issues is not easy, especially in the absence of data showing which approach is most appropriate for the circumstances and which approach will not confuse or mislead the fact-finders.

${ }^{233}$ International Association for Identification. IAI Resolution 2010-18. International Association for Identification, July 16, 2010. 
The Working Group could not resolve the important questions surrounding whether, or when, latent print examiners should be permitted to testify to probabilities rather than absolute categories, nor did it endeavor to determine what form probabilistic testimony should take. These issues will continue to grow in both salience and importance in the coming years. Hence, the Working Group strongly recommends ongoing attention to this issue.

\section{Recommendation 3.8: The friction ridge examiner community and other experts should determine under what circumstances a qualified rather than an absolute conclusion is warranted.}

\subsubsection{Research Needs in Source Attribution}

Research in the latent print domain in past decades has largely been centered on automated fingerprint identification systems and physical and chemical detection techniques. Very little research, until the last few years, has centered on human performance in pattern recognition and interpretation. Much more research is needed not only to validate ideas being put forward today but also to expand understanding further.

- Research and testing to develop greater knowledge about error rates for latent print examination must be undertaken. While a single error rate for the field is neither desirable nor achievable, it is critical to develop more knowledge about error rates, what affects them, and the extent to which they are correlated to the relative difficulty of comparisons (see Chapter 2). Indeed, it is possible that such work will help define levels of complexity.

- It will be important to understand what influences affect not only the ultimate decisions of examiners but also their decision-making thresholds. It is entirely possible that bias influences decision thresholds, but not decision outcomes, in easy latent print comparisons. At what point does this impact on thresholds change outcomes?

- With expanded knowledge on thresholds must come research that investigates whether considering certain features makes examiners more vulnerable to inaccurate decisions.

- More research is needed into the relationship between risk and cost to examiners when making decisions. For example, does the cultural default position of law enforcement and its relationship with forensic practitioners create an environment in which latent print examiners shun the inconclusiveness of evidence in favor of more definitive conclusions that are more conducive to current law enforcement expectations? Research will better inform whether there needs to be a change in the culture of forensic support services in relation to their policing partners.

- The confidence associated with decision making in latent print examination should be studied to see if confidence levels are consistent when making identifications as opposed to exclusions. It will also be important to determine under what circumstances a qualified conclusion would be warranted, whereby a lack of strong confidence in a particular decision based upon a latent print comparison might be better expressed as "consistent with." In other words, when might it be appropriate to offer a conclusion that is less emphatic? 


\subsection{The Need for Research}

The previous sections each ended with a list of research topics relevant to the particular interpretative stage. This section characterizes in broad terms the types of research that are required and incorporates a final recommendation about the importance of the federal government finding a mechanism to fund a research program.

The research topics identified throughout the chapter can be broadly categorized into three types: (1) developing metrics or measurements that characterize key elements of the interpretive process, (2) assessing the reliability of measures or determinations, and (3) determining the factors associated with different performance levels.

\subsubsection{Developing Metrics}

Across the interpretive stages, there is a need to carefully define elements being used by examiners in a manner that allows the processes to be studied quantitatively. Examples include developing a way to characterize selected features (perhaps by location and degree of confidence), developing a series of indicators of sufficiency, conducting additional work on the frequency of features in different populations, and perhaps ultimately developing a measure of the complexity of a particular comparison.

\subsubsection{Assessing Reliability, Reproducibility, and Validity}

A process for making measurements or decisions is reliable when the outcomes are repeatable and reproducible, both within and across examiners. It is important that feature selection, sufficiency determination, feature weighting, judgments regarding distortion, and ultimate decisions be reproducible. An examiner repeatedly presented with the same sets of prints should obtain the same interpretive conclusions (intra-examiner reliability), and different examiners also should be able to obtain the same results (inter-examiner reproducibility). For many, these two concepts are at the heart of the ongoing discussion about the desirability of research to reaffirm the consistency of latent print judgments. Some research has been conducted on this issue, ${ }^{234}$ but more is needed.

Accuracy is whether the determinations made by the analyst correctly express the true state of the world. This accuracy can be measured in various ways, as discussed in Chapter 2. A final term, validity, also refers to accuracy but extends further to encompass the issue of whether the measurements, judgments, and decisions being made are appropriate for their common uses. Thus, validity is a relative term. The polygraph is a valid means of detecting stress, but its validity as a device to detect conscious deception is much more doubtful. Whether ACE-V does what its practitioners claim it does and whether the resulting decisions are adequately supported by scientific knowledge are questions about validity.

\footnotetext{
${ }^{234}$ Langenburg, G. "A Performance Study of the ACE-V Process: A Pilot Study to Measure the Accuracy, Precision, Reproducibility, Repeatability, and Biasability of Conclusions Resulting from the ACE-V Process." Journal of Forensic Identification, 59, no. 2 (2009): 219-57; Evett and Williams, op. cit.; Dror and Charlton, op. cit.
} 


\subsubsection{Explaining Variations in Performance}

The final element of a research program would attempt to explain observed variations in reliability or accuracy. A key question that underlies much of the discussion in this chapter is how the complexity of a comparison impacts the reliability of the determined conclusions. There are, however, as many types of analyses as one can imagine. Relating performance to the individual characteristics of the examiner, including physical characteristics, training completed, and aspects of the working environment, may help supervisors determine strategies for recruitment and training to optimize performance. This list is not comprehensive. It argues strongly, however, as do other chapters in this report, for an extensive research program.

\section{Recommendation 3.9: The federal government should support a research program that aims to:}

a. Develop measures and metrics relevant to the analysis of latent prints;

b. Use such metrics to assess the reproducibility, reliability, and validity of various interpretive stages of latent print analysis; and

c. Identify key factors related to variations in performance of latent print examiners during the interpretation process.

\subsection{Summary}

For each interpretive stage of latent print examination, this chapter offers recommendations and best practices to help ensure the accuracy and transparency of the interpretive processes. The chapter describes and defines the multiple critical interpretive stages that are part of every latent print comparison. The Working Group based its analysis and recommendations on the current state of knowledge established by published research regarding the interpretive stages and on the implications of this research for potential concerns and pitfalls that may arise in the interpretive process. 


\section{Chapter 4: Looking Ahead to Emerging and Improving Technology}

\section{Introduction and Scope}

Latent print examiners use online databases, digital enhancement software, and other types of technology to assist with the Analysis, Comparison, Examination, and Verification (ACE-V) process. Combining these tools with the examiner's own expertise can make investigations more reliable and easier to explain to juries. This chapter discusses how these and newer technologies can automate complicated and work-intensive parts of the process and thereby reduce human error. In particular, the chapter addresses the following technology-related questions:

- $\quad$ Fingerprint and palm print databases at the federal, state, and local levels can be searched through automated fingerprint identification system (AFIS) databases, which saves examiners from manually searching through hundreds of exemplars looking for close matches. How can these systems (and the training provided to examiners in their use) be improved to provide greater confidence in the results and more value to examiners?

- Digital scanning devices known as livescan are frequently used to capture exemplar prints and to submit them to automated systems. This process allows high-quality digital images to be stored in central databases. How can this technology be improved?

- When comparing digital exemplars to digital latent prints, examiners can enhance the images to clarify minute details. What are the best practices for enhancement? The process of enhancement can be challenged in court, so examiners must understand and be able to explain the technology.

- When testifying, examiners should qualify their conclusions instead of stating an exclusion or identification in absolute terms. With modern computational power, researchers have implemented various probabilistic models to allow examiners to quantify the similarities between two prints and to assess the implications of these similarities. How can these models be used, and what are their long-term implications?

- How can automated systems assist in ascertaining the quality and quantity of the features in a latent print?

- How do examiners interact with new technological tools? How can technology-training programs be more effective for examiners at all career levels?

\subsection{Automated Fingerprint Identification Systems}

AFIS databases exist at federal, state, and local levels. ${ }^{235}$ The Integrated Automated Fingerprint Identification System (IAFIS), managed by the Federal Bureau of Investigation (FBI), holds most of the fingerprint sets and other identification records collected in the country, both from criminal and civil sources. In addition, every state either has its own AFIS or shares an AFIS with other states. Many localities, especially large metropolitan areas, have their own systems as

${ }^{235}$ See generally Moses, K. "Automated Fingerprint Identification System (AFIS).” The Fingerprint Sourcebook. National Institute of Justice, 2011. 
well. All of these automated systems have capabilities such as latent print searching, electronic image storage, and fingerprint image transmission, and some include palm prints as well. ${ }^{236} \mathrm{In}$ criminal cases, IAFIS searches through millions of criminal entries and returns results in about 10 minutes. ${ }^{237}$

\subsubsection{Training}

The companies that supply AFIS software and hardware provide general training on using the system, but they do not clearly define which latent prints are most suitable for database searches. With experience, latent print examiners may develop an understanding of what makes a latent print of "AFIS quality" for a particular system, but receiving formal instruction and training in making these determinations would be more efficient.

Likewise, AFIS vendors typically do not provide training on how to encode a latent print to maximize the match capabilities of the system. An AFIS search merely provides a list of exemplars with the highest similarity scores, as determined by a proprietary algorithm. Latent print examiners often use trial and error, entering the same latent print multiple ways to see the effect on the resulting candidate list. Vendors should expand their training programs to include instruction in the most effective methods for encoding.

In addition, more education on the meaning of the AFIS scores could be useful. Some examiners use rank or score as a filter, hoping to avoid wasting time on fruitless comparisons. Thus, many examiners will not look beyond rank 5 or 10 (sometimes by policy). Some examiners use a system-specific score as a cutoff. Others look at candidates based upon the differences between scores, believing that any score that is substantially greater than that for the next candidate is worth comparing, whereas a series of candidates with very similar scores are much less likely to be matches. Some examiners look at all the candidates, assigning some weight to a high AFIS score or rank. Still other examiners entirely disregard score and rank information. There is some evidence that knowing the rank of an AFIS candidate influences the judgments of some examiners, but it is not clear whether this knowledge benefits or hinders the ACE-V process. ${ }^{238}$

On the basis of the available research, we can make no recommendation on whether the examiner should be blinded to the scores or ranks of AFIS-generated exemplars when conducting comparisons and evaluations. Regardless of the advisability of knowing the scores or ranks of these exemplars, however, latent print examiners who frequently work with exemplars from AFIS searches should have a clear understanding of how the system works and the meaning and limitations of AFIS scores and ranks.

\footnotetext{
${ }^{236}$ Ibid.

${ }^{237}$ Federal Bureau of Investigation. "Integrated Automated Fingerprint Identification System.” Accessed November 25, 2011. http://www.fbi.gov/about-us/cjis/fingerprints_biometrics/iafis/iafis.

${ }^{238}$ See Dror, I., K. Wertheim, P. Fraser-Mackenzie, et al. "The Impact of Human-Technology Cooperation and Distributed Cognition in Forensic Science: Biasing Effects of AFIS Contextual Information on Human Experts." Journal of Forensic Sciences (forthcoming).
} 


\subsubsection{AFIS Algorithms}

AFIS algorithms could be expanded to match prints to account for the diagnostic value of the positions at which minutiae are located. Exploiting this information might improve AFIS searches, and research into the utility of using the additional information might assist in the development of probabilistic models (as discussed in section 4.3.1).

\subsubsection{Interoperability}

The 2009 report of the National Research Council ${ }^{239}$ recommends improving the interoperability of AFIS systems among states and the FBI. Searching multiple databases would help states identify criminals who have been fingerprinted in other states. Because the National Institute of Standards and Technology has established a separate working group on this topic, this Working Group did not pursue this issue.

\subsubsection{Other Skin Surfaces}

Automated search technology can be applied to impressions from all friction ridge skin areas, such as palmar (palm or digital joint) or plantar (toe or foot) friction ridges. These impressions sometimes are searched if the capability exists within the automated identification system of the investigating department. Nonetheless, exemplars from these areas are collected less frequently, and it may prove difficult to construct large databases for these impressions.

Recommendation 4.1: The federal government should support research programs to improve automated fingerprint identification systems. Such programs could address the following issues:

a. Expanding the algorithms used to match prints to account for the fact that the diagnostic value of minutiae depends on the region in which they are located;

b. Making fingerprint and palm print databases interoperable among local, state, and federal automated identification systems; and

c. Increasing compatibility between automated identification systems and other latent print software tools, including digital enhancement programs, probability calculation programs, and automated quality assessment programs.

\subsection{Digital Images}

\subsubsection{Livescan Systems}

Police departments (and other government agencies) traditionally recorded known exemplars by inking a person's ten fingers and rolling them onto a paper ten-print card. Larger departments now use livescan technology to digitally capture finger and palm prints. ${ }^{240}$ This inkless method captures digital images of exemplar prints, which can be transmitted together with demographic data and mug shots to a central location. The system rejects the print if the quality falls below a

\footnotetext{
${ }^{239}$ National Academy of Sciences, National Research Council, Committee on Identifying the Needs of the Forensic Science Community. Strengthening Forensic Science in the United States: A Path Forward. National Academies Press, 2009.

${ }^{240}$ See e.g., Moses, op. cit.
} 
certain threshold. When properly maintained and used by adequately trained personnel, livescan can improve both the quality and speed of the ten-print collection process.

However, livescan technology can be improved. For example, livescan can produce distorted images of the flow of friction ridge skin because of the process of recording a three-dimensional object on a two-dimensional surface. Some livescan (and almost all automated fingerprint identification) systems compress images. This practice causes critical details in the friction ridge impression to be lost, even when the images are decompressed. Most AFIS and livescan systems use images scanned at 500 pixels per inch. Although this standard satisfies the FBI's Electronic Fingerprint Transmission Specification standard, such images, especially if highly compressed, may not capture all Level 3 Detail (L3D) that would be desirable for examiners performing comparisons. Because of these limitations, many examiners prefer inked prints over livescan images for final comparisons.

\subsubsection{Digital Enhancements}

The use of digital processing and enhancement is now routine in latent print analysis. If properly used to remove background distractions that are not part of the friction ridge detail, digital enhancement can improve both the examiner's ability to analyze the image and the jury's ability to understand the evidence. Yet filtering an image also could create artifacts that an examiner might mistake for minutiae. Figures 4.1 and 4.2 provide examples of applying filters to digital images.

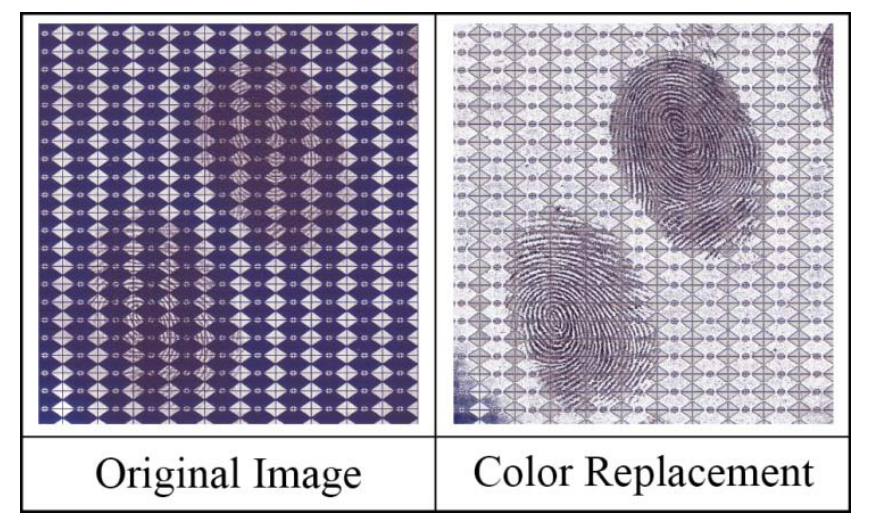

Figure 4.1: Example of a color replacement filter to remove color from a playing card $^{241}$

\footnotetext{
${ }^{241}$ Images reproduced and adapted courtesy of Robert Garrett, past president and chairman of the board of the International Association for Identification.
} 


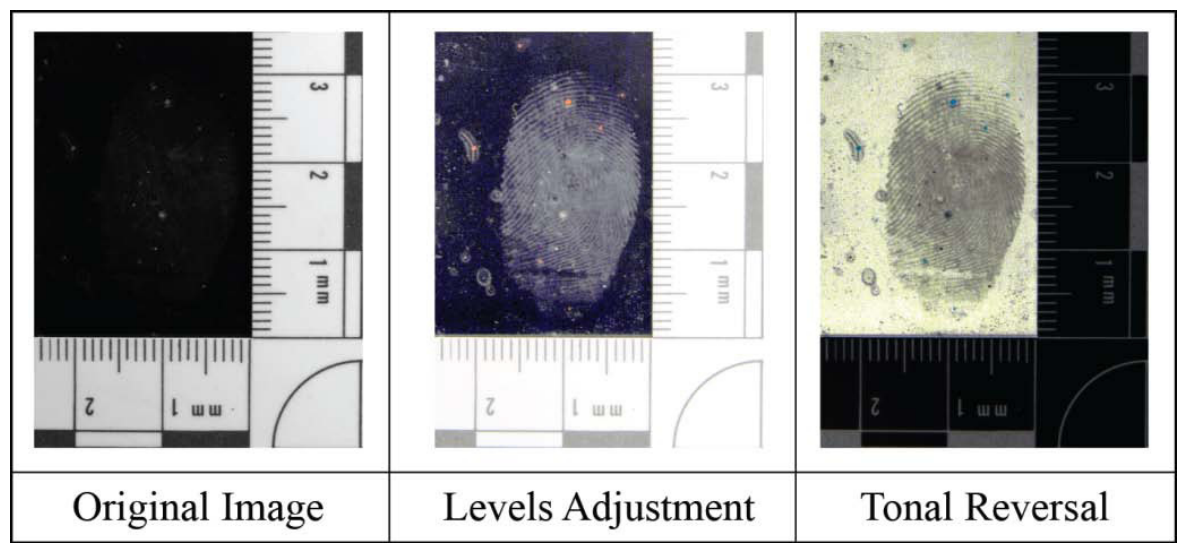

Figure 4.2: Example of filters used to adjust color levels and to reverse the colors ${ }^{242}$

Because of the possibility of artifacts, judges should not unquestioningly accept an expert's claim that an enhanced image is accurate. There is a growing judicial recognition that the party introducing enhanced images as trial exhibits must be able to defend the enhancements in each case. ${ }^{243}$ In State v. Swinton, ${ }^{244}$ the Connecticut Supreme Court upheld the admission of a photograph of a digitally enhanced bite mark; however, the court rejected the prosecution's argument that the court should accept the enhancement as easily as the original photograph. The court accepted the enhancement because the expert testified "in specific detail as to the process" of programming the enhancement software. Moreover, the court rejected overlays created with Adobe Photoshop because the witness could not explain the use of the program for that purpose. The California Supreme Court issued a similar opinion in People v. McWhorter. ${ }^{245}$ In that case, a defense expert prepared an enhanced image of a window. In upholding the exclusion of the testimony, the court noted that the expert "could not identify the computer program he used to enhance or 'electronically emboss' the image in question, nor could he satisfactorily explain the full nature of the process he used to create it." 246

Although Swinton and McWhorter may have insisted on more thorough foundations than are typical, the decisions highlight the importance of clearly understanding the process and benefits of digitally enhancing images. Under the usual rules for scientific evidence and for authenticating photographs, an expert must be able to demonstrate that the software can accurately enhance the original image and that it was used properly.

The Scientific Working Group on Imaging Technology (SWGIT) has proposed best practices for the acquisition, storage, and processing of images. ${ }^{247}$ Underlying the guidelines are several basic principles. In particular, forensic service providers should validate latent print enhancement technologies prior to use in casework. To maintain an appropriate audit trail, the providers

\footnotetext{
${ }^{242}$ Images reproduced and adapted courtesy of Robert Garrett, past president and chairman of the board of the International Association for Identification.

${ }^{243}$ State v. Hartman, 754 N.E.2d 1150 (Ohio 2001).

${ }^{244}$ State v. Swinton, 847 A.2d 921 (Conn. 2004).

245212 P.3d 692 (Cal. 2009).

${ }^{246}$ Ibid, 726.

${ }^{247}$ See International Association for Identification: Scientific Working Group on Imaging Technology (SWGIT). Accessed April 23, 2011. http://www.theiai.org/guidelines/swgit/index.php.
} 
should retain the unenhanced image and should track any digital enhancements made to subsequent copies. Examiners should make it clear in their documentation and testimony that an image has been enhanced. If the proffered image is enhanced, the proponent should make the earlier versions of the image available to the opposition before trial. If the examiner cropped the image, the deleted material should be available for inspection. The proponent should not be permitted to claim that the original, unaltered image is unavailable due to the enhancement software that was used.

In many cases, examiners employ enhancement techniques during their analyses but submit the unaltered images to the court as evidence. ${ }^{248}$ Forensic service providers should ensure every examiner has received training in the technology used to assist in analysis. Moreover, the provider should announce guidelines for the use of such technology. Forensic services providers should follow the best practices the proposed by SWGIT for the acquisition, storage, and processing of images. Developers should validate enhancement technologies used in latent print processing. The laboratory using the technology should validate the technology in its work environment and should retain the original, unenhanced image.

\subsection{Probabilistic Models to Assess Latent Print Evidence}

As discussed in Chapters 3 and 6, examiners often offer opinions as to the source of the latent print in absolute terms. Indeed, as noted in Chapter 6, a 1979 resolution of the International Association for Identification announced that it was professional misconduct for an examiner to use the term "probable" in a final opinion. ${ }^{249}$ Although Resolution 2010-18 declares that the prior resolution was "not consistent with advancements," 250 most courts still allow the examiner to opine in absolute terms and to attribute a particular fingerprint impression to a single individual's finger to the exclusion of all other persons in the world. These courts do not demand that the examiner quantify the extent of any uncertainty (see Chapter 6).

However, Chapter 3 recommends against testimony in this form. There is always some non-zero probability that some skin other than the defendant's produced the latent print. Even when this probability is so small that a source attribution is warranted, the opinion would be more precise and complete if the examiner could provide an estimate of this probability. Models to allow and support this qualified response are being developed and validated.

\subsubsection{Probabilistic Models}

The last 100 years have seen many efforts to characterize the significance of similarities between pairs of fingerprints. Some models have tried to demonstrate the discriminating power of latent

\footnotetext{
${ }^{248}$ Annotation, Admissibility and Weight of Fingerprint Evidence Obtained or Visualized by Chemical, Laser, and Digitally Enhanced Imaging Processes, ALR 5 th Alert, 110 (2003): 213.

${ }^{249}$ International Association for Identification. "Resolution VII." Identification News, 29 (August 1979).

${ }^{250}$ International Association for Identification. IAI Resolution 2010-18. International Association for Identification, July 16, 2010.
} 
print evidence, other models have aimed at proving the uniqueness of latent print impressions, and still others have focused on quantifying the weight of the latent print evidence in the case. ${ }^{251}$

Probability models date back to Galton's 1892 model. ${ }^{252}$ Modern efforts to determine the probability of correct latent print identification are based upon determining the locations of minutiae, ${ }^{253}$ of minutiae and ridges, ${ }^{254}$ and even of pores $^{255}$ (see Figure 4.3). These models incorporate measures of tolerance in accounting for skin distortion and the degradation of crime scene impressions. To date, research into probability models has been limited to the distal segments of the fingers; the remainder of the friction ridge skin has not been considered. By estimating the probability of random correspondence for any given set of latent print features rather than the probability of all of the features of a complete exemplar, the calculations reflect the quantity of information present in the latent fingerprint.

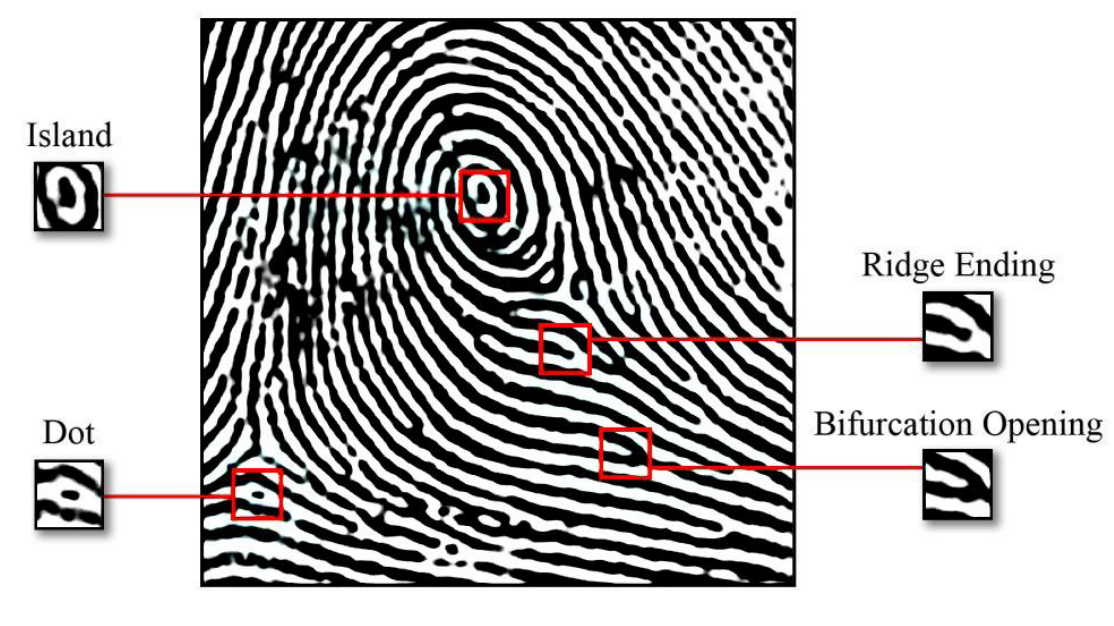

Figure 4.3: An example of some minutiae locations in a fingerprint ${ }^{256}$

Although the modern models represent a major improvement over the early efforts, most models assume independence among minutiae. ${ }^{257}$ That is, they assume that the occurrence of one minutia has no relationship to the occurrence of any other set of minutiae. However, minutiae might not be independent. Some data (see Chapter 3 ) indicate that the frequency of certain

${ }^{251}$ Stoney, D. "Measurement of Fingerprint Individuality." In Advances in Fingerprint Technology, edited by Henry C. Lee and R.E. Gaensslen, 327-87. 2nd ed. CRC Press, 2001; Langenburg, G. "Scientific Research in the Forensic Discipline of Friction Ridge Individualization.” In The Fingerprint Sourcebook. National Institute of Justice, 2011.

${ }^{252}$ Galton, F. Finger Prints. 1892. Reprint, William S. Hein \& Co., 2003.

${ }^{253}$ Dass, S., Y. Zhu, and A. Jain. "Statistical Models for Assessing the Individuality of Fingerprints." Proceedings. Fourth IEEE Workshop on Automatic Identification Advanced Technology, 2005.

${ }^{254}$ Chen, Y. and A. Jain. "Beyond Minutiae: A Fingerprint Individuality Model with Pattern, Ridge, and Pore Features." In Proceedings, International Conference on Biometrics (ICB), Advances in Biometrics, Third

International Conference, Alghero, Italy, June 2-5, 2009; Su, C. and S. Srihari. "Evaluation of Rarity of Fingerprints in Forensics." In Proceedings of Neural Information Processing Systems Conference, Vancouver, Canada, December 2010.

${ }^{255}$ Chen and Jain, op. cit.

${ }^{256}$ Fingerprint image adapted from the Royal Canadian Mounted Police. Fingerprint Manual. Chapter 2. Royal Canadian Mounted Police, 1990.

${ }^{257}$ Pankanti, S., S. Prabhakar, and A. Jain. "On the Individuality of Fingerprints.” IEEE Transactions on Pattern Analysis and Machine Intelligence, 24, no. 8 (2002): 1010. 
minutiae varies depending on the location of the minutiae within the larger pattern. One study reports that in 100,000 randomly chosen fingerprints of exemplar quality, there is only a 10-14 probability that some pair of them will match in regard to both minutiae and ridge shape. ${ }^{258}$ Without the assumption of independence, however, the computed probability could be orders of magnitude higher. ${ }^{259}$

\section{Likelihood Ratio Models}

Some scientists have developed models that provide likelihood ratios in biometric verification ${ }^{260}$ and in forensic friction ridge analysis. ${ }^{261}$ The likelihood ratio combines information on the similarity and rarity of features in a pair of prints. In one approach, the initial step is to quantitatively compare an appropriate set of features characterizing Level 1 Detail (L1D) such as ridge flow, Level 2 Detail (L2D) such as minutiae, and minute Level 3 Detail (L3D) details such as pores between the two fingerprint images. This process of marking features can be entirely manual or partly or fully automated. If a single number is used as an overall measure of similarity and rarity, its probability distributions in two populations can be considered. One probability distribution comes from a large number of images derived from the same finger; the other comes from a sample of images derived from the fingers of different individuals. The likelihood ratio indicates how many times more probable it is to find characteristics with the overall similarity score when the known exemplar and the latent come from the same finger than when they come from different fingers. (For further explanation of likelihood ratios, see Chapters 1, 3, and 6.)

Studies of likelihood ratio models have produced at least three important findings. ${ }^{262}$ First, when fingerprint impressions come from the same source, they display a large number of matching features, and the likelihood ratio is large. Second, same-source impressions infrequently yield likelihood ratios less than one when the two impressions have the same origin. Conversely, likelihood ratios greater than one seldom appear when the two prints have different origins. Thus, research with data sets in which ground truth is known demonstrates that the models usually point to the correct conclusion. In addition, these data sets can be used to estimate error rates for making all decisions based strictly upon a particular threshold for the likelihood ratio. Finding much larger likelihood ratios for impressions from the same source than for impressions from different sources supports the claim that fingerprints contain highly discriminating

\footnotetext{
${ }^{258} \mathrm{Su}, \mathrm{C}$. and S. Srihari. "Generative Models for Fingerprint Individuality Using Ridge Models." In Proceedings $19^{\text {th }}$ International Conference on Pattern Recognition, Tampa, Florida, December 8-11, 2008.

${ }^{259}$ Recent work relaxing the independence assumption is $\mathrm{Su}$ and Srihari, 2010, op. cit.

${ }^{260}$ Bazen, A. and R. Veldhuyis. "Likelihood-Ratio-Based Biometric Verification." In IEEE Transactions on Circuits and Systems Video Technology, 14 (2004): 85; Gonzalez-Rodriguez, J., J. Fierrez-Aguilar, D. Ramos-Castro, et al. "Bayesian Analysis of Fingerprint, Face and Signature Evidences with Automatic Biometric Systems." Forensic Science International, 155 (2005): 126.

${ }^{261}$ Champod, C. and P. Margot. "Computer Assisted Analysis of Minutiae Occurrences on Fingerprints.” In Proceedings of the International Symposium on Fingerprint Detection and Identification, 1995, 305-18; Neumann, C., C. Champod, R. Puch-Solis, et al. "Computation of Likelihood Ratios in Fingerprint Identification for Configurations of Any Number of Minutiae.” Journal of Forensic Sciences, 52, no. 1 (2007): 54 - 64; Neumann, C., C. Champod, R. Puch-Solis, et al. "Computation of Likelihood Ratios in Fingerprint Identification for Configurations of Three Minutiae.” Journal of Forensic Sciences, 51, (2006): 1255; Egli, N., C. Champod, and P. Margot. "Evidence Evaluation in Fingerprint Comparison and Automated Fingerprint Identification SystemsModelling Within Finger Variability.” Forensic Science International, 167 (2007): 189 - 95.

${ }^{262}$ Champod and Margot, op. cit.; Neumann, Champod, Puch-Solis, et al., 2007, op. cit.; Neumann, Champod, PuchSolis, 2006, op. cit.; Egli, Champod, and Margot, op. cit.
} 
information. Finally, different configurations of the same number of minutiae produce different likelihood ratios, depending on the spatial relationship between the minutiae. In many instances, configurations with few minutiae have larger likelihood ratios than configurations with more minutiae. It follows that a simple count of matching minutiae is an imprecise measure of the similarity of two prints.

\section{Future Probabilistic Models}

Additional work should be done to further demonstrate the reliability and validity of latent print probabilistic models. This work will necessitate studies with large-scale, representative databases. Furthermore, once a model is validated, careful thought must be given to the best method of communicating its output to the actors in the criminal justice system (see Chapter 6).

To facilitate the development of probabilistic models, a research database could be created by removing personal identifiers from digital images in AFIS databases. Anonymous versions of criminal DNA databases have been used for statistical research, ${ }^{263}$ and there is no insuperable legal obstacle to doing the same with fingerprint records in the possession of the government. ${ }^{264}$ The only social or psychological risk to individuals whose prints are in the existing databases is that the de-identified images from a criminal file somehow would permit a researcher to discover who has been arrested for or convicted of unspecified crimes. However, the researchers have no means to link an image with a name, and even if they could do so, that an individual has a criminal conviction normally is not private information. Therefore, AFIS database administrators should take the simple step of de-identifying a large set of digital images for research purposes. This will permit the validation of probabilistic models and will facilitate studies into the frequencies of various fingerprint features (discussed in Chapter 3 ).

\section{Recommendation 4.2: To facilitate the validation of probabilistic models and other statistical research, the federal government should create large, anonymous databases of exemplars and latent prints.}

More generally, with suitable support for modern research, some of the probability models that researchers are currently developing could be added to the examiner's toolkit in the near future. To prepare for such models, the latent print examiner community should formulate workflow guidelines to transition the models from the research setting to the forensic laboratory and should begin training examiners in elementary probability and statistics to enable them to interpret the output of the models. Recommendations 3.5 and 3.9 in Chapter 3, concerning federal support of research and educating examiners about statistical methods, are pertinent here.

\subsubsection{Admissibility}

Once validation studies are published, opinions based upon a probability model could be proffered in court. If opposing counsel objects and challenges the validity of the models, the courts would need to determine the admissibility of the evidence. As discussed in Chapter 6, the leading standards in the United States for making this determination are the scientific validity

\footnotetext{
${ }^{263}$ Kaye, D. "Trawling DNA Databases for Partial Matches: What Is the FBI Afraid Of?" Cornell Journal of Law and Public Policy, 19 (2009): 145.

${ }^{264}$ See 5 U.S.C. $\S 552 \mathrm{a}(\mathrm{b})(5) ; 42$ U.S.C. $\S 3789 \mathrm{~g}(\mathrm{~b})$.
} 
standard of Daubert v. Merrell Dow Pharmaceuticals, Inc. ${ }^{265}$ and the general acceptance standard of Frye v. United States. ${ }^{266}$ Under these standards, the proponent of the output of a statistical model or an examiner's opinion based on this model would have to show that the model is valid or generally accepted in the scientific community. It is premature to predict which probabilistic models will be validated and proven useful in court; the optimal model may prove to be one that has yet to be proposed. It is possible that multiple opinions based upon different probability models as well as the output of the model itself would be admissible.

\subsubsection{Implications of the Validation of a Probabilistic Model}

A useable probabilistic model could significantly affect the methods of latent print examination. First, the model might require new metrics in latent print images, that is, the documentation and measurement of new features. Some of the current models rely on measurements that many examiners do not take, such as the distance between friction ridge features. If measurements and the computation can be automated, then the use of the model could reduce the risk of human error in making and using such measurements.

Additionally, judicial acceptance of testimony based upon a probabilistic model could change the presentation of examiners' opinions in court. Chapters 3 and 6 urge that examiners clearly communicate the qualified nature of an opinion to the court. Reliance on statistical models would enable the examiner to acknowledge the uncertainty of the conclusion or to dispense with a binary opinion.

Lastly, judicial acceptance of probabilistic models would not force examiners to become statisticians, but it would require them to be familiar with certain statistical concepts to testify to probabilities or to an opinion based upon a probabilistic model. Specifically, examiners would need to understand uncertainty, variability, conditional probabilities, probability distributions, and likelihood ratios. They should also be able to explain these concepts to judges and juries. Statisticians and the latent print examiner community can collaborate to design a training program for examiners.

Recommendation 4.3: The latent print examiner community should expand the training of examiners in elementary probability theory to enable examiners to properly utilize the output of probabilistic models.

\subsection{New Technologies for Latent Print Examination}

\subsubsection{Automated Quality Assessment}

Early in the examination process, the examiner must decide whether the latent print is suitable for further analysis - this is the Analysis phase in the ACE-V process. In the experience of the Working Group, this initial quality assessment of the latent print is often the weak link in the analytical process, as examiners could make two types of errors. In the first type of error, the examiner prematurely discards a print that should be deemed suitable for further analysis and

265509 U.S. 579 (1993).

266293 F. 1013 (D.C. Cir. 1923). 
comparison, possibly resulting in a missed identification or exclusion. In the second type of error, the examiner continues to evaluate a latent print that he ought to have deemed unsuitable for further processing. This outcome results in wasted time and might even contribute to an erroneous identification.

Currently available automated systems can assist in various steps within the Analysis phase. Automated systems can measure the distance between certain features in two images. They can also generate probabilities for such distances under the assumptions that the image of the latent print comes from a finger known to have produced an exemplar and that the latent comes from a finger of a randomly selected individual represented in a reference set of exemplars from many individuals. Such conditional probabilities might be used in their own right or to corroborate or contradict the judgment of a human examiner. Thus, an automated technique employing a validated algorithm for judging the quality of an image and its suitability for forensic identification would be extremely useful.

There have been attempts to develop automated quality assessment technology. ${ }^{267}$ Although the efforts have so far met with limited success, ${ }^{268}$ automation seems possible. Given a suitable way to calibrate the extent and clarity of the features in the latent and exemplar prints, an objective quality measure could be calculated. This is not to say that the task will be easy. The challenges to creating an automated quality assessment procedure are threefold: (1) identifying the most critical elements to assess the quality of an image, (2) choosing a metric to assess each element selected, and (3) combining these assessments to determine when the quality is sufficient to warrant further effort. Just as prints vary in clarity and completeness, examiners vary in experience and skill. An impression deemed unsuitable by an examiner of lesser skill might be considered useful by a more seasoned examiner. In addition, if an algorithm indicates that a latent print is suitable, an examiner might feel pressured to go beyond the normal comfort level to reach a definite conclusion. ${ }^{269}$ This pressure may be compounded by the widespread assumption that if a print is judged to be of value, a comparison with an exemplar print should always permit the examiner to either identify or exclude. Furthermore, there is no guarantee that algorithms can be developed to mimic this type of human interpretation, especially the evaluation of L3D, or that examiners can develop an effective strategy for employing such technology.

Ultimately, the research may yield the conclusion that automation cannot improve the initial quality assessment. Any investigation into the possibility of automating this stage ought to be conducted with these risks and challenges in mind. Nevertheless, because the Analysis phase is so important to the overall examination, a concerted effort to automate the quality assessment step, at least partially, is worthwhile.

\footnotetext{
${ }^{267}$ Hicklin, A., et al. "Latent Fingerprint Quality: A Survey of Examiners.” Journal of Forensic Identification, JulAug 2011, 385-417.

${ }^{268}$ Egli, Champod, and Margot, op. cit.; Neumann, C., I. Evett, and J. Skerrett. "Quantifying the Weight of Evidence from a Forensic Fingerprint Comparison: A New Paradigm.” Journal of the Royal Statistical Society, Series A, forthcoming; Neumann, Champod, Puch-Solis, et al., 2006, op. cit.; Neumann, Champod, Puch-Solis, et al., 2007, op. cit.; Perlin, M., J. Kadane, and R. Cotton. "Match Likelihood Ratio for Uncertain Genotypes." Law, Probability \& Risk, 8 (2009): 289.

${ }^{269}$ Dror, I. and J. Mnookin. "The Use of Technology in Human Expert Domains: Challenges and Risks Arising From the Use of Automated Fingerprint Identification Systems in Forensic Science.” Law, Probability \& Risk, 9 (2010): 47-67.
} 


\section{Recommendation 4.4: The federal government should continue funding research into automation of the initial quality assessment step in latent print analysis.}

\subsubsection{Latent Print Examiner Attitudes toward New Technology}

Some veteran examiners began their careers well before the advent of modern technologies such as AFIS, livescan, and digital enhancement. Younger examiners have likely worked with these technologies throughout their professional lives. Understandably, examiners vary in their comfort level in using the most sophisticated technologies. Some examiners prefer to use a magnifying glass or loupe to study hard copies of the images. Others routinely compare on-screen images.

Manufacturers should take two steps to make examiners comfortable with new technologies. First, manufacturers should ensure that new products are as usable as reasonably possible (see Chapter 7). Second, manufacturers should expand their training programs. Traditionally, training programs have focused primarily on the technical aspects of the instrumentation. Training programs also should confront the fact that some examiners may subconsciously resist new technologies that supplant the methods that they have been using for decades. In addition to teaching examiners the specific mechanics of using the new technology, the program ought to attempt to enhance the trainee's general technology skills. Chapter 8 provides more information on training improvements.

Forensic laboratory staff members should collaborate with manufacturers on the development of training programs and usable tools. As the consumers of the tools and training programs, the laboratory staff members have unique perspectives that can make a significant contribution to both development projects. Chapter 7 addresses this topic of holistic and user-centered design in more detail.

\subsection{Summary}

Technology can help latent print examiners fully analyze evidence and reach reasonable conclusions that they can defend in court. Some of these technologies, such as AFIS, livescan, and digital enhancement software, already exist; however, there is room to improve these products, and further research should be conducted to enhance these technologies.

In addition, research into probability models that allow examiners to qualify their conclusions should continue. Quantitative measures of fingerprint similarity can and have been developed. The likelihood ratio for a measurement indicates how many times more probable it is for a pair of prints from the same source to possess the measured degree of similarity than it is for a pair from different sources to be that similar. By expressing the strength of the evidence in this way and noting uncertainties in the values of the likelihood ratios, experts leave it to the judge or jury to use the degree of similarity, along with the other evidence in the case, to decide whether a pair of prints has a common source (see Chapter 6). Research into models that generate valid probabilities should continue, and examiners should receive training to explain relevant probabilities and statistics in court. Finally, research should be conducted into software to assist examiners with quantity and quality assessments for latent prints. 
Examiners and other forensic laboratory staff members should work with technology manufacturers to ensure that the products are designed for ease of use and that training programs are sufficient. Training programs should consider those examiners who may be reluctant to rely on new technology processes that augment their traditional methods of moving through the Analysis, Comparison, and Evaluation phases. Research into new and valuable technologies and the further validation of existing technologies and methods will have a positive, long-term impact on the latent print community. 
incorporated in Recommendation 3.1 (Chapter 3) and endorsed by scientific working groups from a variety of forensic disciplines, including friction ridge analysis, ${ }^{272}$ firearms and toolmarks analysis, ${ }^{273}$ and drug analysis. ${ }^{274}$ It leads to more specific recommendations that further define the minimum level of detail required for quality assurance and control purposes. Before presenting these more detailed recommendations, it is helpful to consider the role that reporting and documentation play in the legal system.

\subsection{Legal Purposes of Written Records}

\subsubsection{Pretrial Uses of Reports}

Expert reports convey useful information to the prosecution and defense before trial. "Because the reports are discoverable, they assist attorneys in preparing for trial and thus render effective representation." ${ }^{275}$ Accordingly, one federal magistrate judge recently urged that

to ensure that defense counsel can make any challenges to the admissibility of toolmark identification evidence and that courts may conduct hearings to resolve these challenges based on sufficient record, the Government should be required to strictly and timely comply with its [pretrial discovery] obligations regarding the opinions to be offered by firearms examiners in sufficient detail and sufficiently far in advance of motions deadlines or trials as to enable defense counsel to evaluate the conclusions and bases, determine whether to engage experts to test them, and if appropriate, challenge them. ${ }^{276}$

Moreover, due process principles require the prosecution to disclose information that is "favorable to the accused" 277 and "material either to guilt or to punishment," 278 as well as "evidence that the defense might have used to impeach the government's witnesses by showing bias or interest." 279 The prosecution is responsible for disclosing all such information, even if it is in the hands of the law enforcement agency. ${ }^{280}$ Making a good faith effort to ensure reports contain all known exculpatory and impeachment material helps the government comply with its disclosure obligations and avoid accusations of hiding relevant evidence. As in other fields, ${ }^{281}$ such accusations are not unheard of in regard to latent print examinations. ${ }^{282}$

${ }^{272}$ Scientific Working Group on Friction Ridge Analysis, Study and Technology. Standard for the Documentation of Analysis, Comparison, Evaluation, and Verification (ACE-V) (Latent). Version 1.0. Scientific Working Group on Friction Ridge Analysis, Study and Technology, February 2010.

${ }^{273}$ Scientific Working Group for Firearms and Toolmarks. Guidelines for the Standardization of Comparison Documentation. Scientific Working Group for Firearms and Toolmarks, October 2010.

${ }^{274}$ Scientific Working Group for the Analysis of Seized Drugs. Scientific Working Group for the Analysis of Seized Drugs (SWGDRUG) Recommendations. Revision 5.1. Scientific Working Group for the Analysis of Seized Drugs, January 2011.

${ }^{275}$ American Bar Association, op. cit., p. 73 (note omitted).

${ }^{276}$ United States v. Willock, 696 F.Supp.2d 536, 570 (D.Md. 2010).

${ }^{277}$ Brady v. Maryland, 373 U.S. 83, 87 (1963).

${ }^{278}$ Ibid.

279 Ibid.

${ }^{280}$ See Kyles v. Whitley, 514 U.S. 419, 437 (1995); Pierce v. Gilchrist, 359F.3d 1279 (10th Cir. 2004).

${ }^{281}$ Tiscareno v. Anderson, 639 F.3d 1016 (10th Cir. 2011), vacated in part, 421 Fed. Appx. 842 (10th Cir. 2011); Pierce v. Gilchrist, 359 F.3d 1279 (10th Cir. 2004). 
Finally, although the forensic expert cannot control the actions of police or prosecutors, the expert may have an ethical obligation to avoid such practices as "preparation of reports containing minimal information in order not to give the 'other side' ammunition for cross-examination," "reporting of findings without an interpretation on the assumption that if an interpretation is required it can be provided from the witness box," and "omitting some significant point from a report to trap an unsuspecting cross-examiner."283

\subsubsection{Trial Uses of Reports}

In addition to their pretrial uses, reports can assist experts who testify. Although there can be obstacles (discussed below) to introducing the report itself into evidence, documentation of the chain of custody of the evidence is crucial, and the written materials can be consulted to refresh a witness's recollection. In addition, when a report is admitted as an exhibit, it becomes part of the trial record, which allows the jury to rely on the statements in it and to consult it during jury deliberations. Admission thus can streamline the presentation and can reduce the time needed to present the results during trial. Indeed, laboratory reports sometimes are admitted by stipulation in lieu of live testimony.

When a prosecutor offers a report into evidence, however, a defendant can object that it is hearsay. Although a laboratory report offered into evidence to prove the facts recorded in it is technically hearsay, in many jurisdictions laboratory reports are admissible under an exception to the hearsay rule. ${ }^{284}$ In addition, even if the hearsay objection prevails and the report is not evidence in its own right, the expert who prepared the report or another expert may be permitted to rely on it as part of the basis for the testifying expert's own opinion. ${ }^{285}$

If the prosecutor does not present the author of the report for cross-examination at trial, a defendant also can object that the absence of the witness violates the constitutional right to confront one's accusers. The Confrontation Clause of the Sixth Amendment prevents the prosecution from introducing "testimonial" statements accusing the defendant without producing

\footnotetext{
${ }^{282}$ See, e.g., Edwards, H. "Solving the Problems that Plague the Forensic Science Community." Jurimetrics Journal of Law, Science, and Technology, 50 (2009): 9; Henson, S. "Brady Violations by DPS Fingerprint Examiners? Is Fingerprint Examination even Science?” Grits for Breakfast. Blog post, October 9, 2010. Accessed November 25 , 2011. http://gritsforbreakfast.blogspot.com/2010/10/brady-violations-by-dps-fingerprint.html.

${ }^{283}$ Lucas, D. "The Ethical Responsibilities of the Forensic Scientist: Exploring the Limits." Journal of Forensic Sciences, 34 (1989): 724. Examples of such expert advocacy by forensic serologists can be found in Garrett, B. Convicting the Innocent: Where Criminal Prosecutions Go Wrong. Harvard University Press, 2011.

${ }^{284}$ The hearsay issue is somewhat intricate. Congress expressly excluded police reports prepared for criminal prosecutions from the public records exception to the hearsay rule in Federal Rule of Evidence 803(8)(B). One line of cases reasons that this provision implies that crime laboratory reports also are outside the scope of the related Rule 803(6) business records exception to the hearsay rule. United States v. Oates, 560 F.2d 45 (2d Cir. 1977). Other federal circuits are more willing to apply the business-records exception. E.g., United States v. Baker, 855 F.2d 1353, 1359 (8th Cir. 1988). State court opinions also are conflicting. Compare Commonwealth v. Carter, 932 A.2d 1261 (Pa. 2007) (admissible hearsay), with People v. McDaniel, 670 N.W.2d 659 (Mich. 2003) (inadmissible hearsay). Some states have enacted specific rules to overcome the hearsay objection to crime laboratory reports. E.g., Massachusetts General Laws, ch. 111, §13; People v. McClanahan, 729 N.E.2d 470, 472 (Ill. 2000) (referring to Illinois statute).

${ }^{285}$ E.g., Pendergrass v. State, 913 N.E.2d 703, 709 (Ind. 2009); Kaye, D., D. Bernstein, and J. Mnookin. The New Wigmore, A Treatise on Evidence: Expert Evidence. 2nd ed. Aspen Publishers, 2011. § 4.6.
} 
the author of the statements for cross-examination. In Melendez Diaz v. Massachusetts, ${ }^{286}$ the Supreme Court applied this doctrine to sworn statements from laboratory analysts at the state Department of Public Health. Their "certificates of analysis" 287 contained "only the bare bones statement that 'the substance was found to contain: Cocaine.' At the time of trial, petitioner did not know what tests the analysts performed, whether those tests were routine, and whether interpreting their results required the exercise of judgment or the use of skills that the analysts may not have possessed." 288 The Court determined that because the "analysts' statements [were] prepared specifically for use at petitioner's trial, [they] were testimony against [the] petitioner, and the analysts were subject to confrontation under the Sixth Amendment." ${ }^{289}$ Thus, whether or not the certificates were inadmissible as hearsay, the state should not have relied on them without giving the defendant the opportunity to cross-examine the analysts themselves.

Because reports of friction ridge examinations conducted at the request of criminal investigators are also testimonial, these reports cannot serve as a complete substitute for the courtroom testimony of the examiners if the defendant objects to their introduction. Nevertheless, written records can facilitate the presentation of the prosecution's case and make the trial more efficient. Melendez Diaz noted that "it is not the case that anyone whose testimony may be relevant in establishing the chain of custody, authenticity of the sample, or accuracy of the testing device must appear in person as part of the prosecution's case." 290 Portions of a laboratory report or documentation detailing these matters might be considered non-testimonial. Moreover, the defendant's right to confrontation does not prevent the prosecution from presenting a laboratory report with the testimony of the analyst or examiner. Indeed, if the analyst is unavailable to testify, the report still may be admissible if the "defendant had a prior opportunity for cross-examination." ${ }^{291}$ In addition, there may be cases in which an expert witness who did not perform the original examination can testify to or rely on the contents of a report from the expert who did. ${ }^{292}$ Finally, like the hearsay rule, "the right to confrontation may, of course, be waived, including by failure to object to the offending evidence ...." 293 In fact, jurisdictions are free to adopt statutes or rules that enable a prosecutor to secure such a waiver by giving pretrial notice of the intent to use the report as long as "the defendant is given a period of time in which he may

\footnotetext{
${ }^{286}$ Melendez Diaz v. Massachusetts, 129 S. Ct. 2527 (2009).

${ }^{287}$ Ibid., p. 2531.

${ }^{288}$ Ibid., p. 2537.

${ }^{289}$ Ibid., p. 2540.

${ }^{290}$ Ibid., p. 2532, note 1.

${ }^{291}$ Ibid., p. 2531.

${ }^{292}$ Compare People v. Williams, 939 N.E. 2d 268, 270 (Ill. 2010), cert. granted sub nom. Williams v. Illinois, 80 U.S.L.W. 3003 (U.S. June 28, 2011) (allowing surrogate testimony from a DNA analyst at a state laboratory who compared a crime-scene DNA profile generated by a private laboratory to the profile from defendant's blood sample and determined that they matched), with Bullcoming v. New Mexico, 131 S.Ct. 2705 (2011) ("Surrogate testimony" about a defendant's blood alcohol level was inadmissible when the forensic analyst who conducted the gas chromatography, wrote a report, and signed a certificate of analysis did not testify but was not shown to be unavailable to testify and the witness who testified in his place worked at the same laboratory and was familiar with its procedures but had not participated in the testing or supervised the original analyst.)

Of course, the Confrontation Clause does not bar testimony from a fingerprint examiner who re-examines a set of images to form an independent expert opinion. The significant questions pertain only to presenting or relying on "testimonial statements" about the images made by other examiners who are not themselves made available for cross-examination. See Kaye, Bernstein, and Mnookin, op. cit., § 4.10.

${ }^{293}$ Melendez Diaz, 129 S. Ct. at 2535 n. 3.
} 
object to the admission of the evidence absent the analyst's appearance live at trial." 294 However, a more complete report could reduce the chance that the defense will demand the presence of the examiner and could enhance the chance that a laboratory supervisor or other analyst can testify at a trial if the examining analyst is unavailable.

In sum, the value of comprehensive written records in trial and pretrial proceedings reinforces Recommendation 3.1, calling for contemporaneous documentation that makes "the interpretive process as transparent as possible" and "sufficient to permit another examiner to assess the accuracy and validity of the initial examiner's assessment of the evidence." In addition, the fact that the readers of an examiner's report in a case scheduled for trial are likely to be lawyers, judges, or jurors prompts a related recommendation directed specifically at the examiner's summary report:

\section{Recommendation 5.1: The report of the examination should ensure that the findings and their limitations are intelligible to non-experts.}

This recommendation finds support in the views of legal and scientific organizations, such as the American Bar Association ${ }^{295}$ and the National Research Council, ${ }^{296}$ as well as forensic science working groups and organizations, such as the European Network of Forensic Science Institutes $^{297}$ and the Scientific Working Group for the Analysis of Seized Drugs. ${ }^{298}$ The European Network, for example, explains that

the reports of forensic investigations will be read by a wide variety of individuals, many of whom will have little knowledge of science. They may have to be read aloud in courts of law to inform judges and advocates and, in some jurisdictions, members of a jury. Reports must, therefore, be written as clearly and unambiguously as possible. Great care must be taken to ensure that all details relating to the examinations undertaken, and the scientific rationale on which the examinations are based, are described in language that can be understood by nonscientists. ${ }^{299}$

\footnotetext{
${ }^{294}$ Ibid., p. 2541. If the defendant does object, it appears that the state cannot place the burden of calling the witness on the defendant. Briscoe v. Virginia, 130 S.Ct. 1316 (2010).

${ }^{295}$ American Bar Association, op. cit., Standard 16-3.3(c).

${ }^{296}$ National Academy of Sciences, National Research Council, Committee on Identifying the Needs of the Forensic Science Community. Strengthening Forensic Science in the United States: A Path Forward. National Academies Press, 2009, p. 186; National Academy of Sciences, National Research Council Committee on Scientific Assessment of Bullet Lead Elemental Composition Comparison. Forensic Analysis: Weighing Bullet Lead Evidence. National Academies Press, 2004, p. 110.

${ }^{297}$ European Network of Forensic Science Institutes Standing Committee for Quality and Competence. Performance Based Standards for Forensic Science Practitioners. European Network of Forensic Science Institutes Standing Committee for Quality and Competence, July 2004.

${ }^{298}$ Scientific Working Group for the Analysis of Seized Drugs. Supplemental Document SD-1 for Part I, A Code of Professional Practice for Drug Analysts. Recommendation 2.3.1. Scientific Working Group for the Analysis of Seized Drugs, October 2004.

${ }^{299}$ European Network, op. cit., Activity I.
} 


\subsection{Minimal Contents of a Report}

There is no hard and fast line between the information that should be recorded in reports versus contemporaneous, supporting documentation. One laboratory could include considerable detail in a report prepared for police or litigants, while another might store much of the same documentation elsewhere and write a shorter report that simply refers to the underlying documentation. Therefore, the Working Group does not propose a one-size-fits-all division of the necessary information. Instead, the outline below contains the minimum content of a report and indicates what additional information should be recorded, either in the report or in related documentation. This chapter's appendices offer sample reports of varying length and detail.

Broadly speaking, a report should describe the items submitted to the latent print examiner, how the examiner processed these items, and how the examiner reached any conclusions. Although it has been said that "case documentation is not complete if the record-keeping process does not begin at the crime scene, ${ }^{\prime 300}$ and a full report certainly could describe the entire chain of evidence, the focus here is on the processing, examination, and conclusions about the material after it is delivered to a latent print examiner. The essential contents of a report on these phases of evidence assessment are listed at the conclusion of this section in Recommendation 5.2, and the appendices to this chapter present sample reports. The recommendation covers such matters as reporting on each step of the Analysis, Comparison, Evaluation, and Verification (ACE-V) process; noting non-essential, domain-irrelevant information known to the examiner (if any); noting the existence of documentation outside the report itself; and presenting results and defining terms.

\subsubsection{Images of Friction Ridge Impressions}

Friction ridge impressions on surfaces may be examined on that surface, developed from that surface, or placed into another format (such as a photograph or scan) for analysis and comparison. When a development process was used, or when the image was copied into another format, the report should define the process that was used and indicate that an image of the developed friction ridge impression was retained.

\subsubsection{List of Comparisons}

Latent prints can be compared to other latent prints to see if the same individual was present at both locations. They can be compared to a ten-print card or other exemplar from a known individual. In addition, exemplars from an individual whose identity is in question can be compared to exemplars from known individuals. The report should list which images were compared to one another.

Traditionally, latent prints have been compared with ten-print cards for pre-existing suspects in a case. The creation of large databases of computer-searchable images permits comparisons to individuals culled with automated fingerprint identification system (AFIS) software (Chapter 4).

\footnotetext{
${ }^{300}$ Peterson, P., C. Dreyfus, M. Gische, et al. "Latent Prints: A Perspective on the State of the Science." Forensic Science Communications, 11, no. 4 (October 2009).
} 
If an AFIS search has been conducted, the report should specify the results. A record of the AFIS candidate list should be retrievable.

\subsubsection{Method and Conclusions}

Just as a report should indicate how latent prints were made visible, it also should state the procedure used to make comparisons between friction skin impressions and to draw conclusions from these comparisons. ${ }^{301}$ The conventional procedure for associating latent and known prints involves the ACE-V process described in Chapter 1. Traditionally, the outcome of ACE-V has been expressed in terms of three possible categories: individualization, exclusion, or inconclusive. ${ }^{302}$ Nonetheless, scientific research or reasoning does not limit reported results to these three categories. ${ }^{303}$ A more finely graded set of categories is possible (see, for example, Chapter 9), and the computer-based methods described in the preceding chapter are another way to measure the features of prints and the degree of similarity between pairs of prints. As automated systems advance and are further validated with large data sets, they will be useful in conjunction with (or conceivably, at some time in the future, instead of) ACE-V. But, whatever procedures are employed to draw inferences from the evidence-ACE-V or otherwise - the report should designate them and should frame the conclusions in a manner that is accurate and scientifically appropriate.

\subsubsection{Limitations}

For the sake of lay readers, a report may contain some general warnings that indicate its limits. For example, if no comparisons were made, the report might note that the absence of any usable latent prints, or even any smudges, does not mean that the surface was never touched. If the examiner associates the latent print with a known finger, the report might observe that, standing alone, this association does not indicate how, why, or when the latent print was deposited. Statements like these are included in reports of the Federal Bureau of Investigation (FBI). They are not necessary to present the results of an examination or to document how it was conducted, but they may be helpful for some readers.

In addition, if there are significant reasons to question any conclusions, these should be noted. ${ }^{304}$ As the Scientific Working Group for the Analysis of Seized Drugs concluded, forensic analysts have the professional responsibility to present both written and oral "advice ... in a clear and objective manner" 305 — a duty that entails "considering and providing alternative explanations or

\footnotetext{
${ }^{301}$ Compare American Society of Crime Laboratory Directors. Guidelines for Forensic Laboratory Management Practices. American Society of Crime Laboratory Directors, 1987.

${ }^{302}$ Scientific Working Group on Friction Ridge Analysis, Study and Technology. Standards for Conclusions. Version 1.0. Scientific Working Group on Friction Ridge Analysis, Study and Technology, September 2003.

${ }^{303}$ Budowle, B., J. Buscaglia, and R. Perlman. "Review of the Scientific Basis for Friction Ridge Comparisons as a Means of Identification: Committee Findings and Recommendations." Forensic Science Communications, 8 (January 2006); Haber, L. and R. Haber. Challenges to Fingerprints: A Guidebook for Prosecution and Defense and Examiners. Lawyers and Judges Publishing Co., 2009.

${ }^{304}$ European Network, op. cit., Standard I1(c); NAS, NRC, Committee on Identifying the Needs of the Forensic Science Community, op. cit., pp. 21-22; SWGDRUG, 2004, op. cit., Recommendation 2.3.1. But see Scientific Working Group on Materials Analysis. Forensic Human Hair Examination Guidelines. Scientific Working Group on Materials Analysis, April 2005.

${ }^{305}$ SWGDRUG, 2011, op.cit., Recommendation 2.3(a).
} 
interpretations for their findings, where appropriate; making clear the strengths and any limitations in their advice or evidence; [and] declaring anything that might undermine the integrity of their evidence or its use ...." ${ }^{, 306}$

\subsubsection{Verification and Consultation}

If the examiner followed the ACE-V process, the report should disclose or refer the reader to the record of who verified the results, when verification occurred, and what information the verifying examiner had about the previous comparisons. If the Verification phase involved a difference of opinion, then the existence of the disagreement should be noted in the report and the basis for the final consensus should be stated in the report or other documentation. ${ }^{307}$

In some cases, an examiner seeks guidance from another expert (examiner or supervisor) before reaching a conclusion. Consultation is a way of sharing expertise and does not mean that there is a conflict. A poor quality unknown print, a poor quality known print, an irregular substrate, excessive deposition or lateral pressure, limited Level 2 Detail (see Chapters 3), and other factors could lead an examiner to consult with others. A conflict occurs when an examiner does not agree with another examiner during the verification, technical review, or administrative review. Such a disagreement is resolved in discussions among examiners who initially reached different conclusions or with the involvement of supervisory personnel up to the level of the laboratory director, if necessary. The recommendation for disclosure applies to conflicts rather than consultations.

\subsubsection{Context}

Chapters 1 and 3 discuss the potentially biasing effects of contextual information. As those chapters explained, observers' expectations have been shown to influence judgment in a broad range of tasks. Because "the possibility of biases influencing the decision making process of examiners" cannot be dismissed, ${ }^{308}$ a report should reveal the context of the examination by describing or referring the reader to the information about the case that an examiner received. For example, the possibility of an expectancy effect in comparing and evaluating a pair of prints argues in favor of revealing whether an examiner was informed that the exemplars were for elimination purposes, that they came from suspects in the case, or that they were the result of an AFIS search. ${ }^{309}$

Chapter 3 observes that in some laboratories, examiners are exposed to a great deal of information that is clearly not related to or necessary for their analysis and calls upon laboratories to minimize the amount of extraneous, domain-irrelevant information that is

${ }^{306}$ SWGDRUG, 2004, op. cit., Recommendation 2.3.1.

${ }^{307}$ Compare SWGFAST, 2010, op. cit., Standard 5.

${ }^{308}$ SWGFAST, 2009, op. cit.

${ }^{309}$ Dror, I. and J. Mnookin. "The Use of Technology in Human Expert Domains: Challenges and Risks Arising From the Use of Automated Fingerprint Identification Systems in Forensic Science." Law, Probability \& Risk, 9 (2010): 47 - 67. An additional reason to document the fact of an AFIS search arises when candidates from this search are excluded. These exclusions increase the probative value of a match to a suspect. Aitken, C. and F. Taroni. Statistics and the Evaluation of Evidence for Forensic Scientists. 2nd ed. John Wiley \& Sons, 2004; Kaye, D. "Rounding Up the Usual Suspects: A Legal and Logical Analysis of DNA Database Trawls." North Carolina Law Review, 87, no. 2 (2009): 425 -503. For a modest number of exclusions, however, the effect is small. 
provided to examiners. The recommendation here complements this suggestion by asking for disclosure of the information actually provided to examiners. This does not mean that the examiner must write out everything that is known about the background of the case. For example, if an examiner was exposed to a police case file or report containing information about the suspect's criminal record or the suspect's admissions to the police, the report could simply note that the examiner read the police report that contained information about the suspect before reaching a conclusion.

\subsubsection{References to Other Documentation}

The report itself should at least describe the highlights of the examination of the evidence, and it should be an "accurate summary of significant material contained in the case notes." 310 But it need not present every detail of the process. If documentation, such as bench notes or additional images, is not appended to the report, the report should note that these materials are on file and can be obtained from the laboratory upon request. ${ }^{311}$

For example, if more than one examiner reached a conclusion about the sufficiency or similarity of two images, the conclusions should be recorded in the documentation and noted in the summary report. The quality assurance and quality control measures in place also should be noted. Because these measures would be spelled out in written protocols and statements of standard operating procedures, it should be sufficient to refer to these documents.

The Scientific Working Group on Friction Ridge Analysis, Study and Technology (SWGFAST) suggests that "the substrate, development medium, or preservation method can have a significant impact on the appearance of a latent print" and that "additional analytical factors, particularly on complex prints, provide the basis for distortion interpretation and explanations for variation in appearance." 312 Consequently, the documentation should record information about the substrate, development medium, and preservation method (e.g., lift, photograph, or legible copy).

The results of the effort to develop prints also should be documented by annotated images or written descriptions that record how many and which prints were determined to be suitable for comparison as well as the existence of prints determined to be unsuitable for comparison. ${ }^{313}$ If the latent print examiner uses enhancement technology, he or she should retain the unenhanced image and maintain an audit trail of all digital enhancements to subsequent copies (see Chapter 4). The FBI laboratory suggests that

once the evidence has been received in the Latent Print Unit for processing, case note documentation should chronologically include each activity and the results of the activity. Documentation should include any development techniques applied, the date the process was applied, and the result. The documented result should include the presence or absence of any prints and the indication of whether the developed prints are suitable for capture either through scanning or photography.

\footnotetext{
${ }^{310}$ ASCLD, op. cit., p. 43.

${ }^{311}$ See European Network, op. cit., Standard I1(f).

${ }^{312}$ SWGFAST, 2010, op. cit., p. 2.

${ }^{313}$ Compare ibid., Standard 1.1.2.
} 
This documentation process should continue until all processing techniques have been applied or the processing is discontinued. ${ }^{314}$

Furthermore, it recommends that

case documentation should indicate the number of latent prints of value detected on each item, along with a description of the item, and which items did not contain any latent prints or latent prints of value. Additional requirements include the disposition of any images containing latent prints of value and any images of latent prints that were not analyzed, compared, or evaluated. ${ }^{315}$

SWGFAST suggests that "documentation ... may be accomplished by making a 'no value' notation (e.g., 'NV') on a lift, photograph, or legible copy retained as part of the case record [or] by indicating in case notes that "no value' impressions are present on a lift or photograph." 316 Although SWGFAST adds that "copies of latent prints that are of no value do not need to be retained in the case record," this Working Group recommends retaining all images for possible later review.

Finally, the report or associated documentation should record the features that the examiner(s) considered and relied on in reaching a conclusion of identification, including those features used in any verification. All comparisons conducted during the course of the examination, including latent to exemplar, latent to latent, and exemplar to exemplar, should be documented. In some cases, a number of exclusions may be made. For example, an AFIS search will generate a list of candidates. When, in screening these candidates, exemplars are readily excluded, the documentation need not show the specific feature or features used for the exclusion, but the exemplars should be retained or be kept retrievable. ${ }^{317}$

The report or supporting documentation should explain, through annotations on images or in some other fashion, the features of the unknown that were selected for analysis and used for comparison. ${ }^{318}$ An apparent dissimilarity that is attributed to distortion or another cause should be noted. ${ }^{319}$ Providing this information complies with the International Organization for Standardization's ISO/IEC 17025: 2005, clause 5.10.5, ${ }^{320}$ which requires that "the laboratory shall document the basis upon which the opinions and interpretations have been made" and responds to the concern that examiners might fail "to document which features within a latent print support their reasoning and conclusions." 321 A verifying examiner's notes or other materials should document the verification in similar detail.

As discussed in Chapter 3, contemporaneous documentation of each major step in the examination process can help ensure the examination is conducted properly and thus reduce the

\footnotetext{
314 Peterson, Dreyfus, Gische, et al., op. cit.

${ }^{315}$ Ibid.

${ }^{316}$ SWGFAST, 2010, op. cit., p. 3.

${ }^{317}$ Ibid., Standard 2.4.

${ }^{318}$ Ibid., Preamble. See also Chapter 3.

${ }^{319}$ Compare SWGFAST, 2010, op. cit., p. 2.

${ }^{320}$ International Organization for Standardization. General Requirements for the Competence of Testing and Calibration Laboratories, ISO/IEC 17025:2005(E), 2d ed. International Organization for Standardization, 2005.

${ }^{321}$ NAS, NRC, Committee on Identifying the Needs of the Forensic Science Community, op. cit., p. 143.
} 
chance of error. It also can provide a record that might be useful for testimony by the examiner or other possible expert witnesses at a later time.

\subsubsection{Definition of Terms}

A report should define important technical terms, either explicitly or by reference to an authoritative, readily available source. For example, latent print examiners often refer to "individualization" as "identification," 322 but in ordinary parlance, an identification need not be an assertion that only one person on Earth could be the source of an impression. An eyewitness description might be used to "identify" a suspect, even though the individual so identified would not be the only person in the world to fit the description. Even the word "individualize" is not self-defining. It might mean that in a closed group of, say, seven suspects, only one suspect's finger could be the source of a latent print, or it might represent a more powerful assertion that no other finger that has ever been or ever will be in existence can produce so similar a latent print. An explicit definition informs the reader of the intended meaning, and Chapter 1 discusses ways to define terms relating to the process of establishing an association between a known individual and a latent print. The importance of defining terms clearly is widely recognized in the forensic sciences, ${ }^{323}$ and this part of the recommendation also follows from Recommendation 5.1 on making the report understandable to lay readers.

Recommendation 5.2: A report should:

a. Identify the latent print examiner(s);

b. Describe the items submitted to the examiner(s);

c. List the procedures used by the examiner to develop, visualize, or enhance the friction ridge impressions;

d. List all comparisons conducted;

e. State all conclusions with the method used to reach them;

f. Note any important limitations to the conclusions;

g. Indicate whether a verification was made and whether there was any conflict of opinion among examiners prior to the reported conclusions;

h. Note (or refer to external documentation of) any information about the case that the examiner(s) received;

i. Note the existence of additional documentation; and

j. Define important technical terms, either explicitly or by reference to an authoritative, readily available source.

\footnotetext{
322 E.g., SWGFAST, 2003, op. cit., Standard 1. A draft of the document intended to replace these standards does not use the word "identification." Scientific Working Group on Friction Ridge Analysis, Study and Technology. Draft for Comment: Standards for Examining Friction Ridge Impressions and Resulting Conclusions (To Replace: Friction Ridge Examination Methodology for Latent Print Examiners, Version 1.01, and Standards for Conclusions, Version 1.0). Version 1.0. Scientific Working Group on Friction Ridge Analysis, Study and Technology, August 2010, p. 4. Another draft document, the Scientific Working Group on Friction Ridge Analysis, Study and Technology. Standard Terminology of Friction Ridge Examination (To Replace: Glossary, Version 2.0). Version 3. Scientific Working Group on Friction Ridge Analysis, Study and Technology, February 11, 2011, lists "individualization" as the primary meaning of "identification."

${ }^{323}$ For example, European Network, op. cit., Activity I. SWGDRUG, 2004, op. cit., Recommendation 3.2.1.
} 


\subsection{Summary}

A report and documentation that contain the information listed in these recommendations provide an accessible record of the work of latent print examiners. The level of detail is sufficient to inform investigators, prosecutors, defense counsel, other experts, and judges or juries as to how the analysis was conducted and what conclusions were reached. This type of reporting meets scientific norms, quality assurance, and legal concerns. Appendices offer sample language for a full report, a shortened report with minimal requirements, and a report with qualified conclusions. 


\section{Appendix 5-A: Sample Report Summary}

Bureau of Investigation

Laboratory Division, Latent Print Section

123 Main Street

Washington, D.C. 20035

\section{SAMPLE REPORT OF EXAMINATION}

To: $\quad$ BI Agent John Dunne

Date: October 20, 2011

Los Angeles Field Office

Case Number: LA-123456

Case Title: Bank of Los Angeles

123 California Blvd.

Los Angeles, CA

Aug. 28, 2011

Bank Robbery

Date specimens received: September 3, 2011

Fingerprint Analyst: Susanne Brown

The items listed below were examined in the Latent Print Section.

\begin{tabular}{|c|l|}
\hline Item \# & \multicolumn{1}{|c|}{ Description } \\
\hline 1 & Demand note beginning, "I have a gun..." \\
\hline 2 & Bank of Los Angeles withdrawal form \\
\hline 3 & Pen with chain \\
\hline 4 & $\begin{array}{l}\text { Lift indicated as coming from customer } \\
\text { counter }\end{array}$ \\
\hline
\end{tabular}

Table 1: Items examined in Latent Print Section

\section{Results of Examinations}

Items of evidence submitted to the Latent Print Section for examination may be examined visually, examined with various light sources, or processed with chemicals and powders to detect the presence of latent friction ridge prints. The specific sequence of examinations and processes depends upon the nature of the evidence. ${ }^{1}$ I applied the following processes to the submitted items as follows:

\footnotetext{
${ }^{1}$ See Bureau of Investigation, Processing Guide for Developing Latent Prints, Revised 2001.
} 


\begin{tabular}{|c|l|l|}
\hline $\begin{array}{c}\text { Item } \\
\#\end{array}$ & \multicolumn{1}{|c|}{ Description } & \multicolumn{1}{|c|}{ Processing techniques } \\
\hline 1 & Demand note beginning, "I have a gun..." & $\begin{array}{l}\text { Visual, LASER, ultraviolet, } \\
\text { Crimescope, 1,8-Diazafluoren-9- } \\
\text { one/LASER, Ninhydrin, Physical } \\
\text { Developer }\end{array}$ \\
\hline 2 & Bank of Los Angeles withdrawal form & $\begin{array}{l}\text { Visual, LASER, ultraviolet, } \\
\text { Crimescope, 1,8-Diazafluoren-9- } \\
\text { one/LASER, Ninhydrin, Physical } \\
\text { Developer }\end{array}$ \\
\hline 3 & Pen with chain & $\begin{array}{l}\text { Visual, LASER, ultraviolet, } \\
\text { Crimescope, Cyanoacrylate Fuming, } \\
\text { reflective ultraviolet imaging system, } \\
\text { Cyanoacrylate Dye } \\
\text { Stain/LASER/ultraviolet/Crimescope, } \\
\text { White Powder }\end{array}$ \\
\hline 4 & $\begin{array}{l}\text { Lift indicated as coming from customer } \\
\text { counter }\end{array}$ & $\begin{array}{l}\text { Visual-see crime scene log for } \\
\text { additional information }\end{array}$ \\
\hline
\end{tabular}

Table 2: Processing techniques applied to submitted items

BI conducts friction ridge print examinations using the Analysis, Comparison, Evaluation, and Verification (ACE-V) process. The first phase in the process is Analysis, which is conducted independently on first the latent then the known prints. During this phase, each print is analyzed for both the quality and quantity of information present. The qualitative assessment is based upon the clarity of the friction ridges, the substrate on which the latent print was developed, deposition and lateral pressures, and the processing techniques applied. The quantitative analysis takes into account all of the information present in the print, broken down into three levels of detail. Level 1 Detail consists of the overall ridge flow, including pattern type and orientation of the print based upon that ridge flow. Level 2 Detail lies in individual ridge paths, including those of continuous ridges as well as ridges that come to an end, divide to form two or more ridges, or appear as isolated dots. Analysis of these ridge characteristics accounts for their type, direction, location, and spatial sequence. Level 3 Detail includes individual ridge attributes, particularly shape and width along the length of each ridge. The quality and quantity of information observed during the Analysis phase determines whether the print contains suitable information to conduct a comparison with another print.

\section{Results of Analysis}

I detected four latent fingerprints on Items 1, 2, and 4 that were suitable for comparison: 


\begin{tabular}{|c|l|c|c|}
\hline $\begin{array}{c}\text { Item } \\
\#\end{array}$ & \multicolumn{1}{|c|}{ Description } & $\begin{array}{c}\text { \# of } \\
\text { prints }\end{array}$ & Processing technique \\
\hline 1 & Demand note beginning, "I have a gun..." & 2 & $\begin{array}{c}1 \text { 1,8-Diazafluoren-9- } \\
\text { one/LASER, 1 Ninhydrin }\end{array}$ \\
\hline 2 & Bank of Los Angeles withdrawal form & 1 & Ninhydrin \\
\hline 4 & $\begin{array}{l}\text { Lift indicated as coming from customer } \\
\text { counter }\end{array}$ & 1 & Black powder lift \\
\hline
\end{tabular}

Table 3: Prints determined to be suitable for comparison and the processing technique used to detect them

In the Comparison phase of the ACE-V process, I conducted a side-by-side comparison of a latent print with an exemplar. I examined both prints for similarities and differences, assessing ridges sequentially for agreement or disagreement in all levels of detail.

In the Evaluation phase of the ACE-V, I considered all of the information gathered during Analysis and Comparison to reach conclusions about the origin of the latent prints. I made identifications as well as exclusions. An exclusion is an opinion that the two prints did not originate from the same source because there is information in disagreement in the comparable areas of the two prints that would not be present if the prints came from the same finger. An identification is an opinion that the two prints originated from the same source because the information in the comparable areas of two prints is in sufficient agreement. An identification does not necessarily eliminate the possibility that another person in the world could leave a print with areas of similar agreement. It means that within the examiner's experience and knowledge, no other prints with this much similarity have come from different people.

\section{Results of Comparison and Evaluation}

After a conversation with the case agent (see Table 7), I compared the four latent fingerprints to the fingerprints of THOMAS SMITH, BI \#123456, with the following results:

\begin{tabular}{|c|l|c|c|}
\hline $\begin{array}{c}\text { Item } \\
\#\end{array}$ & \multicolumn{1}{|c|}{ Description } & $\begin{array}{c}\text { \# of } \\
\text { prints }\end{array}$ & $\begin{array}{c}\text { Results of comparison with } \\
\text { THOMAS SMITH, BI } \\
\# \mathbf{1 2 3 4 5 6}\end{array}$ \\
\hline 1 & Demand note beginning, "I have a gun..." & 2 & 2 Identifications \\
\hline 2 & Bank of Los Angeles withdrawal form & 1 & Exclusion \\
\hline 4 & $\begin{array}{l}\text { Lift indicated as coming from customer } \\
\text { counter }\end{array}$ & 1 & Exclusion \\
\hline
\end{tabular}

Table 4: Results of comparisons with THOMAS SMITH, BI \#123456

The remaining two unidentified latent fingerprints were searched in the BI automated fingerprint identification system (AFIS). I reviewed the resulting list of candidates (available upon request to the $\mathrm{BI}$ ) and reached the following conclusions: 


\begin{tabular}{|c|l|c|c|}
\hline $\begin{array}{c}\text { Item } \\
\#\end{array}$ & \multicolumn{1}{|c|}{ Description } & $\begin{array}{c}\text { \# of } \\
\text { prints }\end{array}$ & Results of AFIS search \\
\hline 2 & Bank of Los Angeles withdrawal form & 1 & No Identification effected \\
\hline 4 & $\begin{array}{l}\text { Lift indicated as coming from customer } \\
\text { counter }\end{array}$ & 1 & $\begin{array}{c}\text { Identification with JANE } \\
\text { JONES, BI \#987654 }\end{array}$ \\
\hline
\end{tabular}

Table 5: Results of AFIS searches

The remaining unidentified latent fingerprint (on item \#2) is not a fingerprint of JANE JONES, BI \#987654.

\section{Summary of Evaluation}

\begin{tabular}{|c|l|c|c|}
\hline $\begin{array}{c}\text { Item } \\
\#\end{array}$ & \multicolumn{1}{|c|}{ Description } & $\begin{array}{c}\text { \# of } \\
\text { prints }\end{array}$ & Evaluation Summary \\
\hline 1 & Demand note beginning, "I have a gun..." & 2 & $\begin{array}{c}\text { 2 Identifications with } \\
\text { THOMAS SMITH, BI } \\
\# 123456\end{array}$ \\
\hline 2 & Bank of Los Angeles withdrawal form & 1 & $\begin{array}{c}\text { Not a fingerprint of } \\
\text { THOMAS SMITH, BI } \\
\# 123456, \text { or JANE JONES, } \\
\text { BI \#987654 }\end{array}$ \\
\hline 4 & $\begin{array}{l}\text { Lift indicated as coming from customer } \\
\text { counter }\end{array}$ & 1 & $\begin{array}{c}\text { Identification with JANE } \\
\text { JONES, BI \#987654 }\end{array}$ \\
\hline
\end{tabular}

Table 6: Summary of evaluation

The presence of a friction ridge print on an item of evidence indicates contact was made between the source and the item of evidence. The presence of a friction ridge print alone does not necessarily indicate the significance of either the contact or the time frame during which the contact occurred.

Due to the many factors involved in the deposition of a friction ridge print, neither the absence of a friction ridge print on evidence nor the exclusion of a friction ridge print with a given source necessarily precludes that source from having touched the evidence.

\section{Results of Verifications and Blind Verifications}

The Verification phase of the ACE-V process consists of the application of the Analysis, Comparison, and Evaluation phases of the ACE-V process to a friction ridge print by another qualified examiner who then expresses his or her agreement or disagreement with the original examiner's conclusion. On October 18, 2011, Fingerprint Analyst Robert Johnson, knowing the outcome of the original examination, verified the identifications. There were no conflicts of opinion.

On October 19, 2011, Fingerprint Analyst James Bishop conducted a blind verification of the identification with JANE JONES. (In blind verifications, the verifying examiner is unaware of the original examiner's conclusion.) A conflict of opinion occurred during this blind verification 
of the identification with JANE JONES. Initially, the verifier deemed the comparison inconclusive due to poor quality exemplars. Upon consultation with the primary analyst, the blind verifier realized that he had not received all available exemplars. After comparing the additional exemplars, the verifier concurred with the identification decision.

Additional documentation, including bench notes and annotated images of the latent prints for both the primary analyst and verifiers, is retained as part of the case record and can be provided upon request. The BI Laboratory Division's Quality Assurance Manual and Standard Operating Procedures can be found online at www.BI.org.

\section{Case Information Received by the Fingerprint Analyst}

The following table summarizes the case-related information that I received for this case:

\begin{tabular}{|l|l|l|}
\hline \multicolumn{1}{|c|}{ Date } & \multicolumn{1}{|c|}{$\begin{array}{c}\text { Communication } \\
\text { Type }\end{array}$} & \multicolumn{1}{c|}{ Description } \\
\hline $\begin{array}{l}\text { September 3, } \\
2011\end{array}$ & Incoming letter & $\begin{array}{l}\text { Letter from contributor submitted with the evidence. } \\
\text { Details of case, including type of crime, location } \\
\text { of crime, date of crime, description of how bank } \\
\text { robbery occurred, and description of evidence being } \\
\text { submitted were included in the letter. No individuals } \\
\text { to compare were provided. }\end{array}$ \\
\hline $\begin{array}{l}\text { September 14, } \\
2011\end{array}$ & $\begin{array}{l}\text { Telephone call } \\
\text { (documented } \\
\text { on Activity \& } \\
\text { Communication Log) }\end{array}$ & $\begin{array}{l}\text { Telephone call with Case Agent naming individual } \\
\text { to compare. THOMAS SMITH, BI \#123456, had } \\
\text { been developed as a suspect due to information from } \\
\text { a confidential informant. }\end{array}$ \\
\hline
\end{tabular}

Table 7: Information on case received by examiner

For questions about the content of this report, please contact Fingerprint Analyst Susanne Brown at (202) 123-4567.

The specimens are being returned under separate cover.

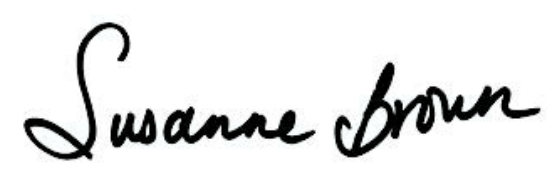

Susanne Brown

Fingerprint Analyst 


\section{Appendix 5-B: Sample Report Summary, Short Version}

Bureau of Investigation

Laboratory Division, Latent Print Section
123 Main Street

Washington, D.C. 20035

\section{SAMPLE REPORT OF EXAMINATION}

To: $\quad$ BI Agent John Dunne

Date: October 20, 2011

Los Angeles Field Office

Case Number: LA-123456

Case Title: Bank of Los Angeles

123 California Blvd.

Los Angeles, CA

Aug. 28, 2011

Bank Robbery

Date specimens received: September 3, 2011

Fingerprint Analyst: Susanne Brown

The items listed below were examined in the Latent Print Section.

\begin{tabular}{|c|l|}
\hline Item \# & \multicolumn{1}{|c|}{ Description } \\
\hline 1 & Demand note beginning, "I have a gun..." \\
\hline 2 & Bank of Los Angeles withdrawal form \\
\hline 3 & Pen with chain \\
\hline 4 & $\begin{array}{l}\text { Lift indicated as coming from customer } \\
\text { counter }\end{array}$ \\
\hline
\end{tabular}

Table 1: Items examined in Latent Print Section

Items of evidence submitted to the Latent Print Section for examination may be examined visually, examined with various light sources, or processed with chemicals and powders to detect the presence of latent friction ridge prints. The specific sequence of examinations and processes depends upon the nature of the evidence. ${ }^{1}$ A complete list of the processes used in this submission is maintained in the case record and can be furnished upon request.

\footnotetext{
${ }^{1}$ See Bureau of Investigation, Processing Guide for Developing Latent Prints, Revised 2001.
} 


\section{Results of Examinations}

Four latent fingerprints were detected on the items and compared to THOMAS SMITH, BI \#123456, with the following results:

\begin{tabular}{|c|l|c|c|}
\hline $\begin{array}{c}\text { Item } \\
\#\end{array}$ & \multicolumn{1}{|c|}{ Description } & $\begin{array}{c}\text { \# of } \\
\text { prints }\end{array}$ & $\begin{array}{c}\text { Results of comparison with } \\
\text { THOMAS SMITH, BI } \\
\# \mathbf{1 2 3 4 5 6}\end{array}$ \\
\hline 1 & Demand note beginning, "I have a gun..." & 2 & 2 Identifications \\
\hline 2 & Bank of Los Angeles withdrawal form & 1 & 1 Exclusion \\
\hline 3 & Pen with chain & 0 & N/A \\
\hline 4 & $\begin{array}{l}\text { Lift indicated as coming from customer } \\
\text { counter }\end{array}$ & 1 & 1 Exclusion \\
\hline
\end{tabular}

Table 2: Results of comparisons with THOMAS SMITH, BI \#123456

The remaining unidentified latent fingerprints were searched in the BI automated fingerprint identification system (AFIS) with the following results:

\begin{tabular}{|c|l|c|c|}
\hline $\begin{array}{c}\text { Item } \\
\#\end{array}$ & \multicolumn{1}{|c|}{ Description } & $\begin{array}{c}\text { \# of } \\
\text { prints }\end{array}$ & Results of AFIS search \\
\hline 2 & Bank of Los Angeles withdrawal form & 1 & No Identification effected \\
\hline 4 & $\begin{array}{l}\text { Lift indicated as coming from customer } \\
\text { counter }\end{array}$ & 1 & $\begin{array}{c}\text { Identification with JANE } \\
\text { JONES, BI \#987654 }\end{array}$ \\
\hline
\end{tabular}

Table 3: Results of AFIS searches

The remaining unidentified latent fingerprint (on item \#2) is not a fingerprint of JANE JONES, BI \#987654.

Friction ridge print examinations are conducted using the Analysis, Comparison, Evaluation, and Verification (ACE-V) process. The steps of ACE-V are applied to each examination as appropriate. Analysis is the assessment of the quantity and quality of the information present in the print to determine if sufficient reliable details are present to conduct a comparison with another print. Comparison is the side-by-side observation of friction ridge prints to determine whether or not the information in two prints is in agreement. Evaluation is the formulation of a conclusion based upon the information gathered during analysis and comparison. The evaluation can result in an exclusion, an identification, or an inconclusive outcome. An exclusion is an opinion that the two prints did not originate from the same source because there is information in disagreement in the comparable areas of two prints that would not be present if the prints came from the same finger. An identification is an opinion that the two prints originated from the same source because the information in the comparable areas of two prints is in sufficient agreement. An identification does not necessarily eliminate the possibility another person in the world could leave a print with areas of similar agreement. It means that within the examiner's experience and knowledge, no other prints with this much similarity have come from different people. Verification is the independent application of the Analysis, Comparison, and Evaluation phases of the ACE-V process to a friction ridge print by another examiner. 
The presence of a friction ridge print on an item of evidence indicates that contact was made between the source and the item of evidence. The presence of a friction ridge print alone does not necessarily indicate the significance of the contact or the time frame during which the contact occurred.

Due to the many factors involved in the deposition of a friction ridge print, neither the absence of a friction ridge print on evidence nor the exclusion of a friction ridge print with a given source necessarily precludes that source from having touched the evidence.

Verifications of the identifications were conducted by Fingerprint Analyst Robert Johnson, knowing the outcome of the original examination, on October 18, 2011. There were no conflicts of opinion.

On October 19, 2011, Fingerprint Analyst James Bishop conducted a blind verification of the identification with JANE JONES. (In blind verifications, the verifying examiner is unaware of the original examiner's conclusion.) A conflict of opinion occurred during this blind verification of the identification with JANE JONES. Initially, the verifier deemed the comparison inconclusive due to poor quality exemplars. Upon consultation with the primary analyst, the blind verifier realized that he had not received all available exemplars. After comparing the additional exemplars, the verifier concurred with the identification decision.

Additional documentation, including bench notes, annotated images of the latent prints for both the primary analyst and verifier, and communications indicating the case information received by the analyst and the date on which that information was received are retained as part of the case record and can be provided upon request. The BI Laboratory Division's Quality Assurance Manual and Standard Operating Procedures can be found online at www.BI.org.

For questions about the content of this report, please contact Fingerprint Analyst Susanne Brown at (202) 123-4567.

The specimens are being returned under separate cover.

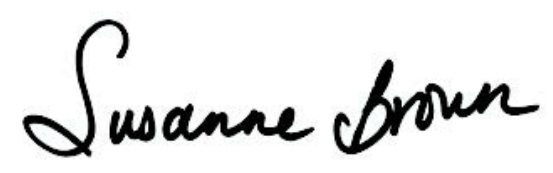

Susanne Brown Fingerprint Analyst 


\section{Appendix 5-C: Sample Report Summary with Qualified Conclusions}

Bureau of Investigation

Laboratory Division, Latent Print Section
123 Main Street

Washington, D.C. 20035

\section{SAMPLE REPORT OF EXAMINATION}

To: $\quad$ BI Agent John Dunne

Date: October 20, 2011

Los Angeles Field Office

Case Number: LA-123456

Case Title: Bank of Los Angeles

123 California Blvd.

Los Angeles, CA

Aug. 28, 2011

Bank Robbery

Date specimens received: September 3, 2011

Fingerprint Analyst: Susanne Brown

The items listed below were examined in the Latent Print Section.

\begin{tabular}{|c|l|}
\hline Item \# & \multicolumn{1}{|c|}{ Description } \\
\hline 1 & Demand note beginning, "I have a gun..." \\
\hline 2 & Bank of Los Angeles withdrawal form \\
\hline 3 & Pen with chain \\
\hline 4 & $\begin{array}{l}\text { Lift indicated as coming from customer } \\
\text { counter }\end{array}$ \\
\hline
\end{tabular}

Table 1: Items examined in Latent Print Section

Items of evidence submitted to the Latent Print Section for examination may be examined visually, examined with various light sources, or processed with chemicals and powders to detect the presence of latent friction ridge prints. The specific sequence of examinations and processes depends upon the nature of the evidence. ${ }^{1}$ A complete list of the processes used in this submission is maintained in the case record and can be furnished upon request.

${ }^{1}$ See Bureau of Investigation, Processing Guide for Developing Latent Prints, Revised 2001. 


\section{Results of Examinations}

Friction ridge print examinations are conducted using the Analysis, Comparison, Evaluation, and Verification (ACE-V) process. Analysis is the assessment of the quantity and quality of the information present in the print to determine if sufficient reliable details are present to conduct a comparison with another print. Comparison is the side-by-side observation of friction ridge prints to determine the extent to which the information in two prints is in agreement. Evaluation is the formulation of an opinion on the degree to which the information gathered during analysis and comparison supports the hypothesis (S) of a common source for the exemplar and the latent print or instead supports the hypothesis (D) that the exemplar and the latent come from different individuals. An opinion of strong support for $\mathrm{S}$ does not necessarily eliminate the possibility another person in the world could leave a print with areas of similar agreement. It means that within the examiner's experience and knowledge, prints from the same finger would be expected to display this much similarity, whereas prints from different fingers would not be. Verification is the independent application of the Analysis, Comparison, and Evaluation phases of the ACE-V process to a friction ridge print by another examiner.

Four latent fingerprints were detected on the items and compared to exemplar prints from THOMAS SMITH, BI \#123456, to provide evidence for or against the hypothesis that Thomas Smith is the source of the latent fingerprints.

\begin{tabular}{|c|l|c|c|}
\hline $\begin{array}{c}\text { Item } \\
\#\end{array}$ & \multicolumn{1}{|c|}{ Description } & $\begin{array}{c}\text { \# of } \\
\text { prints }\end{array}$ & $\begin{array}{c}\text { Results of comparison with } \\
\text { THOMAS SMITH, BI } \\
\text { \#123456 }\end{array}$ \\
\hline 1 & Demand note beginning, "I have a gun..." & 2 & Strong support for S \\
\hline 2 & Bank of Los Angeles withdrawal form & 1 & Strong support for D \\
\hline 3 & Pen with chain & 0 & N/A \\
\hline 4 & $\begin{array}{l}\text { Lift indicated as coming from customer } \\
\text { counter }\end{array}$ & 1 & Strong support for D \\
\hline
\end{tabular}

Table 2: Results of comparisons with THOMAS SMITH, BI \#123456

The remaining unidentified latent fingerprints were searched in the BI automated fingerprint identification system (AFIS) with the following results:

\begin{tabular}{|c|l|c|c|}
\hline $\begin{array}{c}\text { Item } \\
\#\end{array}$ & \multicolumn{1}{|c|}{ Description } & $\begin{array}{c}\text { \# of } \\
\text { prints }\end{array}$ & Results of AFIS search \\
\hline 2 & Bank of Los Angeles withdrawal form & 1 & $\begin{array}{c}\text { No support for any candidate as } \\
\text { the source }\end{array}$ \\
\hline 4 & $\begin{array}{l}\text { Lift indicated as coming from customer } \\
\text { counter }\end{array}$ & 1 & $\begin{array}{c}\text { Strong support for JANE } \\
\text { JONES, BI \#987654 as the } \\
\text { source }\end{array}$ \\
\hline
\end{tabular}

Table 3: Results of AFIS searches

The remaining unidentified latent fingerprint (on item \#2) is very different from the exemplar from JANE JONES, BI \#987654 and provides strong support for the hypothesis that she is not its source. 
The presence of a friction ridge print on an item of evidence indicates contact was made between the source and the item of evidence. The presence of a friction ridge print alone does not necessarily indicate the significance of the contact or the time frame during which the contact occurred.

Due to the many factors involved in the deposition of a friction ridge print, neither the absence of a friction ridge print on evidence nor the absence of a friction ridge print consistent with a given source necessarily precludes that source from having touched the evidence.

Blind verifications of all results were conducted by Fingerprint Analyst Robert Johnson on October 18, 2011. There were no conflicts of opinion.

Additional documentation, including bench notes, annotated images of the latent prints for both the primary analyst and verifier, and communications indicating the case information received by the analyst and the date on which that information was received are retained as part of the case record and can be provided upon request. The BI Laboratory Division's Quality Assurance Manual and Standard Operating Procedures can be found online at www.BI.org.

For questions about the content of this report, please contact Fingerprint Analyst Susanne Brown at (202) 123-4567.

The specimens are being returned under separate cover.

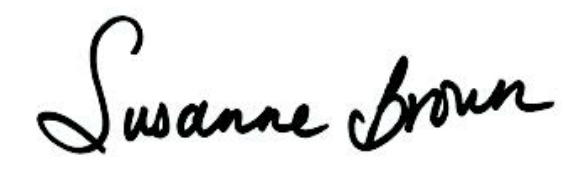

Susanne Brown

Fingerprint Analyst 


\section{Chapter 6: Testimony}

\section{Introduction and Scope}

Latent print examinations are conducted not merely to assist criminal investigators but also to produce legally admissible evidence that can exonerate or implicate defendants. Fingerprint evidence has been used in American courts since 1910. ${ }^{327}$ Other pattern and impression evidence also has a venerable history. ${ }^{328}$ In recent years, however, forensic science testimony associating marks or impressions with specific sources has come under intense scrutiny-first, in the academic literature, ${ }^{329}$ then in the courtroom, ${ }^{330}$ and again in the 2009 National Research Council (NRC) report. ${ }^{331}$

Testifying is a quintessentially human activity, and latent print testimony is the product of a long series of actions in which human factors issues are prominent. Previous chapters focus on the pretrial stages of the production of latent print evidence. They examine the role of human perception and cognition in the production of this evidence and ways to maintain or improve the quality of the resulting evidence. This chapter concentrates on the interactions of latent print examiners with other participants in the criminal justice system at and shortly before trial. Human limitations and dispositions are as important in this phase of latent print examination work as they are in earlier phases. Developing and implementing procedures and practices that encourage experts to communicate their findings accurately and fairly to lawyers, judges, and juries and to detect and correct errors in this process is a crucial component of a system that reduces the opportunities for errors in the production and presentation of courtroom fingerprint evidence. ${ }^{332}$

This chapter therefore discusses pretrial communications between experts and lawyers and makes recommendations about preparation for trial and the information that experts should communicate to judges and juries. Although the discussion is confined to presenting the results of examinations of friction skin impressions for the purpose of associating latents (anonymous,

\footnotetext{
${ }^{327}$ Mnookin, J. "Fingerprint Evidence in an Age of DNA Profiling.” Brooklyn Law Review, 67 (2001): 13.

328 Thomas, F. "Comments on the Discovery of Striation Matching and on early contributions to Forensic Firearms Identification.” Journal of Forensic Science, 12 (1967): 1 - 7.

${ }^{329}$ E.g., Faigman, D., D. Kaye, M. Saks, et al. Modern Scientific Evidence: The Law and Science of Expert Testimony. 1st ed. West Publishing Company, 1997.

${ }^{330}$ For discussions of the cases, see McCormick, C. McCormick on Evidence. Edited by Kenneth Broun. 6th ed. Thomson/West, 2006; Giannelli, P. and E. Imwinkelried. Scientific Evidence. 4th ed. Matthew Bender, 2007, Ch. 18A; Faigman, Kaye, Saks, et al., op. cit.

${ }^{331}$ National Academy of Sciences, National Research Council, Committee on Identifying the Needs of the Forensic Science Community. Strengthening Forensic Science in the United States: A Path Forward. National Academies Press, 2009, pp. 102-106.

${ }^{332}$ The Working Group was asked to examine "the role of human factors and their contributions to errors in latent print analysis." Clearly, "analysis" does not end with the Analysis phase of the Analysis, Examination, Comparison, and Verification (ACE-V) process. Nor, in the view of a substantial majority of the Working Group, does it end when a laboratory examination is completed and a report is signed. "Errors in latent print analysis" as evidence in legal proceedings can occur because of the manner in which this evidence is presented. Truncating the inquiry into the work of latent print examiners at the production of a written report would produce an incomplete review of the full process by which latent print evidence emerges from a crime scene and reaches the legal decision makers, at least in cases that go to trial.
} 
unknown prints) with exemplars (identified, known prints) in criminal trials, and differences between the civil and criminal rules of discovery are not considered, much of the discussion also applies to civil cases involving private parties.

Sections 6.1 through 6.5 describe various aspects and functions of expert testimony as well as some of the most pertinent legal doctrines. Section 6.6 discusses the admissibility and desirability of different types of testimony about the possibility that an examiner's finding that the items are (or are not) associated with one another is incorrect. Section 6.7 describes a spectrum of conceivable modes of conveying expert knowledge and findings about the possible association between a friction skin impression and its source. Finally, section 6.8 discusses quality assurance and quality control for testimony.

To situate testimony about identifications based upon the features of friction ridge skin within the broader range of forensic identification testimony, the discussion is not confined to the Analysis, Comparison, Evaluation, and Verification (ACE-V) process as currently practiced. As noted in earlier chapters, categorical conclusions within ACE-V are not the only way to make and communicate inferences from the comparison of latent prints and exemplars. In other areas of forensic science that investigate the possible association between known and unknown samples, witnesses present their findings differently, and one cannot simply assume that what is most familiar in one field is the only way to convey expert knowledge in the courtroom. This chapter therefore surveys a broad range of testimony that, at least in principle, might be provided at trial. At one pole, an expert might merely inform the judge or jury of the similarities or dissimilarities in the features of a pair of prints and not draw any inferences from these data. At the other pole, when the similarity is extensive, the expert might attribute the latent print to a specific finger (source attribution). In between these poles lie various other forms of qualitative or quantitative testimony. The Working Group reached no consensus on which one of the various alternative modes of presentation is best warranted by existing scientific research findings and other empirical knowledge. Neither does the Group purport to describe which form of testimony best satisfies the needs of the legal system. But for the reasons given in Chapter 3, the Working Group agrees that if source attributions are made, they should not be "to the exclusion of all others in the world" (Recommendation 3.7).

With respect to latent prints, two major concerns relevant to testimony are statements about error rates and individualization. This chapter does not attempt to settle the arguments among forensic scientists, psychologists, statisticians, legal scholars, and latent print examiners on fingerprint individualization and the risks of erroneous identifications or exclusions. ${ }^{333}$ Instead, it seeks to place fingerprint identification testimony in a broader context and to consider whether more modest claims of association and statements about errors would meet the law's purposes without compromising the integrity of latent print examiners and without discarding important information.

\footnotetext{
${ }^{333}$ For some assessments, see Champod, C. "Fingerprint Examination: Towards More Transparency." Law, Probability \& Risk, 7 (2008): 111; Mnookin, J. "The Validity of Latent Fingerprint Identification: Confessions of a Fingerprinting Moderate.” Law, Probability \& Risk, 7 (2008): 127.
} 


\subsection{Purpose, Form, and Preparation of Expert Testimony}

Expert witnesses use or impart specialized knowledge and information generally unknown to the jury or judge to "assist the trier of fact to understand the evidence or to determine a fact in issue ...." ${ }^{334}$ Expert testimony need not be delivered in the form of an opinion. Expert witnesses also can act as teachers, educating fact finders about pertinent principles or findings in their disciplines. For example, a latent print expert might explain why prints would not be found on a particular surface without offering a personal opinion on the reason for their absence in a particular case.

During a trial, counsel's questioning frames the expert's presentation. The expert may not simply decide what information to discuss but must answer counsel's questions. This can create some tension between the goal of being complete and the need to be responsive. In resolving this tension, "ethical considerations and professional standards properly place a number of constraints on the expert's behavior." ${ }_{335}$ One such constraint is "a requirement of candor. While an expert is ordinarily under no legal obligation to volunteer information, professional ethics may compel this ... when the expert believes that withholding information will change dramatically the picture that his ... analyses, properly understood, convey." 336 Thus, the "guiding principles" proposed by the American Society of Crime Laboratory Directors/Laboratory Accreditation Board (ASCLD/ $\mathrm{LAB}$ ) advise forensic scientists to "attempt to qualify their responses while testifying when asked a question with the requirement that a simple 'yes' or 'no' answer be given, if answering 'yes' or 'no' would be misleading to the judge or the jury." 337

Pretrial preparation with the attorney who will be calling the expert is essential to effective trial presentation. Preparation includes educating the lawyer, who should understand the evidence involved, appreciating the limitations of the discipline and the forensic findings, and recognizing any exculpatory or qualifying information that must be disclosed. Lawyers should not present the testimony of examiners without first conferring about the expert's report and anticipated testimony, including the use of visual aids. ${ }^{338}$

\section{Recommendation 6.1: The trial preparation process should address the presentation of technical information in lay terms, the organization of the direct examination, possible cross-examination, and the possible use of visual aids.}

${ }^{334}$ Federal Rules of Evidence 702. In full, the rule states that: If scientific, technical, or other specialized knowledge will assist the trier of fact to understand the evidence or to determine a fact in issue, a witness qualified as an expert by knowledge, skill, experience, training, or education, may testify thereto in the form of an opinion or otherwise, if (1) the testimony is based upon sufficient facts or data, (2) the testimony is the product of reliable principles and methods, and (3) the witness has applied the principles and methods reliably to the facts of the case.

For more extensive discussions of expert testimony, see, for example, Faigman, Kaye, Saks, et al., op. cit.; Giannelli and Imwinkelried, op. cit.; Kaye, D., D. Bernstein, and J. Mnookin. The New Wigmore, A Treatise on Evidence: Expert Evidence. 2nd ed. Aspen Publishers, 2011; McCormick, op. cit.

${ }^{335}$ Feinberg, S., ed. The Evolving Role of Statistical Assessments as Evidence in the Courts. Springer, 1989, p. 161.

${ }^{336} \mathrm{Ibid}$.

${ }^{337}$ American Society of Crime Laboratory Directors/Laboratory Accreditation Board. ASCLD/LAB Guiding

Principles of Professional Responsibility for Crime Laboratories and Forensic Scientists. Version 1.1. 2009, Principle 19.

${ }^{338}$ Using pictures of prints not involved in the case should not be necessary and may be objectionable under the rules of relevance. 


\subsection{Pretrial Discovery}

There is no legal barrier to defense counsel contacting a prosecution expert to discuss the case. ${ }^{339}$ Of course, the mere fact that opposing counsel may contact an expert does not require the expert to cooperate, and there may be concerns about breaching the protection given to an attorney's work product. However, openness may help ensure that opposing counsel understands the discipline, the evidence, and the opinion. It also enhances credibility if the expert on the witness stand discloses sharing information with opposing counsel.

Different considerations apply to a criminal defense expert. Unless and until a defense expert is designated a testifying witness, this expert may be considered part of the defense team, and defense counsel needs to protect confidential communications that come from clients. ${ }^{340}$ Once designated as a witness, however, this expert should have the same status as a prosecution expert.

\subsection{Ethical and Professional Obligations}

An expert witness who is a member of a learned profession has obligations to his profession as well as to his employer. ${ }^{341}$ More than 30 years ago, an NRC panel called on "professional organizations to develop standards for expert witnesses in legal proceedings ...." "342 More recently, the NRC committee on forensic science proposed "a national code of ethics for all forensic science disciplines" such that "those forensic scientists who commit serious ethical violations" could be sanctioned "through a certification process for forensic scientists." 343 It observed that

many forensic science organizations - such as the American Academy of Forensic Sciences, the California Association of Criminalists, and ASCLD [American Society of Crime Laboratory Directors] — have codes of ethics or codes of professional practice imploring members to act with honesty, integrity, and objectivity; to work within the bounds of their professional competence; to present testimony and reports in a clear and objective manner; and to avoid conflicts of interest and potential bias, among other things. ${ }^{344}$

To avoid incomplete or one-sided presentations, ample disclosure of reasoning, limitations, and exculpatory material in pretrial reports, as discussed in section 6.1, is especially important, as is a shared professional ethic that is strong enough to resist pressure from lawyers who sometimes seek unduly oversimplified and exaggerated presentations of technical information. Thus, forensic scientists have urged that

\footnotetext{
${ }^{339}$ Gross, S. "Expert Evidence.” Wisconsin Law Review, 1113 (1991): 1151.

${ }^{340}$ United States v. Tharpe, 38 M.J. 8, 28 (C.M.A. 1993).

${ }^{341}$ Feinberg, op. cit., pp. 161-62; Kaye, Bernstein, and Mnookin, op. cit., § 11.4(c); Lubet, S. "Expert Witnesses: Ethics and Professionalism." Georgetown Journal of Legal Ethics, 12 (1999): 465.

${ }^{342}$ Feinberg, op. cit., p. 162.

${ }^{343}$ NAS, NRC, Committee on Identifying the Needs of the Forensic Science Community, op. cit., p. 214.

${ }^{344}$ Ibid.
} 
all crime laboratories, both public and private, should have a code of ethics/ conduct. These codes need to stress the best interests of society through government service and justice, government responsibility for professionalism and cost effectiveness, integrity (both professionally and regarding evidence), objectivity, staying within the bounds or limits of what the science can provide, maintaining confidentiality, complying to legal demands such as disclosure, and being truthful. ${ }^{345}$

In view of the status of experts who represent a learned and skilled profession and the latitude given to the form and bases of their testimony (see section 6.1), the Working Group recommends:

\section{Recommendation 6.2: Forensic service providers should adopt codes of ethics that require testifying in a nonpartisan manner; answering questions from both the prosecution and the defense directly, accurately, and fully; and providing appropriate scientific information before, during, and after trial.}

This precept already is widely accepted in the forensic community. The Scientific Working Group on Friction Ridge Analysis, Study and Technology (SWGFAST) has a short aspirational code for friction ridge examiners that forbids knowing misrepresentations and specifies that "testimony shall be rendered in an impartial manner to promote the understanding of examinations and findings." 346 The guiding principles of ASCLD/LAB specifically state that "wording should not be such that inferences may be drawn which are not valid, or that slant the opinion to a particular direction." ${ }^{347}$ As previously noted, to achieve impartial testimony, ASCLD/ LAB also advises forensic scientists to "attempt to qualify their responses while testifying when asked a question with the requirement that a simple 'yes' or 'no' answer be given, if answering 'yes' or 'no' would be misleading to the judge or the jury." 348 The European Network of Forensic Science Institutes expects analysts to "deal with questions truthfully, impartially and flexibly in a language which is concise, unambiguous and admissible;" to "give explanations to specific questions in a manner that facilitates understanding by nonscientists;" to "consider additional information and alternative hypotheses that are presented to you;" to "consider and evaluate these and express relevant opinions taking into account the limitations on opinions which cannot be given without further examination and investigation;" and to "clearly differentiate between fact and opinion and ensure that the opinions you express are within your area of expertise." 349

Notwithstanding the valuable role that professional organizations can play in defining and enforcing ethical requirements, it must be noted that professional organizations can restrict

\footnotetext{
${ }^{345}$ Budowle, B., M. Bottrell, S. Bunch, et al. "A Perspective on Errors, Bias, and Interpretation in the Forensic Sciences and Direction for Continuing Advancement.” Journal of Forensic Sciences, 54 (July 2009 ): 803.

${ }^{346}$ Scientific Working Group on Friction Ridge Analysis, Study and Technology. A Model Policy for Friction Ridge Examiner Professional Conduct. Version 1.0. Scientific Working Group on Friction Ridge Analysis, Study and Technology, December 2008.

${ }^{347}$ ASCLD/LAB, op. cit., Principle 18.

${ }^{348}$ Ibid., Principle 19.

${ }^{349}$ European Network of Forensic Science Institutes Standing Committee for Quality and Competence. Performance Based Standards for Forensic Science Practitioners. European Network of Forensic Science Institutes Standing Committee for Quality and Competence, July 2004.
} 
ethically acceptable testimony as well as ethically dubious conduct. As noted in Chapters 1 and 3, the International Association for Identification (IAI) passed a resolution in 1979 making it professional misconduct for any latent print examiner to provide courtroom testimony that labeled an identification "possible, probable, or likely," rather than "certain." "350 Yet, the insistence on subjective certainty could produce a threshold that is too high — one that excludes testimony of an association that is less certain but still very useful to the trier of fact. Indeed, this norm has prompted the criticism that "fingerprint examiners are now unique among forensic analysts in that they are ethically bound to frame their conclusion in terms of absolute certainty." 351

In 2010, IAI rescinded its 1979 resolution as "not consistent with advancements since [its] passage." "352 The new resolution states "that Resolution 1979-7 and Resolution 1980-5 are hereby rescinded" and approves of "a clear and unambiguous presentation" of "associations based on ... class characteristics (pattern type, ridge flow)." Whether the IAI will interpret this rescission as allowing its members to testify to degrees of confidence short of subjective certainty remains to be seen. Presumably, the "advancements" are "mathematically based models to assess the associative value of the evidence [that] may provide a scientifically sound basis for supporting the examiner's opinion." 353 The resolution prevents an examiner from using a model that has not "been accepted as valid by the IAI" and warns that the "use of mathematically based models does not relieve the examiner of responsibility for their expert opinion.." 354 Chapter 4 also discusses the importance of validating models and the need for the government to be open with data for validation studies.

\subsection{Expressions of Certainty}

In Daubert v. Merrell Dow Pharmaceuticals, Inc., ${ }^{355}$ the Supreme Court observed that "it would be unreasonable to conclude that the subject of scientific testimony must be 'known' to a certainty; arguably, there are no certainties in science." ${ }^{356}$ Thus, courts do not normally demand absolute certainty from scientists (or any other experts), but they do require more than conjecture. They exclude expert opinions that are so weak or speculative that they would not be helpful to a jury relative to the time they could consume and the misunderstandings they might generate. This is a special application of the broad principle that the trial judge should exclude even relevant evidence when "its probative value is substantially outweighed by the danger of

\footnotetext{
350 International Association for Identification. "Resolution VII." Identification News, 29 (August 1979). The next year the resolution was amended to make it clear that it applied only to examiners who made a probabilistic identification on their own initiative (rather than, say, under threat of a contempt citation). See International Association for Identification. "Resolution VII Amended." Identification News, 3 (August 1980). Yet, analysts have departed from this constraint by testifying to possible matches. E.g., Warney v. Monroe County, 587 F.3d 113,118 (2d Cir. 2009).

${ }^{351}$ Cole, S. "Fingerprinting: The First Junk Science?" Oklahoma City University Law Review, 28, no. 73 (2003): 88.

${ }^{352}$ International Association for Identification. IAI Resolution 2010-18. International Association for Identification, July 16, 2010.

353 Ibid.

354 Ibid.

355509 U.S. 579 (1993).

${ }^{356}$ Ibid., p. 590.
} 
unfair prejudice, confusion of the issues, or misleading the jury, or by considerations of undue delay, waste of time, or needless presentation of cumulative evidence." 357

In the medical discipline, to signal that an opinion was sufficiently definitive to be helpful, the practice of having physicians testify to diagnoses or other opinions in terms of "a reasonable degree of medical certainty" developed in many jurisdictions. ${ }^{358}$ In time, this practice spread to other professions and has led to scientists testifying "to a reasonable degree of scientific certainty." 359

Outside the courtroom, however, scientists do not communicate their findings in this fashion. An astronomer who reports the discovery of an exoplanet does not characterize the finding as satisfying some "reasonable degree of scientific certainty." A chemist who deduces the identity of a compound from its nuclear magnetic resonance spectrum has no table of degrees of scientific certainty with which to label the deduction. Scientists might refer to personal degrees of confidence in a finding or to the degree of controversy surrounding it, but there is no generally accepted or working definition of a "reasonable degree of certainty" in scientific discourse. ${ }^{360}$

The dissonance between the legal phrase and the scientific practice creates an issue for latent print examiners and forensic scientists of all types who are asked to use this phrase. When meeting with the attorney before trial, an expert not only should inform the attorney of the conclusion but also should specify the degree of confidence that can be expressed at trial. For example, an expert might explain to the attorney, and later in court, that although the discipline does not normally use the phrase "reasonable degree of scientific certainty," he is very confident in his conclusions based on his experience and the standards of the field. It is the lawyer's responsibility to advocate for the admission of such testimony. Although individual trial judges could be skeptical of admitting testimony without the familiar accompanying words, it is clear that no rule of evidence requires the use of a phrase such as "reasonable degree of scientific certainty." 361 To the contrary, modern courts would not "expect dogmatic diagnoses from a careful scientist." ${ }^{\prime 32}$

\footnotetext{
${ }^{357}$ Federal Rules of Evidence 403.

${ }^{358}$ For extreme examples of such testimony, see Cooey v. Strickland, 589 F.3d 210, 231 (6th Cir. 2009) (testimony "beyond a reasonable degree of medical certainty"); Lewicki v. Longshore, No. 17004/06, 2009 WL 4667411, at 2 (N.Y. Sup. Ct. Dec. 7, 2009).

${ }^{359}$ See Kaye, D. The Double Helix and the Law of Evidence. Harvard University Press, 2010.

${ }^{360}$ In the social sciences and some other fields, it is conventional to regard a " $p$-value" of 0.05 as necessary to report a "statistically significant" finding. But this p-value is not a measure of the probability that the "null hypothesis" is true. Although the point is not always appreciated in the courtroom, the $p$-value refers to the probability of the data assuming that the hypothesis is true. See Kaye, D. and D. Freedman. "Reference Guide on Statistics." In Reference Manual on Scientific Evidence edited by Committee on the Development of the Third Edition of the Reference Manual on Scientific Evidence and Committee on Science, Technology, and Law Policy and Global Affairs, 3d edition, pp. 211 - 302. National Academies Press, 2011. Consequently, it is not correct to say that one can have 95\% confidence in the alternative hypothesis just because $p<0.05$. That said, the similarities (or dissimilarities) in a pair of prints that would lead an examiner to declare an identification (or an exclusion) should be so extensive or clear as to produce a $p$-value that is considerably less than the conventional level of 0.05 .

${ }^{361}$ E.g., United States v. Willock, 696 F.Supp.2d 536, 574 (D.Md. 2010); United States v. Glynn, 578 F.Supp.2d 567, 574 (S.D.N.Y. 2008).

${ }^{362}$ Amax Coal Co. v. Beasley, 957 F.2d 324, 328 (7th Cir.1992).
} 
Furthermore, even if a scientist is comfortable with the phrase, problems can arise. Some courts have rejected such testimony as an antidote to what they have deemed to be unjustifiably strong claims of identity. For example, some courts have concluded that the scientific foundation for absolute identification of toolmarks is insecure, but they still treat the matches discerned by skilled examiners as highly probative. This line of authority rejects statements of unique identification "as a matter of scientific certainty," 363 but permits it "within a reasonable degree of certainty in the firearms examination field," "364 "to a reasonable degree of ballistic certainty," ${ }^{365}$ or only as being "more likely than not." 366 Similar reasoning has been applied to exclude assertions of scientific certainty for latent fingerprint identification testimony, ${ }^{367}$ although it remains to be seen whether such restrictions will displace the widely accepted practice of allowing expressions of absolute confidence in source attributions.

\subsection{Reliability, Validity, General Acceptance, and Prejudice}

The courts that have constrained expressions of certainty about holistic pattern matching have done so to avoid outright exclusion of the evidence. Because scientific evidence might overly impress a jury, it is held to higher standards for admissibility than most other expert testimony. ${ }^{368}$ These are the "general acceptance" and scientific "reliability" standards. ${ }^{369}$ The general acceptance standard originated in the 1923 case of Frye v. United States. ${ }^{370}$ In Frye, the U.S. Court of Appeals for the District of Columbia Circuit upheld the exclusion of testimony from a psychologist who used a blood-pressure cuff to determine that a defendant's denial of a murder to which he had previously confessed was not the result of conscious deception. The Court of Appeals stated that

just when a scientific principle or discovery crosses the line between the experimental and demonstrable stages is difficult to define. ... While courts will go a long way in admitting expert testimony deduced from a well-recognized scientific principle or discovery, the thing from which the deduction is made must be sufficiently established to have gained general acceptance in the particular field in which it belongs. ${ }^{371}$

\footnotetext{
${ }^{363}$ United States v. Taylor, 663 F.Supp.2d 1170, 1180 (D.N.M. 2009).

364 Ibid.

${ }^{365}$ United States v. Monteiro, 407 F.Supp.2d 351, 375 (D.Mass. 2006); Commonwealth v. Pytou Heang, 942 N.E.2d 927 (Mass. 2011).

366 United States v. Glynn, 578 F.Supp.2d 567, 574-75 (S.D.N.Y. 2008).

${ }^{367}$ United States v. Zajac, No. 2:06-cr-00811-CW (D. Utah Sept. 16, 2010).

${ }^{368}$ Kaye, Bernstein, and Mnookin, op. cit., $\$ 8.1$.

${ }^{369}$ Expert testimony generally is subject to a "reliability" inquiry in many jurisdictions because of the frequently partisan nature of expert testimony in the adversary system and the limited competence of judges and juries to evaluate expert reasoning. Kaye, Bernstein, and Mnookin, op. cit., Ch.1. "Reliability" here should not be confused with reliability in the statistical or scientific sense. Evidentiary reliability refers to a measurement process that is valid (it measures the quantity of interest), reliable (repeated measurements have low variance), and accurate (the measurements are close to the true values). Often, accuracy is considered an aspect of validity. E.g., Haber, L. and R. Haber. "Scientific Validation of Fingerprint Evidence Under Daubert." Law, Probability \& Risk, 7 (2008): 87. Whatever terms are used, such a process produces evidence that can be relied on.

370293 F. 1013 (D.C. Cir. 1923).

${ }^{371}$ Ibid. p. 1014.
} 
Over the years, Frye became the dominant test for scientific expert testimony in the United States. ${ }^{372}$ In the 1970 s or so, however, an alternative standard that simply required scientific evidence to be especially reliable emerged in a significant minority of jurisdictions. ${ }^{373}$

In 1993, the Supreme Court confirmed and accelerated this trend. In Daubert v. Merrell Dow Pharmaceuticals, Inc., ${ }^{374}$ plaintiffs alleged that a drug was a teratogen, but the Court of Appeals held that this theory lacked general acceptance because no published, peer-reviewed epidemiological studies demonstrated a statistically significant association between the drug and the type of birth defects experienced by the plaintiffs' children. The Supreme Court held that, although the 1975 Federal Rules of Evidence were silent about whether they were perpetuating or abandoning Frye, they implicitly repudiated general acceptance as the sole requirement for scientific evidence. The Court wrote that while trial judges could continue to consider whether existing research has produced general scientific acceptance, this factor is not necessarily dispositive. Rather, the trial court should assess such additional factors as the extent to which the theory had been tested, the content of peer-reviewed publications, the existence of controlling standards in applying the technique, and the known error rate of the system. ${ }^{375}$ Only if this wide-ranging inquiry revealed that the theory and its implementation were "reliable" would the evidence satisfy Rule 702. Many state courts now apply the Daubert standard, but the Frye standard remains intact in other states.

Finally, in 1999, the Supreme Court addressed a question left open in Daubert: whether nonscientific expert evidence was to be assessed with the factors articulated for the testimony of physicians, toxicologists, and epidemiologists at issue in Daubert itself. In Kumho Tire Co. v. Carmichael, ${ }^{376}$ the Court upheld the District Court's exclusion of an engineer's determination that a tire blew out because of a manufacturing defect as resting on an inadequately validated form of "visual and tactile inspection." In doing so, it squarely rejected the Court of Appeals for the Eleventh Circuit's view that "a Daubert analysis" applies only where an expert relies "on the application of scientific principles," rather than "on skill- or experience-based observation." 377 The Kumho Court held that the trial court had the discretion to evaluate technical or experiential expert testimony in light of the factors listed in Daubert, but it added that "the test of reliability is 'flexible,' and Daubert's list of specific factors neither necessarily nor exclusively applies to all experts or in every case." ${ }^{\prime 37}$ The Court explained that

Daubert's general holding — setting forth the trial judge's general 'gatekeeping' obligation - applies not only to testimony based on "scientific" knowledge, but also to testimony based on 'technical' and 'other specialized' knowledge. See Fed. Rule Evid. 702. We also conclude that a trial court may consider one or more of the more specific factors that Daubert mentioned when doing so will help determine that testimony's reliability. But, as the Court stated in Daubert, the test

\footnotetext{
${ }^{372}$ Kaye, Bernstein, and Mnookin, op. cit., $\S 6.3$.

${ }^{373}$ Ibid., § 7.2.

374509 U.S. 579 (1993).

${ }^{375}$ Additional factors, such as whether the expert testing was done expressly for litigation, are enumerated in Kaye, Bernstein, and Mnookin, op. cit.

376526 U.S. 137, 154 (1999).

${ }^{377}$ Carmichael v. Samyang Tire, Inc., 131 F.3d 1433, 1435-36 (1997).

378526 U.S. 141.
}

121 Latent Print Examination and Human Factors: Improving the Practice through a Systems Approach The Report of the Expert Working Group on Human Factors in Latent Print Analysis 
of reliability is 'flexible,' and Daubert's list of specific factors neither necessarily nor exclusively applies to all experts or in every case. ${ }^{379}$

Daubert inspired many attacks on identification procedures that police laboratories routinely performed and that criminal courts universally accepted. If these procedures were held to the standards required for the approval of new drugs, or for a consensus among epidemiologists that a substance is a human carcinogen, it seemed that they might be tossed out of court. Latent fingerprint identification, long considered the gold standard for forensic identification techniques, was not immune from these challenges. In fact, in one noted case, a federal district judge - and a former dean of the law schools at the University of Pennsylvania and Yale University-ruled that because the ACE-V process failed to meet the Daubert test for scientific evidence, an examiner could not testify to an individualization (but could fulfill the teaching function of an expert by demonstrating to the jury the remarkable congruence in the images being compared); however, the court promptly reconsidered and vacated the order, reasoning that fingerprint testimony was as good as many other forms of skilled expert testimony. ${ }^{380}$ Judge Pollak wrote that

I am not persuaded that courts should defer admission of testimony with respect to fingerprinting — ... 'the bedrock forensic identifier of the 20th century' — until academic investigators ... have made substantial headway on a 'verification and validation' research agenda. Such research would be all to the good. But to postpone present in-court utilization of this 'bedrock forensic identifier' pending such research would be to make the best the enemy of the good. ${ }^{381}$

In other words, the judge emphasized the "flexibility" of Kumho over the earlier search for more extensive scientific validation.

More recently, as noted in section 6.4, a small number of courts have curtailed the certainty with which a judgment of individualization of toolmarks may be expressed. They reason that enough scientific research and experience has been accumulated to establish the value of holistic pattern matching of toolmarks as a contribution of skilled witnesses, but these courts do not allow the prosecution or the expert witness to portray an individualization as the product of a rigorous, scientific technique. ${ }^{382}$

By insisting that holistic comparisons are rigorous scientific experiments, practitioners have invited challenges based upon the most demanding legal standards. Many latent print examiners are prone to emphasize that they follow the "scientific method." A Federal Bureau of Investigation (FBI) publication, for example, maintains that "the ACE-V ... methodology, is a series of steps found within the scientific method," 383 and SWGFAST defines ACE-V as "the

\footnotetext{
${ }^{379}$ Ibid.

${ }^{380}$ United States v. Llera Plaza, Crim. No. 98-362-10, 11, 12, 2002 U.S. Dist. LEXIS 344 (E.D. Pa. Jan. 7, 2002), vacated, 188 F. Supp. 2d 549 (E.D. Pa. 2002).

${ }^{381}$ Ibid., p. 572.

${ }^{382}$ E.g., United States v. Willock, 696 F.Supp.2d 536 (D.Md. 2010); United States v. Taylor, 663 F.Supp.2d 1170 , 1180 (D.N.M. 2009); Commonwealth v. Pytou Heang, 942 N.E.2d 927 (Mass. 2011).

383 Peterson, P., C. Dreyfus, M. Gische, et al. "Latent Prints: A Perspective on the State of the Science." Forensic Science Communications, 11, no. 4 (October 2009). See also Cole, S. "Comment on Scientific Validation of Fingerprint Evidence Under Daubert.” Law, Probability \& Risk, 7, no. 119 (2008): 123 (collecting similar statements).
} 
acronym for a scientific method." 384 Naturally, some witnesses express this view in their testimony. In State v. Foreman, ${ }^{385}$ a latent print examiner from Connecticut's state forensic laboratory with "extensive qualifications" testified that "our comparisons are conducted through a scientific methodology known as ACE-V." ${ }^{386}$ During cross-examination, he reiterated that "based on our training, education, experience, based on fact and history of fingerprints, fingerprints is a science. As I explained earlier, the comparison methodology using ACE-V methodology is a scientific methodology." 387

As we have seen, however, when confronted with serious controversy over the scientific nature of inferences from a technique or procedure that is portrayed or perceived as highly scientific, the proponent of the evidence must demonstrate sufficient scientific studies to establish the technique's validity or its general acceptance (depending on the jurisdiction). When the process is more intuitively accessible and is not heavily wrapped in the rhetoric of science, courts dispense with a showing of scientific validation or general scientific acceptance. ${ }^{388}$ In particular, courts impressed with the Daubert objections to toolmark and fingerprint identification have emphasized the flexibility provided by Kumho to admit the evidence, at least where the expert does not attest to scientific certainty. ${ }^{389}$ Testimony that the evaluation process is strictly scientific or produces scientifically certain results thus undermines the theory that some courts have used to overcome Rule 702 objections.

Proponents of expert testimony may need to be aware of two other issues. First, evidence must be such that its probative value is not substantially outweighed by its prejudicial effect. ${ }^{390}$ The proponent must also assess whether a debate over how to label the expert's approach - as a "scientific" method - could divert the jury's attention from the adequacy of the examiner's conclusions in the particular case. As noted in Chapters 1 and 3, ACE-V maps the steps of a process, but it does not provide specific functional guidance on how to carry out that process nor does it detail what the substantive content of those various steps should be. As a result, the legal and scientific literature contains assertions that "merely following the steps of ACE-V does not imply that one is proceeding in a scientific manner," 391 that the invocation of the scientific

\footnotetext{
${ }^{384}$ Scientific Working Group on Friction Ridge Analysis, Study and Technology. Glossary, Version 2.0. Scientific Working Group on Friction Ridge Analysis, Study and Technology, May 2009.

385 State v. Foreman, 954 A.2d 135 (Conn. 2008).

${ }^{386}$ Ibid., p. 155 , note 19.

${ }^{387}$ Ibid.

${ }^{388}$ E.g., Ex parte Dolvin, 391 So.2d 677, 679 (Ala. 1980); People v. Clark, 857 P.2d 1099 (Cal. 1993); State v. Reid, 757 A.2d 482, 487 (Conn. 2000); Carter v. State, 766 N.E.2d 377 (Ind. 2002). See generally Kaye, Bernstein, and Mnookin, op cit:; McCormick, op. cit.

${ }^{389}$ See, e.g., United States v. Baines, 573 F.3d 979, 992 (10th Cir. 2009); United States v. Llera Plaza, Crim. No. $98-$ 362-10, 11, 12, 2002 U.S. Dist. LEXIS 344 (E.D. Pa. Jan. 7, 2002), vacated, 188 F. Supp. 2 d 549 (E.D. Pa. 2002 ). Compare United States v. Gutierrez-Castro, 86 Fed. R. Evid. Serv. 319 (D.N.M. 2011) (allowing an examiner “to testify about the methods and practices of inked fingerprint analysis, and to ... testify that all the fingerprints belong to the same person," but ordering that "[t]he United States may not, ... offer [him] as an expert witness in the jury's presence, the Court will not certify [him] as an expert witness in the jury's presence, and the jury instructions will not refer to [him] as an expert witness").

${ }^{390}$ See Federal Rules of Evidence 403; McCormick, op. cit.

${ }^{391}$ NAS, NRC, Committee on Identifying the Needs of the Forensic Science Community, op. cit., p. 142.
} 
method is only an "analogy," 392 that "ACE-V is an acronym, not a methodology," 393 that it is "a broadly stated framework" 394 better described as "common sense," 395 and that "the ACE-V protocol remains obscure" 396 because it rests on "an ill-defined, flexible, and explicitly subjective criterion." 397 Such statements are listed not to endorse them and not to decide the semantic or philosophical question of whether ACE-V is scientific. ${ }^{398}$ Instead, these statements suggest that objections based on the premise that it is prejudicial to describe ACE-V as "science" can be avoided by referring to ACE-V as a systematic and widely used process for determining whether two patterns have a common origin.

Second, advances in computer pattern matching for fingerprint identification can be expected to come before the courts, and these surely will have to be evaluated under the Frye or Daubert standard for pure scientific evidence. For example, as explained in Chapter 4, automated systems can measure the distance (in an abstract mathematical space) between certain features in two images. They can generate probabilities for such distances under the assumption that the image of the unknown print comes from a finger known to have produced an exemplar and that it came from a finger of a randomly selected individual represented in a reference set of exemplars from many individuals. Such conditional probabilities (also known as "likelihoods"), if they rest on adequate data and valid models, might be used in their own right or to reinforce (or contradict) the judgments of a human examiner. Before this can happen in a jurisdiction that applies the general acceptance standard, scientific studies validating the automated system must convince the scientific community that it is reliable and accurate for this particular use. In jurisdictions that follow Daubert, a court should assess factors such as the extent of peer-reviewed publications and the known error rate of the system to assure itself that the theory and its implementation are scientifically sound.

\subsection{Errors and Error Rates}

Chapter 2 describes the major types of errors in examining two impressions to determine whether they came from the same source. It notes cases in which expert witnesses assured courts that the ACE-V process, if followed faithfully, could not err, and it covers the resulting criticisms from commentators, courts, and even within the latent print community. Assertions that false identifications occur at a rate of less than one in a million comparisons have not fared well either. Precise estimates of error rates, based on empirical research with a large number of cases in which ground truth was established, were not available. Yet, virtually all courts accepted expert testimony that fingerprint identifications and exclusions were absolutely certain to be correct.

In several other fields, where controlled experiments or field studies have been conducted, many court opinions treat the observed error rates of scientific tests as a consideration affecting the admissibility of these tests or the weight to be given to the results in a particular case. For

\footnotetext{
${ }^{392}$ Haber and Haber, op. cit., p. 98.

393 Zabell, S. "Fingerprint Evidence.” Journal of Law and Policy, 13 (2005): 143.

${ }^{394}$ NAS, NRC, Committee on Identifying the Needs of the Forensic Science Community, op. cit., p. 142.

${ }^{395}$ Zabell, op. cit., p. 178.

${ }^{396}$ C. Champod, op. cit., p. 114.

${ }^{397}$ Stoney, D. "Measurement of Fingerprint Individuality." In Advances in Fingerprint Technology, edited by Henry

C. Lee and R.E. Gaensslen, 327-87. 2nd ed. CRC Press, 2001.

${ }^{398}$ On this issue, see Cole, 2008, op. cit.
} 
example, a significant body of controlled experiments and field studies of the accuracy of polygraphic lie detection has informed opinions on the admissibility of this type of evidence. ${ }^{399}$ A smaller set of laboratory experiments of visual spectrographic speaker identification also is available. ${ }^{400}$

Until very recently, almost no experimental studies of the accuracy of the latent print examination process existed. ${ }^{401}$ In 2011, the results of a major experiment, known as the NoblisFBI experiment, were published. ${ }^{402}$ Because of the size and careful design and execution of the study, it is sure to play a role in litigation on the admissibility of latent print evidence. As explained in Chapter 2 (Box 2.4), 169 latent print examiners each were presented with 100 pairs of latent and exemplar prints. In the more than 10,000 presentations deemed of value for individualization, $97 \%$ of the examiners made no false identifications; the remaining 5 examiners made a total of 6 false identifications, for a false positive rate of about $0.1 \%$. The examiners made 450 false exclusions for prints deemed of value for identification or exclusion, for a false negative rate of about $7.5 \% .{ }^{403}$ However, the examiners who volunteered for the experiment were not a random sample of all examiners, and although the latent prints were chosen to be representative of casework, the pairings as a group could have been more (or less) challenging than typical casework. Thus, there is room for additional research on errors and for caution in applying the measurements of error rates in this study to the performance of particular examiners in specific cases.

Some observers have proposed using existing proficiency test results (or more rigorous, blind tests) to produce estimates of or bounds on error rates for a particular examiner, for a given laboratory, or for the field as a whole. ${ }^{404}$ As noted in Chapter 3, whether this procedure can produce reasonably precise estimates of the probability of a false positive or negative in a particular case is questionable. The issue is hardly new. ${ }^{405}$ Although the 2009 NRC report is silent about the value of proficiency test results as an indication of case-specific error rates, two

\footnotetext{
${ }^{399}$ For a review, see National Academy of Sciences, National Research Council, Committee to Review the Scientific Evidence on the Polygraph, The Polygraph and Lie Detection, 2003.

${ }^{400}$ For reviews, see Bolt, R., F. Cooper, E. David, et al. "Speaker Identification by Speech Spectrographs: Some Further Observations." Journal of the Acoustical Society of America, 54 (1973): 531; Kent, R. and M. Chial. "Talker Identification: Scientific Status." In Modern Scientific Evidence: The Law and Science of Expert Testimony edited by Faigman, D., M. Saks, J. Sanders, et al., vol. 1, pp. 113 - 151. Thomson Reuters/West, 2009.

${ }^{401}$ Limited data come from the United Kingdom (Evett, I. and R. Williams. "A Review of the Sixteen Points Fingerprint Standard in England and Wales." Journal of Forensic Identification, 46, no. 1 (1996): 49), from proficiency tests in Australia (Gutowski, S. "Error Rates in Fingerprint Examination: The View in 2006." The Forensic Bulletin, Autumn 2006, 18), and from a study of six examiners in Minnesota (Langenburg, G. "A Performance Study of the ACE-V Process: A Pilot Study to Measure the Accuracy, Precision, Reproducibility, Repeatability, and Biasability of Conclusions Resulting from the ACE-V Process." Journal of Forensic Identification, 59, no. 2 (2009): 219 - 57).

${ }^{402}$ Ulery, B., A. Hicklin, J. Buscaglia, et al. "Accuracy and Reliability of Forensic Latent Fingerprint Decisions." Proceedings of the NAS. 2011.

${ }^{403}$ Not counting outcomes of "inconclusive," the figures are $0.2 \%$ and $10.2 \%$, respectively. See Chapter 2 (Table 2.9).

${ }^{404}$ E.g., Koehler, J. "Fingerprint Error Rates and Proficiency Tests: What They Are and Why They Matter." Hastings Law Journal, 59 (2008): 1077; Teichroeb, R. "Produce Crime Lab Error Rates, Some Urge." Seattle PostIntelligencer, July 21, 2004. Accessed November 28, 2011. http://www.seattlepi.com/default/article/Produce-crimelab-error-rates-some-urge-1149873.php.

${ }^{405}$ E.g., Berger, M. "Laboratory Error Seen Through the Lens of Science and Policy." University of California Davis Law Review, 30 (1997): 1081.
} 
previous NRC reports on DNA evidence discuss the topic. A 1992 committee favored the production of proficiency test results as trial evidence. ${ }^{406}$ A 1996 committee did not go this far, but it supported the disclosure of test results in reports. ${ }^{407}$ Building on these ideas, defendants have argued that evidence of a DNA match should be inadmissible unless this information accompanied it. The courts consistently rejected the argument. ${ }^{408}$

A defendant might seek to introduce statistics to show that examiners as a group do not have perfect scores on proficiency tests or that the specific witness has made false positive or negative findings in the past. ${ }^{409}$ In response to this defensive use of error rates, it has been argued that the use of an average statistic is "not meaningful" because not all examiners are equal and that "cumulative error over time is not a meaningful mechanism for assessing current error rate" in that steps inevitably will be taken to correct errors. ${ }^{410}$

Both statements are problematic. First, the mean is generally a reasonable estimator of the scores of the members of a group. If other information on individuals is available, adjustments can be made to the mean for those individuals. ${ }^{411}$ If no other information is available, using the sample mean is still more accurate than random guessing. Second, even if a process is improving over time, using a recent average is likely to be more accurate than random guessing. Therefore, the statistics cannot be dismissed as meaningless, but their utility remains debatable. As one group of latent print examiners argue,

calculating human error rates is a complex undertaking, because unlike instruments, human beings change, learn, and adapt, particularly when faced with errors and the quality assurance systems designed to overcome these errors and improve practices. As a result, the chance that human errors will be made or repeated is constantly changing. ${ }^{412}$

No published appellate opinions seem to have considered the admissibility of error-rate statistics under the principles of Federal Rules of Evidence 403 and $404 .{ }^{413}$ In Williams v. State, ${ }^{414}$ Maryland's highest court reversed a conviction because "the trial judge erred in restricting Williams from fully cross examining [the DNA analyst] concerning the prevalence of testing errors and contamination during PCR [polymerase chain reaction] testing at Cellmark." ${ }^{15}$ The

\footnotetext{
${ }^{406}$ National Academy of Sciences, National Research Council, Committee on DNA Technology in Forensic Science. DNA Technology in Forensic Science. National Academies Press, 1992.

${ }^{407}$ See Kaye, The Double Helix and the Law of Evidence, 2010, op. cit., pp. 162-170.

${ }^{408}$ Ibid.

${ }^{409}$ Six out of the one hundred sixty-three examiners who responded to questions about "ever having made an erroneous individualization (after training)" in the Noblis-FBI study reported that were aware of at least one such error. (All but one of these occurred during proficiency tests or were detected during verification.) Fifty-six out of one hundred sixty-seven responding examiners reported awareness of "an erroneous exclusion (after training)," and ten of these were "on casework: detected after it was reported to contributor." Ulery, Hicklin, Buscaglia, et al., op. cit.

${ }^{410}$ Budowle, Bottrell, Bunch, et al., op. cit.

${ }^{411}$ Koehler, op. cit.

${ }^{412}$ Peterson, Dreyfus, Gische, et al., op. cit.

${ }^{413}$ For an argument against admissibility, see Imwinkelried, E. and D. Kaye. "DNA Typing: Emerging or Neglected Issues." Washington Law Review, 76 (2001): 413.

${ }^{414}$ Williams v. State, 679 A.2d 1106 (Md. 1996).

${ }^{415}$ Ibid., p. 1120.
} 
court specifically rejected "the State's contention that 'It does not matter if fifty percent of their cases were contaminated. What matters is this one." "416 Of course, whatever the result as to the admissibility of errors in other cases, the trial judge has discretion to exclude past errors that are not very probative. Indeed, exclusion would seem to be required if the past errors could not have recurred as a result of corrective action.

Given the emphasis on error rates in post-Daubert opinions, an expert who testifies to an individualization should understand that a false positive error could arise, in theory, for at least two logically distinct reasons. ${ }^{417}$ One is the possibility that somewhere in the world, another individual has a finger that could have produced a latent print with the same distinctive features noted in the latent print in the case. The other is that the examiner erred in identifying the distinctive features or gauging their distinctiveness. A witness can concede that these are logical possibilities — that there are no absolute certainties - without altering an opinion that the defendant is very likely to be the source of a high-quality latent print. Indeed, in presenting the opinion, it might be wise to describe the steps taken to avoid observational and judgmental error. Consequently, the Working Group recommends:

\section{Recommendation 6.3: A testifying expert should be familiar with the literature related to error rates. A testifying expert should be prepared to describe the steps taken in the examination process to reduce the risk of observational and judgmental error. The expert should not state that errors are inherently impossible or that a method inherently has a zero error rate.}

Recommendation 6.3 is not limited to individualization testimony. A witness who testifies to the strength of the evidence without stating that the defendant is the only possible source of the unknown print, as described below in section 6.7, can explain that the evidence is extremely strong, in part because of the protections against erroneous ascertainment of the relevant features, the verification by other examiners, ${ }^{418}$ and the fact that the images are available for verification by still more experts. However, care must be taken in testifying or arguing that a defendant failed to do an independent re-analysis. Suggesting that the defendant has an obligation to verify the findings would be constitutionally objectionable. Nonetheless, if the defendant raises the issue, the prosecution should be permitted to disclose the fact that other experts can confirm (or refute) a reported match. ${ }^{419}$

\footnotetext{
${ }^{416}$ Ibid. See also State v. Proctor, 595 S.E.2d 480 (S. Car. 2004) (regarding proficiency test results as potential impeachment evidence that must be disclosed to the defense under Brady v. Maryland).

417 See Kaye, Bernstein, and Mnookin, op. cit.

${ }^{418}$ Such testimony is routinely received as part of the explanation of the "V" in ACE-V, but its admissibility could be challenged under the hearsay rule and the Confrontation Clause. On the hearsay problem, see Bunche v. State, 5 So.3d 38, 40 n.1 (Fla. Dist. Ct. App. 2009) (collecting conflicting cases).

${ }^{419}$ Imwinkelried and Kaye, op. cit.; National Academy of Sciences, National Research Council, Committee on DNA Forensic Science: An Update. The Evaluation of Forensic DNA Evidence. National Academies Press, 1996, pp. $182-183$.
} 


\subsection{Terminology}

The 2009 NRC report observes that

many terms are used by forensic examiners in reports and in court testimony to describe findings, conclusions, and the degrees of association between evidentiary material (e.g., hairs, fingerprints, fibers) and particular people or objects. Such terms include but are not limited to "match," "consistent with," "identical," "similar in all respects tested," and "cannot be excluded as the source of." 420

According to the committee, the fact that "the forensic science disciplines have not reached agreement or consensus on the precise meaning of any of these terms" is a "critical" problem.

However, clarity would seem more important than uniformity. From the jurors' standpoint, it is not obvious why every testifying expert in every field must use the same phrase to describe a given degree of association. When the expert defines words such as "match" or "identical" in the course of the testimony, the choice of one particular synonym over another should not confuse jurors. What is critical is that the degree of similarity be accurately ascertained and conveyed and that the implications of the observed degree of similarity be described fairly and clearly.

Unfortunately, disagreement exists regarding what terminology is most appropriate and warranted. Therefore, this section describes the traditional forms of testimony about an examiner's findings, along with several possible alternatives that are at least as well founded in our current knowledge of the nature of latent prints and the cognitive capacities of human examiners. The purpose of explicating such alternative formulations is not to insist on the use of any single set of terms but to canvass the range of potentially admissible testimony about the implications of the observed similarities and differences between an unknown print and an exemplar.

\subsubsection{Reporting an Exclusion, Match, or Inconclusive Result}

All of the disciplines that strive to associate a trace with its source involve two logical steps: (1) measuring the similarities and differences between two samples, and (2) assessing the significance of the measurements. ${ }^{421}$ The measurements pertain to properties of the material or mark (e.g., the refractive index of glass, the color of hair, the sizes of DNA fragments, or the concentrations of elements). The significance of finding corresponding characteristics in a pair of samples depends on how often the combination of those characteristics arises when the samples come from the same source as opposed to when they originate from different sources.

A quantity that captures this idea is the likelihood ratio. As previously explained (Chapters 1 and 4), the likelihood ratio states how many times more probable the measurements are when the hypothesis of a common source $(s)$ is true than they are when the hypothesis of a disparate sources $(d)$ is true. When the likelihood ratio is exactly one-when the measured similarities in a

${ }^{420}$ NAS, NRC, Committee on Identifying the Needs of the Forensic Science Community, op. cit., p. 185.

${ }^{421}$ Kaye, D. "Interpretation: A Legal Perspective." In Wiley Encyclopedia of Forensic Sciences, edited by A. Moenssens and A. Jamieson, vol. 3, pp. 1561-65. J. Wiley \& Sons, 2009. 
pair of items is as probable for a common source as a disparate one- the analysis is of no value in informing a decision between these two possibilities. The evidence is logically and legally irrelevant. ${ }^{422}$ On the other hand, when the measurements are far more probable for a common source than for different sources, they are strongly probative of identity $(s)$.

Testimony from examiners who perform pattern matching for identification normally reflects these two steps of measurement and inference. All the phrases quoted in the NRC report"match," "consistent with," "identical," "similar in all respects tested," and "cannot be excluded as the source of"-merely express a finding that the measured features in the two samples being compared show a degree of correspondence. Conversely, when the measurements do not correspond, an examiner may testify to an "exclusion." An exclusion means that the measurements on the two items are so different that they would never (or almost never) arise when the items have a common source, while the measurements could easily be this different when the items come from different sources. Thus, when two fingerprints are so different that they almost certainly could not have come from the same finger, an examiner, via the ultimately subjective process of "evaluation" described in Chapter 3, may conclude that the finger that produced the known print did not produce the questioned print. Finally, a forensic analyst may decline to reach a conclusion about the origin of a trace, such as when an examiner decides that a latent print is not even worth comparing ("not suitable") or that a pair of impressions is worth studying further but that they have too few clear similarities or dissimilarities to warrant a reasonable judgment of inclusion or exclusion ("inconclusive").

\subsubsection{Describing the Significance of a Match: Global Individualization}

The second aspect of pattern matching - describing the significance of the observed degree of matching - has proven contentious in all fields of forensic identification. ${ }^{423}$ For this reason, it is important to consider a spectrum of possible modes of presentation, ranging from the very powerful assertion that every finger leaves marks that are distinguishable from those of every other finger (when the marks are of sufficient quality and extent) to the very weak claim that an observed degree of similarity is at least slightly more likely if the items being compared originated from the same source than if they came from different sources.

The traditional form of testimony in the field of latent print identification is source attribution based on the theory of universal individualization (Chapter 1). One survey of the field explains that

in the friction ridge discipline, an individualization is often reported as, "One latent fingerprint detected on a demand note has been identified as a fingerprint of JOHN DOE." Similarly, when testifying, an examiner often describes the individualization conclusion with a statement such as, "The latent print on Government's Exhibit 10, a revolver, and the fingerprint recorded in the right index finger block on the fingerprint card bearing the name 'John Doe' originated from the same source." ${ }^{24}$

\footnotetext{
${ }^{422}$ McCormick, op. cit., $\S 185$.

${ }^{423}$ Giannelli and Imwinkelried, op. cit.; Faigman, Kaye, Saks, et al., op. cit.; Kaye, Bernstein, and Mnookin, op. cit.

${ }^{424}$ Peterson, Dreyfus, Gische, et al., op. cit. See also Cole, S. "Where the Rubber Meets the Road: Thinking About Expert Evidence as Expert Testimony.” Villanova Law Review, 52 (2007): 803.
} 
Some witnesses combine statements of individualization with expressions of absolute certainty, as in United States v. Hugh, ${ }^{425}$ where "the government's fingerprint expert ... testified that he was ' 100 percent, without a doubt' certain that 'the latent print is that of Nolan Hugh.'"

Such testimony has the virtue of stating clearly and unequivocally the examiner's opinion of what the observed degree of similarity establishes: that the suspect's finger is the source of the latent print. Opinions of "universal individualization" like this one rest on a theory of "universal general uniqueness." 426 In a Massachusetts case, for example, the expert "stated that the prints had been 'individualized' to a Samantha Rivera, and defined the word 'individualized' as meaning 'to the exclusion of all others." 427 This is the classical understanding of "individualization" in forensic science, although, as noted in Chapters 1 and 3, there has been some movement toward using the term to mean any definitive identification of a single individual as the source of a latent print.

Until published studies address the criticisms of universal individualization outlined in Chapter 1, challenges under Rule 702 to the admissibility of absolute statements of individualization will continue. Except for a handful of unreported decisions, ${ }^{428}$ challenges to the admissibility of individualization under the existing ACE-V process have failed, ${ }^{429}$ but the NRC report lends new fuel to attacks on universal individualization testimony. In addition to characterizing ACE-V as un-validated and of doubtful reliability, ${ }^{430}$ the report endorses the view that "in order to pass scrutiny under Daubert, fingerprint experts should exhibit a greater degree of epistemological humility. Claims of 'absolute' and 'positive' identification should be replaced by more modest claims about the meaning and significance of a "match." 431 To consider how this could be accomplished, the next several sections consider some alternatives to the traditional individualization testimony that was based on the theory of global general uniqueness.

\subsubsection{Describing the Significance of a Match: Specific Individualization}

In a particular case, same-source testimony might be justified even if the claim of universal general uniqueness were rejected. Imagine a particular case in which only two individuals could have left the latent print, and an exemplar for one of these two individuals (but not the other) is

\footnotetext{
${ }^{425}$ United States v. Hugh, No. Crim.A. 03-829, 2009 WL 212420 (E.D. Pa. Jan. 28 2009).

${ }^{426}$ Kaye, D. 'Identification, Individualization and Uniqueness: What's the Difference?" Law, Probability \& Risk, 8 (2009): 85.

427 Commonwealth v. Gambora, 933 N.E.2d 50, 56 (Mass. 2010).

${ }^{428}$ See Page, M., J. Taylor, and M. Blenkin. "Forensic Identification Science Evidence Since Daubert: Part IIJudicial Reasoning in Decisions to Exclude Forensic Identification Evidence on Grounds of Reliability." Journal of Forensic Sciences, 56 (2011): 913 - 917. The best known opinions come from a state trial judge in Maryland and from federal trial judges in Pennsylvania and Utah. State v. Rose, No. K06-0545 (Md. Cir. Ct. Oct. 19, 2007); United States v. Llera Plaza, Crim. No. 98-362-10, 11, 12, 2002 U.S. Dist. LEXIS 344 (E.D. Pa. Jan. 7, 2002), vacated, 188 F. Supp. 2d 549 (E.D. Pa. 2002); United States v. Zajac, No. 2:06-cr-00811 CW (D. Utah Sept. 16, 2010).

${ }^{429}$ No reported appellate court opinion has limited testimony of individualization. See U.S. Department of Justice, Office of the Inspector General. A Review of the FBI's Progress in Responding to the Recommendations in the Office of the Inspector General Report on the Fingerprint Misidentification in the Brandon Mayfield Case. U.S. Department of Justice, 2011, p. 20, note 22 (listing federal circuit court opinions).

${ }^{430}$ NAS, NRC, Committee on Identifying the Needs of the Forensic Science Community, op. cit., p. 142.

${ }^{431}$ Ibid. (quoting Mnookin).
} 
available. Furthermore, there is an excellent match between the latent and the exemplar, and the probability of this degree of matching in a finger from a randomly selected individual is a mere one in one trillion. Then the chance that the other (untested) individual also matches is one in one trillion. Now, if the potential suspect population were even slightly larger, say, three individuals, then the chance of a match to either the second or the third untested individual also would be larger — about two in one trillion. If the suspect population were larger still—say, one thousand people - then the chance of a match to at least one of these one thousand people would be roughly one thousand out of one trillion (i.e., one in one billion). In all these small populations, the chance of a duplicate fingerprint is so small that one can be confident that no other match would be found.

The FBI uses such reasoning to make source attributions for DNA evidence when the estimated random-match probability for the specific DNA profile is very small relative to the suspect population. ${ }^{432}$ Courts have upheld these source attributions despite defense arguments that untested relatives might match or that the laboratory could have erred. ${ }^{433}$ The assertion is not that every identifying DNA profile is unique in all populations, but only that the probability of finding no other matching profiles in a smaller population (such as that of the United States) is close to one. For a one-in-one-trillion probability of a random match to a particular DNA profile, for example, the probability that no unrelated resident of the United States would match that profile is approximately $1-\left(3 \times 10^{8}\right) \times 10^{-12}=0.997$.

This reasoning differs from the theory of global individualization of fingerprints in two respects. First, the DNA computation pertains only to the population of the United States and not to the much larger population of the entire world. Second, the DNA computation does not purport to show that every DNA profile in the smaller population is unique to an individual (and any identical twin of that individual). Rather, the computation merely gives the probability that the one profile that has been observed to match the suspect is duplicated in a population of unrelated individuals. The latter difference is often analogized to the famous Birthday Paradox. You can be pretty sure that if you are in a room with 22 other people, your birthday is unique to you. If every birthday is equally frequent in the population and if leap years are ignored, the probability is [1 $(1 / 365)]^{23}=0.94$. But it is more probable than not $(p=0.5073)$ that at least one pair of people in the room has the same birthday. A specific birthday probably is unique in the room, but birthdates are probably not a unique identifier, even in this small population. ${ }^{434}$

For single-source testimony to be admissible under the theory that the random-match probability is so small that the duplication probability in a particular region is negligible, the expert would need to have a reasonable estimate of the random-match probability, or at least an upper bound on this quantity. Statistical models of fingerprint features date back to Galton, but the early models suffer from reliance on untested assumptions of independence and the failure to take into

\footnotetext{
${ }^{432}$ Budowle, B., R. Chakraborty, G. Carmody, et al. "Source Attribution of a Forensic DNA Profile." Forensic Science Communications, 2, no. 3 (2000); Kaye, D. and G. Sensabaugh. "Reference Guide on DNA Evidence." Reference Manual on Scientific Evidence. 3rd ed. National Academies Press, 2000.

${ }^{433}$ United States v. Davis, 602 F.Supp.2d 658 (D.Md. 2009); People v. Cua, 119 Cal.Rptr.3d 391 (Cal. Ct. App. 2011); Young v. State, 879 A.2d 44 (Md. 2005).

${ }^{434}$ For discussion in the context of DNA evidence, see NAS, NRC, Committee on DNA Forensic Science: An Update, op. cit.
}

131 Latent Print Examination and Human Factors: Improving the Practice through a Systems Approach The Report of the Expert Working Group on Human Factors in Latent Print Analysis 
account the spatial relationships of features, distortion, and variability among examiners. ${ }^{435}$ Recent work is intended to address these limitations, and it is possible that a basis for opinions of the individuality for some latent prints in various populations can be established. ${ }^{436}$

\subsubsection{Describing the Significance of a Match: Random-Match Probabilities}

Although specific-individualization testimony has been held admissible for DNA typing, the usual testimony is that there is a high degree of similarity and a low probability of such similarity when the defendant is not the source (but coincidentally possesses similar physical features). When good estimates of the frequency of the feature set exist, a numerical probability statement is admissible in court. ${ }^{437}$

The statistical models for DNA frequencies, being based upon theories of population genetics and data from many samples, are not of much help with more complex patterns such as fingerprints that result from different random processes. However, data-driven statistical analyses of feature combinations could provide highly conservative estimates that should be admissible. When such "improbability testimony" was presented in the past, however, it rested on an inadequate scientific foundation. For example, in Commonwealth v. Drayton, ${ }^{438}$

a fingerprint expert, duly qualified, testified that fingerprints found on the wooden box and prints taken from the defendant showed twelve "points of similarity," and stated his opinion that the fingerprints on the box were those of the defendant. On redirect examination, the prosecutor asked the witness to give his opinion on the statistical probability that prints with twelve points of similarity could be made by two different people. The witness replied that "there is a figure of one out of 387 trillion." 439

On appeal from the resulting conviction, the Supreme Judicial Court of Massachusetts held that although the probability should have been excluded because it "lacked foundation and exceeded the witness's expertise," 440 the "brief, cumulative statement concerning statistics was harmless." ${ }^{441}$

Modern databases and computer technology are capable of providing more defensible probability estimates. One prominent researcher noted that

\footnotetext{
${ }^{435}$ See Pankanti, S., S. Prabhakar, and A. Jain. "On the Individuality of Fingerprints." IEEE Transactions on Pattern Analysis and Machine Intelligence, 24, no. 8 (2002): 1010; Stoney, 2001, op. cit. Claims of global uniqueness founded on older estimates of random-match probabilities are therefore suspect. Yet, such claims persist in textbooks. E.g., Houck, M. and J. Siegel. Fundamentals of Forensic Science. 2nd ed. Academic Press, 2010, pp. 484 $-485$.

${ }^{436}$ See Chapter 4; Kaye, D. "Probability, Individualization, and Uniqueness in Forensic Science Evidence: Listening to the Academies.” Brooklyn Law Review, 75 (Summer 2010): 1163.

437 The development of this legal doctrine is traced in Kaye, The Double Helix and the Law of Evidence, 2010, op. cit.

${ }^{438}$ Commonwealth v. Drayton, 434 N.E.2d 997 (Mass. 1982).

${ }^{439}$ Ibid., p. 1005.

${ }^{440}$ Ibid.

${ }^{441}$ Ibid., p. 1006. Another case that involved a dubious probability calculation is Hicks v. Schurr, 871 F.2d 255 (8th Cir. 1982).
} 
systematic research on the selectivity of fingerprint features [points] towards an extreme selectivity of these features, even when partial and limited information is considered. ... For example ... even very limited configurations of fingerprint minutiae can provide very powerful evidence with match probabilities in the order of 1 in a billion, even without considering the statistical contribution of level 1 features (general pattern, ridge counts, etc.) or other fingerprint features if available. ${ }^{442}$

In the words of one forensic science textbook, "no standard model for random-match probabilities has been adopted for forensic fingerprint casework, but that day is coming."

\subsubsection{Describing the Significance of a Match: Strength of Evidence}

In the types of testimony considered so far, the expert expresses a categorical opinion about who is the source of a latent print or the probability of a match if an individual other than the matching suspect is the source. A substantial body of literature urges forensic scientists to eschew both of these approaches and to confine their testimony to statements about how probable the evidence is when the defendant, as opposed to another (perhaps randomly selected) individual, is the source - that is, to presenting likelihoods or likelihood ratios. ${ }^{444}$

In this likelihood approach, the forensic scientist or analyst never gives an opinion about the prosecution's claim that the defendant is the source. Rather, the expert describes in words or numbers the chances of seeing the evidence in two situations: (1) when the defendant is the source and (2) when someone else is. As noted earlier, the ratio of these quantities, the likelihood ratio, measures the strength of the evidence in favor of identity. The NRC report implicitly endorses this mode of reporting when it states that

although some disciplines have developed vocabulary and scales to be used in reporting results, they have not become standard practice. This imprecision in vocabulary stems in part from the paucity of research in forensic science and the corresponding limitations in interpreting the results of forensic analyses. Publications such as Evett et al., Aitken and Taroni, and Evett provide the essential building blocks for the proper assessment and communication of forensic findings. ${ }^{445}$

Evett and other forensic scientists propose the following table for "reporting the value of the support of the evidence."

\footnotetext{
${ }^{442}$ Champod, op. cit., p. 113.

${ }^{443}$ Houck and Siegel, op. cit., p. 485.

${ }^{444}$ E.g., Aitken, C. and F. Taroni. Statistics and the Evaluation of Evidence for Forensic Scientists. 2nd ed. John Wiley \& Sons, 2004, pp. 414 - 419; Buckleton, J., C. Triggs, and C. Champod. “An Extended Likelihood Ratio Framework for Interpreting Evidence." Science \& Justice, 46 (2006): 69; Robertson, B. and G. Vignaux. Interpreting Evidence: Evaluating Forensic Science in the Courtroom. John Wiley \& Sons, 1995.

${ }^{445}$ NAS, NRC, Committee on Identifying the Needs of the Forensic Science Community, op. cit., p. 186.
} 


\begin{tabular}{|l|l|}
\hline \multicolumn{1}{|c|}{ Range of Likelihood Ratio } & \multicolumn{1}{c|}{ Strength of Evidence } \\
\hline $1<\mathrm{LR} \leq 10$ & Limited evidence to support \\
\hline $10<\mathrm{LR} \leq 100$ & Moderate evidence to support \\
\hline $100<\mathrm{LR} \leq 1000$ & Moderately strong evidence to support \\
\hline $1000<\mathrm{LR} \leq 10000$ & Strong evidence to support \\
\hline $10000<\mathrm{LR}$ & Very strong evidence to support \\
\hline
\end{tabular}

Table 6.1: Strength of likelihood ratios in support of evidence ent $^{446}$

Decades ago, a similar mapping, ranging from "not useful" $(\mathrm{LR}<4)$ to "practically proved" (LR $\geq 499$ ), became the norm in the parentage testing community. ${ }^{447}$

Modern proponents of likelihood-based presentations argue that "the fingerprint profession has to recognize that conclusions of identification to the exclusion of all others are essentially outside the realm of the expert witness. ... Testimony should limit itself to expressing the contribution of the findings in favor of one proposition (identity of sources) versus another (nonidentity of sources). ${ }^{.48}$ In Europe, the Association of Forensic Science Providers has described how practitioners can implement this approach to expert evaluations of evidence. ${ }^{449}$ Even when precise values of the likelihood ratio for a type of evidence is not available, an expert can rely on subjective probabilities in a table, such as the one above, to describe the weight of the evidence without taking the further step of drawing a conclusion about what the evidence proves. In a fingerprint case, an examiner using this framework might testify that the degree of similarity (a "match") is "very strong" evidence that the latent print originated from the defendant's finger 450 because, based on what is known in the field, it is far more probable that this degree of similarity would occur when comparing the latent print with the defendant's fingers than with someone else's fingers. This is certainly a more modest claim than an absolute source attribution based on the theory of global general uniqueness. Furthermore, in the case of a competent and careful comparison, it would be far less vulnerable to the charge of over-claiming. Judicial acceptance of such testimony need not await the admission of likelihood ratios from the automated systems described in Chapter 4.

\footnotetext{
${ }^{446}$ Aitken and Taroni, op. cit.; Evett, I., G. Jackson, J. Lambert, et al. "The Impact of the Principles of Evidence Interpretation on the Structure and Content of Statements." Science \& Justice, 40 (2000): 233. See also Cook, R., I. Evett, G. Jackson, et al. “A Model for Case Assessment and Interpretation.” Science \& Justice, 38 (1998): 151. This table is reminiscent of Jeffrey's scale for interpreting a Bayes factor. Jefferys, H. Theory of Probability, $3 \mathrm{~d}$ ed. Oxford University Press, 1961.

${ }^{447}$ See Hummel, K. and J. Gerchow, eds. Biomathematical Evidence of Paternity. 1982. Joint AMA-ABA Guidelines: Present Status of Serologic Testing in Problems of Disputed Parentage. Family Law Quarterly. 10 (1976): 247.

${ }^{448}$ Champod, C. "Friction Ridge Examination (Fingerprints): Interpretation of." In Wiley Encyclopedia of Forensic Sciences edited by A. Moenssens and A. Jamieson, vol. 3, pp. 1508-11. John Wiley \& Sons, 2009.

${ }^{449}$ Association of Forensic Science Providers. "Standards for the Formulation of Evaluative Forensic Science Expert Opinion." Science \& Justice, 49, no.3 (2009): 161 - 64.

${ }^{450}$ Obviously, other phrases are defensible. The efforts to construct verbal scales are all somewhat arbitrary, both regarding the end points of the intervals and the choice of the words to describe them. Aickin, M. and D. Kaye. "Some Mathematical and Legal Considerations in Using Serologic Tests to Prove Paternity." Inclusion Probabilities
} 
Nevertheless, the strength-of-evidence approach is easier to use when more objective estimates of likelihoods can be computed, and statistical models to estimate likelihood ratios for fingerprint evidence have been developed. They have the potential to replace rigid, categorical opinions with more finely graded, numerical expressions of the probative value of the evidence (see Chapter 4). Some experts favor directly presenting the numerical value of the likelihood ratio to the jury, as is done in some DNA cases. ${ }^{451}$ Others have proposed comparing the likelihood ratio derived from the latent and exemplar in the case at hand to simulated ratios for pairings of the latent print with unrelated exemplars. ${ }^{452}$ The strength of the evidence then can be stated as the proportion of known cases with a lower likelihood ratio than that for the case at hand. For instance, the prosecution could point out that the likelihood ratio is higher than it is for $75 \%$ of cases in which two prints came from the same finger; the defense could reply that the same ratio was lower than it is for $25 \%$ of cases in which the prints came from the same finger. ${ }^{453}$

Of course, for numerical likelihood ratios to be admissible as evidence, the models must be adequately validated and accepted in the scientific community, and the courts must be persuaded that expressing the strength of evidence in the form of a likelihood ratio is not too confusing for juries. ${ }^{454}$ The same concerns were raised with the presentation of likelihood ratios in DNA cases, and this form of testimony has been admitted in that area. ${ }^{455}$

\subsubsection{Describing the Significance of a Match: Posterior Probabilities}

One argument for using the likelihood ratio to grade the strength of the evidence comes from a basic formula of probability theory known as Bayes's rule (see Chapter 1). The formula describes the impact of an item of evidence on the odds that a proposition is true. It states that the odds in favor of the proposition are its prior odds adjusted (multiplicatively) by the likelihood ratio: posterior odds $=$ likelihood ratio $\times$ prior odds. For example, if, before considering a fingerprint match to a suspect, it is assumed that the odds that the suspect left the latent print are 1 to 300 million (roughly the size of the U.S. population), then, for a match with a likelihood ratio of 1 billion, the posterior odds that the suspect is the source is $10^{9} \times 1 /\left(3 \times 10^{6}\right)=333: 1$.

\footnotetext{
${ }^{451}$ See Kaye, The Double Helix and the Law of Evidence, 2010, op. cit.

${ }^{452}$ Gill, P., J. Curran, C. Neumann, et al. "Interpretation of Complex DNA Profiles Using Empirical Models and a Method to Measure Their Robustness.” Forensic Science International, 2 (2008): 9.

${ }^{453}$ See, e.g., Morrison, G. "Measuring the Validity and Reliability of Forensic Likelihood-Ratio Systems." Science \& Justice, 51 (2011): 91 - 98. Some researchers have suggested that Tippett plots could be used to compute such proportions. Such plots express the cumulative distribution function of the log-likelihood ratio. Tippett, C., V. Emerson, F. Lawton, et al. "The Evidential Value of the Comparison of Paint Flakes from Sources Other Than Vehicles." Journal of the Forensic Science Society, 8 (1968): 6. On the use of such plots in other forensic disciplines, see Evett, I. and J. Buckleton. "Statistical Analysis of STR Data." Advances in Forensic Haemogenetics, 6 (1996): 79; Srihari, S., C. Huang, and H. Srinivasan. "On the Discriminability of the Handwriting of Twins." Journal of Forensic Science, 53 (2008): 430. For an example of Tippett plots for fingerprint analysis, see Egli, N., C. Champod, and P. Margot. "Evidence Evaluation in Fingerprint Comparison and Automated Fingerprint Identification Systems-Modelling Within Finger Variability.” Forensic Science International, 167 (2007): 189-95.

${ }^{454}$ There have been various studies of the manner of communicating evidence in DNA cases. E.g., Nance, D. and S. Morris. "Juror Understanding of DNA Evidence: An Empirical Assessment of Presentation Formats for Trace Evidence with a Relatively Small Random-Match Probability.” Journal of Legal Studies, 34 (2005): 395. However, no study has included the use of likelihoods. Kaye, D., V. Hans, B. Dann, et al. "Statistics in the Jury Box: How Jurors Respond to Mitochondrial DNA Probabilities.” Journal of Empirical Legal Studies, 4 (2007): 797.

${ }^{455}$ Kaye, The Double Helix and the Law of Evidence, 2010, op. cit.; cases cited Kaye, Bernstein, and Mnookin, op. cit.
}

135 Latent Print Examination and Human Factors: Improving the Practice through a Systems Approach The Report of the Expert Working Group on Human Factors in Latent Print Analysis 
Computations of this type have been introduced into court, primarily in parentage testing cases arbitrarily using prior odds of one-to-one. In other areas, courts in America and England have been less accommodating. ${ }^{456}$ There are obvious problems in defining the prior probability distribution. Assuming that everyone in the U.S. could have committed a particular crime, as in the example above, is plainly unrealistic.

\subsubsection{Describing the Significance of a Match: No Inference}

When the distinguishing features of trace evidence are extremely rare, the evidence is highly probative - a particular set of features is many times more probable when the unknown trace sample and the known sample have a common source than when they do not. But for most identification technologies, scientific research to quantify the rarity of the set of identifying features is not feasible or available. Examples include hair morphology, compositional analysis of bullet lead, handwriting, and toolmarks. Analysis of these characteristics is surely informative, but it is difficult to provide a sharp estimate of probative value because of the absence of a typology of features that lends itself to estimates of population frequencies and the inability to compute a likelihood ratio from public data. In this situation, analysts in some fields do not even venture an opinion about the significance of the similarities they detect. Thus, in microscopic hair comparisons, unadorned "consistent with" testimony is the norm. In State v. McGrew, ${ }^{457}$ for example, the hair examiner testified as follows:

Court: In regard to the examination. It is simply a physical, visual examination of the hair.

Analyst: Yes sir.

Court: You simply say that one hair looks like another one or it doesn't look like another one.

Analyst: I say it's sufficiently similar to have come from that person or it is dissimilar.

Court: And if you say that it ... [is] similar to come from that person ... that doesn't mean that it comes from that person.

Analyst: It just simply means that it could have come from that person.

Court: And you do not know the statistical percentages of how many people would have similar hair?

Analyst: There are no statistics. It's hard to say. ${ }^{458}$

Similar testimony appears in some cases involving tests for the concentration of elements in bullet fragments and bullets found in a defendant's possession. ${ }^{459}$

Some courts have followed this no-inference approach in cases involving the comparisons of documents and toolmarks. ${ }^{460}$ In United States v. McVeigh ${ }^{461}$ and United States v. Nichols ${ }^{462}$ the

\footnotetext{
456 The case law is analyzed in Kaye, Bernstein, and Mnookin, op. cit., $\S$ 12.8.5.

${ }^{457}$ State v. McGrew, 682 N.E.2d 1289 (Ind. 1997).

${ }^{458}$ Ibid., p. 1291.

${ }^{459}$ Cases are cited in National Academy of Sciences, National Research Council, Committee on Scientific Assessment of Bullet Lead Elemental Composition Comparison. Forensic Analysis: Weighing Bullet Lead Evidence. National Academies Press, 2004, pp. 91 - 94.
} 
government sought to show that a padlock at a quarry from which explosives were stolen had been opened by drilling with a drill bit found in Nichols's home. In the former case, an FBI toolmark examiner testified that "this drill bit produced marks that were contained in the padlock." In the latter case, when the same witness testified that he had attempted to "determine whether or not that drill bit made the impressions contained on that lock cylinder," the district court struck the testimony "because what is permitted here is to show [only] what he saw through the microscope and the comparison microscope and then with his experience and training the similarities that he pointed out." Thus, the toolmark examiner was allowed to testify to the marks, but no more than that. Similarly, a few courts confined testimony to comparing features in handwriting cases and, for a brief moment, in a fingerprint case. ${ }^{463}$

A slight enhancement to pure "consistent with" testimony is a statement that "the probability of a match is greater if the bullets came from the same CIVL [compositionally indistinguishable volume of lead] than if they came from different CIVLs, and the odds that the bullets came from the same CIVL are greater with the matching evidence than without it." 464 In other words, the expert can report that the likelihood ratio is greater than one, but not how much greater.

Although the forensic examiners' experience suggests that the failure to exclude a suspect based upon the evidence is relevant, the jury has no experience of its own with which to judge how probative the findings are. Hair and toolmarks, for example, do differ from one instance to another, ${ }^{465}$ but it is difficult for the jury to know what to make of testimony that the defendant (or the material in the defendant's possession) is consistent with the evidence collected at the crime scene. ${ }^{466}$ Nonetheless, compared to complete exclusion of the match, this truncated presentation makes sense when it is known that the similar characteristics show substantial variation in the population and when an untutored jury is not likely to overestimate the variability. ${ }^{467}$ Thus, one court recently limited a latent print examiner to explaining the basis for a conclusion that "the latent fingerprint ... is consistent with the known print" by describing "the specific characteristics and markers in the prints" and adding that "based on his experience, certain markers are more common or less common." 468

The details of fingerprints are extremely variable, ${ }^{469}$ but those who question the premises of universal individualization would still object that even with no-inference testimony, the jury will overvalue the finding of a match. At the other pole, those who believe that a latent print can

${ }^{460}$ Kaye, Bernstein, and Mnookin, op. cit.

${ }^{461}$ No. 96-CR-68 (D. Colo. May 5, 1997) (transcript).

${ }^{462}$ No. 96-CR-68 (D. Colo. Nov. 10, 1997) (transcript).

${ }^{463}$ See, e.g., Mnookin, J. "The Courts, the NAS, and the Future of Forensic Science.” Brooklyn Law Review, 75, no. 4 (2010): 1209.

${ }^{464}$ NAS, NRC, Committee on Scientific Assessment of Bullet Lead Elemental Composition Comparison, op. cit., p. 97.

${ }^{465}$ Vanderkolk, J. Forensic Comparative Science: Qualitative Quantitative Source Determination of Unique Impressions, Images, and Objects. Academic Press, 2009.

${ }^{466}$ Kaye, Bernstein, and Mnookin, op. cit.

${ }^{467}$ Kaye, D. "The NRC Bullet-Lead Report: Should Science Committees Make Legal Findings?” Jurimetrics Journal of Law, Science, and Technology, 46 (2005): 91.

${ }^{468}$ United States v. Zajac, No. 2:06-cr-00811 CW (D. Utah Sept. 16, 2010).

${ }^{469}$ E.g., Stoney, 2001, op. cit., p. 327. 
match only a single individual in the history of life on Earth would object that the absence of testimony to that effect deprives the jury of useful information.

In sum, the existing practice of latent print experts is to provide a firm opinion regarding whether a named individual is the source of a questioned print (either a universal individualization or an exclusion) or to give no opinion (by stating that the unknown impression is insufficient for a definitive comparison or that the comparison is inconclusive). But other ways to describe the possible association include statements about the strength of the evidence (the likelihoods) or the posterior probability. With appropriate data and validated modeling, such statements could be quantitative (see Chapter 4), but less precise qualitative descriptions of the strength of the evidence or the source probability also are possible. If expert knowledge permits opinions that a latent print came from a single individual and could not have come from anyone else in the world then, a fortiori, it supports opinions such as the following: it is unlikely that the print came from anyone else in a particular locale, it is much more likely to observe such similar prints from the same source than from different people, and the similarities in the prints support the prosecution's hypothesis that the defendant is the source of the questioned print much more than they support the defendant's hypothesis that they originated from different fingers. ${ }^{470}$ Given the current state of scientific and professional knowledge, however, it is best to avoid testimony based on the theory of global general uniqueness. Recommendation 3.7, that examiners not testify to an identification to the exclusion of all other individuals in the world, requires other, more conservative methods for conveying the probative value of a match. For that reason, this chapter identifies a broad spectrum of alternatives. The Working Group did not reach a consensus on which of these alternatives to universal-individualization testimony is best.

\subsection{Internal Review of Reports and Testimony}

Chapters 4 and 5 emphasize the role of forensic scientists and examiners in communicating their findings to a variety of audiences, including criminal investigators, lawyers, and judges and juries. The recommendations include practices to facilitate the accurate and fair transmission of forensic investigations to these groups. The final recommendation is that a system be instituted to monitor the reports and testimony of practitioners. Performance reviews can ensure that the latent print unit's policies for reporting and testifying are followed and can provide feedback to sustain and improve performance of these tasks.

\footnotetext{
${ }^{470}$ Even the current terminology of identification, exclusion, or inconclusive to describe the outcome of the Evaluation phase of ACE-V could be redefined using the concept of relative likelihood. For example, the definition of these terms in the first two sample reports in the Appendix to Chapter 5 could be rephrased as follows:

Evaluation is the formulation of a conclusion based upon the information gathered during analysis and comparison. The evaluation can result in three outcomes: (1) an exclusion is an opinion that the level of disagreement in comparable areas is far more likely to be observed if the two prints did not originate from the same source than if they came from the same finger; (2) an identification is an opinion that the level of agreement in comparable areas is far more likely if the two prints originated from the same finger than if they came from different sources; or (3) an inconclusive result occurs when the information in comparable areas is inadequate for an identification or an exclusion.
} 


\section{Recommendation 6.4: An agency that employs latent print examiners should establish requirements and guidelines for reporting, documentation, and testimony. The agency should review a sample of every examiner's case reports, documentation, and testimony and affidavits at least annually to verify compliance with these requirements and guidelines.}

SWGFAST and the FBI give similar advice, but their recommendations allow a survey form and statements from court officials to substitute for direct observation of live, recorded, or transcribed testimony. ${ }^{471}$ Presumably "court officials" means judges or lawyers, but review by laboratory managers and accrediting bodies as part of periodic inspections or in response to complaints from lawyers or their clients would be more appropriate to identify adherence to professional canons and organizational policies.

\subsection{Summary}

This chapter describes the nature and role of expert testimony, pretrial communications between experts and lawyers, and the legal and ethical principles applicable to expert testimony. It considers the admissibility of different types of testimony about the possibility of erroneous identifications or exclusions. It also presents a spectrum of conceivable modes of conveying expert knowledge and findings about the possible association between a friction skin impression and its source. These range from a bare description of the relevant similarities, to statements about the strength of evidence (likelihoods), to outright conclusions about the source (source attributions or probabilities). Finally, the chapter discusses quality assurance and control for testimony.

\footnotetext{
${ }^{471}$ Scientific Working Group on Friction Ridge Analysis, Study and Technology. Quality Assurance Guidelines for Latent Print Examiners. Guideline 5.5.1, Version 3.0. Scientific Working Group on Friction Ridge Analysis, Study and Technology, September 2006; Peterson, Dreyfus, Gische, et al., op. cit.
} 


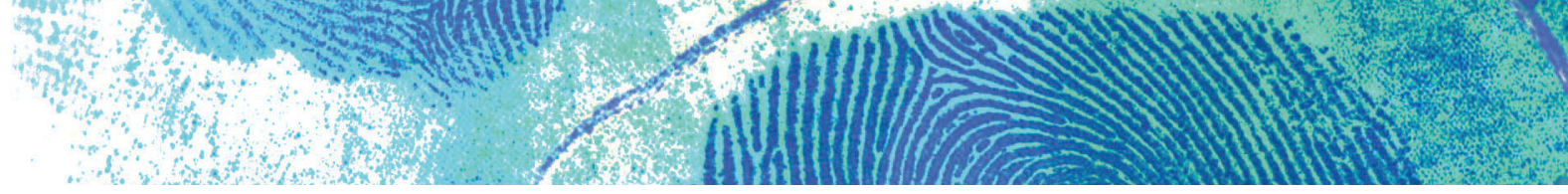

\section{Chapter 7: A Systems Approach to the Work Environment}

\section{Introduction and Scope}

The environment in which latent print examiners work encompasses physiological and cognitive factors; management and leadership culture, communications, and collaboration opportunities; and the physical workspace. Well-designed work environments can improve productivity, increase user satisfaction, and reduce the risk of errors and injuries. Conversely, poorly designed environments contribute to poor performance. When an error occurs, most people tend to blame other humans rather than to consider the design, environmental working conditions, or management culture of the total system. ${ }^{472}$ Yet errors are often the consequence of an entire system. ${ }^{473}$

A study of human factors examines human operators' physical and psychological needs, capabilities, and limitations in both normal and emergency operations. A thorough analysis of workstations, job demands, mental workload, organizational characteristics, training needs, and supervisory systems is necessary to facilitate and enhance performance. Large-scale disasters, such as the accidents at the Three Mile Island and Chernobyl nuclear power plants, the Bhopal pesticide plant, and the Challenger space shuttle dramatically highlight the need for such analyses. ${ }^{474}$

Figure 7.1 sketches an example of a human factors framework that, if not properly understood, can lead to adverse events. This chapter addresses issues displayed in Tiers 1-3 of the figure and analyzes the latent print examiners' work environments to identify factors that could affect their performance. It enumerates the factors that contribute to an effective latent print analysis work environment, and it describes the anatomical, physiological, and psychological aspects of latent print examiners in their environment. It also suggests ways for managers to reduce injuries and errors; to increase productivity, efficiency, and effectiveness; and to improve the comfort of latent print examiners.

\footnotetext{
${ }^{472}$ Luthar, S., D. Cicchetti, B. Becker. "Research on Resilience: Response to Commentaries." Child Development, 71, no. 3 (2003): 573.

${ }^{473}$ Hopp, P., C. Smith, B. Clegg, et al. "Interruption Management: The Use of Attention-Directing Tactile Cues." Human Factors, 47 (2005): 1; National Academy of Sciences, Institute of Medicine, Committee on Quality of Health Care in America. To Err Is Human: Building A Safer Health System. National Academies Press, 1999; Chignell, M. and J. Waterworth. Multimedia, Handbook of Human Factors and Ergonomics. 2nd ed. John Wiley \& Sons, 1997; Bagot, G. "Ergonomics in the Laboratory Environment." Nursing Management, 25 1994): 50; Henning, R., P. Jacques, G. Kissel, et al. "Frequent Short Rest Breaks From Computer Work: Effects on Productivity and Well-Being at Two Field Sites.” Ergonomics, 40 (January 1997): 78; Ezzelle, J., I. Rodriguez-Chavez, J. Darden, et al. "Guidelines on Good Clinical Laboratory Practice: Bridging Operations Between Research and Clinical Research Laboratories." Journal of Pharmaceutical and Biomedical Analysis, 46 (January 2008): 18; Grogan, E., R. Stiles, D. France, et al. "The Impact of Aviation-Based Teamwork Training on the Attitudes of Health-Care Professionals." Journal of the American College of Surgeons, 199 (December 2004): 843.

${ }^{474}$ Shrivastava, P., I. Mitroff, D. Miller, et al. "Understanding Industrial Crises.” Journal of Management Studies, 25, no. 4 (1988): 285.
} 


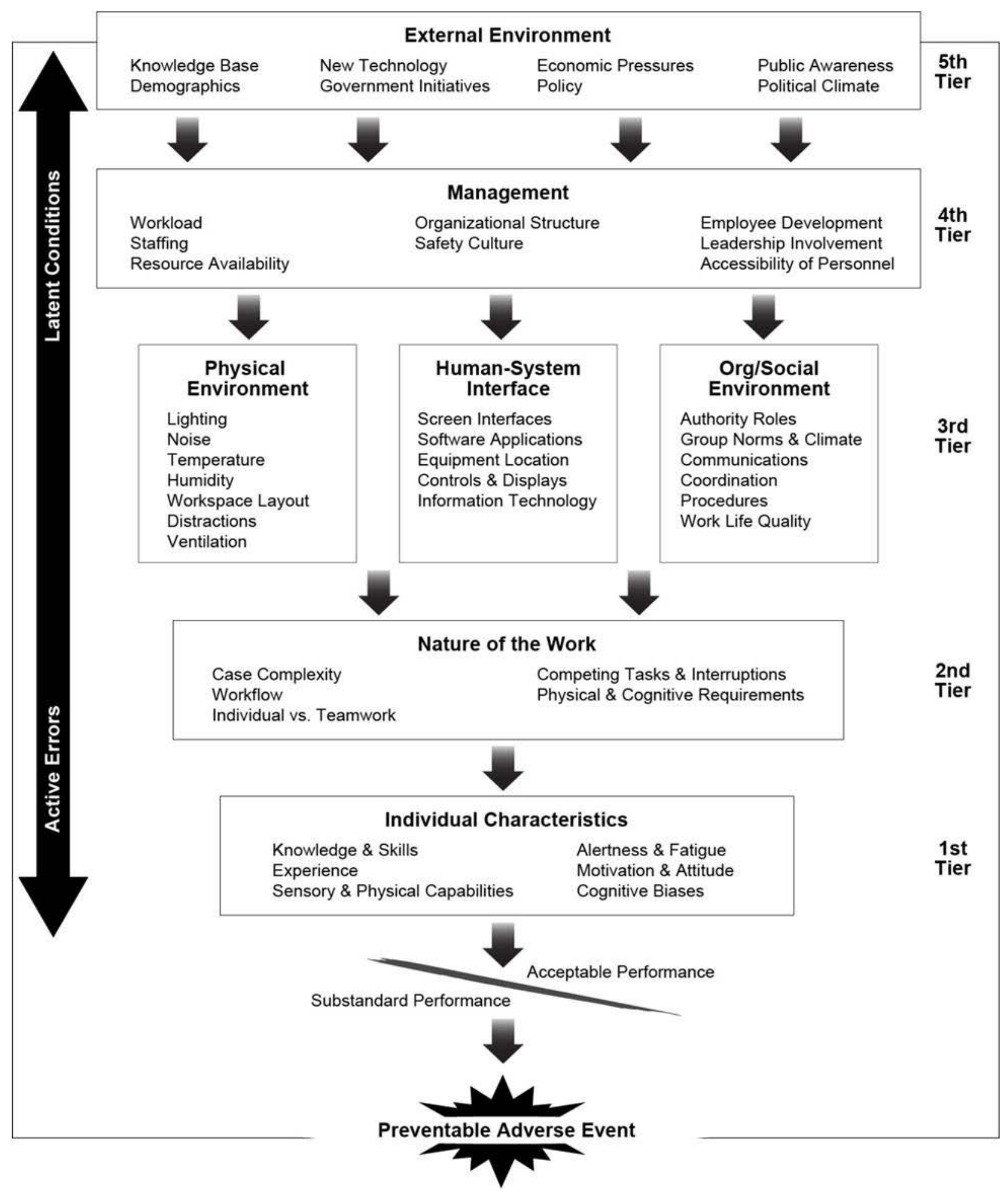

Figure 7.1: A human factors framework ${ }^{475}$

${ }^{475}$ Image adapted from Henriksen, K., E. Dayton, M. Keyes, et al. "Understanding Adverse Events: A Human Factors Framework." In Patient Safety and Quality: An Evidence-Based Handbook for Nurses edited by Ronda G. Hughes. Agency for Healthcare Research and Quality, 2008. 


\subsection{Human Factors and the Work Environment}

The study of human factors focuses on the interaction between human and products, decisions, procedures, workspaces, and the overall environment encountered at work and in daily living. ${ }^{477}$ As the Institute of Medicine explains, most factors that give rise to preventable adverse events are systemic. ${ }^{478}$ These events are not just the result of isolated or idiosyncratic behavior.

Often, people are required to work in poorly designed environments that have not had human strengths and limitations incorporated into the design process. A work environment can be disruptive, stressful, and unsafe, leading to unnecessary fatigue. It is well understood in various industries, including "high-risk industries" such as the medical profession, that a physical work environment purposefully designed for the nature of the work to be performed will result in improved efficiency and productivity. ${ }^{479}$ Proper design of the physical workspace, standardization of facility systems and equipment, ventilation systems for pathogen control, appropriate and adjustable lighting, and noise reduction are necessary for lowering stress and improving performance.

\section{Box 7.1: Three Mile Island Accident}

The Three Mile Island nuclear power plant accident is the most investigated accident in the history of the commercial nuclear industry. Because human factors considerations were not included in the design of the control room, operators experienced problems locating or accessing the tools and information they needed.

- System controls were not located near the instruments that displayed the condition of the system. For example, operators could not view the indicator display for the high-pressure system while operating the throttle valve to adjust pressure.

- Some instruments looked very similar and were located near one another but controlled different functions.

- Other instruments were difficult to read due to glare from poor lighting or obstruction by other controls.

- Throughout the control room, there was no consistent meaning of indicators (such as lights and alarms) or function of instruments (such as levers and knobs) between controls.

- At the time of the accident, operators in the control room heard 3 alarms and saw more than 1,600 blinking lights.

- Operators had not received adequate stress training to enable them to cope with such a large-scale, multiple systems failure. ${ }^{476}$

These design problems illustrate the range of situations and conditions that the human factors approach should consider.

\footnotetext{
${ }^{476}$ Meshkati, N. "Human Factors in Large-Scale Technological Systems' Accidents: Three-Mile Island, Bhopal, Chernobyl." Organization \& Environment, 5, no. 2 (June 1991): 133-54.

${ }^{477}$ Sanders, J. and E. McCormick. Human Factors Engineering and Design. McGraw-Hill Science/Engineering/Math, 1993.

${ }^{478}$ NAS, Institute of Medicine, op. cit.

${ }^{479}$ Ulrich, R., X. Quan, C. Zimring, et al. "The Role of the Physical Environment in the Hospital of the 21st Century: A Once-In-A-Lifetime Opportunity." In Designing the 21st Century Hospital Project, The Center for Health Design. May 2005. See also Chapanis, A., W. Garner, and C. Morgan. Applied Experimental Psychology: Human Factors Engineering Design. Wiley, 1985 ("It is in the system design of the work environment as a whole where efficiencies and productivity will be realized.").
} 
Sound workplace design begins with a thorough understanding of user requirements. Since the early 1970s, architects have used methods, similar to function and task analysis techniques developed by human factors practitioners, that inventory all of the activities that will be performed in an environment to ensure that the appropriate requirements are defined and implemented. ${ }^{480}$

The International Organization of Standardization's standard on ergonomic requirements (ISO 9241) defines usability as "the extent to which a product can be used by specified users to achieve specified goals with effectiveness, efficiency, and satisfaction in a specified context of use." 481

In its narrowest sense, usability involves the evaluation of a system; in its broadest sense, usability involves users throughout the system lifecycle (i.e., through the requirements definition, design, development, and evaluation phases). ${ }^{482}$ The most successful designs involve users iteratively starting with the early stages of design to develop and refine the designs. ${ }^{483}$ This usercentered design process involves:

- Early focus on users, their tasks, and their environments;

- Active involvement of users;

- Appropriate allocation of function between user and system;

- Incorporation of user-derived feedback into the design; and

- Iterative design, where a prototype is designed, tested, and modified..$^{484}$

By contributing to the design of a usable work environment, latent print examiners can enhance ease of use, improve user performance and satisfaction, and reduce system complexity and support and training costs. The goal of designing a work environment following usability principles is to improve performance, to increase accuracy, to decrease time to prepare latent prints for search, to decrease support and training costs, and to increase user acceptance. Appendix A provides additional information on user-centered design.

\section{Recommendation 7.1: A structured approach that involves users throughout the entire design and implementation process should be followed when designing technology systems and the physical work environment. In addition, forensic service providers should perform usability testing to optimize user performance and user satisfaction before deployment.}

\footnotetext{
${ }^{480}$ Zimring, C., M. Rashid, and K. Kampschroer. "Facility Performance Evaluation (FPE).” Whole Building Design Guide. National Institute of Building Sciences. 2010.

${ }^{481}$ International Organization for Standardization. Ergonomic Requirements for Office Work with Visual Display Terminals (VDTs) - Part 11: Guidance on Usability, ISO 9241-11:1998. International Organization for Standardization, 1998.

${ }^{482}$ U.S. Department of Commerce, National Institute of Standards and Technology. Usability \& Biometrics: Ensuring Successful Biometric Systems. National Institute of Standards and Technology, June 11, 2008.

${ }^{483}$ Nielsen, J. Usability Engineering. Morgan Kaufmann, 1993.

${ }^{484}$ Maguire, M. "Context of Use Within Usability Activities.” International Journal of Human-Computer Studies, 55 (2001): 453-83
} 


\subsection{A Human Factors Approach to Latent Print Practices}

A truly usable work environment considers the needs of the latent print examiners throughout the design, development, and evaluation process. This process requires environmental designers to define the context of use (including the users' individual characteristics), to define the user's and the organization's requirements for the space, to develop a design solution to meet those requirements, and to conduct user evaluations to ensure that the environment is meeting all requirements as efficiently as possible. ${ }^{485}$

\subsubsection{Methodology}

A human factors approach focuses on users' needs and expectations and integrates direct user feedback throughout the design process. To achieve this goal, human factors experts may observe or interview users to better understand their needs, work processes, and the methodologies they apply in completing their work.

To better understand latent print examiners' methodologies and processes as well as their environments, the National Institute of Standards and Technology (NIST) Visualization and Usability Group interviewed a total of 16 latent print examiners from the Federal Bureau of Investigation (FBI), Department of Defense, Central Intelligence Agency, U.S. Secret Service, and the New York State Department of Criminal Justice Services. Following these initial interviews, the team spent 2 days observing latent examiner teams at the Maryland State Forensic Laboratory, FBI, and United States Immigrant Status Indicator Technology (US-VISIT) while they performed their usual work tasks in their usual work environments.

After approximately 21 hours of interviews and 32 hours of observation sessions, the team discovered that each organization uses a well-defined process to decide who performs which task, how they complete each task, and how they transition between tasks. The agencies also require specific documentation for each task and utilize procedures for transitioning the documentation through the steps. Each organization and its examiners are very comfortable with their processes and, to some extent, proud of them. These processes are tailored or unique to each organization.

The physical layout of each facility is very different and seems to depend on the size of the organization. Of the surveyed organizations, many have a separate workspace for the chemical processing of the evidence. Most examiners have a small desk, and in the larger organizations, the examiners' desks are in small cubicles. Most of the desks are covered with stacks of paper, including photos and printouts. Printing and scanning are consistently important activities, and many desks include printers and scanners. The impression from all of the interviews is that the organizations are "paper bound." Structured forms seem to be essential to all organizations.

Some organizations use "shops" or task-specific stations. For example, examiners use one workstation exclusively for case management, another for accessing the state automated

\footnotetext{
${ }^{485}$ International Organization for Standardization. Human-Centered Design Process for Interactive Systems, ISO 13407:1999. Withdrawn, 1999. Revised by International Organization for Standardization. Ergonomics of HumanSystem Interaction -Part 210: Human-Centered Design for Interactive Systems, ISO 9241-210:2010. International Organization for Standardization, 2010.
} 
fingerprint identification system (AFIS) database, another for accessing the local AFIS database, and another for linking to the FBI's Integrated AFIS database. The examiner moves the physical evidence and associated documentation from station to station when working through the steps of the process.

In short, there is no one specific or even general solution to the design and specification of the laboratory environment. Design specifications must be tailored to individual departments. However, although each organization has a somewhat different process for examining latent prints, the NIST usability team recognized general similarities.

\section{Common Features of the Manual Examination Process}

Examiners use a manual process when the latent prints are not of AFIS quality or when there is a known suspect and it is necessary to eliminate victim prints. The manual examination process generally includes the following steps:

1. Prepare package (includes lift cards and latent photos);

2. Confirm package contents;

3. Assign card numbers to lift cards and latent photos;

4. Determine suitability of prints;

5. Mark latent print with symbols to document possible anatomical regions and print orientation;

6. Pull exemplar prints for comparison;

7. Begin comparison process on suitable prints;

8. Document results of the comparison; and

9. Conduct verification and technical review.

Common Features of the AFIS Process

When using AFIS, the examiners at the agencies studied generally perform the following steps:

1. Select a latent print to search;

2. Scan the print into the system or obtain a digital print;

3. Process the digital image in external image processing software;

4. Enter case data;

5. Encode unique ridge characteristics;

6. Set search criteria;

7. Launch AFIS search;

8. Examine the resulting candidate images;

9. Conduct verification and technical review; and

10. Document additional case data.

\subsubsection{Work Environment Usability Goals}

Environment designers, including software developers, should apply the user-centered design process to identify the requirements of the organization. Then, they should translate usability goals into measurable objectives for the latent print examiner environment. Environment designers can use these metrics to assess an environment's usability. Examples of commonly used usability criteria include: 
- Success rate (effectiveness): Can users successfully work in the environment?

- Time on task (efficiency): Can users perform their tasks quickly in the environment?

- Time to learn a task (learnability): How long does it take a user to learn a product or the system?

- Memorability: Can users remember how to use the system, and does it aid the user in reducing the potential for error?

- Satisfaction: Are users comfortable in the environment? ${ }^{486}$

The tables and additional information in Appendix A provide a short description of each of these usability goals and include some questions for environment designers to consider.

\subsubsection{Design Approach for Latent Print Examination Environment}

A user-centered design process involves users throughout the product lifecycle. When used for latent print examiners, the approach includes:

- Identifying the types of users who will be using the workspaces (e.g., latent print examiners, forensic specialists, lab managers);

- Defining the context of use, including the operational environment (physical environment), user characteristics (anthropometrics), tasks, and social environment;

- Determining the user and organizational requirements, including business requirements, visual requirements, environmental requirements, and technical requirements;

- Developing the design solution, including the physical space (e.g., lighting, air quality, workspace, and noise), system design, and user interface; and

- Conducting an evaluation, including usability testing of the visual and environmental requirements of latent print examination tasks. ${ }^{487}$

The design process reflects the fact that all aspects of the workflow and environment are interrelated. An examination of a forensic service provider's process map and an independent detailed analysis of how work is actually performed can reveal procedures that can lead to error. Consideration should be given to the nature and demands of the work, the tools and technologies used, the physical environment, and the organizational conditions, such as the level of communication and collaboration among the individuals who perform the work or use the end product. These elements are interdependent; a change in one work element has implications for the other elements.

\subsection{Observations of Impact Factors}

Latent print comparisons require concentration. The environment in which an examiner works influences his or her ability to maintain the necessary level of attentiveness. It is not enough to consider only how the examiner will fit and function in the space from a physical perspective.

\footnotetext{
${ }^{486}$ Nielsen, op. cit.

${ }^{487}$ Maguire, op cit.; ISO 13407:1999 (withdrawn), op. cit.
} 
Attention to the impact that the physical workspace may have on cognitive processes, including attentiveness, awareness, perception, reasoning, and judgment, is critical.

The following factors, which are discussed below, could influence the latent print examiner's work: occupational health and safety; medical surveillance; workstation configurations; software applications and systems; screen interfaces; the placement, type, and format of instructions, assistance, and help documentation; air quality, including temperature and humidity; lighting; noise; interruptions or distractions; location of the workstation; and stress.

\subsubsection{Occupational Health and Safety Issues}

Occupational health and safety within the forensic laboratory system is mandatory. However, workplace safety is not always a conscious or primary concern of latent print examiners. They are generally busy with casework, trial preparation, proficiency testing, and other concerns. The latent print examiner has the potential to come in contact with most types of physical evidence submitted to the forensic service provider. Hazards may originate from the item of evidence through sharp edges, chemical and biological contamination, or other hazards; the evidence may also include firearms, ammunition, or explosives. Of special concern are items contaminated or stained with human bodily fluids, which may contain blood-borne pathogens. Additionally, latent print processing may employ hazardous chemicals, particulates, and intense light sources. Chemical exposure is a serious concern. During the processing of latent prints, examiners could be exposed to fumes, dust, and toxic or caustic chemicals. Additionally, postanalysis protection of certain latent examinations on physical evidence must be employed to eliminate the chance of contamination to persons in the evidence chain of possession. This typically is accomplished by storing the evidence in a container that prevents trace reagent exposure.

An accident can have an effect on an examiner's efficiency and performance quality. For example, a malfunctioning fume hood could expose the examiner to vapors that impair performance. Likewise, an employee who slips and falls in the work area could sprain a muscle or fracture a bone, requiring medical attention and possibly resulting in long-term pain or limited range of motion or dexterity. An injured examiner may have to compensate during the examination procedure, and this could affect the results.

Many standards are relevant here, including Occupational Safety and Health Administration (OSHA) Standards, 29 Code of Federal Regulations (CFR) 1910, 1960, 1904; OSHA's bloodborne pathogens standard (29 CFR 1910.1030); and OSHA's requirements for personal protective equipment (29 CFR, 1910 Subpart I). Guidelines for safety committees are found in 29 CFR 1960. Airborne contaminant limits are described in 29 CFR 1910.1000. 


\subsubsection{Medical Surveillance}

Vision testing is important for latent print examiners. Studies indicate that age, environment, and task-specific activity can cause vision to deteriorate. ${ }^{488}$ It is not clear which tests are the most appropriate for evaluating vision challenges for latent print analysis. ${ }^{489}$ Table 7.1 indicates the American Optometric Association's recommended frequency for eye examinations for adult patients. Individuals at risk "include those with diabetes, hypertension, or a family history of ocular disease (e.g., glaucoma, macular degeneration) ... ; those working in occupations that are highly demanding visually or eye hazardous; those taking prescription or nonprescription drugs with ocular side effects; those wearing contact lenses; and those with other health concerns or conditions. $" 490$

\begin{tabular}{|l|l|l|}
\cline { 2 - 3 } \multicolumn{1}{c|}{} & \multicolumn{2}{c|}{ Examination Interval } \\
\hline \multicolumn{1}{c|}{ Patient Age } & \multicolumn{1}{c|}{ Asymptomatic (Risk Free) } & \multicolumn{1}{c|}{ At Risk } \\
\hline 18 to 40 years & Every 2 years & Every 1 to 2 years or as recommended \\
\hline 41 to 60 years & Every 2 years & Every 1 to 2 years or as recommended \\
\hline 61 years and older & Every 1 year & Every 1 year or as recommended \\
\hline
\end{tabular}

Table 7.1: Recommended eye examination frequency for adult patients ${ }^{491}$

Various tests are available to examine visual performance. For example, the Snellen chart is often used to measure visual acuity. Likewise, the Amsler Grid, a square grid with a black dot in the center, is used to detect changes in the central visual field. Proper use of the grid can detect very subtle changes in vision caused by a small amount of fluid under the retina.

Contrast sensitivity tests check for the ability to differentiate between light and dark. Regular use of the Hamilton Veale contrast sensitivity test, illustrated in Figure 7.2, is one effective way to

\footnotetext{
${ }^{488}$ Sunness, J., G. Rubin, C. Applegate, et al. "Visual Function Abnormalities and Prognosis in Eyes with AgeRelated Geographic Atrophy of the Macula and Good Visual Acuity." Ophthalmology, 104 (1997): 1677.; Sunness, J., G. Rubin, A. Broman, et al. "Low Luminance Visual Dysfunction as a Predictor of Subsequent Visual Acuity Loss from Geographic Atrophy in Age-Related Macular Degeneration.” Ophthalmology, 115 (2008): 1480; Midena, E., A. Degli, M. Blarzino, et al. "Macular Function Impairment in Eyes with Early Age-Related Macular Degeneration.” Investigative Ophthalmology \& Visual Science, 38 (February 1997): 469; Abramov, I. and J. Gordon. "Color Vision Panel Tests: A Metric for Interpreting Numeric Analytic Indices." Optometry \& Vision Science, 86 (2009): 146; Montés-Micó, R. and T. Ferrer-Blasco. "Contrast Sensitivity Loss in the Peripheral Visual Field Following Laser in Situ Keratomileusis.” Journal of Cataract and Refractive Surgery, 33 (2007): 1120; Kon, C. and D. De Alwis. “A New Colour Vision Test for Clinical Use.” Eye (London, England), 10, pt. 1 (1996): 65-74. ${ }^{489}$ Powers, M. "Paper Tools for Assessing Visual Function.” Optometry \& Vision Science, 86 (2009): 613.; Bartlett, H., L. Davies, and F. Eperjesi. "The Macular Mapping Test: A Reliability Study.” BMC Ophthalmology, 10 (2005): 18; Leat, S. and G. Woo. "The Validity of Current Clinical Tests of Contrast Sensitivity and Their Ability to Predict Reading Speed in Low Vision.” Eye (London, England), 11, pt. 6 (1997): 893; Witmer, M., C. Margo, and M. Drucker. "Tilted Optic Disks." Survey of Ophthalmology, 10 (2010): 403; Barnhardt, C., S. Clock, B. Demmer, et al. "Color Vision Screening for Individuals with Intellectual Disabilities: A Comparison Between the Neitz Test of Color Vision and Color Vision Testing Made Easy.” Optometry, 77 (2006): 211; Isaac, D., M. Avila, and A. Cialdini. "Comparison of the Original Amsler Grid with the Preferential Hyperacuity Perimeter for Detecting Choroidal Neovascularization in Age-Related Macular Degeneration.” Optometry, 79 (2008): 397.

${ }^{490}$ American Optometric Association. Comprehensive Adult Eye and Vision Examination. American Optometric Association, 2005.

${ }^{491}$ Adapted from American Optometric Association, op. cit.
} 
monitor contrast sensitivity function over time. ${ }^{492}$ Forensic service providers should perform other vision tests regularly so that examiners know when they need to seek corrections to their sight to maintain high visual acuity.

\section{Recommendation 7.2: Forensic service providers should institute medical surveillance for examiners with respect to activities specific to friction ridge examination. Corrective measures should be taken when appropriate and recorded. The program should include a baseline visual examination followed by annual vision testing to detect vision deficiencies that may affect interpretation of the evidence. The federal government should support research to determine the most appropriate tests of visual function for friction ridge examiners.}

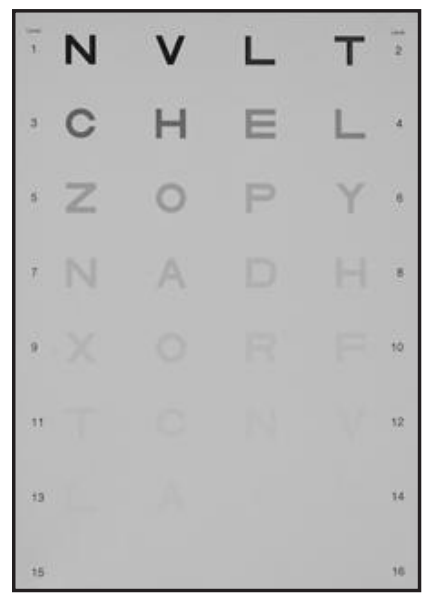

Figure 7.2: The Hamilton Veale contrast sensitivity test

\subsubsection{Workstation Configuration}

Workstation considerations include the physical location and design of the workstation as well as the desk height, counter height, and other spatial layout requirements. Latent print examinations require sufficient space for the latent prints, exemplars, documentation worksheets, magnifying glasses, pointers, writing instruments, and desktop task lighting. The space should permit the required materials to be laid out for the sequential process and to be accessible without undue attention or effort. Clutter is a potential source of frustration and error.

Examiners experience physical problems, such as repetitive motion injuries, from a lack of ergonomic workstation design. Complicating this design issue is the fact that many examiners

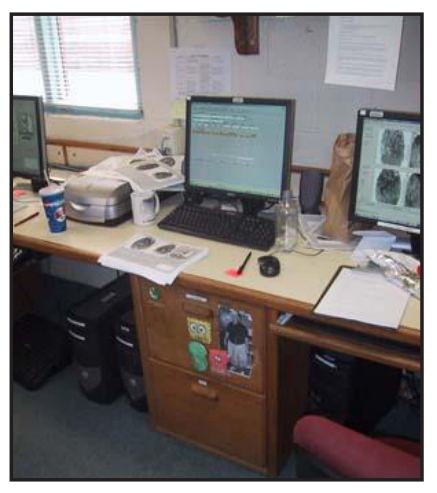

Figure 7.3: An example of a poorly designed workstation are required to share workstations across shifts. Inadequacies can include nonadjustable keyboards; awkward positioning of the mouse; chairs, desks, and monitors that are not adjustable to accommodate for different user heights; inadequate desk space for papers, work products, and equipment; insufficient leg and knee room under the desk; furniture that impedes equipment access; and lighting that cannot be adjusted for using magnifiers.

The working environment and user work posture are critical in preventing repetitive motion injuries. The impact of ergonomics on individual productivity in domains such as automobile manufacture, food processing, and healthcare is well documented. ${ }^{493}$ Good ergonomic designs

\footnotetext{
${ }^{492}$ The Hamilton Veale contrast sensitivity chart should be used in conjunction with an Amsler Grid to detect visual function loss in all cases with macular degeneration.

${ }^{493}$ Cocci, S., K. Namaisvayam, and P. Bordi. "An Investigation of Ergonomic Design and Productivity Improvements in Foodservice Production Tables.” Foodservice Research International, 16 (2005): 53; Kolich, M. and S. Taboun. "Ergonomics Modeling and Evaluation of Automobile Seat Comfort." Ergonomics, 47, no. 8 (June 22, 2004): 841-63; Stone, R. and R. McCloy. "Ergonomics in Medicine and Surgery." BMJ, 328, no. 7448 (May 6, 2004).
} 
increase output per worker and reduce errors, accidents, injuries, illnesses, training time, maintenance time, absenteeism, and turnover. Although no studies have focused on latent print examiners, there is no reason to believe that the results would not generalize from related occupations.

At a minimum, an examiner's workstation should have keyboard shelves that are adjustable to accommodate different heights; computer mice that can be repositioned; chairs that are adjustable for height, arms, and back support; desktops that have mechanisms for adjusting tilt and height; monitors that are adjustable both in height and user proximity; and a desk with sufficient workspace, legroom, and knee space. Applicable standards include ISO 9241302:2008. . $^{494}$

\subsubsection{Software Applications and Systems}

It is critical to include latent print examiners in the design of all solutions, especially technology solutions. Eliciting requirements from latent examiners and having them participate in usability testing is necessary to create successful and usable products and processes. The usability testing and evaluation process permits the user to provide data about what works and what does not. The iterative nature of the process allows changes to produce an optimized software application or solution. Too often, usability evaluations are carried out, if at all, only after a product has been designed, developed, and deployed. Applicable standards are ISO/IEC 9126, ${ }^{495}$ ISO 9241-210, ${ }^{496}$ and ISO/IEC $25062^{497}$ (software engineering, software product quality

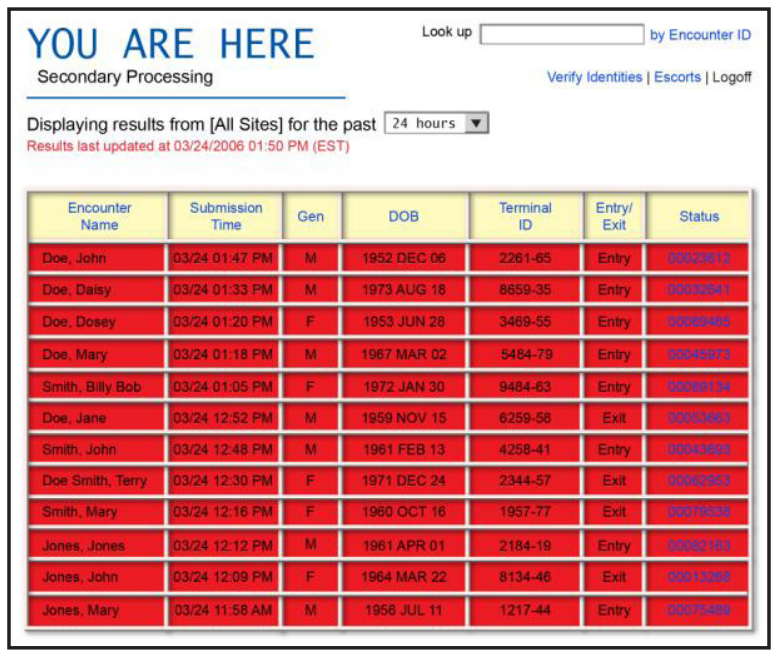

Figure 7.4: An example of a poorly designed user interface requirements and evaluation, and common industry format for usability test reports).

\footnotetext{
494 International Organization for Standardization. Ergonomics of Human-System Interaction-Part 302: Terminology for Electronic Visual Displays, ISO 9241-302:2008. International Organization for Standardization, 2008; International Organization for Standardization. Ergonomics of Human-System Interaction-Part 305: Optical Laboratory Test Methods for Electronic Visual Displays, ISO 9241-305:2008. International Organization for Standardization, 2008. Both of these standards replace the withdrawn International Organization for Standardization. Ergonomic Requirements for Work with Visual Displays Based on Flat Panels), ISO 13406:1999 (withdrawn). International Organization for Standardization, 1999.

${ }^{495}$ International Organization for Standardization. Software Engineering_Product Quality, ISO/IEC 9126:2001. International Organization for Standardization, 2001.

${ }^{496}$ International Organization for Standardization. Ergonomics of Human-System Interaction-Part 210: HumanCentered Design for Interactive Systems, ISO 9241-210:2010. International Organization for Standardization, 2010. Replaces the withdrawn standard ISO 13407:1999, op. cit.

${ }^{497}$ International Organization for Standardization. Software Engineering-Software Product Quality Requirements and Evaluation (SQuaRE)_Common Industry Format (CIF) for Usability Test Reports, ISO/IEC 25062:2006. International Organization for Standardization, 2006.
} 


\subsubsection{Screen Interfaces}

Poorly designed software interfaces were observed in some laboratories. For example, in Figure 7.4, the color combination (i.e., red background with blue text) is difficult to read, causes eyestrain, and has the potential to increase errors. The applicable standards are ISO/IEC $9126,{ }^{498}$ ISO 10075-2, ${ }^{499}$ ISO 9241-210,500; and ISO/IEC $14598 . .^{501}$

\subsubsection{Instructions, Assistance, and Help}

Examiners work across disparate application interfaces, which can be confusing. Managers should place instructions of all types and formats in locations accessible and visible to employees. Posted instructions should include icons and font sizes that are large enough to see. Forensic service providers should also consider the best ways to present error feedback, assistance, and helpful information to examiners. Although the NIST team did not document instructional training process or online help functions, any instructions or help should follow human factors guidelines and standards.

Online documentation, training, and help can be beneficial. Unfortunately, most of the current online documentation is little more than paper documentation displayed on a computer screen. Designers of online instruction and documentation should consider the delivery technique and should utilize indexes, searches, and other navigational tools, including text, graphics, and icons, to help users find relevant content. ${ }^{502}$ Research on both hard-copy and online documentation suggests that users seldom read the entire document. ${ }^{503}$ Learning styles also influence the user's preference in accessing information. ${ }^{504}$

Many icons used in instructions for examiners do not easily describe the tasks they represent. For some especially obscure icons, even pop-up label descriptions do not assist in comprehension. ${ }^{505}$ Designers should use standard icons whenever possible and should conduct usability testing before launching the prodcut. Applicable standards are ISO/IEC/IEEE $26512^{506}$ and ISO/IEC 11581.507

\footnotetext{
${ }^{498}$ ISO/IEC 9126:2001, op cit.

${ }^{499}$ International Organization for Standardization. Ergonomic Principles Related to Mental Workload-Part 2: Design Principles, ISO 10075-2:1996. International Organization for Standardization, 1996.

${ }^{500}$ ISO 9241-210:2010, op cit.

${ }^{501}$ International Organization for Standardization. Information Technology-Software Product Evaluation, ISO/IEC 14598:1999. International Organization for Standardization, 1999.

502 Moallem, A. "Usability of Software Online Documentation: A User Study." HCI International, 1 (2003): 549.

${ }^{503}$ Rettig, M. "Nobody Reads Documentation." Communications of the ACM, 34 (1991): 19.

${ }^{504}$ Kolb, D. Experiential Learning: Experience as the Source of Learning and Development. Prentice Hall, 1984 ; Felder, R. "Reaching the Second Tier: Learning and Teaching Styles in College Science Education." Journal of College Science Teaching, 23 (1993): 286.

${ }^{505}$ Zammit, K. “Computer Icons: A Picture Says a Thousand Words. Or Does It?” Journal of Educational Computing Research, 23 (2000): 217.

${ }^{506}$ International Organization for Standardization. Systems and Software Engineering-Requirements for Acquirers and Suppliers of User Documentation, ISO/IEC/IEEE 26512:2011. International Organization for Standardization, 2001. Replaces the withdrawn standard I International Organization for Standardization. Information TechnologySoftware User Documentation Process, ISO/IEC 15910:1999 (withdrawn). International Organization for Standardization, 1999.
}

151 Latent Print Examination and Human Factors: Improving the Practice through a Systems Approach The Report of the Expert Working Group on Human Factors in Latent Print Analysis 


\subsubsection{Environmental Factors}

\section{Air Quality}

Air quality refers to the average or extreme temperature and humidity of the location along with any contaminants. It is often difficult to control air temperature and airflow, especially in open office spaces. Examiners' movements between offices and the additional heat resulting from that movement complicate control over air quality. Fortunately, temperature can be controlled with some degree of accuracy depending on the building's heating, ventilating, and air conditioning (HVAC) system. Indoor temperature affects comfort, perceived air quality, sick building syndrome symptoms, and performance. ${ }^{508}$

Suboptimal ventilation rates and higher occupancy density are associated with lower work performance. ${ }^{509}$ Improved perceived air quality reduces the intensity of sick building syndrome symptoms and improves performance. ${ }^{510}$ Latent print unit managers should monitor the airflow and temperature in all examiner workspaces and, if needed, should install desktop task or ambient conditioning systems or air purifying systems. The standards applicable to air quality are OSHA Section III Chapter 2 (general recommendations for indoor air quality problems including ventilation, air treatment, and source controls) ${ }^{511}$ and ISO 16814 (specifying methods to express the quality of indoor air suitable for human occupancy allowing several acceptable target levels of indoor air quality depending on local requirements, constraints, and expectations). ${ }^{512}$

\section{Lighting}

Examiners may experience vision problems, eyestrain, and injury with insufficient or inappropriate lighting. Placing monitors in front of windows increases glare. Glare from nonadjustable and non-directional lighting fixtures and lamps results in squinting. Inadequate levels of light for the task, inconsistent background luminosity, a lack of natural lighting, a lack of window fixtures to control natural light, as well as nonadjustable lamps for use during magnification all can cause glare. However, studies have found that a view out a window is associated with better worker performance, less fatigue, and better self-reported health

\footnotetext{
${ }^{507}$ International Organization for Standardization. Information Technology-User System Interfaces and SymbolsIcon Symbols and Functions, ISO/IEC 11581:2000. International Organization for Standardization, 2000.

${ }^{508}$ Seppänen, O., W. Fisk, and Q. Lei. Effect of Temperatures on Task Performance in Office Environment. Helsinki Univ. of Technology, Laboratory of Heating, Ventilating, and Air-Conditioning, July 2006.

${ }^{509}$ Federspeil, C., G. Lui, M. Lahiff, et al. "Worker Performance and Ventilation: Analyses of Individual Data for Call-Center Workers." Indoor Air, 14 (2002): 41.

${ }^{510}$ Wargocki, P., D. Wyon, J. Sundell, et al. "The Effects of Outdoor Air Supply Rate in an Office on Perceived Air Quality, Sick Building Syndrome (SBS) Symptoms and Productivity.” Indoor Air, 10, no. 4 (2000): 222-36; Fang, L., D. Wyon, G. Clausen, et al. "Impact of Indoor Air Temperature and Humidity in an Office on Perceived Air Quality, SBS Symptoms and Performance.” Indoor Air, 14, supp. 7 (2004): 74-81.

${ }^{511}$ U.S. Department of Labor, Occupational Safety \& Health Administration. OSHA Technical Manual, Section III, Chapter 2, Indoor Air Quality Investigation. Occupational Safety \& Health Administration, 1999.

${ }^{512}$ International Organization for Standardization. Building Environment Design-Indoor Air Quality-Methods of Expressing the Quality of Indoor Air for Human Occupancy, ISO 16814:2008. International Organization for Standardization, 2008.
} 
conditions. ${ }^{513}$ Higher levels of daylight illumination have a positive impact on attention span and short-term memory tests. ${ }^{514}$

Lighting conditions affect individual performance via the visual, circadian, and perceptual systems. ${ }^{515}$ The lighting conditions determine the capabilities of the visual system. The state of the circadian system (sleep-wake cycle) is influenced by the light-dark cycle. And the "message" delivered by the perceptual system is influenced by many factors, including light. ${ }^{516}$ Because the need for varying levels of light greatly affects examiners' ability to complete their tasks quickly, efficiently, and correctly, managers and environment designers should take the following actions:

- Place monitors away from windows to reduce glare and reflections;

- Provide monitor anti-glare screens;

- Tilt the monitor screen to avoid reflections;

- Adjust screen or character background colors (e.g., black characters on a white screen

- will produce less glare than white characters on a blue background);

- Use ceiling fixtures that provide controlled, indirect lighting;

- Install adjustable light level switches;

- Install and use window blinds to control outside light;

- Use task lights positioned to avoid shadows;

- Provide optimal lighting for examiners using loupes; and

- Reduce other brightness sources, such as glossy, reflective paints on walls or highly reflective work surfaces.

Applicable standards include ISO 8995 (interior lighting, including workplace, emergency, and safety lighting). ${ }^{517}$

Noise

Noise interferes with worker performance. ${ }^{518}$ Noise can prevent workers from hearing signals and can interfere with a worker's mental and physical condition. Indeed, noise can be a health hazard. It can produce serious physical and mental performance problems and psychological stress. Workers in departments with high noise levels have more disciplinary actions and absenteeism and less productivity. Noise also appears to affect the quality of work and accident

${ }^{513}$ Collins, B. Windows and People: A Literature Survey, Psychological Reaction to Environments With and Without Windows. U.S. Dept. of Commerce, National Bureau of Standards, 1975; Kaplan, R. and S. Kaplan. The Experience of Nature: A Psychological Perspective. Cambridge University Press, 1989.

${ }^{514}$ Heschong Mahone Group. Windows and Offices: A Study of Office Worker Performance and the Indoor Environment - CEC PIER 2003. 2003.

${ }^{515}$ Leslie, R. “Capturing the Daylight Dividend in Buildings: Why and How?” Building and Environment, 38, no. 2 (February 2003): 381-85.

${ }^{516}$ Boyce, P., C. Hunter, and O. Howlette. The Benefits of Daylight Through Windows. Lighting Research Center, Rensselaer Polytechnic Institute, 2003; Juslen, H. and A. Tenner. "Mechanisms Involved in Enhancing Human Performance by Changing the Lighting in The Industrial Workplace.” International Journal of Industrial Ergonomics, 35 (2005): 843.

${ }^{517}$ International Organization for Standardization, Lighting of Work Places, ISO 8995:2002. International Organization for Standardization, 2002.

${ }^{518}$ Morrison, W., E. Haas, D. Shaffner, et al. "Noise, Stress, and Annoyance in a Pediatric Intensive Care Unit." Critical Care Medicine, 31, no. 1 (2003): 113; Topf, M. "Hospital Noise Pollution: An Environmental Stress Model to Guide Research and Clinical Interventions.” Journal of Advanced Nursing, 31 (2000): 520. 
rates. ${ }^{519}$ Although humans often adjust by ignoring noise, the ear does not stop processing sound. ${ }^{520}$ Distracting noise sources can range from overhead paging systems and equipment alarms to HVAC systems, plumbing, and other employees.

In a majority of the organizations studied, the NIST team observed staff members playing music and having conversations that could be distracting. Materials that absorb sound, such as ceiling and wall materials and carpeting, can mitigate the impact of noise at a modest cost. ${ }^{521}$ Applicable standards are ISO $11201^{522}$ and OSHA $1910 .^{523}$

\subsubsection{Distractions}

\section{Interruptions}

Interruptions of the examination process can introduce error. Interruptions can come from any number or sources, but the design of the workspace can mitigate some of them. ${ }^{524} \mathrm{Such}$ interruptions can halt the examination process or can result in the examiner attempting to manage the interruption while continuing with the examination. After an interruption, an examination might not continue at the same point as it ended.

Conversations are a common form of interruption. Research reveals that in only limited circumstances are people able to perform two tasks concurrently. ${ }^{525}$ Conversations actually demand a high level of cognitive processing to be attentive and to formulate a response. An examiner whose attention is diverted from the primary task becomes vulnerable to making a mistake. ${ }^{526}$

Latent print comparison workstations can be designed as an open space, a semi-private office, or a private office. In the open space or semi-private office design, the examiner is likely to be subject to unsolicited conversation either directed to him or overheard from others in the same area. In a majority of incidents involving cockpit crew errors studied by the National Aeronautics and Space Administration (NASA), the crews reported having been distracted by conversation at

\footnotetext{
${ }^{519}$ Noweir, M. "Noise Exposure as Related to Productivity, Disciplinary Actions, Absenteeism, and Accidents Among Textile Workers." Journal of Safety Research, 15 (1984): 163; Muzammil, M., A. Khan, F. Hasan, et al. "Effect of Noise on Human Performance Under Variable Load in a Die Casting Industry - A Case Study." Journal of Environmental Science Engineering, 46 (2004): 49.

${ }^{520}$ Olaosum, A., O. Ogundiran, and J. Tobih. "Health Hazards of Noise: A Review Article." Research Journal of Medical Sciences, 3 (2009): 115.

${ }^{521}$ National Academy of Sciences, Institute of Medicine, Board on Health Care Services. Keeping Patients Safe: Transforming the Work Environment for Nurses. National Academies Press, 2004.

522 International Organization for Standardization. Acoustics-Noise Emitted by Machinery and EquipmentDetermination of Emission Sound Pressure Levels at a Work Station and at Other Specified Positions in an Essentially Free Field over a Reflecting Plane with Negligible Environmental Corrections, ISO 11201:2010. International Organization for Standardization, 2010.

${ }^{523}$ U.S. Department of Labor, Occupational Safety \& Health Administration. Regulations (Standards - 29 CFR), Part 1910.95: Occupational Noise Exposure. Occupational Safety \& Health Administration, 1991.

${ }^{524}$ Carayon, P., C. Alvarado, and A. Hundt. Reducing Workload and Increasing Patient Safety through Work and Workspace Design. Center for Quality and Productivity Improvement, University of Wisconsin-Madison, 2003.

${ }^{525}$ Rosekind, M., P. Gander, L. Cornell, et al. Crew Factors in Flight Operations X: Alertness Management in Flight Operations Education Module. National Aeronautics and Space Administration, 2001.

${ }^{526}$ Ibid.
} 
the time of the incidents. ${ }^{527}$ The use of private office space significantly reduces the occurrences of interruption due to conversation. While not the preferred arrangement, semi-private designs can offer some protection when arranged so that examiners do not directly face each other.

Examples of other forms of communication that can interrupt the cognitive process include phones, paging systems, and email. It is possible to control each of these in the office design. In this case, operational policies should address when it is permissible to mute such communications systems. The NIST team observed that although examiners were more likely to comment on the distracting effect of telephone and paging systems, greater attention was given to the Internet when it was available.

The fact that an examiner has many responsibilities complicates the issue of interruptions. Serving in a training, supervisory, or management role, being called as a witness for the court, and being on-call to respond to crime scenes may sometimes affect an ongoing examination. Careful management can mitigate the effects through appropriate scheduling. In addition, examiners who also serve as crime scene technicians should be neither required nor permitted to monitor a dispatch radio for purposes of taking calls for service. Many individuals who become accustomed to being on call fail to recognize the cognitive effort required to be attentive to what is being broadcast. Managers should rely on alternative forms of notification, including a screening process by staff members outside the examination work area.

\section{Location}

Location refers to the environmental and physical characteristics of the workspace. Of concern is how the physical environment influences system design and usage. The NIST team observed the impact of location on various aspects, including:

- Too many disparate systems located on multiple workstations and platforms make it difficult to design the system to meet the user's anthropometric and ergonomic requirements;

- Not all examiners work in a laboratory environment - some work in cubicles, others share office space, and some have private offices — which complicates a consistent ergonomic design; and

- Laboratories have increased levels of noise and movement due to multiple processes occurring over multiple workstations.

Since latent print examination is not always limited to a side-by-side comparison capable of being performed in a single confined space, such as a desktop, examiners must interface with multiple systems during the workday. Examiners often use AFIS and digital imaging systems that are not well integrated and that reside on disparate workstations. As a result, routine task completion requires a great deal of movement between workstations, across multiple interfaces, and sometimes between rooms. This activity causes the examiner to move from one space to another carrying case files and evidence, which reduces efficiency and increases the potential for error.

${ }^{527}$ Dismukes, K., G. Young, and R. Sumwalt. Cockpit Interruptions and Distractions: Effective Management Requires A Careful Balancing Act. National Aeronautics and Space Administration, 1998. 
Office designs that situate all necessary equipment and workstations within a single area will mitigate these concerns.

Managers, in consultation with human factors experts, should consider gathering information on the frequency and pattern of examiner movement and behaviors while completing tasks. This could assist in integrating systems through middleware to allow operation via a single interface or station wherever possible. If this is not possible, managers should consider the use of keyboard and monitor switches to allow access to multiple terminals from one workstation to reduce movement across offices.

Stress

When individuals assess their circumstances as causing stress, their bodies automatically begin a series of stress-response mechanisms comprised of physiological, behavioral, and psychological efforts to adapt to the environmental demands. ${ }^{528}$

Depending on the resources available within a particular agency, it is common for examiners to spend significant time in ancillary activities, such as maintaining equipment, managing office supplies, performing data entry tasks, preparing case file folders, inventorying and preparing case files for archived storage, and serving as phone operators. Such responsibilities can lead to inefficiency, decreased worker satisfaction, and frustration.

During the NIST team observations, examiners frequently mentioned that they were responsible for managing information technology (IT) file backups, system or component selection and installation, integration, and troubleshooting. They also noted that IT support staff member scheduled routine systems maintenance at peak work periods. This activity led to reduced productivity, frustration, and potential errors due to the unexpected interruptions while casework was being performed. The impact of these interruptions led to one examiner's decision to move to another laboratory. Managers should interview examiners to determine what workplace characteristics are considered distracting and stressful and should eliminate any unnecessary stressors from the work environment.

\subsection{Summary}

Workspace design has a substantial impact on employees' productivity, errors, and injuries. A usable latent print examination environment takes into consideration the needs of each examiner to develop an environment that improves productivity, reduces the complexity of the work, increases satisfaction, and reduces the risks of errors and injury. ${ }^{529}$ Examiners' office spaces must reflect good ergonomic design. The workspace and equipment must meet the individual examiner's physical and cognitive needs. Systems should be integrated and standardized to reduce the amount of learning required to complete routine tasks over systems interfaces and office locations. The work environment cannot be designed with a one-size-fits-all approach. However, implementing the recommendations in this chapter, employing good ergonomic design

\footnotetext{
${ }^{528}$ Cohen, S. and M. Rodriguez. "Pathways Linking Affective Disturbances and Physical Disorders." Health Psychology, 14 (1995): 374.

${ }^{529}$ Hendrick, H. "Determining the Cost-Benefits of Ergonomics Projects and Factors That Lead to Their Success." Applied Ergonomics, 34 (2003): 419.
} 
principles, and adhering to usability guidelines and standards will improve the environment for the examiners. 


\section{Appendix 7-A: Design Processes}

\section{A.1 User-Centered Design Process}

At its core, user-centered design is based upon the concept of developing usable, useful products. Each user and his context of use must define the user needs. Optimizing safety, comfort, efficiency, effectiveness, and user satisfaction requires an understanding and application of usability and the user-centered design process.

Table 7.2 lists usability goals and provides a short description of each, as adapted from Nielsen's definition of usability quality metrics. It also provides some questions for latent print examiner environment designers to consider. When designing any usable system, it is important to consider these aspects of the user experience.

\begin{tabular}{|c|c|c|}
\hline Usability Goals & Definition & $\begin{array}{c}\text { Latent Print Examiner-Targeted } \\
\text { Questions }\end{array}$ \\
\hline Effectiveness & $\begin{array}{l}\text { Measure of how well a user can } \\
\text { perform a task }\end{array}$ & $\begin{array}{l}\text { Can examiners successfully encode a print for } \\
\text { submission to AFIS? } \\
\text { Can examiners accurately determine the } \\
\text { orientation of the print? }\end{array}$ \\
\hline Efficiency & $\begin{array}{l}\text { Measure of how quickly a } \\
\text { user can perform work and the } \\
\text { accompanying error rate }\end{array}$ & $\begin{array}{l}\text { Are examiners able to quickly accomplish } \\
\text { goals? } \\
\text { Can examiners perform tasks with few errors? }\end{array}$ \\
\hline Satisfaction & $\begin{array}{l}\text { Measure of user attitudes, } \\
\text { perceptions, feelings, and opinions } \\
\text { regarding the environment }\end{array}$ & $\begin{array}{l}\text { How well does the environment avoid inducing } \\
\text { examiner discomfort and frustration? } \\
\text { Are examiners intimidated by the } \\
\text { environment? }\end{array}$ \\
\hline Learnability & $\begin{array}{l}\text { Measure of how quickly a user can } \\
\text { become productive }\end{array}$ & $\begin{array}{l}\text { Can examiners learn how to use the tools and } \\
\text { systems? } \\
\text { How long should it take an examiner to learn } \\
\text { the tools and system? } \\
\text { Are examiners able to use the tools and } \\
\text { systems (to some defined level of competence) } \\
\text { after instructions or training? }\end{array}$ \\
\hline Memorability & $\begin{array}{l}\text { Measure of how well a returning } \\
\text { user forms a mental model of the } \\
\text { environment and remembers how } \\
\text { to use it }\end{array}$ & $\begin{array}{l}\text { If an examiner has used the tool or process } \\
\text { before, can he remember enough to use it } \\
\text { effectively the next time, or does he have to } \\
\text { re-learn it? } \\
\text { How do experienced examiners differ from } \\
\text { infrequent/novice examiners? } \\
\text { After not using the tool or process for a } \\
\text { period of time, how long should it take for the } \\
\text { examiner to become familiar with it again? }\end{array}$ \\
\hline
\end{tabular}

Table 7.2: Definitions of usability goals and questions that apply specifically to latent print examiners $^{530}$

\footnotetext{
${ }^{530}$ Nielsen, op. cit.
} 


\section{A.2 Iterative Process}

Users who are included in the early stages of the design phase can provide feedback and recommendations that designers can incorporate into the initial prototypes. Designers of the most successful environments continually refine their prototypes through an iterative, evolutionary process with users. This iterative process must be user-centered, research-based, and performance-driven.

User-Centered:

- Identifies the types of users who will be using the workspaces, including latent print examiners, forensic specialists, and laboratory managers

- Ensures that the needs of the users are considered in the design and development of the environment

Research-Based:

- Employs research to learn about users and their needs, tasks, environments, level of expertise, etc.

- Conducts ongoing research with examiners by observing them interacting with the workspaces, systems, and hardware

Performance-Driven:

- Utilizes information gathered from users and measures user performance to ensure that design improvements have a measurable impact on users' effectiveness, efficiency, and satisfaction

\section{A.3 Context of Use}

During the first stage of the user-centered design process, the environmental designer must consider the "context of use." The actual conditions under which latent print examination environments are used must be considered to ensure that the design of the environment will meet the needs of users and the objectives of the organization.

Throughout the development process (and especially during the early stages of the design process), designers must be aware of contextual factors that include the business, operational, and social environments in which the system will be used.

\section{A.4 Defining the Users}

The central objective of designing a usable environment is to meet the needs of users within their operational context. The design of the environment should focus on users' needs and expectations, involve users throughout, and integrate feedback from users into the design. Recognizing the full range of users, designers should define the role each user will have within the examiner environment. Designers should try to understand the users' needs, interests, and goals. Table 7.3 lists information about users that can be relevant to the design of the latent print examiner's workspace. 


\begin{tabular}{|c|c|c|}
\hline \multirow[b]{2}{*}{ Characteristics } & \multicolumn{2}{|c|}{ Questions to Consider when Defining Users' Requirements } \\
\hline & Characteristic Specifics & User Requirements \\
\hline Age & What is the age range of this user group? & $\begin{array}{l}\text { Does age impact the use of } \\
\text { technology (e.g., marking digital } \\
\text { images versus photographs)? }\end{array}$ \\
\hline Anthropometrics & $\begin{array}{l}\text { What are the heights of the users? } \\
\text { What are the heights of the workstations? }\end{array}$ & $\begin{array}{l}\text { Are the workstations' heights } \\
\text { adjustable? } \\
\text { Are the chairs' heights adjustable? } \\
\text { Can the users reach the tools required } \\
\text { for the tasks? }\end{array}$ \\
\hline Education & $\begin{array}{l}\text { What is the typical education level of the } \\
\text { users? }\end{array}$ & $\begin{array}{l}\text { How does education affect system } \\
\text { usage? }\end{array}$ \\
\hline $\begin{array}{l}\text { Experience and } \\
\text { Knowledge Level }\end{array}$ & $\begin{array}{l}\text { How much computer experience do the } \\
\text { users have? } \\
\text { How many of the users have experience } \\
\text { with AFIS databases? } \\
\text { How many of the users are new to the } \\
\text { system? } \\
\text { How have users been trained on the } \\
\text { system? }\end{array}$ & $\begin{array}{l}\text { Do the needs of first-time users differ } \\
\text { from those of more frequent users? } \\
\text { Does the system need to } \\
\text { accommodate users with training } \\
\text { needs? }\end{array}$ \\
\hline Visual Attributes & $\begin{array}{l}\text { Do any of the users have color blindness? } \\
\text { Do any of the users have contrast } \\
\text { sensitivity? } \\
\text { Do any of the users have dyslexia? } \\
\text { Do users have other characteristics with } \\
\text { respect to visual acuity or other factors? }\end{array}$ & $\begin{array}{l}\text { How do these attributes affect the } \\
\text { environmental design? }\end{array}$ \\
\hline
\end{tabular}

Table 7.3: Characteristics to consider when designing latent print examiners'work environments

\section{A.5 Designing the User Environment}

Once the characteristics have been defined, designers can begin to consider other environmental factors. For example:

- When and where will users analyze prints, compare prints, evaluate prints, and verify prints?

- When and where will they interact with AFIS databases or other software systems?

- What are the characteristics of the environment, including physical infrastructure; configuration of workstation; placement of workstation; seating; height, angle, and distance from user; lighting; noise levels; frequency of tours; temperature and humidity; placement, type, and format of training materials, policies, procedures, and safety equipment; and the types of help that are available? 
Designers can use Table 7.4 to help them identify how the environment will be used, to define possible user requirements and to ask design questions relating to each element of the workspace and environment.

\begin{tabular}{|c|c|c|}
\hline \multirow[b]{2}{*}{ Characteristics } & \multicolumn{2}{|c|}{ Questions to Consider } \\
\hline & Characteristic Specifics & Potential Effects \\
\hline Location & $\begin{array}{l}\text { In what environment will the } \\
\text { workstation be located? } \\
\text { What are the physical characteristics } \\
\text { of this location? } \\
\text { Within the location, how will the } \\
\text { workstation be situated? }\end{array}$ & $\begin{array}{l}\text { How does the physical } \\
\text { environment of this location } \\
\text { affect or influence the design and } \\
\text { usage of the system? } \\
\text { How does the placement of the } \\
\text { workstation affect use of and } \\
\text { access to the system? } \\
\text { What spatial requirements are } \\
\text { necessary to facilitate frequent } \\
\text { use? }\end{array}$ \\
\hline Temperature and Humidity & $\begin{array}{l}\text { What is the average temperature for } \\
\text { the location? } \\
\text { What are the extreme temperatures? Is } \\
\text { the area humid? }\end{array}$ & $\begin{array}{l}\text { How do temperature and humidity } \\
\text { affect the users? } \\
\text { How does the outside } \\
\text { environment affect performance? }\end{array}$ \\
\hline Lighting & $\begin{array}{l}\text { What types of lighting are utilized? } \\
\text { Will the system be utilized at night as } \\
\text { well as during the day? }\end{array}$ & $\begin{array}{l}\text { How does the level of lighting } \\
\text { affect readability and visibility? }\end{array}$ \\
\hline Noise & What is the average noise level? & $\begin{array}{l}\text { How does the noise level affect an } \\
\text { individual's ability to perform? }\end{array}$ \\
\hline Instructions/Warnings & $\begin{array}{l}\text { Where are instructions placed? } \\
\text { What is the format of instructions } \\
\text { (e.g., signs, labels, icons)? }\end{array}$ & $\begin{array}{l}\text { Are the instructions large enough? } \\
\text { Are the instructions obstructed? } \\
\text { What is the appropriate height to } \\
\text { place instructional guides? }\end{array}$ \\
\hline Assistance & $\begin{array}{l}\text { What types of help and assistance are } \\
\text { provided? }\end{array}$ & $\begin{array}{l}\text { Given the environment, what } \\
\text { is the best way to present error } \\
\text { feedback and helpful information? }\end{array}$ \\
\hline
\end{tabular}

Table 7.4: Characteristics regarding each element of an environment 


\section{A.6 Defining User Goals and Tasks}

After identifying user characteristics and task requirements, designers should conduct a user and task analysis by answering questions such as:

- What is the user's purpose?

- What are the user's needs, interests, and goals?

- How will the user interact with the environment?

- What are the key tasks the user must perform?

- Which tasks will the user perform frequently?

- Which tasks are critical to the user's success in the environment?

- Which tasks are critical to the success of the organization?

Designers should consider how human factors issues affect each task. Important tasks that could be affected by human factors issues should be evaluated first. Designers should consider these questions for each group of users identified in the previous section. 


\section{Chapter 8: Training and Education}

\section{Introduction and Scope}

This chapter reviews the history and current state of latent print examiner training programs, standards, and requirements. It identifies shortcomings in the current system and offers recommendations to improve training through research into and development of effective mentoring programs and comprehensive training materials. The chapter also discusses existing standards of professional organizations, requirements for accreditation, and benefits of certification. It recommends mandatory certification and accreditation to improve the latent print field as a whole.

\subsection{A History of Training in the United States}

The first formal training for latent print examiners in the United States took place during the 1904 World's Fair in St. Louis when Sergeant John K. Ferrier of Scotland Yard's Fingerprint Bureau provided brief "instructions to hundreds of police officers and prison officials." ${ }^{3} 1 \mathrm{He}$ then conducted a 7-month training session for 9 people on the comparison of unknown fingerprint specimens to known exemplars and the classification of fingerprint records. ${ }^{532}$

By 1915, the International Association for Criminal Identification (later, the International Association for Identification, or IAI) was formed. One objective was "to provide training, education, and the publication of information in all forensic disciplines represented by this Association." 533 The Association also instituted an annual training seminar. ${ }^{534}$

In 1916, T. G. Cooke and W. K. Evans created a school in Chicago to offer a home-study course. The school had several names until, in 1926, it became the Institute of Applied Science (IAS). The IAS course proceeded through various functional areas with reading and competency testing. Graduation usually required a year and a half of "study monitored and directed by personal and highly professional, albeit long distance, tutelage. ... By 1937, and continuing for many years after, nearly one half of the individuals in charge of fingerprint bureaus in North America were IAS graduates." ${ }^{335}$

In 1924, the Federal Bureau of Investigation (FBI) created an Identification Division. It offered a 2-week fingerprint examiner training program for law enforcement agencies throughout the country. ${ }^{536}$ The first week of the program dealt with recognizing fingerprint types, identifying characteristics, and classifying fingerprint records. The second week focused on comparing

\footnotetext{
${ }^{531}$ Grieve, D. “The Identification Process: Traditions in Training.” Journal of Forensic Identification, 40 (1990): 199.

${ }^{532}$ Ibid., p. 209.

${ }^{533}$ International Association for Identification. Constitution, Article 1, Section 1.03f. International Association for Identification, 2011.

${ }_{534}$ Grieve, op. cit., p. 206.

${ }^{535}$ Ibid., p. 207.

${ }^{536}$ Federal Bureau of Investigation. The Science of Fingerprints: Classification and Uses. U.S. Department of Justice, 1979.
} 
unknown fingerprint specimens to known fingerprint records. The program concentrated on the development of the skills needed for comparing fingerprints and for developing latent prints. It did not concern itself with the underlying science that made the work plausible or with instruction in scientific methodology.

"Ridgeology," ${ }^{37}$ a holistic approach to the latent print comparison process that goes beyond the counting and arrangement of friction ridge minutiae, required latent print examiners to learn more about friction ridge skin, its discriminating features, and the comparison process. Some larger agencies created their own training programs, but most small providers relied on existing examiners to train new ones informally. Without the leadership of a central authority, no formal training program emerged.

The IAI and the Scientific Working Group for Friction Ridge Analysis, Study and Technology (SWGFAST) have published suggestions concerning training programs for latent print examiners. ${ }^{538}$ Although these documents outline the training needs for examiners, neither organization provides training materials (see section 8.4).

Today, forensic examiners receive training through a wide variety of programs and approaches, and they follow a range of educational pathways that lead to careers in latent print examination. ${ }^{539}$ Latent print examiners may receive some training as part of a formal undergraduate or graduate program in forensic science or criminal justice, or they may have acquired their professional skills through on-the-job training. ${ }^{540}$ Latent print examiners usually work at law enforcement agencies where training comes from internal mentorships, sometimes supplemented by external programs and formal training. In this traditional approach, one generation of trainers transmits its knowledge and skill to the next. ${ }^{541}$

\subsection{Concerns about the Current Training System}

Today's variegated system of training has some benefits, but it lacks uniformity and raises concerns about quality. For example, the 2009 National Research Council report on forensic science in the United States observes that latent print training varies from agency to agency and may include "a formalized training program, may use an informal mentoring process, or may

\footnotetext{
${ }^{537}$ Ashbaugh, D. Ridgeology: Modern Evaluative Friction Ridge Identification. Royal Canadian Mounted Police, 1999.

${ }^{538}$ International Association for Identification. Friction Ridge Skin Identification Training Manual. International Association for Identification, 2006; Scientific Working Group on Friction Ridge Analysis, Study and Technology. Standards for the Minimum Qualifications and Training to Competency for Friction Ridge Examiner Trainees (Latent/Tenprint). Version 1.0. Scientific Working Group on Friction Ridge Analysis, Study and Technology, February 2010.

${ }^{539}$ Gaensslen, R. "How Do I Become a Forensic Scientist?, Educational Pathways to Forensic Science Careers." Analytical and Bioanalytical Chemistry, 376 (2003): 1151-55.

${ }^{540}$ Scientific Working Group on Friction Ridge Analysis, Study and Technology. Strengthening Forensic Science in the United States: A Path Forward, Position Statement. Scientific Working Group on Friction Ridge Analysis, Study and Technology, 2009.

${ }^{541}$ In 1990, David Grieve wrote that "fingerprint training is centered around some type of apprenticeship that contains varying amounts of tutelage, on-the-job training, or self study. Structured, formal courses that provide complete instruction, either academic or employer designed, are rare.” Grieve, op. cit., p. 195.
} 
send new examiners to a 1- to 2-week course." ${ }^{542}$ This lack of standardized training can lead to a wide range of practices. Informal training programs vary in length from weeks to years, and testing for competency may not exist within each department.

Recent years have brought notable efforts to standardize formal academic programs in forensic science; ${ }^{543}$ however, much of the analysis of education and training has focused on forensic science degree-granting programs, neglecting training specific to latent print examination. The absence of enforceable standards has led to heterogeneity in curricula, instructors, pedagogy, documentation, and mentorships. Some agencies do not have staff members available to mentor trainees adequately, and even when mentors are available, it is not clear how they are selected or trained.

Smaller agencies with only a handful of examiners often find it particularly challenging to provide adequate training. Training might be limited to the most practical aspects of the job, as pressures are in place to apprentice and to manage the caseload. Because of the lack of industrywide standards, training is limited to the level of expertise of the trainer, which can lead to perpetuation of improper or inappropriate methods. Limited career paths in smaller agencies can create high turnover rates that make it constantly necessary to train new examiners.

A few professional and private organizations supply advanced training and education. However, there has been little to no effort to evaluate the content of the training to show its effectiveness. ${ }^{544}$ Furthermore, only a fraction of all examiners participate in these courses, leaving the field without uniform standards and requirements for education and training, consistently qualified instructors, and comprehensive and updated curricula.

\subsection{Recommendations for Training}

To improve the training system, it is necessary first to understand what skills and knowledge are necessary for latent print examination. Examiners must have the proper cognitive abilities to conduct latent print examinations and to communicate their findings. Specific abilities in perception and judgment are required for the analysis, comparison, and evaluation of friction ridge impressions. These go beyond screening for form blindness, color blindness, and visual acuity.

\footnotetext{
${ }^{542}$ National Academy of Sciences, National Research Council, Committee on Identifying the Needs of the Forensic Science Community. Strengthening Forensic Science in the United States: A Path Forward. National Academies Press, 2009, p. 136. For more extensive discussions, see Cowger, J. "Moving Towards Professionalization of Latent Print Examiners." Journal of Forensic Sciences, 24 (1979): 591-95; Cooney, L. "Latent Print Training to Competency: Is it Time for a Universal Training Program?" Journal of Forensic Identification, 60 (2010): $223-58$.

${ }^{543}$ Technical Working Group for Education and Training in Forensic Science. Education and Training in Forensic Science: A Guide for Forensic Science Laboratories, Educational Institutions, and Students. National Institute of Justice, 2004; American Academy of Forensic Sciences, Forensic Science Education Programs Accreditation Commission. Accreditation Standards. American Academy of Forensic Sciences, revised July 22, 2011 , Article I. ${ }^{544}$ For additional information on ways to evaluate, see Kirkpatrick, D. and J. Kirkpatrick. Evaluating Training Programs: The Four Levels. 3rd ed. Berrett-Koehler Publishers, 2006.
} 
Recommendation 8.1: The federal government should consider funding research to determine what educational and cognitive abilities should be prerequisites for training a latent print examiner. Forensic service providers should have systematic training programs that develop these abilities as well as educate examiners in the scientific method, the relevant scientific literature, communication skills, and methods for logically developing conclusions.

Once a systematic training program on all aspects of latent print examination is developed, it must be affordable to forensic service providers. Budgetary constraints limit the number of trainees who can be hired, and training through formal programs outside the agency can be costly. Previous federally funded incentive programs, such as the Law Enforcement Education Program (LEEP) and its successor, the U.S. Department of Justice Police Corps program, were designed to address similar concerns from a broader law enforcement perspective. The Police Corps program provided scholarships or tuition reimbursements in return for a 4-year commitment to work as patrol officers in a state or local law enforcement agency. Federal funds went to states to develop and provide training. In addition, the federal government provided local and state agencies that hired Police Corps officers $\$ 10,000$ a year for 4 years of service. Similar incentives to support training, education, and salaries of latent print examiner trainees would encourage agencies to create positions or fill vacancies.

\section{Recommendation 8.2: The federal government should develop a forensic latent print examiner scholarship program to:}

a. Fund the post-hire training program and, upon successful completion, provide tuition reimbursement, loan forgiveness, or other financial assistance to degreed latent print examiner trainees working in state, local, or private (not-for-profit) forensic laboratories; and

b. Reimburse the employer for a percentage of the salaries during the training period.

By learning what specific skills and knowledge are essential to successful latent print examinations and by implementing programs that allow all examiners to receive proper training, forensic service providers can ensure that their examiners have the tools they need and can pass this information on to newer employees.

On-the-job relationships play a critical role in the professional development of new examiners. Such relationships provide opportunities to receive feedback, counseling, coaching, skill building, advancement, role modeling, and reinforcement. ${ }^{545}$ Informal relationships often occur naturally between co-workers. However, formal mentoring programs allow agencies to foster developmental relationships between junior and senior staff members by taking an active role in their initiation by assigning mentors and monitoring this relationship's effectiveness. Organizational benefits of establishing formal mentoring programs include transferring organizational culture and institutional knowledge, reinforcing and encouraging teamwork, reducing stress, and decreasing turnover. ${ }^{546}$ Research shows that successful mentoring programs

\footnotetext{
${ }^{545}$ Kram, K. and M. Bragar. "Career Development Through Mentoring: A Strategic Approach for the 1990s - Part I.” Mentoring International, 5, no. 3 (Summer 1991): 3-13.

${ }^{546}$ Jorgenson, J. Mentoring Programs: An Overview. National Academy of Public Administration, 1992.
} 
include voluntary participation, matching mentors and protégés who are two to three levels apart, careful selection of mentors, flexible duration, one-on-one mentoring, a no-fault exit provision, a high priority on program evaluation, and the clear communication of mentoring objectives. ${ }^{547}$ Poor coordination of mentorship programs can lead to conflicts between the mentoring and supervisory roles of the mentor, breaches of confidentiality, mentor bias, lack of active listening, and role confusion. ${ }^{548}$ Agencies must take care to minimize these risks.

\section{Recommendation 8.3: Agencies should establish formal mentoring programs that define the roles and responsibilities of the mentor, trainee, and supervisor.}

The Working Group appreciates that agencies with limited staff may find it difficult to provide their own mentoring programs, but these agencies may develop cooperative agreements with neighboring jurisdictions to support the program. As in all educational programs, the mentors should be highly qualified. Mentors and instructors, whether in the workplace or in formal training and educational programs, should possess expert subject-matter knowledge that they can convey clearly and effectively.

\subsection{Recommendations for Curriculum and Information Resources}

Although various training materials ${ }^{549}$ and texts ${ }^{550}$ for latent print examiners are published and available, there is a lack of centralized, easily accessible, and readily understandable sources of valid information. This gap leads to a related recommendation.

\section{Recommendation 8.4: The federal government should establish an expert group to develop a latent print educational textbook, practical exercises, and assessment tests (print and online) in consultation with professional organizations, senior latent print examiners, researchers with content knowledge, experienced trainers, and professionals experienced in curriculum development.}

In developing new curricula, trainers should consider the expertise of the targeted audience and the teaching and testing methods used. ${ }^{551}$ Content should be based upon published standards, peer-reviewed articles, and reference works. Terminology should be consistent throughout the materials. The content should extend beyond the biology and analysis of friction ridge skin. Authors should not be confined to experts in latent print examination, but should include experts

${ }^{547}$ Ibid.; Wilson, J. and N. Elman. "Organizational Benefits of Mentoring." The Executive, 4, no. 4 (1990): 88-94.

${ }^{548}$ Taherian, K. and M. Shekarchian. "Mentoring for Doctors: Do Its Benefits Outweigh Its Disadvantages?" Medical Teacher, 30 (2008): e95-e99.

${ }^{549}$ See, e.g., IAI, op. cit.

${ }^{550}$ See, e.g., Saferstein, R. Criminalistics: An Introduction to Forensic Science. Prentice Hall, 2000; Ashbaugh, D. Quantitative - Qualitative Friction Ridge Analysis: An Introduction to Basic and Advanced Ridgeology. CRC Press, 1999; Vanderkolk, J. Forensic Comparative Science: Qualitative Quantitative Source Determination of Unique Impressions, Images, and Objects. Academic Press, 2009; Cowger, J. Friction Ridge Skin: Comparison and Identification of Fingerprints. CRC Press, 1992; Lee, H. and R. Gaensslen, eds. Advances in Fingerprint Technology. 2nd ed. CRC Press, 2001; National Institute of Justice and Scientific Working Group on Friction Ridge Analysis, Study and Technology. The Fingerprint Sourcebook. National Institute of Justice, August 2011.

${ }^{551}$ See, e.g., Tyler, R. Basic Principles of Curriculum and Instruction. University of Chicago Press, 1949. Tyler recommends defining appropriate learning objectives, introducing useful learning experiences, organizing experiences to maximize their effect, and evaluating the process and revising the areas that were not effective. 
on cognitive issues, statistics, and forensic science. Hands-on exercises relating to bias and cognitive processing should be included. Ideally, the effectiveness of the materials should be evaluated before and after they are deployed.

Recommendation 8.5: Training materials should include topics beyond the technical aspects of friction ridge analysis, such as:

a. Documentation of work and case notes and written and oral communication;

b. Professional ethics;

c. Human factors issues such as fatigue, bias, cognitive influences, perceptual influences, and error;

d. Research methods; and

e. Legal aspects of expert testimony.

Existing clearinghouses are an important resource for those undertaking educational and training activities. For example, the National Clearinghouse for Science, Technology and the Law (NCSTL) assembles into a publicly searchable database relevant scientific, technological, and legal resources and a bibliography specific to forensic science content areas, such as the reliability of automated fingerprint identification systems (AFIS). ${ }^{552}$ Indeed, a resource comparable to the National Library of Medicine's PubMed for forensic science could provide further benefits to researchers, especially given the limited accessibility of some forensic science publications. ${ }^{553}$

Recommendation 8.6: The federal government should support a clearinghouse of materials from existing training programs as well as publications on latent print identification.

\subsection{Existing Standards and Accreditation Requirements}

Whatever training programs and materials are developed to supplement the current system, developing national standards and accreditation requirements are essential to demonstrating the effectiveness of training. Currently, professional organizations offer a mix of standards, recommendations, and requirements, but there is no single, national accreditation that indicates quality.

\subsubsection{Standards}

Mandatory national standards for educational requirements and for a training curriculum for the forensic discipline of latent print examination do not exist, although the IAI and SWGFAST outline the minimum requirements of a training program.

In 1977, the IAI developed its Latent Print Certification Program, which includes education, training, and experience requirements leading to written and practical examinations. The IAI

\footnotetext{
${ }^{552}$ For one example of an NCSTL bibliography, see http://www.ncstl.org/education/AFISReliability (accessed November 29, 2011). Presumably, NCSTL tries to include all significant publications. Abstracts or annotations for these materials would enhance the value of the bibliography.

${ }^{553}$ See Mnookin, J., S. Cole, I. Dror, et al. "The Need for a Research Culture in the Forensic Sciences." UCLA Law Review, 58 (2011): 725.
} 
requires a bachelor's degree or years of experience as a prerequisite to certification in latent print examination. Various training courses are offered to help examiners prepare for the exam, but only one provider is affiliated with the IAI. Each IAI local division sponsors its own conferences and training.

In addition, SWGFAST documents provide guidance for training programs, examiner qualifications, proficiency testing, and standardized practices and terminology. ${ }^{554}$ SWGFAST has established recommendations for training program content and participation competency. SWGFAST has also issued standards for friction ridge automation training that focus on image capturing and the history, theory, and operation of AFIS. ${ }^{555}$

There have been calls for new examiners to have a science background. SWGFAST also recommends that latent print examiners possess a bachelor's degree with some "science-related coursework." ${ }_{556}$ It has been argued that forensic service providers must spend more time and resources to train applicants who lack this background. ${ }^{557}$

\subsubsection{Requirements for Accreditation}

The American Society of Crime Laboratory Directors/Laboratory Accreditation Board (ASCLD/ LAB), Forensic Quality Services, the American Association for Laboratory Accreditation, and other private organizations accredit forensic service providers. In response to a 2004 report from the National Institute of Justice, ${ }^{558}$ the American Academy of Forensic Sciences created a standing committee known as the Forensic Science Education Programs Accreditation Commission (FEPAC). ${ }^{559}$ This Commission "accredits forensic science education programs that lead to a bachelor's or master's degree in forensic science or in natural science with a forensic science concentration." 560 The degree programs must be "located in a regionally accredited institution of higher education." 561

ASCLD/LAB requires agencies to have a documented training program and to follow it; however, there is no guidance as to what the training curriculum should encompass. In a 2009 survey, $75 \%$ of the agencies responding reported a formal written training program in compliance with accreditation requirements. ${ }^{562}$ The survey found no significant differences between accredited and non-accredited agencies with regard to aspects of training, such as the number of comparisons needed, the period of supervised case review, pass-fail policies, and the existence of a dedicated trainer. However, the low response rate for the survey and the possible lack of statistical power make these results difficult to interpret.

\footnotetext{
554 Ibid.

${ }^{555}$ Scientific Working Group on Friction Ridge Analysis, Study and Technology. Standard for Friction Ridge Automation Training (Latent/Tenprint). Version 1.0. Scientific Working Group on Friction Ridge Analysis, Study and Technology, May 2009.

${ }^{556}$ SWGFAST, 2010, op. cit.

557 Stoney, D. “A Medical Model for Criminalistics Education.” Journal of Forensic Sciences, 33 (1988): $1086-94$.

558 Technical Working Group for Education and Training in Forensic Science, op. cit.

${ }^{559}$ American Academy of Forensic Sciences, Forensic Science Education Programs Accreditation Commission, op. cit., section 1.3.

${ }^{560}$ Ibid., section 1.4.

561 Ibid.

${ }^{562}$ Cooney, op cit.
} 


\subsection{Recommendations for Certification and Continuing Education}

Professional certification and accreditation programs typically require some form of continuing education. Continuing education opportunities in the forensic sciences include courses, professional conferences and seminars, distance learning, apprenticeships, residency, internships, teaching, and independent learning. ${ }^{563}$ Latent print examiners should be encouraged to pursue opportunities for such continuing professional education.

Ongoing education that enhances and improves current latent print work activities could include an introduction to cognitive processes, statistical data on fingerprint features, basic probability theory, technological developments, and the ethical and legal responsibilities of latent print examiners. Management should be aware of the skills and expertise of examiners in order to identify gaps that could be eliminated through continuing education and training.

Two major impediments to continuing education programs are cost and access. If programs are not located nearby, or if the costs of enrollment are too high, some examiners will be precluded from continuing education opportunities. To address this issue, the National Institute of Justice provides funding for the development and delivery of online continuing education for forensic scientists and professionals at various levels of knowledge and practice. ${ }^{564}$

\section{Recommendation 8.7: Forensic service providers should require personnel to participate in continuing education and to become certified through an accredited program. Certifying bodies should review current certification tests with the aid of professionals in test design and psychometrics.}

Some jurisdictions have moved toward requiring certification of examiners and accreditation of laboratories. ${ }^{565}$ By earning certification, examiners demonstrate their professional competence and set a knowledge and experience base for themselves, for their employers, and for the public at large. Additionally, certification has benefits beyond those provided to the individual examiner.

- Team performance increases each time a new team member is certified;

- The level of skill that a team has directly affects its performance;

- Certification standardizes practices within the industry and leads to more cooperation among organizations;

- Individuals who earn certification have shown their dedication to personal accomplishment and improvement and may increase their career opportunities; and

\footnotetext{
${ }^{563}$ Technical Working Group for Education and Training in Forensic Science, op. cit.

${ }^{564}$ U.S. Department of Justice, National Institute of Justice. "Training." Accessed November 29, 2011. http://www.nij.gov/training/welcome.htm; RTI International. "Forensic Science Education: Web-Based Professional Development and Continuing Education for Forensic Scientists." Accessed November 29, 2011. http://www.rti.org/page.cfm/Forensic_Science_Education.

${ }^{565}$ New York, Oklahoma, and Texas have state accreditation requirements. National Conference of State Legislatures. Strengthening Forensic Science Oversight. National Conference of State Legislatures, February 2010.
} 
- The public has more confidence in individuals who are certified as meeting some quality standard. ${ }^{566}$

By requiring certification of examiners, forensic service providers can ensure and demonstrate that their employees are competent, knowledgeable, and skilled at all aspects of the latent print examination process.

\subsection{Evaluation of Training Programs}

Although FEPAC evaluates and accredits forensic science curriculum in degree-granting academic institutions, other forensic science training programs do not receive review or evaluation. Laboratory accreditation and examiner certification programs include educational and training requirements. However, these requirements only verify that education and training have occurred; they do not assess the quality of the training programs. ${ }^{567}$

Certifying and accrediting bodies should evaluate training material and training programs. Evaluation methods could include student surveys, measurements against defined learning objectives, or documentation of outcomes. Professional organizations offering training should actively seek a means for external evaluation of their programs.

\section{Recommendation 8.8: Certifying bodies should ensure that examiners have been trained to competency. Laboratory accrediting bodies should evaluate the effectiveness of an agency's training program.}

\subsection{Summary}

Although some training programs and informational resources exist to educate latent print examiners, improvement will require a shift to a single, national standard training system that covers more than the technical tasks involved in latent print examination. A comprehensive training program should include information about report writing, testifying, maintaining professional and personal ethics, understanding the influence of cognitive bias, and calculating statistics. Certification and accreditation organizations should review the effectiveness of new training programs, and forensic science providers should require their examiners to earn certification to demonstrate their commitment to quality.

\footnotetext{
${ }^{566}$ Microsoft Learning Whitepaper. The Value of Certification: Connecting the Dots Between Employers and Employees. Microsoft Corporation, March 2007; SeaCrest Company. "The Value of Certification." Credentialing Talk, August 1, 2007. Accessed October 21, 2011. http://seacrestcompany.blogspot.com/2007/08/value-ofaccreditation.html.

${ }^{567}$ For additional discussion of certification, see Chapter 9.
} 


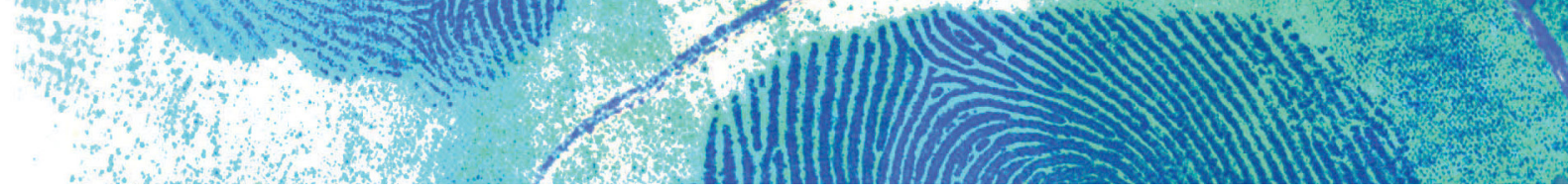

\section{Chapter 9: Human Factors Issues for Management}

\section{Introduction}

The primary responsibility of management is to develop policies and to promote a culture for successful completion of tasks. Other chapters in this report focus on factors that affect the quality of the work in a latent print unit. These include the work environment (Chapter 7), skills and expertise in interpreting prints (Chapter 3), technology (Chapter 4), reporting and testimony (Chapters 5 and 6), and personnel training (Chapter 8). Management plays a central role in all those areas by establishing strategies and a culture that recognizes and responds to human factors in latent print examination.

Managers must be committed to developing, maintaining, and improving quality assurance (QA) and quality control (QC) processes. QA includes procedures to ensure that final results are valid, and QC includes monitoring to verify that procedures are working. ${ }^{568}$ Taken together, QA and QC constitute a quality management system. This chapter focuses on the responsibilities of management in creating an environment that enhances quality through certification, accreditation, and proficiency testing as well as a systems approach to reducing errors.

\subsection{A Management Perspective on Errors}

Chapter 2 describes the types of errors that can occur in the complex endeavor of latent print examination. That chapter used four categories for the possible outcomes of a latent print examination - identification, inconclusive, exclusion, and not suitable for comparison (insufficient data). However, forensic service providers differ in the number and names of possible outcomes of latent print examinations. This chapter uses a more elaborate typology with five categories: identification, inclusion, inconclusive, exclusion, and not suitable.

In casework, examiners do not start with knowledge of the truth. They reach conclusions based upon knowledge, training, and experience. The quality management system should continually evaluate performance. Part of this system is the review of the examiner's results by another qualified examiner. If there is conflict, the agency must employ a pre-determined procedure for resolving the conflict. Having determined the result judged to be "correct," the agency would attribute an "error" to one of the examiners. For example, the two images in Figure 9.1 would produce a very complex comparison that could generate different results from different examiners.

${ }^{568}$ Deming, W. Out of the Crisis. Massachusetts Institute of Technology, Center for Advanced Educational Services, 1986. 


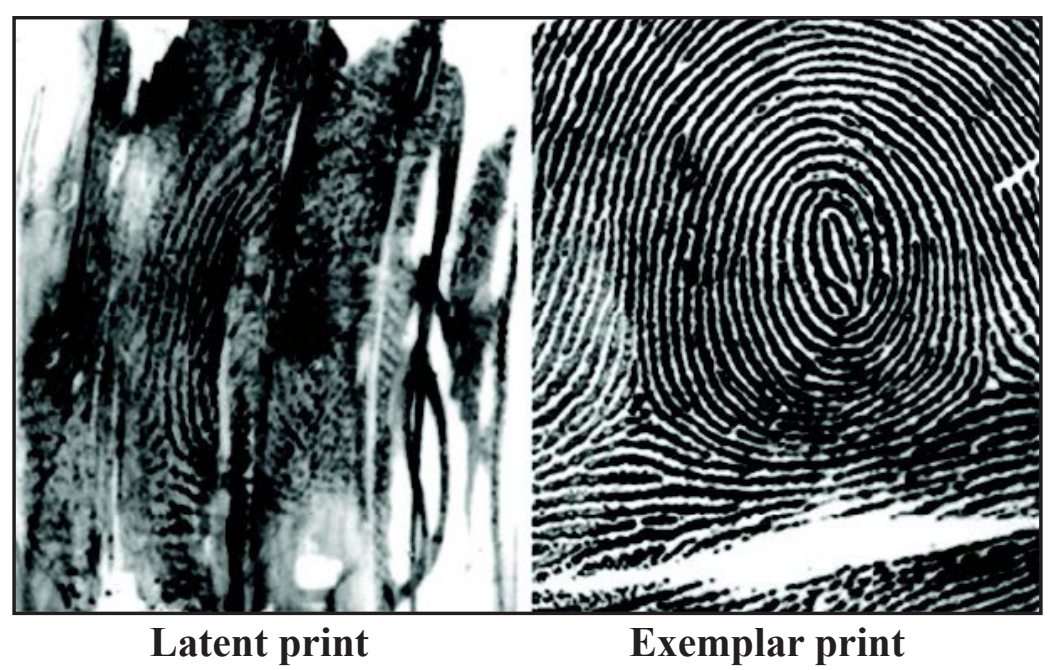

Figure 9.1: Latent print from an ashtray and an exemplar print ${ }^{569}$

Presented with this pair of prints, five different examiners might each report a different conclusion, as shown in Table 9.1.

\begin{tabular}{|l|l|l|}
\hline Examiner & \multicolumn{1}{|c|}{ Result } & \multicolumn{1}{c|}{ Explanation } \\
\hline 1 & Identification & $\begin{array}{l}\text { The examiner determined that there is enough similarity between the } \\
\text { latent and exemplar to make an identification. }\end{array}$ \\
\hline 2 & Inclusion & $\begin{array}{l}\text { The examiner found detail in agreement; however, due to the limited } \\
\text { quality of the latent print, a conclusive identification was not possible. } \\
\text { The examiner also determined that selection of additional print details } \\
\text { would not result in a more definitive conclusion. }\end{array}$ \\
\hline 3 & Inconclusive & $\begin{array}{l}\text { The examiner could not determine if there was sufficient agreement } \\
\text { between the latent print and the exemplar print but believes that } \\
\text { additional exemplars could aid in the assessment. }\end{array}$ \\
\hline 4 & Exclusion & $\begin{array}{l}\text { The examiner found similarities between the latent print and the } \\
\text { exemplar print but interpreted the distortion artifacts in the latent print } \\
\text { as actual friction ridge detail and determined that these two impressions } \\
\text { came from different sources. }\end{array}$ \\
\hline 5 & Not Suitable & $\begin{array}{l}\text { The examiner determined the latent print was not suitable for comparison } \\
\text { and therefore never compared the latent print to any exemplars. }\end{array}$ \\
\hline
\end{tabular}

Table 9.1: Five possible outcomes of the comparison of the latent and exemplar prints in Figure 9.1

If the two prints come from the same source, the conclusions of examiners 3,4 , and 5 may preclude the conviction of a criminal. If the impressions did not originate from the same source, then the conclusions reported by examiners 1 and 2 may contribute to the arrest and prosecution of an innocent person. Procedures that permit an agency to determine which results are most

\footnotetext{
${ }^{569}$ Image adapted courtesy of the Las Vegas Metropolitan Police Department.
} 
likely to be correct, to detect departures from those results, and to investigate the factors that led to the deviations are critical to the quality management process.

Table 9.2 enumerates the various possible erroneous outcomes in the above example. "Error" in the table is defined relative to the outcome judged to be most acceptable rather than relative to the unknown states of nature (see Chapters 2 and 3).

\begin{tabular}{|c|c|c|c|c|c|c|}
\hline & \multicolumn{5}{|c|}{ Result Judged To Be Correct } \\
\hline & & Identification & Inclusion & Inconclusive & Exclusion & Not Suitable \\
\hline \multirow{5}{*}{ 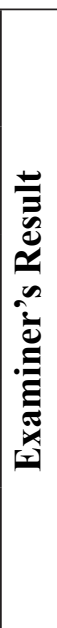 } & Identification & CORRECT & $\begin{array}{l}\text { Erroneous } \\
\text { Identification }\end{array}$ & $\begin{array}{l}\text { Erroneous } \\
\text { Identification }\end{array}$ & $\begin{array}{l}\text { Erroneous } \\
\text { Identification }\end{array}$ & $\begin{array}{l}\text { Erroneous } \\
\text { Identification }\end{array}$ \\
\hline & Inclusion & $\begin{array}{l}\text { Failure to } \\
\text { Identify }\end{array}$ & CORRECT & $\begin{array}{l}\text { Erroneous } \\
\text { Inclusion }\end{array}$ & $\begin{array}{l}\text { Erroneous } \\
\text { Inclusion }\end{array}$ & $\begin{array}{l}\text { Erroneous } \\
\text { Inclusion }\end{array}$ \\
\hline & Inconclusive & $\begin{array}{l}\text { Failure to } \\
\text { Identify }\end{array}$ & $\begin{array}{l}\text { Failure to } \\
\text { Include }\end{array}$ & CORRECT & $\begin{array}{l}\text { Failure to } \\
\text { Exclude }\end{array}$ & $\begin{array}{l}\text { Erroneous } \\
\text { Suitability } \\
\text { Determination }\end{array}$ \\
\hline & Exclusion & $\begin{array}{l}\text { Erroneous } \\
\text { Exclusion }\end{array}$ & $\begin{array}{l}\text { Erroneous } \\
\text { Exclusion }\end{array}$ & $\begin{array}{l}\text { Erroneous } \\
\text { Exclusion }\end{array}$ & CORRECT & $\begin{array}{l}\text { Erroneous } \\
\text { Exclusion }\end{array}$ \\
\hline & Not Suitable & $\begin{array}{l}\text { Failure to } \\
\text { Identify }\end{array}$ & $\begin{array}{l}\text { Failure to } \\
\text { Include }\end{array}$ & $\begin{array}{l}\text { Erroneous } \\
\text { Suitability } \\
\text { Determination }\end{array}$ & $\begin{array}{l}\text { Failure to } \\
\text { Exclude }\end{array}$ & CORRECT \\
\hline
\end{tabular}

Table 9.2: Concordance table listing possible "errors" for conclusions in Table 9.1

The agency's quality management system should track the errors listed above. When errors (either in the sense defined above or relative to ground truth) are detected, it is critical to understand the factors (see appendices) that led to them. By strengthening the quality management system to guard against those factors, the risk of errors can be reduced.

\subsection{A Systems Approach to Improving Accuracy}

From the systems perspective outlined in Chapter 2, human error in the workplace is not an isolated action of a given individual. Rather, it is the result of a chain of events that cumulate in the adverse event. Since Heinrich's 1931 publication, industry has embraced a sequential theory of human error. ${ }^{570}$ James Reason's 1990 "Swiss cheese" model examines error systematically. It assumes that there are fundamental elements of all organizations that must work together harmoniously to achieve efficient and safe operations. ${ }^{571}$ Taken together, these elements comprise a "productive system" as depicted in Figure 9.2, an adaptation of Reason's model.

\footnotetext{
${ }^{570}$ Heinrich, H., D. Peterson, and N. Roos. Industrial Accident Prevention: A Safety Management Approach. 1 st ed. 1931.

${ }^{571}$ Reason, J. Human Error. Cambridge University Press, 1990.
} 


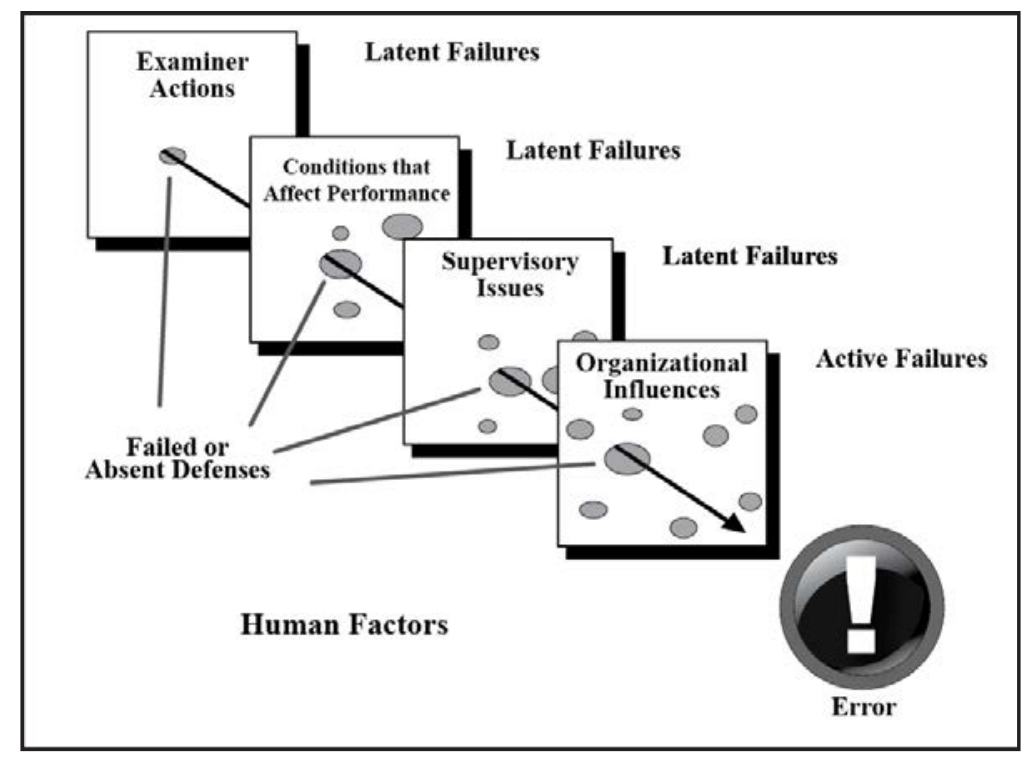

Figure 9.2: Components of a productive system adapted to a latent print unit ${ }^{572}$

For our purposes, the four slices from Reason's original model have been renamed as Examiner Actions, Conditions that Affect Performance, Supervisory Issues, and Organizational Influences. Examiner actions are the mistakes or violations by the examiner. They are what the examiner did. Conditions that affect performance are factors related to the condition of the examiner, communication, or the environment that influenced the examiner. Supervisory issues are any failures in the supervision of the examiner. Lastly, organizational influences are management decisions and the organizational climate that affect the examiner. All require analysis and discussion, and Appendix A lists examples of actions in each category.

Based upon this model, forensic analysis can be viewed as a complex system whose product is the interpretation of forensic evidence. Productive activities within a latent print unit require reliable, well-maintained equipment and a well-trained professional workforce. These are included in the Conditions that Affect Performance slice in Figure 9.2. Examiners also need good management and effective supervision, and managers need appropriate guidance, personnel, and funding to perform their duties, which is represented in the Supervisory Issues slice. The support of management comes from decision-makers who set goals and manage available resources, represented by the Organizational Influences slice. They must balance oft-competing goals of throughput, due diligence, and resources. These executive decisions typically are based upon social, economic, and political input from outside the organization as well as on feedback from managers and workers within it.

Accidents occur when there are breakdowns in the interaction among the components in the production process. ${ }^{573}$ These failures, shown as holes in the different slices, make the system more vulnerable to error, especially when there are multiple failures at each level. Using the

\footnotetext{
${ }^{572}$ Image adapted from Reason, op. cit.

${ }^{573}$ Ibid.
} 
"Swiss cheese" model of error causation, an error occurs when the holes from each slice are aligned.

There are two necessary ingredients to identifying weaknesses in a forensic system: (1) a human error model to capture and organize the information and (2) forensic experts to analyze the examination process and to identify the human and other factors that can affect the outcome. The literature contains many human error models. ${ }^{574}$ If the underlying assumptions regarding the nature and cause of error are considered, there are six different perspectives to error investigation: cognitive, ergonomic, behavioral, medical, psychosocial, and organizational. ${ }^{575}$ Each perspective on human error investigation has its advantages. Many industries therefore have employed a multi-perspective approach, using models such as root cause analysis, failure mode and effects analysis, a management oversight risk tree, a safety management organization review technique, and the human factors and analysis classifications system (HFACS). This chapter uses HFACS as a framework for discussion, ${ }^{576}$ but other error models would elucidate the same factors

\subsubsection{Examiner Actions}

Problematic actions of the examiner can be loosely classified as either mistakes or violations. Mistakes represent normal behavior that fails to produce the desired outcome. Violations refer to the willful disregard of accepted practices. These mistakes and violations should be considered in more detail.

Decision-, Skill-, and Perception-Based Mistakes

Decisions are based primarily on three factors: information, knowledge, and experience (see Chapter 3). In latent print examination, information lies in the latent and exemplar prints, which must be of sufficient quality and quantity to compare and evaluate. In addition, the examiner should be provided with other information, such as the surface from which the latent prints were recovered, the technique used to develop and recover the latent prints, and the method used to capture the exemplar prints. The examiner applies training, background knowledge, and experience comparing a broad range of pairs of latent and exemplar prints to assess the available information. When important information, knowledge, or experience is lacking, mistakes can occur. Often referred to as honest mistakes, these mistakes typically present themselves as poorly executed procedures, improper choices, or the misinterpretation or misuse of relevant information.

Other mistakes occur with little or no conscious thought. For instance, during latent print examination, examiners can orient many latent prints without conscious attention. Tasks such as these highly practiced and automatic behaviors are particularly affected by attention or memory failures. Distractions in the laboratory (see Chapter 7) may lead to a loss of concentration, erroneous documentation, and other mistakes.

\footnotetext{
${ }^{574}$ Senders, J. and N. Moray. Human Error: Cause, Prediction, and Reduction. Psychology Press, 1991.

${ }^{575}$ Wiegmann, D. and S. Shappell. A Human Error Approach to Aviation Accident Analysis: The Human Factors Analysis and Classification System. Ashgate Publishing, 2003.

${ }^{576}$ Ibid.
} 
Additionally, mistakes can occur as a result of the manner in which the examiners store and compare latent print data. For instance, when latent and exemplar prints have only limited corresponding information, the examiner must search different regions of the latent print separately, breaking up the pattern of data and searching each component. Examiners who are inclined to search the latent print as a complete image (searching for the whole pattern) can fail to identify the latent print or can erroneously exclude the exemplar.

Examples of these types of mistakes in latent print examination are failures to find target data, to properly weight the data, to recognize distortion, and to compare enough corresponding features. These types of mistakes can lead to a conclusion exceeding the abilityof the examiner, a failure to search all exemplars, a hurried or insufficiently thorough examination, insufficient data to support the conclusion, a misprioritized level of effort, and improperly deeming a print to be suitable or unsuitable.

\section{Box 9.1: Learning from Others: Benchmarking in Forensic Science}

Forensic laboratories and latent print examiners can learn from each other and from other industries to improve procedures and human factors considerations. This process, called benchmarking, is primarily associated with actions that Xerox took in the early 1980s to remain competitive with companies that were producing less expensive and higher quality copiers. Xerox not only studied its competitors' products and processes, but the company also looked at other industries' processes and translated those best practices into the Xerox standard procedures. Xerox defines benchmarking as "the continuous process of measuring our products, services, and practices against our toughest competitors or those companies known as leaders." ${ }^{577}$ This model is comprised of ten steps in four phases:

Planning phase

Select a subject to benchmark

Identify the best practitioners in the field of study

Determine the data-collection method and collect the data

Analysis phase

Determine the current gap

Project future performance

Integration phase

Communicate the results of analysis

Action phase

Establish functional goals

Develop action plans

Implement plans and monitor results

Recalibrate benchmark ${ }^{578}$

In the field of emergency medicine, efforts are underway to incorporate benchmarking. ${ }^{579}$ The non-profit Emergency Department Benchmarking Alliance spearheads this effort by maintaining a database of performance metrics, by hosting conferences and meetings, and by providing community-building services to their member organizations. ${ }^{580}$ According to the Alliance's data, benchmarking seems to be working. Wait times in emergency departments from 2008 to 2009 fell for the first time in four decades. ${ }^{51}$

\footnotetext{
${ }^{577}$ Camp, R. Business Process Benchmarking: Finding and Implementing Best Practices. ASQC Quality Press, 1995.

${ }^{578}$ American College of Emergency Physicians. Benchmarking in Emergency Medicine, March 1997. Accessed November 29, 2011. http://www.acep.org/content.aspx?id=34362.

${ }^{579}$ Ibid.

${ }^{580}$ Emergency Department Benchmarking Alliance. About Us. Accessed November 29, 2011. http://edbenchmarking.org/index.php.

${ }^{581}$ Augustine, J. "ED Wait Times Improving for the First Time in 40 Years." Improving Health Care, Press Ganey. February 21, 2011.
} 


\section{Violations}

Two types of violations deserve mention: willful noncompliance and exceptional violations. Often referred to as "bending the rules," willful noncompliance tends to be habitual and is enabled by a system of supervision and management that tolerates departures from standard procedures. Just as some drivers may go 5 miles per hour over the speed limit, some examiners may engage in shortcuts to come to a conclusion. Exceptional violations are willful and particularly egregious, and they are neither tolerated by management nor routinely observed in the forensic laboratory. An exceptional violation would be akin to driving 30 miles per hour over the speed limit.

Examples of violations include deeming a latent print not suitable to avoid having to compare it, disregarding aspects of the QA/QC process (for example, skipping the Verification phase), intentionally misidentifying a latent print, making an identification or exclusion of a latent print that the examiner knows is not suitable for comparison, reporting results without conducting a comparison, and coercing a verifier into agreeing with a rendered conclusion.

\subsubsection{Conditions that Affect Performance}

Conditions of the examiner, of communication, and of the environment can affect an examiner's performance.

\section{Physical and Mental State}

An examiner's mental state, physiological state, and physical or mental limitations all can affect performance. Adverse mental states include temporary conditions such as exhaustion and stress. The examiner and management should take appropriate action when the examiner is not fit for duty. In most forensic units, examiners confront large backlogs. Some examiners are held to quotas or unrealistic turn-around times. In this environment, examiners could become more concerned with case output than the quality of the work. As a result, examiners may hurry through cases, taking shortcuts in the analysis and documentation, and failing to reach an appropriate conclusion. Plainly, many factors can affect the mental state of the examiner. Examples are anger, apprehension about reaching conclusions, boredom and complacency, distraction, expectancy, fatigue, overconfidence, peer pressure, and personal problems.

In addition, the examiner's physiological state can affect the examination process. For example, the typical latent print examiner is usually bending over a desk or workbench for long stretches of time, looking through a magnifier, and making numerous comparisons. These working conditions produce strain on the neck, back, and eyes. Glare from computer displays and the sheer number of comparisons can result in headaches or eyestrain (see Chapter 7 for more information about healthy workplace design).

Performance can also suffer due to lack of sleep. In many agencies, an examiner could be called to a crime scene in the middle of the night and then be expected to work a normal latent print caseload the next day without rest. Illness can have detrimental effects, and medications can influence a person's quality of sleep and daytime alertness. Other factors bearing on physiological states include alcohol and drug use, nutrition, and injuries. 
Finally, physical or mental limitations affect the condition of the examiner. Deteriorating eyesight is a physical limitation, while an inability to maintain competency is a mental limitation. If the physical or mental limitation cannot be compensated for, the examiner may no longer be qualified to perform latent print examinations. Other physical or mental limitations include chronic psychological disorders, dyslexia, inadequate training or experience, incompatible aptitude, and visual limitations such as poor acuity, poor contrast sensitivity, and color blindness.

\section{Communication}

An examiner must work with other examiners, management, investigators, attorneys, and administrative personnel. While verbal communication is certainly important, communication via case documentation is also imperative. Only with sufficient documentation and reporting can other examiners and supervisors adequately provide technical and administrative review. For instance, understanding the location, orientation, surface, and development technique of the latent print can be critical for interpreting print distortion. Additionally, understanding how the examiner searched the latent print (distal orientation and anatomical region) can provide critical information if an error has occurred.

Communication can suffer from confusing or conflicting directions or demands, a failure to convey or obtain adequate information, lack of report-writing skills, lack of teamwork, poor case documentation, and departures from standard terminology.

\section{Environment}

Environmental factors can contribute to errors. Both the operational environment (e.g., workplace design or clutter) and the ambient environment (e.g., temperature, noise, and lighting) can degrade performance. For example, the intensity, type, and direction of lighting can influence what an examiner perceives as key data. Chapter 7 discusses such physical environmental conditions as clutter, excessive heat or cold, lighting, ventilation, and noise, as well as technical environmental factors such as equipment and software.

\subsubsection{Supervisory Issues}

If an error has occurred, the investigation of the cause(s) starts with the examiner's actions, proceeds through the conditions that may have contributed to the error, and continues on to supervisory actions and possibly organizational oversights or failures.

\section{Leadership}

Effective management provides examiners with adequate training, professional guidance, oversight, and operational leadership. Without this leadership, the chance of errors is greater. Managers can become so overwhelmed with meetings and paperwork that they fail to provide sufficient oversight. Yet, some supervisors take oversight to extremes, becoming too controlling and more concerned with minute details than the accuracy of the work. Micromanaging examiners can delay decision-making, restrict information flow, and diminish confidence and efficiency.

Effective leadership helps avoid errors by ensuring appropriate training; setting a proper example; tracking and assessing job qualifications or skills; monitoring work; providing appropriate feedback, mentoring, and incentives; and maintaining realistic expectations.

179 Latent Print Examination and Human Factors: Improving the Practice through a Systems Approach The Report of the Expert Working Group on Human Factors in Latent Print Analysis 


\section{Operational Planning}

Management is responsible for planning the operations of the forensic unit. Scheduling should include breaks and should take caseloads and deadlines into account. The supervisor who assigns a large complex case to a less experienced examiner may inadvertently set up the examiner for failure. Conversely, burdening the top performers with excessive work can keep them from performing optimally and can limit the opportunities for less experienced examiners to learn.

Many latent print units are so overwhelmed that supervisors must divide casework into rush cases and routine ones. Examiners with many rush cases can feel overwhelmed, frustrated, and confused. Supervisors and managers need ways to allocate casework to maintain productivity without causing frustration. Allowing examiners to finish one batch of cases before assigning another batch can be helpful. Also, management can sometimes request a certain amount of time from the court for rush cases to ensure that examiners can work the case properly. Trials can even be rescheduled, if necessary. Other examples of operational planning failures are not allowing adequate rest breaks; setting conflicting objectives, goals, or standards; and giving unclear or conflicting assignments.

\section{Problem Correction}

When problems are known, they should be corrected. Consistent failure to correct or discipline inappropriate behavior may foster a dysfunctional work environment. This caution also applies to issues associated with equipment and supplies. When necessary repairs are overlooked or supplies do not meet specifications, errors can result.

\section{Supervisory Violations}

Supervisory violations encompass the disregard of existing rules and regulations. An obvious example of poor supervisory behavior is putting undue influence on an examiner to reach a desired result. A more subtle violation is permitting an unqualified examiner to perform casework. Likewise, pushing an examiner to work unreasonably fast or encouraging "bending the rules" and procedures in the interest of completing a case are poor supervisory actions.

\subsubsection{Organizational Influences}

Organizational influences are the fourth and final layer - the last slice of Swiss cheese - to investigate when evaluating the factors that led to an error. Three areas of organizational failures fall into this category: resource management, organizational climate, and operational processes.

\section{Resource Management}

Resource management refers to the management, allocation, and maintenance of organizational resources, including human resource management (selection, training, staffing), budgets, logistics, and equipment design. Management decisions about such resources should focus on both quality and cost-effectiveness. Unfortunately, quality improvements and training are often the first items to be cut when experiencing financial difficulty. Resource management issues include maintaining hiring, evaluation, and promotion policies; matching qualifications to job assignments; reducing costs and managing unfunded directives; providing logistical support; and making suitable equipment available. 


\section{Organizational Climate}

Organizational climate influences examiner performance. The structure of the organization includes the chain of command, delegation of authority and responsibility, communication channels, and formal accountability for actions. Agency policies that are ill defined, adversarial, conflicting, or supplanted by unofficial rules and values can cause confusion, reduce quality, and lead to a negative organizational climate. Inaccessibility of upper management, inadequate accountability for actions, poorly defined or articulated organizational values, inappropriate allocation of resources, and unclear or conflicting assignments of responsibility can also lead to a negative organizational climate.

Many analysts work in fear of retribution from management, coworkers, and the International Association for Identification (IAI) Certification Board should they misidentify a print. The IAI Certification Program Operations

Manual states that a technical error

may result in suspension or revocation of certification and that the IAI can publish the results of any disciplinary action. ${ }^{588}$ Consequently, some examiners may be unable or unwilling to make difficult identifications. Worse yet, examiners may feel compelled to conceal mistakes.

\footnotetext{
${ }^{582}$ Roberts, K. and R. Bea. "Must Accidents Happen? Lessons from High-Reliability Organizations.” Academy of Management Executive, 15, no. 3 (2001): 70.

${ }^{583}$ Roberts, K. "Some Characteristics of One Type of High Reliability Organization.” Organization Science, 1 , no. 2 (1990): 160.

${ }^{584}$ Baker, D., R. Day, and E. Salas. "Teamwork as an Essential Component of High-Reliability Organizations." Health Services Research, 41 (August 2006): 1576-98.

${ }^{585}$ Dyck, L. "High Reliability Organization (HRO) in Practice.” Technical Support. January 2007.

${ }^{586}$ Rochlin, G. “Defining 'High Reliability’ Organizations in Practice: A Taxonomic Prologue.” In New Challenges to Understanding Organizations. Macmillan, 1993.

${ }^{587}$ Roberts and Bea, op. cit.

${ }^{588}$ International Association for Identification. Certification Program Operations Manual. Version 2010_H_I. International Association for Identification, July 10, 2010.
}

181 Latent Print Examination and Human Factors: Improving the Practice through a Systems Approach The Report of the Expert Working Group on Human Factors in Latent Print Analysis 
Effective management is vital to delivering high-quality forensic services. Because human error in interpreting forensic science evidence can have devastating effects, examiners should be encouraged to come forward to help understand when and why errors occur. Management must foster a culture that promotes openness and acceptance when errors are committed without fostering a nonchalant attitude. The Working Group recommends:

\section{Recommendation 9.1: Management should foster a culture in which it is understood that some human error is inevitable and that openness about errors leads to improvements in practice.}

\section{Operational Processes}

Operational processes refer to formal processes (e.g., operational tempo, time pressures, production quotas, incentive systems, and schedules), procedures (e.g., performance standards, objectives, documentation, and instructions about procedures), and oversight within the agency (e.g., organizational self-study, risk management, and establishment and implementation of a QA/QC process). Poor upper-level management decisions concerning each of these factors can also have a negative effect on the examiners' performance. Operational process factors include accreditation; appropriate standards, policies, or guidelines; documentation for standard operational procedures; overextension of resources; procedures that are not integrated into the training process; and, again, work and production schedules that produce risky decisions.

\section{Recommendation 9.2: Management should employ a system to identify and track errors and their causes.}

Appendix A gives a sample taxonomy of human factors issues and errors, including those discussed above. Appendix B includes an example of a Corrective Action Report, and Appendix $\mathrm{C}$ shows how the human factors described in the chapter apply to that particular error.

\subsection{Accreditation as a Means to Quality Improvement}

Accreditation is a multi-faceted process that ensures that the forensic laboratories follow a documented quality management system and adhere to standards of operation promulgated through standard-developing organizations. An accrediting body's routine onsite surveillance visits, required annual self-audit reports, and an externally monitored proficiency testing program assures periodic monitoring of the unit's compliance with the accreditation program's requirements.

Through accreditation, a forensic service provider demonstrates compliance with nationally and internationally recognized standards. Accreditation also ensures that the forensic unit has procedures in place to take appropriate corrective actions when indications of a significant problem appear. Unfortunately, there is a lack of uniformity in the application of existing accreditation standards in the United States, and different accrediting organizations apply different requirements (see Chapter 8). 


\subsubsection{Personnel Requirements}

Personnel requirements for accreditation include minimum standards for education, training, competency testing, and ongoing proficiency testing. Before assuming casework responsibility, each examiner should complete an established training program and should be tested for competency, at least in the most relevant work areas. Examiners should be routinely reviewed through annual proficiency testing, technical review of reports and examination records, and annual courtroom testimony monitoring (see Recommendation 6.4). Ongoing professional development for each examiner is an important element of the accreditation process.

Under the International Organization for Standardization's international standard ISO/IEC 17025, a forensic unit "must have arrangements to ensure that its management and personnel are free from any undue internal and external commercial, financial, and other pressures and influences that may adversely affect the quality of their work." ${ }^{590}$

\section{Box 9.3: Accreditation in the European Union}

In late November 2009, the European Union (EU) approved a measure known as the Framework Decision to standardize forensic laboratory accreditation in member states. Because of the high likelihood for cross-border crimes in the EU, judicial systems in each country must have confidence in laboratory results from other countries.

The standards require national accreditation bodies in each member state to grant accreditation to forensic laboratories that comply with ISO/IEC 17025, General requirements for the competence of testing and calibration laboratories. The accreditation standard specifically applies to laboratories that produce DNA profiles or work with latent print data. Each member state is responsible for ensuring compliance, for granting accreditation to their own laboratories, and for providing any funding necessary to achieve accreditation. The EU requires all latent print labs to comply with ISO/IEC 17025 by November 30, 2015. ${ }^{589}$

\subsubsection{Management Procedures}

Accreditation requires documented quality management procedures for the operation of the unit. The management system must designate a quality manager responsible for ensuring that the unit operates in conformity with the quality management system. Procedures must include steps to ensure that evidence is protected from loss, cross-transfer, contamination, or deleterious change. The procedures must ensure that evidence is properly marked and tracked throughout the period the unit controls the evidence.

\subsubsection{Technical Procedures}

Technical procedures must be documented, validated, and accepted in the scientific community. An accrediting agency conducts onsite assessments to ensure that the unit follows its documented procedures, adheres to the required standards, and uses proper controls.

\footnotetext{
${ }^{589}$ Council Framework Decision 2009/905/JHA of 30 November 2009 on Accreditation of Forensic Service Providers Carrying out Laboratory Activities. "Acts Adopted Under the Title VI of the EU Treaty." Official Journal of the European Union, 52, (December 9, 2009): 14-16.

${ }^{590}$ International Organization for Standardization. General Requirements for the Competence of Testing and Calibration Laboratories, ISO/IEC 17025:2005(E). 2nd ed. International Organization for Standardization, 2005.
}

183 Latent Print Examination and Human Factors: Improving the Practice through a Systems Approach The Report of the Expert Working Group on Human Factors in Latent Print Analysis 


\subsubsection{Facility Safety and Security}

The forensic unit should have a well-designed, efficient laboratory with adequate ventilation and safety equipment. All forensic units should follow applicable Occupational Safety and Health Administration standards, state and local health agency guidelines, and other safety regulations and should offer a mandatory safety training program (see Chapter 7).

The facility must have security to limit access to evidence, whether stored or under examination, and access to authorized individuals. The accreditation process evaluates the physical plant to ensure these standards are met.

\section{Recommendation 9.3: All forensic service providers should be accredited by a recognized accrediting body.}

\subsection{Case Review, Conflict Resolution, and Corrective Action}

Assuring the quality of forensic work, particularly in those disciplines in which the examiner is the sole provider of the results, can be challenging. Examiners can be influenced by a range of factors, including experience, fatigue, and stress. Not only are no two examiners exactly alike, but also an individual examiner's performance level can vary throughout the day. Thus, case review is an integral component of quality management. ${ }^{591}$

Case review involves verification, technical review, and administrative review. During the Verification phase of the Analysis, Evaluation, Comparison, and Verification process, a second examiner re-examines the latent prints to confirm or refute the conclusion of the case examiner. During technical review, another expert reviews the case file and report "to ensure the validity of scientific results and conclusions." 592 During administrative review, an expert examines the case file "for consistency with laboratory policy and for editorial correctness." 593 If discrepancies arise, the laboratory must have policies and procedures in place to address them. ${ }^{594}$ Numerous discrepancies can arise, but the remainder of this section focuses on disagreements during Verification, methods to resolve conflicting results, and corrective action.

\subsubsection{Verification}

Agencies should have a clear policy indicating which results will undergo verification. The Scientific Working Group on Friction Ridge Analysis, Study and Technology (SWGFAST) quality assurance guidelines state that source attributions "shall be verified prior to reporting"

\footnotetext{
${ }^{591}$ Scientific Working Group on Friction Ridge Analysis, Study and Technology. Quality Assurance Guidelines for Latent Print Examiners. Version 3.0. Scientific Working Group on Friction Ridge Analysis, Study and Technology, September 2006.

${ }^{592}$ American Society of Crime Laboratory Directors/Laboratory Accreditation Board. ASCLD/LAB-International Supplemental Requirements for the Accreditation of Forensic Science Testing Laboratories 2011 Edition. American Society of Crime Laboratory Directors/Laboratory Accreditation Board, approved September 11, 2010.

${ }^{593}$ Ibid.

${ }^{594}$ SWGFAST, 2006, op. cit.
} 
but that "exclusion or inconclusive results may be verified." 595 Verifying all results would be ideal (and some agencies do so). To conserve resources, however, agencies may verify all identifications but only a portion of the exclusions and inconclusive results.

The verifier typically receives the latent prints, the exemplar prints, the conclusions of the case examiner, and other information. In a blind verification, however, the verifier does not know the outcome of the first examination (see Chapters 1, 3, and 5). For blind verification to be meaningfully conducted, a sufficient proportion of all case conclusions must be reviewed. SWGFAST has released a Standard for the Application of Blind Verification of Friction Ridge Examinations ${ }^{596}$ to assist agencies instituting blind verification. ${ }^{597}$ Additional research into the costs and benefits of blind verification would be valuable.

\subsubsection{Conflict Resolution}

Once the agency establishes procedures for verifications, it also must adopt procedures for handling and documenting conflicting decisions. Conflict can occur because examiners have varying degrees of knowledge, skills, training, and experience. The agency must have a method to determine the result that will be reported as correct. Some agencies have a technical leader who makes the final decision. Others use a panel of examiners. Still others send conflicting results to another forensic service provider, which helps to mitigate some internal concerns (e.g., systemic error, personality conflicts, and bias) but is not practical for all agencies. Regardless of the procedure implemented by the agency, the existence of the disagreement should be noted in the report, and the basis for the final consensus should be stated in the report or other documentation. (For additional discussion of conflict resolution and how it differs from consultation, see Chapter 5, section 5.3.5.)

\subsubsection{Corrective Action}

Once the conflict has been resolved, an "error" will have to be attributed to either the case examiner or the verifier, and corrective action may be appropriate. ${ }^{598}$ For instance, failing to identify a single latent print on an item when the subject has been identified as leaving other prints on the item may not be critical. An occasional non-critical failure to identify does not necessitate a formal corrective action; however, routine failures to identify should result in a formal corrective action, as it may indicate a contributing issue such as training, medical issues, or the work environment. Regardless of any formal corrective actions, management should track errors to determine if there are chronic problems.

\footnotetext{
${ }^{595}$ Ibid. (emphasis added).

${ }^{596}$ Scientific Working Group on Friction Ridge Analysis, Study and Technology. Standard for the Application of Blind Verification of Friction Ridge Examinations. Version 1.0. Scientific Working Group on Friction Ridge Analysis, Study and Technology, February 2011.

${ }^{597}$ For discussion of a mixed system of blind and non-blind verification, see U.S. Department of Justice, Office of the Inspector General. A Review of the FBI's Progress in Responding to the Recommendations in the Office of the Inspector General Report on the Fingerprint Misidentification in the Brandon Mayfield Case. U.S. Department of Justice, 2011.

${ }^{598}$ SWGFAST, 2006, op. cit.
} 
The response of management to an error must be commensurate with the potential impact and frequency of the error. ${ }^{599}$ When errors occur, resolution may involve a simple consultation between the examiners or a formal corrective action. ISO/IEC 17025 accreditation standards state that (1) a laboratory must have policies and procedures regarding the implementation of corrective action, (2) the procedures must include an investigation to determine root causes, and (3) the laboratory must take preventive measures to mitigate the problem. ${ }^{600}$ Appendix B provides a sample Corrective Action Report, which describes the circumstances of an error, the corrective action, and the preventive measures.

Appendix $\mathrm{C}$ lists the human factors issues from all levels (examiner, conditional, supervisory, organizational) that contributed to the error in Appendix B. By compiling statistics on the various human factors issues related to each error, evaluating the data from multiple errors, and looking for common themes, management can become aware of critical failures in the system that could be setting the examiners up for failure. This systems approach could improve examiner performance not only within an agency but also throughout the profession.

\section{Recommendation 9.4: Management should establish policies and procedures for case review and conflict resolution, corrective action, and preventive measures.}

\subsection{Competency, Certification, and Proficiency Testing}

Certification and testing for competency and proficiency are components of a strong quality management system. Testing is one way to measure the examiner's knowledge, skills, and abilities. It can be used to demonstrate the examiner's minimum competency, achievement of proficiency, and continued proficiency. A competency test evaluates "a person's knowledge and ability prior to performing independent casework." 601 A proficiency test evaluates "the capability and performance of analysts, technical support personnel, and the laboratory; in open tests, the analysts and technical support personnel are aware that they are being tested; in blind tests, they are unaware. $" 602$

Many professions (e.g., language training, medicine, accountancy, and law) use testing and certification to demonstrate and acknowledge that an individual has met established minimum standards of skills and knowledge. In these professions, the testing process is typically called competency testing, and the official acknowledgement is called certification. Various professions handle certification and proficiency testing in different ways.

\footnotetext{
599 Ibid.

${ }^{600}$ ISO/IEC 17025:2005(E), op. cit.

${ }^{601}$ ASCLD/LAB, op. cit.

${ }^{602}$ Ibid.
} 
The process of competency testing, certification, and proficiency testing in the latent print discipline is largely ad hoc by jurisdiction and agency. It may be difficult to gain consensus on what competency, certification, and proficiency mean, let alone how they will be attained or documented. Yet, accreditation standards require competency testing prior to performing casework and the successful completion of a proficiency test by each examiner annually.

\section{Recommendation 9.5: The latent print community should develop and implement a comprehensive testing program that includes competency testing, certification testing, and proficiency testing.}

A standardized national testing program would be very useful in achieving this goal. To implement such a program, however, major tasks would need to be accomplished. Research should evaluate current training programs to discern similarities, weaknesses, and effectiveness (see Chapter 8). Training standards and standardized training material, including practical exercises, should be written, and criteria for selecting qualified trainers should be developed. A standardized test should be written with input from specialists in test design and validation. This test (or others) could be part of the framework for comprehensive testing described below.

\subsubsection{Competency Training}

During the training program, the new examiner should work toward a minimum level of knowledge and skill. To show that this level has been achieved, the examiner should pass a standardized competency test. Because examiners typically perform additional agency-specific functions (e.g., automatic fingerprint identification system entry, latent print development, and latent print photography), the agency or a national body should develop competency tests tailored to the examiner's duties.

187 Latent Print Examination and Human Factors: Improving the Practice through a Systems Approach The Report of the Expert Working Group on Human Factors in Latent Print Analysis

\section{Box 9.4: Certification and Testing for the Practice of Medicine}

Upon graduation from an accredited medical school, all medical school graduates are required to pass a national licensing examination before practicing medicine. The test is sponsored by the Federation of State Medical Boards and the National Board of Medical Examiners. The first part (on anatomy, physiology, biochemistry, pharmacology, pathology, microbiology, and behavioral sciences) typically is taken at the end of the second year of medical school. The second part (on clinical knowledge), includes demonstrations of clinical skills via simulated patient encounters and typically is taken in the fourth year of medical school. The final part is taken at the end of the student's formal medical school training and before internship and residency. This 2-day test measures the student's knowledge of basic science and medicine and assesses his or her ability to manage patient care via

After completing formal medical education at an accredited medical university and passing the competency examinations, U.S. medical students are required to complete a 1-year internship. This internship is typically part of the student's postgraduate residency in a chosen specialty (e.g., orthopedics, radiology, family practice). The internship is the minimum training requirement for a license to practice medicine in the U.S. and for taking most state medical board certification tests. All state medical specialties require periodic proof of continued professional competency. Continued professional competency often includes continuing medical education, recertification examinations, and some measure of clinical skills as approved by the certification board. 
After succeeding in the competency tests, an examiner can perform casework. A certified examiner should review the new examiner's casework at this stage. All conclusions should be verified, and each case should undergo technical review. This verification and technical review should continue until the agency determines that the examiner can work successfully under the agency's routine verification and technical review process. The examiner should not perform verification or technical reviews before becoming certified.

\subsubsection{Certification Testing}

A standardized certification test demonstrates that the examiner has become proficient in casework and meets or exceeds minimum competency to perform latent print examinations. To retain certification, an examiner should complete continuing education requirements and demonstrate proficiency in periodic proficiency tests.

\subsubsection{Proficiency Testing}

Periodic proficiency tests are a means to ensure that examiners maintain their skills. Agencies should administer proficiency tests at least annually that reflect the type of casework performed by the examiner. A standardized proficiency test for each examiner not only could demonstrate the necessary ability to continue as an examiner, but it also could inform training needs across the discipline. For instance, a high erroneous exclusion rate on latent palm prints that lack a clear distal orientation and anatomical region could indicate that training programs need to place more emphasis on palm prints or that examiners need periodic refresher training on palm prints. ${ }^{603}$

\subsection{Summary}

This chapter offers recommendations about the principles, policies, and practices of management and describes strategies to prevent human errors in latent print examinations. Management within any forensic operation must be committed to developing, maintaining, and improving quality assurance and quality control. Latent print units should report accurate and timely results. The examiners have the responsibility to provide results and testimony based upon scientific or technical knowledge, training, and experience. Management must create the proper environment for this to occur. This includes creating an impeccable QA/QC process; meeting standards of certification, accreditation, and proficiency testing; and adopting a systems approach to reducing errors.

${ }^{603}$ Proficiency testing for other purposes, such as estimating error rates, is considered in Chapter 2. 


\section{Appendix 9-A: Sample Taxonomy of Errors and Human Factors}

The following tables contain a classification scheme that outlines potential sources of error. Additional factors at every level could always occur and should be documented and tracked.

\section{Examiner Actions}

\begin{tabular}{|l|}
\hline \multicolumn{1}{|c|}{ Decision-Based Mistakes } \\
\hline Conclusion exceeded ability of examiner \\
\hline Failure to recognize exemplars as inadequate \\
\hline Failure to search all exemplars \\
\hline Failure to use available technology \\
\hline Hurried or insufficiently thorough examination \\
\hline Improper anatomical source of data \\
\hline Improper orientation of images \\
\hline Incomplete search \\
\hline Insufficient data to support conclusion \\
\hline Misprioritized level of effort \\
\hline Poor target group selection \\
\hline Print improperly deemed suitable \\
\hline Print improperly deemed unsuitable \\
\hline
\end{tabular}

\begin{tabular}{|l|}
\hline \multicolumn{1}{|c|}{ Perception-Based Mistakes } \\
\hline Not all data perceived \\
\hline Data perceived and compared inadequately \\
\hline
\end{tabular}

\begin{tabular}{|l|}
\hline \multicolumn{1}{|c|}{ Skill-Based Mistakes } \\
\hline Failure to find target data \\
\hline $\begin{array}{l}\text { Failure to properly weight the significance of the } \\
\text { data }\end{array}$ \\
\hline Failure to recognize distortion \\
\hline Inability to reach a conclusion \\
\hline Incomplete comparison \\
\hline Misinterpreted data \\
\hline Not all data understood \\
\hline Poor search technique \\
\hline
\end{tabular}

\begin{tabular}{|l|}
\hline \multicolumn{1}{|c|}{ Examiner Violations } \\
\hline Deeming a latent print not suitable intentionally \\
\hline Disregarding QA/QC procedures (e.g., verification) \\
\hline Disregarding discrepant information \\
\hline Erroneously identifying a latent print (intentionally) \\
\hline Identifying an unsuitable latent print (intentionally) \\
\hline Including a subject inappropriately (intentionally) \\
\hline Misrepresentation of information \\
\hline $\begin{array}{l}\text { Not comparing a latent print and reporting exclusion } \\
\text { or inconclusive results }\end{array}$ \\
\hline $\begin{array}{l}\text { Seeking/coercing another examiner to verify a } \\
\text { complex comparison }\end{array}$ \\
\hline
\end{tabular}




\section{Conditions that Affect Performance}

\begin{tabular}{|l|}
\hline \multicolumn{1}{|c|}{ Adverse Mental States of the Examiner } \\
\hline Anger \\
\hline Apprehension to reach a conclusion \\
\hline Bias \\
\hline Boredom \\
\hline Channelized attention \\
\hline Complacency \\
\hline Compromised integrity or ethics \\
\hline Distraction \\
\hline Expectancy \\
\hline Frustration \\
\hline Haste \\
\hline Lack of confidence \\
\hline Mental fatigue \\
\hline Misplaced motivation \\
\hline Overconfidence \\
\hline Peer pressure \\
\hline Preoccupation with personal problems \\
\hline Task overload \\
\hline Stress \\
\hline
\end{tabular}

\section{Physical or Mental Limitations of the Examiner}

Chronic psychological disorder

Color vision deficiency

Dyslexia

Inadequate training

Incompatible intelligence/aptitude

Limited experience

Visual limitations (e.g., acuity or contrast sensitivity)

\begin{tabular}{|l|}
\hline \multicolumn{1}{|c|}{ Communication } \\
\hline Confusing or conflicting directions or demands \\
\hline Failure to convey adequate information \\
\hline Failure to use all available sources of information \\
\hline Inadequate communication among examiners \\
\hline Inadequate communication with management \\
\hline Inadequate report writing skills \\
\hline Inadequate teamwork \\
\hline Inadequate case documentation \\
\hline Standard terminology not used \\
\hline
\end{tabular}

\section{Adverse Physiological States of the Examiner}

Alcohol use (intoxicated or hung-over)

Eyestrain

Illicit drug use

Inadequate nutrition (poor dietary practices)

Lack of sleep

Medical illness

Physical fatigue (e.g., postural fatigue)

Physical injuries

Medication side effects

\begin{tabular}{|l|}
\hline \multicolumn{1}{|c|}{ Physical Environment } \\
\hline Clutter \\
\hline Excessive heat or cold \\
\hline Inadequate lighting \\
\hline Inadequate ventilation \\
\hline Poor workplace design \\
\hline Noise interference \\
\hline
\end{tabular}

\begin{tabular}{|l|}
\hline \multicolumn{1}{|c|}{ Technical Environment } \\
\hline Defective equipment \\
\hline Defective tools \\
\hline Inadequate/outdated software or equipment \\
\hline
\end{tabular}




\section{Supervisory Issues}

\begin{tabular}{|l|}
\hline \multicolumn{1}{|c|}{ Leadership Failures } \\
\hline Change induced without training \\
\hline $\begin{array}{l}\text { Failure to provide professional guidance and } \\
\text { oversight }\end{array}$ \\
\hline Failure to set proper example \\
\hline Failure to track job qualifications or skills \\
\hline Inadequate assessment of required skill \\
\hline Inadequate leadership job knowledge \\
\hline Inadequate monitoring of work \\
\hline Inadequate or incorrect performance feedback \\
\hline Inadequate performance measurement or evaluation \\
\hline Insufficient initial or ongoing training provided \\
\hline Inadequate or inappropriate incentives \\
\hline Inadequate coaching or mentoring on skill \\
\hline Inadequate measurement of training effectiveness \\
\hline Personality conflicts \\
\hline Unrealistic expectations \\
\hline
\end{tabular}

\begin{tabular}{|l|}
\hline \multicolumn{1}{|c|}{ Operational Planning Failures } \\
\hline Excessive workload \\
\hline Failure to provide adequate rest breaks \\
\hline Improper or insufficient delegation of work \\
\hline $\begin{array}{l}\text { Inadequate communication of policy, procedure, } \\
\text { practices, or guidelines }\end{array}$ \\
\hline Inadequate documentation \\
\hline $\begin{array}{l}\text { Inadequate matching of individual qualifications and } \\
\text { job or task requirements }\end{array}$ \\
\hline Setting objectives, goals, or standards that conflict \\
\hline Unclear or conflicting assignment of responsibility \\
\hline Unrealistic deadlines or quotas \\
\hline
\end{tabular}

\begin{tabular}{|l|}
\hline \multicolumn{1}{|c|}{ Failure to Correct Known Problems } \\
\hline Failure to correct inappropriate behavior \\
\hline Failure to correct reported problem \\
\hline Failure to correct workplace hazards \\
\hline Failure to initiate corrective action \\
\hline Failure to update standard operating procedures \\
\hline Rewarding or tolerating improper performance \\
\hline
\end{tabular}

\begin{tabular}{|l|}
\hline \multicolumn{1}{|c|}{ Supervisory Violations } \\
\hline Enabling excessive risk-taking \\
\hline Failure to enforce rules and regulations \\
\hline Supervisor encouragement of rule bending \\
\hline Fraudulent documentation \\
\hline Management-induced haste \\
\hline Unrealistic production goals \\
\hline Undue influence toward a desired outcome \\
\hline $\begin{array}{l}\text { Violation of standard operating procedures by } \\
\text { supervisor }\end{array}$ \\
\hline
\end{tabular}




\section{Organizational Influences}

\begin{tabular}{|l|}
\hline \multicolumn{1}{|c|}{ Resource Management Failures } \\
\hline Failure to correct known design flaws \\
\hline Inadequate evaluation and promotion policies \\
\hline Inadequate hiring, firing, and promotion processes \\
\hline Inadequate matching of qualifications for the job \\
\hline Ineffective cost-cutting \\
\hline Lack of logistical support \\
\hline Purchasing unsuitable equipment/parts \\
\hline Inadequate selection of personnel \\
\hline Inadequate staffing \\
\hline Use of inadequate contractor or vendor \\
\hline Unfunded directives \\
\hline
\end{tabular}

\begin{tabular}{|l|}
\hline \multicolumn{1}{|c|}{ Organizational Climate Failures } \\
\hline $\begin{array}{l}\text { Inadequate accessibility or visibility of upper } \\
\text { management }\end{array}$ \\
\hline Inadequate allocation of resources \\
\hline Inadequate formal accountability for actions \\
\hline Dysfunctional organizational culture \\
\hline $\begin{array}{l}\text { Organizational values not clearly defined and } \\
\text { articulated }\end{array}$ \\
\hline Unclear or conflicting assignments of responsibility \\
\hline
\end{tabular}

\begin{tabular}{|l|}
\hline \multicolumn{1}{|c|}{ Operational Process Failures } \\
\hline Failure to maintain accreditation \\
\hline Lack of appropriate standards, policies, or guidelines \\
\hline $\begin{array}{l}\text { Inadequate documentation for standard operating } \\
\text { procedures }\end{array}$ \\
\hline Organizationally induced time pressure \\
\hline Overextending resources \\
\hline Procedures not integrated into training process \\
\hline $\begin{array}{l}\text { Quality process not adequately implemented or } \\
\text { maintained }\end{array}$ \\
\hline Unrealistic quotas established by the organization \\
\hline $\begin{array}{l}\text { Work or production schedules that produce risky } \\
\text { decisions }\end{array}$ \\
\hline
\end{tabular}




\section{Appendix 9-B: Corrective Action Report Sample}

FORENSIC LAB CORRECTIVE ACTION REPORT

\begin{tabular}{|c|c|}
\hline \multicolumn{2}{|c|}{ Place "N/A" in blanks that are not applicable. } \\
\hline APPLICABLE \#(S) (e.g., Event \#, CTS \#) & RELEVANT INSTRUMENT: \\
\hline \multirow[t]{4}{*}{ Case: $12356-78$} & Instrument type: N/A \\
\hline & Manufacturer: N/A \\
\hline & Model \#: N/A \\
\hline & Serial \#: N/A \\
\hline \multicolumn{2}{|c|}{ DESCRIPTION OF INCIDENT/EFFECT OF DISCREPANCY } \\
\hline \multicolumn{2}{|c|}{$\begin{array}{l}\text { Forensic scientist (FS) Jane Doe was assigned homicide case \#123456-78. FS Doe worked the case } \\
\text { during November and early December } 2009 \text {. FS Doe completed the initial formal report in December } \\
2009 \text {. She developed latent prints on } 4 \text { items of evidence and recovered } 16 \text { latent prints. She deemed } \\
\text { two of these suitable for comparison. }\end{array}$} \\
\hline \multicolumn{2}{|c|}{$\begin{array}{l}\text { FS Doe also examined } 3 \text { latent print packets submitted by Crime Scene Examiners (CSA), collectively } \\
\text { containing } 56 \text { lifts/photos. On these lifts/photos, } 75 \text { suitable latent prints were present. }\end{array}$} \\
\hline \multicolumn{2}{|c|}{$\begin{array}{l}\text { FS Doe compared the } 77 \text { suitable latent prints to those of } 5 \text { suspects, } 1 \text { victim, and } 1 \text { other subject } \\
\text { identified via the automated fingerprint identification system (AFIS). This amounts to } 539 \text { conclusions. } \\
\text { One suspect was identified to one of the latent prints recovered from the evidence. Another suspect } \\
\text { was identified to one latent print submitted by CSA. The AFIS subject was identified to } 28 \text { latent prints } \\
\text { submitted by the CSA. }\end{array}$} \\
\hline \multicolumn{2}{|c|}{$\begin{array}{l}\text { Manager Susan Smith verified these results on February 4, 2010. Manager Smith noted that two of the } \\
\text { identifications recorded by FS Doe were to the incorrect finger of the correct subject (AFIS subject). FS } \\
\text { Doe originally identified the two latent prints to the right thumb of the subject; however, one was made } \\
\text { by the right middle finger, and the other was made by the right ring finger of the same subject. }\end{array}$} \\
\hline \multicolumn{2}{|c|}{$\begin{array}{l}\text { Manager Smith noted the correct conclusion in the case notes and asked FS Doe to review her conclu- } \\
\text { sions. FS Doe immediately noticed the errors. Manager Smith verbally notified the quality assurance } \\
\text { (QA) manager and the laboratory director on February } 8,2010 \text {. The case was returned to FS Doe, and } \\
\text { she created charted enlargements for her case file and annotated the notes appropriately. Manager Smith } \\
\text { completed a technical review of the case on February } 25,2010 \text {, and issued the report. }\end{array}$} \\
\hline \multicolumn{2}{|c|}{ There was no effect of the discrepancy, as the errors were caught during the Verification phase. } \\
\hline Date: $3 / 4 / 11$ & eu by. IVIallagel SIIILII \\
\hline
\end{tabular}




\section{ROOT CAUSE DETERMINATION}

The right thumb, middle, and ring fingers of the subject had similar ridge configurations in the core. The similar pattern, ridge count, and ridge configuration in these fingers likely contributed to the errors.

In addition, FS Doe was under significant stress during this time period because of the terminal illness of a parent.

FS Doe also indicated to Manager Smith that she was trying to increase her case productivity to help alleviate the backlog. The pressure FS Doe was placing upon herself to complete the case may also have contributed to the error.

FS Doe was increasing the use of fingerprint loupes into the analysis of her casework during this time period to prepare for the International Association of Identification (IAI) Latent Print Certification Test. This was a significant departure from her normal routine, which focuses on the use of digital imaging during her comparisons. (The IAI certification test does not permit use of digital imaging technology.) FS Doe normally charts her identifications via Photoshop, but she did not do so in this case because she used the loupes.

The cumulative effect of the stress of life events, similarity of the prints, time pressure, and technology adaptations ultimately resulted in these errors.

Determined By: Manager Smith

\begin{tabular}{|c|c|}
\hline \\
\hline \multicolumn{2}{|c|}{$\begin{array}{l}\text { Corrective Action } \\
\text { FS Doe was placed on supervised casework for a period of } 30 \text { days. Nine cases were fully reviewed } \\
\text { and re-examined during this time. No errors were noted. Upon completion of the } 30 \text { days of supervised } \\
\text { casework, FS Doe was assigned a Collaborative Testing Services test as in-house proficiency test on } \\
\text { March 17, 2011. FS Doe successfully completed this test on April 1, 2011. }\end{array}$} \\
\hline $\begin{array}{l}\text { From November } \\
\text { reviewed. No en } \\
\text { reviewed during } \\
\text { recovered. }\end{array}$ & $\begin{array}{l}\text { 1, FS Doe completed ten cases. Seven of these cases were } \\
\text { fications in these cases. The three cases that were not } \\
\text { evelopment cases, and no comparable latent prints were }\end{array}$ \\
\hline \multicolumn{2}{|c|}{$\begin{array}{l}\text { Preventive Measures } \\
\text { FS Doe and Manager Smith analyzed and marked } 30 \text { difficult latent prints to evaluate FS Doe's ability } \\
\text { to interpret and detect minutiae. Once marked, FS Doe and Manager Smith compared the markings and } \\
\text { discussed the results for each image. The goal of this exercise was to ensure that FS Doe was correctly } \\
\text { assessing friction ridge detail. FS Doe successfully completed this exercise: Manager Smith did not } \\
\text { note any problems with FS Doe's interpretation of the friction ridge detail. }\end{array}$} \\
\hline $\begin{array}{l}\text { Forensic } \mathrm{L} \\
\text { hes. Over tim } \\
\text { amination to }\end{array}$ & $\begin{array}{l}\text { dd and work product more carefully during high-stress } \\
\text { are not mentally able to perform the task and postpone the } \\
\text { hould be re-assigned in this situation. }\end{array}$ \\
\hline \multicolumn{2}{|c|}{$\begin{array}{l}\text { If FS Doe chooses to use the fingerprint loupe in casework, the comparison will be repeated utilizing } \\
\text { digital imaging to determine if the re-examination yields any inconsistencies. }\end{array}$} \\
\hline ate: $05 / 3 / 2011$ & Approved By: QA Manager \\
\hline
\end{tabular}




\begin{tabular}{||l|l|c||}
\hline ROUTE TO IN LAB: (NAME \& P \#) & \multicolumn{1}{|c||}{ SIGNATURE } & \\
\hline FS Jane Doe & & \\
\hline Manager Smith & & \\
\hline QA Manager & & \\
\hline Laboratory Director ADDITIONAL NOTIFICATIONS, IF REQUIRED: \\
\hline \hline \multicolumn{2}{|c||}{ DATE NOTIFIED } \\
\hline PERSON NOTIFIED: (NAME \& TITLE / P \#) & \\
\hline \multicolumn{2}{|c|}{} \\
\hline ASCLD/LAB Notified: $\square$ Yes $X$ Not Required & \\
\hline \hline
\end{tabular}

\section{NOTES:}

Jane Doe has not made any erroneous identifications since this incident - QA Manager 08/26/20101

THIS SECTION WILL BE COMLPETED BY THE QUALITY ASSURANCE MANAGER

Was the problem corrected? $\times$ Yes $\square$ No (if no, complete section below) FOLLOW-UP, IF REQUIRED:

N/A - QA Manager 


\section{Appendix 9-C: Applying Human Factors to an Error}

Human factors issues have an impact on examiner performance. The error described in the Corrective Action Report (CAR) in Appendix B was the culmination of a breakdown in the system, not just the fault of the examiner. The examiner involved is a dedicated professional and an excellent casework examiner. In reviewing the CAR, one can look at the various holes in the Swiss cheese model at each level: Examiner Actions, Conditions that Affect Performance, Supervisory Issues, and Organizational Influences.

\section{Examiner Action: Decision-Based Mistakes}

Failure to use available technology

Hurried or insufficiently thorough examination

Misprioritized level of effort

\section{Examiner Action: Skill-Based Mistake}

Misinterpreted data

\section{Examiner Action: Perception-Based Mistake}

Not all data perceived

\section{Conditions: Adverse Mental States}

Preoccupation with personal problems

Stress

\begin{tabular}{|l|}
\hline \multicolumn{1}{|c|}{ Supervisory Issues: Leadership Failures } \\
\hline Inadequate assessment of required skill \\
\hline Inadequate monitoring of work \\
\hline Inadequate coaching or mentoring on skill \\
\hline
\end{tabular}

Organizational: Resource Management Failures

Inadequate staffing

Organizational: Operational Process Failures

Organizationally induced time pressure

Overextending resources 


\section{Chapter 10: Summary of Recommendations}

The recommendations and suggestions of the Working Group address issues ranging from the acquisition of impressions of friction ridge skin to courtroom testimony, from laboratory design and equipment to research into emerging methods for associating latent prints with exemplars. This group is not the first to address most of these matters; the recommendations are informed by and build upon the work of many practitioners and scholars of forensic science and evidence as well as the experience of other industries and enterprises that must deliver safe products or services. This concluding chapter collects and organizes the recommendations put forth on the following topics:

- Performing Analysis, Comparison, Evaluation, and Verification (ACE-V)

- Reporting and testifying

- Managing the process

- Improving training and education

- Providing facilities and equipment

- Supporting research efforts.

\subsection{Performing Analysis, Comparison, Evaluation, and Verification (ACE-V)}

Latent print examinations can produce very powerful evidence regarding the possible association between a suspect and the source of a print. The long-established practice in latent print examinations is to interpret the evidence in terms of the categorical conclusion that two prints do or do not share a common source (or to report that the information presented does not permit such a categorical conclusion). Traditionally, a fingerprint identification was considered an "individualization," meaning that the latent print was considered identified to one finger of a specific individual to exclude every other potential source in the universe. However, this claim is needlessly strong, not yet adequately supported by fundamental research, and impossible to validate solely on the basis of experience. Nor does fingerprint evidence have objective standards or a well-validated statistical model that can provide an objective measure of its strength in a given instance. Therefore, examiners should not claim to be able to exclude every other finger in the world as a potential source. Rather, an identification decision suggests a substantial enough similarity that, based on the examiner's training and experience, the examiner believes that the two impressions originated from a common source. Whether any other finger in the entire world might also be able to leave an impression with a comparable amount of similarity is not fully known, and the examiner's testimony should not suggest otherwise. Regardless of the specific words used to describe an identification, examiners should refrain from claiming that an identification means that they have excluded all other individuals in the world.

Because empirical evidence and statistical reasoning do not support a source attribution to the exclusion of all other individuals in the world, latent print examiners should not report or testify, directly or by implication, to a source attribution to the exclusion of all others in the world. (Recommendation 3.7) 
The alternatives to the theory of universal individualization include probabilistic forms of source attribution (see Chapter 1), qualitative or quantitative likelihood ratios (determined subjectively or, in the future, with probabilistic models, as discussed in Chapters 4 and 6), or a broader range of conclusions than the three choices of identification, exclusion, or inconclusive. Without taking a position on which of these approaches is best, the Working Group recommends:

The friction ridge examiner community and other experts should determine under what circumstances a qualified rather than an absolute conclusion is warranted. (Recommendation 3.8)

However the conclusion of a latent print examination might be framed, the examination process requires the careful interpretation and comparison of friction ridge impressions. From the first examination of the features contained in a latent print to a final conclusion regarding an identification or exclusion, an examiner engages in many acts of interpretation. Current methods for making these interpretations are based on professional knowledge and experience rather than on formal decision thresholds or statistical models. The process known as ACE-V organizes the interpretations and decisions of an examiner into a useful and logical sequence, but descriptions of this process do not detail the substantive content of the various steps. Examining latent prints and exemplars necessitates judgment and expertise, which inevitably makes the interpretive process partly subjective. With this subjectivity of interpretation comes the possibility of reduced performance due to a wide range of human factors issues. To increase the transparency of the process and to insulate the examiner from extraneous influences, the Working Group makes the following recommendations:

A report and contemporaneous supporting notes or materials should document the examination to make the interpretive process as transparent as possible. Although the degree of detail may vary depending on the perceived complexity of the comparison, documentation should, at a minimum, be sufficient to permit another examiner to assess the accuracy and validity of the initial examiner's assessment of the evidence. (Recommendation 3.1)

Modifications to the results of any stage of latent print analysis (e.g., feature selection, utility assessment, discrepancy interpretation) after seeing a known exemplar should be viewed with caution. Such modifications should be specifically documented as having occurred after comparison has begun. (Recommendation 3.2)

Procedures should be implemented to protect examiners from exposure to extraneous (domain-irrelevant) information in a case. (Recommendation 3.3)

Several steps in the latent print analysis process require a determination of latent print utility or sufficiency. The examiner must determine whether the print has enough clarity and quantity of information for some specified purpose. This happens both early in the process (e.g., determinations of whether the latent is suitable for analysis or suitable for an automated fingerprint identification system search) and later in the process (e.g., determinations of sufficient information to compare the latent and exemplar). Forensic service providers can enhance the transparency of their analyses and conclusions by promulgating clear guidelines that 
assist examiners in making determinations of utility. ${ }^{604}$ Therefore, the Working Group recommends:

Each agency or forensic service provider should define "suitable" or "sufficient" in its standard operations procedures. These guidelines should be as explicit as possible about what is expected for sufficiency determinations at different stages of the latent print examination process. (Recommendation 3.4)

Information that could be incorporated in an agency's guidelines includes, for example, number of minutiae and visibility of ridge flow, pores, ridge edges, and details of incipient ridges, creases, and scars. ${ }^{605}$ For a more detailed discussion of suitability, see Chapter 3, section 3.4.

Modern investigations often compare latent prints to exemplars from automated fingerprint identification system (AFIS) searches. When looking in a large database for those prints that bear the greatest similarity to the latent print in question, these programs could select a print from a non-source that is far more similar to the latent print than examiners are accustomed to seeing from non-matching sources. An examiner whose idea of the necessary amount of information for a match comes from a non-database world may not be taking into account the possibility of incidental similarity that a large database carries with it. Special care may be warranted in interpreting the evidence in such cases. Thus, the Working Group recommends:

When comparing latent prints to exemplars generated through AFIS searches, examiners must recognize the possibility and dangers of incidental similarity. Adjustments such as a higher decision threshold, stricter tolerances for differences in appearance, and explicit feature weighting need to be considered. Modified quality assurance practices for this scenario also should be considered. (Recommendation 3.6)

\subsection{Reporting and Testifying}

The forensic part of forensic science distinguishes it from other scientific disciplines. Outside the forensic arena, scientists must report their results, but they rarely do so to inform criminal investigators, prosecutors, defense counsel, and courts. The needs of these lay consumers of forensic science evidence lead to two recommendations. First:

The report of the examination should ensure that the findings and their limitations are intelligible to non-experts. (Recommendation 5.1)

Second, to maximize the likelihood that the evidence will be used properly, fully, and fairly at trial, the report of the results of an examination should:

\footnotetext{
${ }^{604}$ This refers to guidelines for deciding whether a print warrants further assessment and not to guidelines for source attribution. Thus, a rule that specifies a minimum number of minutiae for sufficiency might be reasonable because it creates a process that is consistent throughout the forensic service provider and transparent to others. Given current information, however, a similar rule would not be appropriate for ultimate decision making.

${ }^{605}$ The guidelines for determining whether a latent print is suitable for comparison are necessarily different than the guidelines for comparing a pair of prints. The Working Group takes no position on whether agencies should couch guidelines as recommendations or as requirements.
} 
a. Identify the latent print examiner(s);

b. Describe the items submitted to the examiner(s);

c. List the procedures used by the examiner to develop, visualize, or enhance the friction ridge impressions;

d. List all comparisons conducted;

e. State all conclusions with the method used to reach them;

f. Note any important limitations to the conclusions;

g. Indicate whether a verification was made and whether there was any conflict of opinion among examiners prior to the reported conclusions;

h. Note (or refer to external documentation of) any information about the case that the examiner(s) received;

i. Note the existence of additional documentation; and

j. Define important technical terms, either explicitly or by reference to an authoritative, readily available source. (Recommendation 5.2)

Latent print examiners do more than analyze impressions and prepare written records of their work. They testify as expert witnesses who have more latitude than ordinary fact witnesses to provide opinions and guidance to jurors. As expert witnesses and as forensic science professionals, they have an obligation to their profession and to the court to maintain "objectivity, staying within the bounds or limits of what the science can provide, maintaining confidentiality, complying [with] legal demands such as disclosure, and being truthful." ${ }^{606}$ To help maintain this objectivity and "to promote the understanding of examinations and findings," ${ }^{107}$ the Working Group recommends:

Forensic service providers should adopt codes of ethics that require testifying in a nonpartisan manner; answering questions from both the prosecution and the defense directly, accurately, and fully; and providing appropriate scientific information before, during, and after trial. (Recommendation 6.2)

The importance of "staying within the bounds or limits of what the science can provide" and providing appropriate scientific information means that, as previously noted in section 10.1, "latent print examiners should not report or testify, directly or by implication, to a source attribution to the exclusion of all others in the world" (see Recommendation 3.7).

These goals are most likely to be realized when the expert confers with the lawyer prior to direct examination. At a pretrial meeting, the expert can make clear the nature and limits of the testimony that the evidentiary material warrants and can work with the lawyer to present this information fully, fairly, and clearly.

The trial preparation process should address the presentation of technical information in lay terms, the organization of the direct examination, possible cross-examination, and the possible use of visual aids. (Recommendation 6.1)

\footnotetext{
${ }^{606}$ Budowle, B., M. Bottrell, S. Bunch, et al. "A Perspective on Errors, Bias, and Interpretation in the Forensic Sciences and Direction for Continuing Advancement.” Journal of Forensic Sciences, 54 (July 2009): 803.

${ }^{607}$ Scientific Working Group on Friction Ridge Analysis, Study and Technology. A Model Policy for Friction Ridge Examiner Professional Conduct. Version 1.0. Scientific Working Group on Friction Ridge Analysis, Study and Technology, December 2008.
} 
Because absolute certainty is unattainable in science, the witness must be prepared to acknowledge and discuss the possibility that an opinion is not correct. Therefore:

A testifying expert should be familiar with the literature related to error rates. A testifying expert should be prepared to describe the steps taken in the examination process to reduce the risk of observational and judgmental error. The expert should not state that errors are inherently impossible or that a method inherently has a zero error rate. (Recommendation $6.3)$

Finally, forensic service providers should maintain quality assurance mechanisms for reporting and testifying.

An agency that employs latent print examiners should establish requirements and guidelines for reporting, documentation, and testimony. The agency should review a sample of every examiner's case reports, documentation, and testimony and affidavits at least annually to verify compliance with these requirements and guidelines. (Recommendation 6.4)

\subsection{Managing the Process}

Latent print examinations do not occur in isolation. Supervision of the staff members and management of the facilities are essential to risk reduction and quality assurance and control. Effective management requires good information about the incidence and sources of errors. Making this information available requires a culture in which both management and staff understand that openness about errors is not necessarily a path to punitive sanctions but rather is part of an effective system to detect deviations from desired practices and incorrect judgments in latent print casework. To achieve such a system, the Working Group recommends:

Management should foster a culture in which it is understood that some human error is inevitable and that openness about errors leads to improvements in practice. (Recommendation 9.1)

Management should employ a system to identify and track errors and their causes. (Recommendation 9.2)

Management should establish policies and procedures for case review and conflict resolution, corrective action, and preventive measures. (Recommendation 9.4)

Furthermore, adherence to appropriate standards is vital to achieving high levels of performance. As a result:

All forensic service providers should be accredited by a recognized accrediting body. (Recommendation 9.3)

The latent print community should develop and implement a comprehensive testing program that includes competency testing, certification testing, and proficiency testing. (Recommendation 9.5) 
Forensic service providers should require personnel to become certified through an accredited program. Certifying bodies should review current certification tests with the aid of professionals in test design and psychometrics. (Recommendation 8.7 in part)

Finally, to ensure that latent print examiners maintain their physical capacity to perform at high levels of accuracy:

Forensic service providers should institute medical surveillance for examiners with respect to activities specific to friction ridge examination. Corrective measures should be taken when appropriate and recorded. The program should include a baseline visual examination followed by annual vision testing to detect vision deficiencies that may affect interpretation of the evidence. (Recommendation 7.2 in part)

\subsection{Improving Training and Education}

The need to train and educate examiners on existing procedures and equipment and on emerging technologies is a recurring theme in this report. The Working Group's recommendations involve university education, on-the-job training and mentoring, and continuing education. Specifically, the Working Group recommends:

Forensic service providers should require personnel to participate in continuing education. (Recommendation 8.7 in part)

The latent print examiner community should expand the training of examiners in elementary probability theory to enable examiners to properly utilize the output of probabilistic models. (Recommendation 4.3)

The federal government should consider funding research to determine what educational and cognitive abilities should be prerequisites for training a latent print examiner. Forensic service providers should have systematic training programs that include these abilities as well as education in the scientific method, reading and understanding relevant scientific literature, communication skills, and methods for logically developing conclusions. (Recommendation 8.1)

Agencies should develop formal mentoring programs that define the responsibilities of the mentor, trainee, and supervisor. (Recommendation 8.3)

Certifying bodies should ensure that examiners have been trained to competency. Laboratory accrediting bodies should evaluate the effectiveness of an agency's training program.

(Recommendation 8.8)

Because statistical information plays a fundamental role in weighting latent print feature evidence, training should include the best available empirical information and should educate examiners about probabilistic reasoning in using that information. (Recommendation 3.5) 
The federal government should establish an expert group to develop a latent print educational textbook, practical exercises, and assessment tests (print and online) in consultation with professional organizations, senior latent print examiners, researchers with content knowledge, experienced trainers, and professionals experienced in curriculum development. (Recommendation 8.4)

The federal government should support a clearinghouse of materials from existing training programs as well as publications on latent print identification. (Recommendation 8.6)

Training materials should include topics beyond the technical aspects of friction ridge analysis, such as:
a. Documentation of work and case notes and written and oral communication;
b. Professional ethics;
c. Human factors issues such as fatigue, bias, cognitive influences, perceptual influences, and error;
d. Research methods; and
e. Legal and ethical aspects of expert testimony. (Recommendation 8.5)

The federal government should develop a forensic latent print examiner scholarship program to:

a. Fund the post-hire training program and, upon successful completion, provide tuition reimbursement, loan forgiveness, or other financial assistance to degreed latent print examiner trainees working in state, local, or private (not-for-profit) forensic laboratories; and

b. Reimburse the employer for a percentage of the salaries during the training period. (Recommendation 8.2)

\subsection{Providing Facilities and Equipment}

Latent print examinations take place in a physical environment with hardware and software that can facilitate the work of the examiners. To achieve the best results in examinations, the Working Group recommends:

A structured approach that involves users throughout the entire design and implementation process should be followed when designing technology systems and the physical work environment. In addition, forensic service providers should perform usability testing to optimize user performance and user satisfaction before deployment. (Recommendation 7.1)

\subsection{Supporting Research Efforts}

An increasing number of research projects are underway, but additional research should be undertaken in many areas. For example, the Working Group encountered many questions about the existing interpretive process, possible enhancements to it, and other significant matters that presently lack definitive answers. There is a critical need for a focused program of research into the interpretive process that is at the heart of ACE-V. For example, only a handful of studies have assessed variation in the feature selection process. Generally, the findings have shown wide variation among examiners during the task of feature selection. Developing methods to reduce 
the variation of the print features selected for comparisons and the development of tools and technologies to assist in the identification of the most reliable features would both be useful. While these tools are likely to be especially important for complex comparisons, they may have broader benefits. Standardized methods for feature selection should result in less variation in the features that are selected and compared, which in turn should lead to more consistency in the decisions reported. However, even if decisions were more consistent, they would not necessarily be more accurate. Even the best interpreters of prints should be willing to compare their own methods to a standard protocol to decide which practice is best.

Furthermore, the link between variations in feature selection and the examiner's ultimate decision is not well understood. Emerging research suggests that there is a relationship, but the effect may be competing with other major interpretative steps, such as interpreting discrepancy, feature weighting, and thresholds for reaching identification decisions. A better understanding of the link between feature selection and the ultimate decision would permit best practices to be formulated. This knowledge would also allow technology systems to select the most reliable and useful features on which to base decisions later in the ACE-V process.

The Working Group found no research that explicitly addresses utility or sufficiency in the context of latent print analysis. This is unsurprising, for a critical piece for any such researchthe definition and validation of a metric for assessing utility - is missing. Research has been carried out related to the examiner decision process that implicitly involves judgments of utility and sufficiency, but these studies tend to focus only on the accuracy or repeatability of the ultimate decision. For example, the Noblis-FBI study described in Chapter 2 treats the ACE-V process as a "black box." The experimenters provide the input and measure the output in various ways. This kind of research provides valuable information on how examiners perform relative to "ground truth" (accuracy) and how their results compare to one another (reliability). Opening the box to study the process of judgment in every phase of ACE-V would provide the empirical foundation from which to develop best practices for each part of the process. As a result, the Working Group recommends:

The federal government should support a research program that aims to:

a. Develop measures and metrics relevant to the analysis of latent prints;

b. Use such metrics to assess the reproducibility, reliability, and validity of various interpretive stages of latent print analysis; and

c. Identify key factors related to variations in performance of latent print examiners during the interpretation process. (Recommendation 3.9)

Practitioners recognize that assigning weights to latent print features and their configurations is a fundamental decision-making or interpretive step, either as an output of the Analysis phase or as part of the Evaluation phase. Pioneers such as Locard indicated that fingerprint features should be assessed as a function of their relative rarity (allowing also for a proper consideration of their clarity). ${ }^{608}$ Currently, however, the weighting of individual features is mainly based upon subjective, experience-based judgments of the probability associated with their occurrence. In fact, in one study, latent print examiners did not provide consistent rankings of how frequently

${ }^{608}$ Locard, E. "La Preuve Judiciaire par les Empreintes Digitales.” Archives d'Anthropologie Criminelle, de Médecine Légale et de Psychologie Normale et Pathologique, 29 (1914): 321 - 48. 
fingerprint features occur. ${ }^{609}$ No clear consensus exists among practitioners as to the reproducibility and perceived contribution of Level 3 Detail features appearing in both latent and the exemplar print. ${ }^{610}$ Carefully collected data should be used to provide more accurate assessments that can inform the weighting of features in the interpretive process.

This statistical data also could be incorporated into formal probabilistic models of various kinds (see Chapter 4). In addition to providing both a firmer foundation for and opportunities to improve the judgments of examiners, such modeling can supply objective probabilities that would be useful to judges or juries (Chapter 6). Before that can happen, however, large datasets will be needed to validate the emerging models more fully. The Working Group therefore recommends:

To facilitate the validation of probabilistic models and other statistical research, the federal government should create large, anonymous databases of exemplars and latent prints.

(Recommendation 4.2)

Visual acuity is vital to the examination process, and section 10.3 included a recommendation for medical monitoring. To permit the most effective monitoring, the Working Group also recommends:

The federal government should support research to determine the most appropriate tests of visual function for friction ridge examiners. (Recommendation 7.2 in part)

As discussed in Chapter 4 and section 10.4, AFIS technology has proven to be of great value in efficiently locating candidate exemplars for examiners to compare to a questioned print. The design of these systems could be improved in several respects.

The federal government should support research programs to improve automated fingerprint identification systems. Such programs could address the following issues:

a. Expanding the algorithms used to match prints to account for the fact that the diagnostic value of minutiae depends on the region in which they are located;

b. Making fingerprint and palm print databases interoperable among local, state, and federal automated identification systems; and

c. Increasing compatibility between automated identification systems and other latent print software tools, including digital enhancement programs, probability calculation programs, and automated quality assessment programs. (Recommendation 4.1)

The reference above to automated quality assessment programs relates to Recommendation 3.9 for research into the interpretative process. In the experience of the Working Group, the initial quality assessment of the latent print is often the weak link in the analytical process, as examiners are susceptible to making one of two types of errors. In the first type of error, the examiner prematurely discards a print that should be deemed suitable for further analysis and comparison, possibly resulting in a missed identification. In the second type of error, the

\footnotetext{
${ }^{609}$ Osterburg, J. "An Inquiry into the Nature of Proof. The Identity of Fingerprints." Journal of Forensic Sciences, 9 , no. 4 (1964): $413-27$.

${ }^{610}$ Anthonioz, A., N. Egli, C. Champod, et al. "Level 3 Details and Their Role in Fingerprint Identification: A Survey Among Practitioners." Journal of Forensic Identification, 58, no. 5 (2008): 562 - 89.
} 
examiner continues to evaluate a latent print that ought to be deemed unsuitable for further processing. This outcome results in wasted time and might even contribute to an erroneous identification. Although efforts to develop automated quality assessment programs have had limited success, a concerted effort to automate the quality assessment step, at least partially, is worthwhile. In addition to Recommendation 3.9(a), the Working Group proposes:

The federal government should continue funding research into automation of the initial quality assessment step in latent print analysis. (Recommendation 4.4)

Latent print analysis provides perhaps the single most common type of forensic science evidence. It is critical to the successful operation of the criminal justice system. The recommendations listed above do not exhaust this report's suggestions for improving the understanding and management of human factors issues in this vital work. Related suggestions, explanations, and ideas are found in each chapter. Translating these ideas into practice will make the analysis and presentation of a crucial source of information even more effective and reliable. 


\section{Appendix 10-A: List of Recommendations}

Recommendation 3.1: A report and contemporaneous supporting notes or materials should document the examination to make the interpretive process as transparent as possible. Although the degree of detail may vary depending on the perceived complexity of the comparison, documentation should, at a minimum, be sufficient to permit another examiner to assess the accuracy and validity of the initial examiner's assessment of the evidence.

Recommendation 3.2: Modifications to the results of any stage of latent print analysis (e.g., feature selection, utility assessment, discrepancy interpretation) after seeing a known exemplar should be viewed with caution. Such modifications should be specifically documented as having occurred after comparison had begun.

Recommendation 3.3: Procedures should be implemented to protect examiners from exposure to extraneous (domain-irrelevant) information in a case.

Recommendation 3.4: Each agency or forensic service provider should define "suitable" or "sufficient" in its standard operations procedures. These guidelines should be as explicit as possible about what is expected for sufficiency determinations at different stages of the latent print examination process.

Recommendation 3.5: Because statistical information plays a fundamental role in weighting latent print feature evidence, training should include the best available empirical information and should educate examiners about probabilistic reasoning in using that information.

Recommendation 3.6: When comparing latent prints to exemplars generated through AFIS searches, examiners must recognize the possibility and dangers of incidental similarity.

Adjustments such as a higher decision threshold, stricter tolerances for differences in appearance, and explicit feature weighting need to be considered. Modifying quality assurance practices for this scenario also should be considered.

Recommendation 3.7: Because empirical evidence and statistical reasoning do not support a source attribution to the exclusion of all other individuals in the world, latent print examiners should not report or testify, directly or by implication, to a source attribution to the exclusion of all others in the world.

Recommendation 3.8: The friction ridge examiner community and other experts should determine under what circumstances a qualified rather than an absolute conclusion is warranted.

Recommendation 3.9: The federal government should support a research program that aims to:

a. Develop measures and metrics relevant to the analysis of latent prints;

b. Use such metrics to assess the reproducibility, reliability, and validity of various interpretive stages of latent print analysis; and

c. Identify key factors related to variations in performance of latent print examiners during the interpretation process. 
Recommendation 4.1: The federal government should support research programs to improve automated fingerprint identification systems. Such programs could address the following issues:

a. Expanding the algorithms used to match prints to account for the fact that the diagnostic value of minutiae depends on the region in which they are located;

b. Making fingerprint and palm print databases interoperable among local, state, and federal automated identification systems; and

c. Increasing compatibility between automated identification systems and other latent print software tools, including digital enhancement programs, probability calculation programs, and automated quality assessment programs.

Recommendation 4.2: To facilitate the validation of probabilistic models and other statistical research, the federal government should create large, anonymous databases of exemplars and latent prints.

Recommendation 4.3: The latent print examiner community should expand the training of examiners in elementary probability theory to enable examiners to properly utilize the output of probabilistic models.

Recommendation 4.4: The federal government should continue funding research into automation of the initial quality assessment step in latent print analysis.

Recommendation 5.1: The report of the examination should ensure that the findings and their limitations are intelligible to non-experts.

Recommendation 5.2: A report should:

a. Identify the latent print examiner(s);

b. Describe the items submitted to the examiner(s);

c. List the procedures used by the examiner to develop, visualize, or enhance the friction ridge impressions;

d. List all comparisons conducted;

e. State all conclusions with the method used to reach them;

f. Note any important limitations to the conclusions;

g. Indicate whether a verification was made and whether there was any conflict of opinion among examiners prior to the reported conclusions;

h. Note (or refer to external documentation of) any information about the case that the examiner(s) received;

i. Note the existence of additional documentation; and

j. Define important technical terms, either explicitly or by reference to an authoritative, readily available source.

Recommendation 6.1: The trial preparation process should address the presentation of technical information in lay terms, the organization of the direct examination, possible cross-examination, and the possible use of visual aids.

Recommendation 6.2: Forensic service providers should adopt codes of ethics that require testifying in a nonpartisan manner; answering questions from both the prosecution and the 
defense directly, accurately, and fully; and providing appropriate scientific information before, during, and after trial.

Recommendation 6.3: A testifying expert should be familiar with the literature related to error rates. A testifying expert should be prepared to describe the steps taken in the examination process to reduce the risk of observational and judgmental error. The expert should not state that errors are inherently impossible or that a method inherently has a zero error rate.

Recommendation 6.4: An agency that employs latent print examiners should establish requirements and guidelines for reporting, documentation, and testimony. The agency should review a sample of every examiner's case reports, documentation, and testimony and affidavits at least annually to verify compliance with these requirements and guidelines.

Recommendation 7.1: A structured approach that involves users throughout the entire design and implementation process should be followed when designing technology systems and the physical work environment. In addition, forensic service providers should perform usability testing to optimize user performance and user satisfaction before deployment.

Recommendation 7.2: Forensic service providers should institute medical surveillance for examiners with respect to activities specific to friction ridge examination. Corrective measures should be taken when appropriate and recorded. The program should include a baseline visual examination followed by annual vision testing to detect vision deficiencies that may affect interpretation of the evidence. The federal government should support research to determine the most appropriate tests of visual function for friction ridge examiners.

Recommendation 8.1: The federal government should consider funding research to determine what educational and cognitive abilities should be prerequisites for training a latent print examiner. Forensic service providers should have systematic training programs that include these abilities as well as education in the scientific method, reading and understanding relevant scientific literature, communication skills, and methods for logically developing conclusions.

Recommendation 8.2: The federal government should develop a forensic latent print examiner scholarship program to:

a. Fund the post-hire training program and, upon successful completion, provide tuition reimbursement, loan forgiveness, or other financial assistance to degreed latent print examiner trainees working in state, local, or private (not-for-profit) forensic laboratories; and

b. Reimburse the employer for a percentage of the salaries during the training period.

Recommendation 8.3: Agencies should establish formal mentoring programs that define the responsibilities of the mentor, trainee, and supervisor.

Recommendation 8.4: The federal government should establish an expert group to develop a latent print educational textbook, practical exercises, and assessment tests (print and online) in consultation with professional organizations, senior latent print examiners, researchers with content knowledge, experienced trainers, and professionals experienced in curriculum development. 
Recommendation 8.5: Training materials should include topics beyond the technical aspects of friction ridge analysis, such as:

a. Documentation of work and case notes and written and oral communication;

b. Professional ethics;

c. Human factors issues such as fatigue, bias, cognitive influences, perceptual influences, and error;

d. Research methods; and

e. Legal and ethical aspects of expert testimony.

Recommendation 8.6: The federal government should support a clearinghouse of materials from existing training programs as well as publications on latent print identification.

Recommendation 8.7: Forensic service providers should require personnel to participate in continuing education and to become certified through an accredited program. Certifying bodies should review current certification tests with the aid of professionals in test design and psychometrics.

Recommendation 8.8: Certifying bodies should ensure that examiners have been trained to competency. Laboratory accrediting bodies should evaluate the effectiveness of an agency's training program.

Recommendation 9.1: Management should foster a culture in which it is understood that some human error is inevitable and that openness about errors leads to improvements in practice.

Recommendation 9.2: Management should employ a system to identify and track errors and their causes.

Recommendation 9.3: All forensic service providers should be accredited by a recognized accrediting body.

Recommendation 9.4: Management should establish policies and procedures for case review and conflict resolution, corrective action, and preventive measures.

Recommendation 9.5: The latent print community should develop and implement a comprehensive testing program that includes competency testing, certification testing, and proficiency testing. 


\section{Bibliography}

The following list includes all references cited in Latent Print Examination and Human Factors: Improving the Practice through a Systems Approach. The numbers in brackets after each citation indicate the page in the report where the reference can be found. A list of court cases cited in this report follows the full reference list.

Abramov, I. and J. Gordon. "Color Vision Panel Tests: A Metric for Interpreting Numeric Analytic Indices." Optometry \& Vision Science, 86 (2009): 146. [148]

Aickin, M. and D. Kaye. "Some Mathematical and Legal Considerations in Using Serologic Tests to Prove Paternity." Inclusion Probabilities in Parentage Testing, 53 (1983): 167. [134]

Aitken, C. and F. Taroni. Statistics and the Evaluation of Evidence for Forensic Scientists. 2nd ed. John Wiley \& Sons, 2004. [18, 69, 70, 97, 133, 134]

Allen, M. and W. Yen. Introduction to Measurement Theory. Waveland Press, 2002. [25]

Allison, R. and K. Uhl. "Influence of Beer Brand Identification on Taste Perception." Journal of Marketing Research, 1 (1964): 36. [11]

Amax Coal Co. v. Beasley, 957 F.2d 324, 328 (7th Cir.1992). [119]

American Academy of Forensic Sciences, Forensic Science Education Programs Accreditation Commission. Accreditation Standards. American Academy of Forensic Sciences, revised July 22, 2011. [165, 169]

American Bar Association. Standards for Criminal Justice: DNA Evidence. 3rd ed. American Bar Association, 2007. [90, 91, 94]

American College of Emergency Physicians. Benchmarking in Emergency Medicine, March 1997. Accessed November 29, 2011. http://www.acep.org/content.aspx?id=34362. [177]

American Optometric Association. Comprehensive Adult Eye and Vision Examination. American Optometric Association, 2005. [148]

American Society of Crime Laboratory Directors. Guidelines for Forensic Laboratory Management Practices. American Society of Crime Laboratory Directors, 1987. [96, 98]

American Society of Crime Laboratory Directors/Laboratory Accreditation Board. ASCLD/LAB Guiding Principles of Professional Responsibility for Crime Laboratories and Forensic Scientists. Version 1.1. 2009. [42 (Principle 15), 44 (Principle 2), 115 (Principle 19), 117 (Principle 18)]

American Society of Crime Laboratory Directors/Laboratory Accreditation Board. ASCLD/ LAB-International Supplemental Requirements for the Accreditation of Forensic Science Testing Laboratories 2011 Edition. American Society of Crime Laboratory Directors/ Laboratory Accreditation Board, approved September 11, 2010. [184, 186]

Annotation, Admissibility and Weight of Fingerprint Evidence Obtained or Visualized by Chemical, Laser, and Digitally Enhanced Imaging Processes, ALR 5th Alert, 110 (2003): 213. [82]

Anthonioz, A., N. Egli, C. Champod, et al. "Level 3 Details and Their Role in Fingerprint Identification: A Survey Among Practitioners." Journal of Forensic Identification, 58, no. 5 (2008): 562-89. [55, 205]

Ashbaugh, D. "Poroscopy." RCMP Gazette, 45, no. 2 (1983): 12. [61] 
Ashbaugh, D. Quantitative-Qualitative Friction Ridge Analysis: An Introduction to Basic and Advanced Ridgeology. CRC Press, 1999. [4, 46, 64, 68, 167]

Ashbaugh, D. Ridgeology: Modern Evaluative Friction Ridge Identification. Royal Canadian Mounted Police, 1999. [164]

Association of Firearm and Tool Mark Examiners. Theory of Identification as it Relates to Toolmarks. 5th ed., ver. 5.070207. 2007. [17]

Association of Forensic Science Providers. "Standards for the Formulation of Evaluative Forensic Science Expert Opinion.” Science and Justice, 49, no. 3 (2009): 161-64. [70, 134]

Augustine, J. "ED Wait Times Improving for the First Time in 40 Years." Improving Health Care, Press Ganey. February 21, 2011. [177]

Bagot, G. "Ergonomics in the Laboratory Environment." Nursing Management, 25 1994): 50. [140]

Baker, D., R. Day, and E. Salas. "Teamwork as an Essential Component of High-Reliability Organizations." Health Services Research, 41 (August 2006): 1576-98. [181]

Barnes, J. "History." In The Fingerprint Sourcebook. National Institute of Justice, 2011. [vi]

Barnhardt, C., S. Clock, B. Demmer, et al. "Color Vision Screening for Individuals with Intellectual Disabilities: A Comparison Between the Neitz Test of Color Vision and Color Vision Testing Made Easy." Optometry, 77 (2006): 211. [148]

Bartlett, H., L. Davies, and F. Eperjesi. "The Macular Mapping Test: A Reliability Study.” BMC Ophthalmology, 10 (2005): 18. [148]

Bazen, A. and R. Veldhuyis. "Likelihood-Ratio-Based Biometric Verification." In IEEE Transactions on Circuits and Systems Video Technology, 14 (2004): 85. [84]

Bazerman, M. and M. Neale. Negotiating Rationally. Free Press, 1994. [23]

Berger, J. Statistical Decision Theory and Bayesian Analysis. 2nd ed. Springer, 1984. [25]

Berger, M. "Laboratory Error Seen Through the Lens of Science and Policy." University of California Davis Law Review, 30 (1997): 1081. [125]

Bertino, A. and P. Bertino. Forensic Science: Fundamentals and Investigations. South-Western Educational Publishing, 2009. [vi]

Bevington, P. and D. Robinson. Data Reduction and Error Analysis for the Physical Sciences. 3rd ed. McGraw-Hill, 2002. [21]

Bickel, P. and K. Doksum. Mathematical Statistics. Holden-Day, 1977. [34]

Biedermann, A., S. Bozza, and F. Taroni. "Decision Theoretic Properties of Forensic Identification: Underlying Logic and Argumentative Implications.” Forensic Science International, 177 (2008): 120. [15, 68]

Bolt, R., F. Cooper, E. David, et al. "Speaker Identification by Speech Spectrographs: Some Further Observations." Journal of the Acoustical Society of America, 54 (1973): 531. [125]

Boyce, P., C. Hunter, and O. Howlette. The Benefits of Daylight Through Windows. Lighting Research Center, Rensselaer Polytechnic Institute, 2003. [153]

Brady v. Maryland, 373 U.S. 83, 87 (1963). [91]

Briscoe v. Virginia, 130 S.Ct. 1316 (2010). [94]

Buckleton, J., C. Triggs, and C. Champod. "An Extended Likelihood Ratio Framework for Interpreting Evidence.” Science \& Justice, 46 (2006): 69. [133]

Budowle, B., M. Bottrell, S. Bunch, et al. "A Perspective on Errors, Bias, and Interpretation in the Forensic Sciences and Direction for Continuing Advancement." Journal of Forensic Sciences, 54 (July 2009): 803. [10, 11, 117, 126, 200] 
Budowle, B., J. Buscaglia, and R. Perlman. "Review of the Scientific Basis for Friction Ridge Comparisons as a Means of Identification: Committee Findings and Recommendations." Forensic Science Communications, 8 (January 2006). [96]

Budowle, B., R. Chakraborty, G. Carmody, et al. "Source Attribution of a Forensic DNA Profile." Forensic Science Communications, 2, no. 3 (2000). [131]

Bullcoming v. New Mexico, 131 S.Ct. 2705 (2010). [93]

Bunche v. State, 5 So.3d 38, 40 n.1 (Fla. Dist. Ct. App. 2009). [127]

Busey, T. and I. Dror. "Special Abilities and Vulnerabilities in Forensic Expertise." In The Fingerprint Sourcebook. National Institute of Justice, 2011. [11]

Butler, J. Fundamentals of Forensic DNA Typing. Academic Press, 2010. [33]

Butt, L. Letter from the Chairman, Scientific Working Group on Friction Ridge Analysis, Study and Technology. In Regards to: Position Clarification. June 29, 2010. Accessed June 3, 2011. http://www.swgfast.org/Comments-Positions/SWGFAST_NAS_Position_ Clarification.doc. [17]

Camp, R. Business Process Benchmarking: Finding and Implementing Best Practices. ASQC Quality Press, 1995. [177]

Carayon, P., C. Alvarado, and A. Hundt. Reducing Workload and Increasing Patient Safety through Work and Workspace Design. Center for Quality and Productivity Improvement, University of Wisconsin-Madison, 2003. [154]

Carmichael v. Samyang Tire, Inc., 131 F.3d 1433, 1435-36 (1997). [121]

Carter v. State, 766 N.E.2d 377 (Ind. 2002). [123]

Champod, C. "Fingerprint Examination: Towards More Transparency." Law, Probability \& Risk, 7 (2008): 111. [32, 34, 114, 124, 133]

Champod, C. "Friction Ridge Examination (Fingerprints): Interpretation of." In Wiley Encyclopedia of Forensic Sciences edited by A. Moenssens and A. Jamieson, vol. 3, pp. 1508-11. J. Wiley \& Sons, 2009. [134]

Champod, C. "Identification and Individualization." In Wiley Encyclopedia of Forensic Sciences, edited by A. Moenssens and A. Jamieson, vol. 3, pp. 1508-11. J. Wiley \& Sons, 2009. [68]

Champod, C. Reconnaissance Automatique et Analyse Statistique des Minuties sur les Empreintes Digitales. PhD Thesis. Institut de Police Scientifique et de Criminologie, Université de Lausanne, Lausanne, Suisse, 1996. [2, 60]

Champod, C. and I. Evett. "Evidence Interpretation: A Logical Approach." In Wiley Encyclopedia of Forensic Sciences, edited by A. Moenssens and A. Jamieson, vol. 2, pp. 968-76. J. Wiley \& Sons, 2009. [70]

Champod, C., C. Lennard, P. Margot, et al. Fingerprints and Other Ridge Skin Impressions. CRC Press, 2004. [57]

Champod, C. and P. Margot. "Computer Assisted Analysis of Minutiae Occurrences on Fingerprints." In Proceedings of the International Symposium on Fingerprint Detection and Identification, 1995, 305-18. [61, 84]

Chapanis, A., W. Garner, and C. Morgan. Applied Experimental Psychology: Human Factors Engineering Design. Wiley, 1985. [142]

Chen, Y. and A. Jain. "Beyond Minutiae: A Fingerprint Individuality Model with Pattern, Ridge, and Pore Features." In Proceedings, International Conference on Biometrics (ICB), Advances in Biometrics, Third International Conference, Alghero, Italy, June 2-5, 2009. [83] 
Chen, Y. and A. Jain. "Dots and Incipients: Extended Features for Partial Fingerprint Matching." Paper presented at the Biometrics Symposium 2007. [62]

Chignell, M. and J. Waterworth. Multimedia, Handbook of Human Factors and Ergonomics. 2nd ed. John Wiley \& Sons, 1997. [140]

Cocci, S., K. Namaisvayam, and P. Bordi. "An Investigation of Ergonomic Design and Productivity Improvements in Foodservice Production Tables." Foodservice Research International, 16 (2005): 53. [149]

Cohen, S. and M. Rodriguez. "Pathways Linking Affective Disturbances and Physical Disorders." Health Psychology, 14 (1995): 374. [156]

Cole, S. "Comment on Scientific Validation of Fingerprint Evidence Under Daubert." Law, Probability \& Risk, 7, no. 119 (2008): 123. [122, 124]

Cole, S. "Fingerprinting: The First Junk Science?" Oklahoma City University Law Review, 28, no. 73 (2003): 88. [118]

Cole, S. "More Than Zero: Accounting for Error in Latent Fingerprint Identification." Journal of Criminal Law and Criminology, 95 (2005): 1033. [32, 33]

Cole, S. Suspect Identities: A History of Fingerprinting and Criminal Identification. Harvard University Press, 2001. [vi]

Cole, S. "Where the Rubber Meets the Road: Thinking About Expert Evidence as Expert Testimony." Villanova Law Review, 52 (2007): 803. [129]

Cole, S., M. Welling, R. Dioso-Villa, et al. "Beyond the Individuality of Fingerprints: A Measure of Simulated Computer Latent Print Source Attribution Accuracy." Law, Probability \& Risk, 7 (2008): 165. [15]

Collaborative Testing Services, Inc. CTS Statement on the Use of Proficiency Testing Data for Error Rate Determination. Collaborative Testing Services, Inc, March 30, 2010. [33]

Collins, B. Windows and People: A Literature Survey, Psychological Reaction to Environments With and Without Windows. U.S. Dept. of Commerce, National Bureau of Standards, 1975. [153]

Commonwealth v. Carter, 932 A.2d 1261 (Pa. 2007). [92]

Commonwealth v. Drayton, 434 N.E.2d 997 (Mass. 1982). [132]

Commonwealth v. Gambora, 933 N.E.2d 50, 56 (Mass. 2010). [130]

Commonwealth v. Pytou Heang, 942 N.E.2d 927 (Mass. 2011). [120, 122]

Cooey v. Strickland, 589 F.3d 210, 231 (6th Cir. 2009). [119]

Cook, R., I. Evett, G. Jackson, et al. "A Model for Case Assessment and Interpretation.” Science \& Justice, 38 (1998): 151. [134]

Cooney, L. "Latent Print Training to Competency: Is it Time for a Universal Training Program?" Journal of Forensic Identification, 60 (2010): 223-58. [165, 169]

Council Framework Decision 2009/905/JHA of 30 November 2009 on Accreditation of Forensic Service Providers Carrying out Laboratory Activities. "Acts Adopted Under the Title VI of the EU Treaty." Official Journal of the European Union, 52, (December 9, 2009): 14-16. [183]

Cowger, J. Friction Ridge Skin: Comparison and Identification of Fingerprints. CRC Press, 1992. [65, 167]

Cowger, J. "Moving Towards Professionalization of Latent Print Examiners." Journal of Forensic Sciences, 24 (1979): 591-95. [165]

Dass, S., Y. Zhu, and A. Jain. "Statistical Models for Assessing the Individuality of Fingerprints." Proceedings. Fourth IEEE Workshop on Automatic Identification Advanced Technology, 2005. [83] 
Daubert v. Merrell Dow Pharmaceuticals, Inc., 509 U.S. 579 (1993). [22, 36 (at 594), 86, 118, 121]

Deming, W. Out of the Crisis. Massachusetts Institute of Technology, Center for Advanced Educational Services, 1986. [172]

Dismukes, K., G. Young, and R. Sumwalt. Cockpit Interruptions and Distractions: Effective Management Requires A Careful Balancing Act. National Aeronautics and Space Administration, 1998. [155]

Dorai, C., N. Ratha, and R. Bolle. "Dynamic Behavior Analysis in Compressed Fingerprint Videos." IEEE Transactions on Circuits and Systems for Video Technology, 14, no. 1 (2004): 58-73. [65]

Doubilet, P. and P. Herman. "Interpretation of Radiographs: Effect of Clinical History." American Journal of Roentgenology, 137, no. 5 (1981): 1055. [11]

Dror, I. "The Paradox of Human Expertise: Why Experts Can Get It Wrong." In The Paradoxical Brain, edited by N. Kapur. Cambridge University Press, 2011. [11]

Dror, I., C. Champod, G. Langenburg, et al. "Cognitive Issues in Fingerprint Analysis: Inter- and Intra-Expert Consistency and the Effect of a 'Target' Comparison." Forensic Science International, 208 (2011): 10-17. [49, 51, 52]

Dror, I. and D. Charlton. "Why Experts Make Errors.” Journal of Forensic Identification, 56, no. 4 (2006): 600-16. [12, 50, 71, 75]

Dror, I., D. Charlton, and A. Peron. "Contextual Information Renders Experts Vulnerable to Making Erroneous Identifications.” Forensic Science International, 156, no. 1 (2006): 74-78. [12, 43, 50, 52, 71]

Dror, I. and S. Cole. “The Vision in 'Blind' Justice: Expert Perception, Judgment, and Visual Cognition in Forensic Pattern Recognition.” Psychonomic Bulletin \& Review, 17 (2010): 161. [11, 12]

Dror, I. and J. Mnookin. "The Use of Technology in Human Expert Domains: Challenges and Risks Arising From the Use of Automated Fingerprint Identification Systems in Forensic Science." Law, Probability \& Risk, 9 (2010): 47-67. [62, 87, 97]

Dror, I., A. Peron, S. Hind, et al. "When Emotions Get the Better of Us: The Effect of Contextual Top-Down Processing on Matching Fingerprints." Applied Cognitive Psychology, 19 (2005): 799-809. [50]

Dror, I. and R. Rosenthal. "Meta-Analytically Quantifying the Reliability and Biasability of Forensic Experts.” Journal of Forensic Sciences, 53 (July 2008): 900. [11, 12]

Dror, I., K. Wertheim, P. Fraser-Mackenzie, et al. "The Impact of Human-Technology Cooperation and Distributed Cognition in Forensic Science: Biasing Effects of AFIS Contextual Information on Human Experts." Journal of Forensic Sciences (forthcoming). [78]

Dyck, L. "High Reliability Organization (HRO) in Practice.” Technical Support. January 2007. [181]

Edwards, H. "Solving the Problems that Plague the Forensic Science Community." Jurimetrics Journal of Law, Science, and Technology, 50 (2009): 9. [92]

Egli, N. "Interpretation of Partial Fingermarks Using an Automated Fingerprint Identification System." PhD thesis, École des Sciences Criminelles, University of Lausanne, 2009. [61]

Egli, N., C. Champod, and P. Margot. "Evidence Evaluation in Fingerprint Comparison and Automated Fingerprint Identification Systems - Modelling Within Finger Variability." Forensic Science International, 167 (2007): 189. [46, 84, 87, 135]

215 Latent Print Examination and Human Factors: Improving the Practice through a Systems Approach The Report of the Expert Working Group on Human Factors in Latent Print Analysis 
Emergency Department Benchmarking Alliance. About Us. Accessed November 29, 2011. http://edbenchmarking.org/index.php. [177]

European Network of Forensic Science Institutes Standing Committee for Quality and Competence. Performance Based Standards for Forensic Science Practitioners. European Network of Forensic Science Institutes Standing Committee for Quality and Competence, July 2004. [94, 96, 98, 100, 117]

Evans, J. Bias in Human Reasoning: Causes and Consequences. Psychology Press, 1989, p. 41. [10]

Evett, I. and J. Buckleton. "Statistical Analysis of STR Data." Advances in Forensic Haemogenetics, 6 (1996): 79. [135]

Evett, I., G. Jackson, J. Lambert, et al. "The Impact of the Principles of Evidence Interpretation on the Structure and Content of Statements." Science \& Justice, 40 (2000): 233. [134]

Evett, I. and R. Williams. "A Review of the Sixteen Points Fingerprint Standard in England and Wales.” Fingerprint Whorld, 82 (1995): 125-43. [49, 50, 51, 52, 75]

Evett, I. and R. Williams. "A Review of the Sixteen Points Fingerprint Standard in England and Wales." Journal of Forensic Identification, 46, no. 1 (1996): 49. [125]

Ex parte Dolvin, 391 So.2d 677, 679 (Ala. 1980). [123]

Ezzelle, J., I. Rodriguez-Chavez, J. Darden, et al. "Guidelines on Good Clinical Laboratory Practice: Bridging Operations Between Research and Clinical Research Laboratories." Journal of Pharmaceutical and Biomedical Analysis, 46 (January 2008): 18. [140]

Faigman, D., D. Kaye, M. Saks, et al. Modern Scientific Evidence: The Law and Science of Expert Testimony. 1st ed. West Publishing Company, 1997. [113, 115, 129]

Fang, L., D. Wyon, G. Clausen, et al. "Impact of Indoor Air Temperature and Humidity in an Office on Perceived Air Quality, SBS Symptoms and Performance.” Indoor Air, 14, supp. 7 (2004): 74-81. [152]

Federal Bureau of Investigation. "Integrated Automated Fingerprint Identification System." Accessed April 23, 2011. http://www.fbi.gov/aboutus/cjis/fingerprints_biometrics/iafis/iafis. [78]

Federal Bureau of Investigation. The Science of Fingerprints: Classification and Uses. Department of Justice, 1979. [163]

Federal Rules of Evidence 403. [119, 123]

Federal Rules of Evidence 702. [115]

Federspeil, C., G. Lui, M. Lahiff, et al. "Worker Performance and Ventilation: Analyses of Individual Data for Call-Center Workers." Indoor Air, 14 (2002): 41. [152]

Feinberg, S., ed. The Evolving Role of Statistical Assessments as Evidence in the Courts. Springer, 1989. [115, 116]

Felder, R. "Reaching the Second Tier: Learning and Teaching Styles in College Science Education." Journal of College Science Teaching, 23 (1993): 286. [151]

Feng, J., A. Jain, and A. Ross. "Detecting Altered Fingerprints." In 2010 20th International Conference on Pattern Recognition (ICPR), August 2010, 1622-25. [47]

Flaherty, M. "400 Drunken-Driving Convictions In D.C. Based On Flawed Test, Official Says.” The Washington Post, June 10, 2010. [13]

Frye v. United States, 293 F. 1013 (D.C. Cir. 1923). [86, 120]

Gaensslen, R. "How Do I Become a Forensic Scientist?, Educational Pathways to Forensic Science Careers." Analytical and Bioanalytical Chemistry, 376 (2003): 1151-55. [164]

Galton, F. Finger Prints. 1892. Reprint, William S. Hein \& Co., 2003. [40, 83] 
Garrett, B. Convicting the Innocent: Where Criminal Prosecutions Go Wrong. Harvard University Press, 2011. [92]

Giannelli, P. "Independent Crime Laboratories: The Problem of Motivational and Cognitive Bias." Utah Law Review, 247 (2010): 251. [12]

Giannelli, P. and E. Imwinkelried. Scientific Evidence. 4th ed. Matthew Bender, 2007. [113, 115, 129]

Gill, P., J. Curran, C. Neumann, et al. "Interpretation of Complex DNA Profiles Using Empirical Models and a Method to Measure Their Robustness." Forensic Science International, 2 (2008): 9. [135]

Gonzalez-Rodriguez, J., J. Fierrez-Aguilar, D. Ramos-Castro, et al. "Bayesian Analysis of Fingerprint, Face and Signature Evidences with Automatic Biometric Systems." Forensic Science International, 155 (2005): 126. [84]

Goodman-Delahunty, J., P. Granhag, M. Hartwig, et al. 'Insightful or Wishful: Lawyers' Ability to Predict Case Outcomes." Psychology, Public Policy, and Law, 16 (2010): 133. [23]

Grieve, D. "The Identification Process: Traditions in Training." Journal of Forensic Identification, 40 (1990): 199. [163, 164]

Grogan, E., R. Stiles, D. France, et al. "The Impact of Aviation-Based Teamwork Training on the Attitudes of Health-Care Professionals." Journal of the American College of Surgeons, 199 (December 2004): 843. [140]

Gross, S. "Expert Evidence.” Wisconsin Law Review, 1113 (1991): 1151. [116]

Gupta, S. "Statistical Survey of Ridge Characteristics.” International Criminal Police Review, 218 (1968): 130. [59]

Gutièrrez, E., V. Galera, J. Martínez, et al. "Biological Variability of the Minutiae in the Fingerprints of a Sample of the Spanish Population." Forensic Science International, 172 (2007): 98. [58]

Gutowski, S. "Error Rates in Fingerprint Examination: The View in 2006." The Forensic Bulletin, Autumn 2006, 18. [125]

Haber, L. and R. Haber. Challenges to Fingerprints: A Guidebook for Prosecution and Defense and Examiners. Lawyers and Judges Publishing Co., 2009. [51, 96]

Haber, L. and R. Haber. "Scientific Validation of Fingerprint Evidence Under Daubert." Law, Probability \& Risk, 7 (2008): 87. [33, 120, 124 (page 98)]

Hanley, J. and A. Lippman-Hand. "If Nothing Goes Wrong, Is Everything All Right? Interpreting Zero Numerators." Journal of the American Medical Association, 249 (1983): 1743. [34]

Heinrich, H., D. Peterson, and N. Roos. Industrial Accident Prevention: A Safety Management Approach. 1st ed. 1931. [174]

Hendrick, H. "Determining the Cost-Benefits of Ergonomics Projects and Factors That Lead to Their Success." Applied Ergonomics, 34 (2003): 419. [156]

Henning, R., P. Jacques, G. Kissel, et al. "Frequent Short Rest Breaks From Computer Work: Effects on Productivity and Well-Being at Two Field Sites." Ergonomics, 40 (January 1997): 78. [140]

Henriksen, K., E. Dayton, M. Keyes, et al. "Understanding Adverse Events: A Human Factors Framework." In Patient Safety and Quality: An Evidence-Based Handbookfor Nurses edited by Ronda G. Hughes. Agency for Healthcare Research and Quality, 2008. [141]

Henry, E. Classification and Uses of Finger Prints. 4th ed. George Routledge and Sons, 1900. [56] 
Henson, S. "Brady Violations by DPS Fingerprint Examiners? Is Fingerprint Examination even Science?" Grits for Breakfast. Blog post, October 9, 2010. Accessed November 25, 2011. http://gritsforbreakfast.blogspot.com/2010/10/brady-violations-by-dps-fingerprint.html. [92]

Heschong Mahone Group. Windows and Offices: A Study of Office Worker Performance and the Indoor Environment - CEC PIER 2003. 2003. [153]

Hicklin, A., et al. "Latent Fingerprint Quality: A Survey of Examiners." Journal of Forensic Identification, Jul-Aug 2011, 385-417. [87]

Hickner, J., D. Graham, N. Elder, et al. "Testing Process Errors and Their Harms and Consequences Reported From Family Medicine Practices: A Study of the American Academy of Family Physicians National Research Network." Quality and Safety in Health Care, 17 (2008): 194. [24]

Hicks v. Schurr, 871 F.2d 255 (8th Cir. 1982). [132]

Hofer, T., E. Kerr, and R. Hayward. "What Is an Error?" Effective Clinical Practice, 3 (2000): 261. [21, 25]

Hoffrage, U. "Overconfidence." In Cognitive Illusions: A Handbook on Fallacies and Biases in Thinking, Judgment, and Memory. Psychology Press, 2004. [23]

Hopp, P., C. Smith, B. Clegg, et al. "Interruption Management: The Use of Attention-Directing Tactile Cues." Human Factors, 47 (2005): 1. [140]

Houck, M. and J. Siegel. Fundamentals of Forensic Science. 2nd ed. Academic Press, 2010. $[132,133]$

Hummel, K. and J. Gerchow, ed. Biomathematical Evidence of Paternity. 1982. Joint AMAABA Guidelines: Present Status of Serologic Testing in Problems of Disputed Parentage. Family Law Quarterly. 10 (1976): 247. [134]

Hutchins, E. "How a Cockpit Remembers Its Speeds." Cognitive Science, 19 (1995): 265. [23]

Imwinkelried, E. and D. Kaye. "DNA Typing: Emerging or Neglected Issues." Washington Law Review, 76 (2001): 413. [126, 127]

International Association for Identification. Certification Program Operations Manual. Version 2010_H_I. International Association for Identification, July 10, 2010. [181]

International Association for Identification. Constitution, Article 1, Section 1.03f. International Association for Identification, 2011. [163]

International Association for Identification. Friction Ridge Skin Identification Training Manual. International Association for Identification, 2006. [164, 167]

International Association for Identification. IAI Resolution 2010-18. International Association for Identification, July 16, 2010. [73, 82, 118]

International Association for Identification. "Resolution VII Amended." Identification News, 3 (August 1980). [118]

International Association for Identification. "Resolution VII.” Identification News, 29 (August 1979). [82, 118]

International Association for Identification: Scientific Working Group on Imaging Technology (SWGIT). Accessed April 23, 2011. http://www.theiai.org/guidelines/swgit/index.php. [81]

International Organization for Standardization. Acoustics - Noise Emitted by Machinery and Equipment-Determination of Emission Sound Pressure Levels at a Work Station and at Other Specified Positions in an Essentially Free Field over a Reflecting Plane with Negligible Environmental Corrections, ISO 11201:2010. International Organization for Standardization, 2010. [154] 
International Organization for Standardization. Building Environment Design-Indoor Air Quality-Methods of Expressing the Quality of Indoor Air for Human Occupancy, ISO 16814:2008. International Organization for Standardization, 2008. [152]

International Organization for Standardization. Ergonomic Principles Related to Mental

Workload - Part 2: Design Principles, ISO 10075-2:1996. International Organization for

Standardization, 1996. [151]

International Organization for Standardization. Ergonomic Requirements for Office Work with

Visual Display Terminals (VDTs)_Part 11: Guidance on Usability, ISO 9241-11:1998.

International Organization for Standardization, 1998. [143]

International Organization for Standardization. Ergonomic Requirements for Work with

Visual Displays Based on Flat Panels), ISO 13406:1999 (withdrawn). International

Organization for Standardization, 1999. [150]

International Organization for Standardization. Ergonomics of Human-System Interaction-Part

210: Human-Centered Design for Interactive Systems, ISO 9241-210:2010. International

Organization for Standardization, 2010. [144, 150, 151]

International Organization for Standardization. Ergonomics of Human-System Interaction-Part

302: Terminology for Electronic Visual Displays, ISO 9241-302:2008. International

Organization for Standardization, 2008. [150]

International Organization for Standardization. Ergonomics of Human-System Interaction-

Part 305: Optical Laboratory Test Methods for Electronic Visual Displays, ISO 9241-

305:2008. International Organization for Standardization, 2008. [150]

International Organization for Standardization. General Requirements for the Competence of

Testing and Calibration Laboratories, ISO/IEC 17025:2005(E), 2d ed. International

Organization for Standardization, 2005. [99, 183, 186]

International Organization for Standardization. Human-Centered Design Process for Interactive

Systems, ISO 13407:1999. Withdrawn, 1999. Revised by International Organization for

Standardization. Ergonomics of Human-System Interaction-Part 210: Human-Centred

Design for Interactive Systems, ISO 9241-210:2010. International Organization for

Standardization, 2010. [144, 146, 150]

International Organization for Standardization. Information Technology-Software Product

Evaluation, ISO/IEC 14598:1999. International Organization for Standardization, 1999.

[151]

International Organization for Standardization. Information Technology-Software User

Documentation Process, ISO/IEC 15910:1999 (withdrawn). International Organization

for Standardization, 1999. [151]

International Organization for Standardization. Information Technology-User System Interfaces

and Symbols-Icon Symbols and Functions, ISO/IEC 11581:2000. International

Organization for Standardization, 2000. [152]

International Organization for Standardization. Lighting of Work Places, ISO 8995:2002.

International Organization for Standardization, 2002. [153]

International Organization for Standardization. Software Engineering-Product Quality, ISO/

IEC 9126:2001. International Organization for Standardization, 2001. [150, 151]

International Organization for Standardization. Software Engineering-Software Product Quality

Requirements and Evaluation (SQuaRE) —CCommon Industry Format (CIF) for Usability

Test Reports, ISO/IEC 25062:2006. International Organization for Standardization, 2006.

[150]

219 Latent Print Examination and Human Factors: Improving the Practice through a Systems Approach

The Report of the Expert Working Group on Human Factors in Latent Print Analysis 
International Organization for Standardization. Systems and Software EngineeringRequirements for Acquirers and Suppliers of User Documentation, ISO/IEC/IEEE 26512:2011. International Organization for Standardization, 2001. [151]

Interpol European Expert Group on Fingerprint Identification - IEEGFI II. Method for Fingerprint Identification. Interpol European Expert Group on Fingerprint Identification - IEEGFI II, 2004. [47, 51]

Isaac, D., M. Avila, and A. Cialdini. "Comparison of the Original Amsler Grid with the Preferential Hyperacuity Perimeter for Detecting Choroidal Neovascularization in AgeRelated Macular Degeneration." Optometry, 79 (2008): 397. [148]

Jain, A., Y. Chen, and M. Demirkus. "Pores and Ridges: High-Resolution Fingerprint Matching Using Level 3 Features." IEEE Transactions on Pattern Analysis and Machine Intelligence, 29, no. 1 (2007): 15-27. [46, 62]

Jefferys, H. Theory of Probability, 3d ed. Oxford University Press, 1961. [134]

Jorgenson, J. Mentoring Programs: An Overview. National Academy of Public Administration, 1992. [166]

Juslen, H. and A. Tenner. "Mechanisms Involved in Enhancing Human Performance by Changing the Lighting in The Industrial Workplace." International Journal of Industrial Ergonomics, 35 (2005): 843. [153]

Kahneman, D. and A. Tversky. "Subjective Probability: A Judgment of Representativeness." Cognitive Psychology, 3 (July 1972): 430. [10]

Kaplan, R. and S. Kaplan. The Experience of Nature: A Psychological Perspective. Cambridge University Press, 1989. [153]

Kaye, D. "Identification, Individualization and Uniqueness: What's the Difference?" Law, Probability \& Risk, 8 (2009): 85. [14, 18, 130]

Kaye, D. "Interpretation: A Legal Perspective." In Wiley Encyclopedia of Forensic Sciences edited by A. Moenssens and A. Jamieson, vol. 3, pp. 1561-65. J. Wiley \& Sons, 2009. [128]

Kaye, D. "Probability, Individualization, and Uniqueness in Forensic Science Evidence: Listening to the Academies." Brooklyn Law Review, 75 (2010): 1163. [15, 31, 132]

Kaye, D. "Questioning a Courtroom Proof of the Uniqueness of Fingerprints." International Statistical Review, 71 (2003): 521. [15]

Kaye, D. "Rounding Up the Usual Suspects: A Legal and Logical Analysis of DNA Database Trawls." North Carolina Law Review, 87, no. 2 (2009): 425-503. [71, 97]

Kaye, D. The Double Helix and the Law of Evidence. Harvard University Press, 2010. [119, 126, $132,135]$

Kaye, D. "The NRC Bullet-Lead Report: Should Science Committees Make Legal Findings?" Jurimetrics Journal of Law, Science, and Technology, 46 (2005): 91. [137]

Kaye, D. "Trawling DNA Databases for Partial Matches: What Is the FBI Afraid Of?" Cornell Journal of Law and Public Policy, 19 (2009): 145. [85]

Kaye, D., D. Bernstein, and J. Mnookin. The New Wigmore, A Treatise on Evidence: Expert Evidence. 2nd ed. Aspen Publishers, 2011. [14, 18, 23, 25, 69, 70, 92, 93, 115, 116, 120 , $121,123,127,129,135,136,137]$

Kaye, D. and D. Freedman. "Reference Guide on Statistics." In Reference Manual on Scientific Evidence edited by Committee on the Development of the Third Edition of the Reference Manual on Scientific Evidence and Committee on Science, Technology, and Law Policy and Global Affairs, 3d edition, pp. 211 - 302. National Academies Press, 2011. [119] 
Kaye, D., V. Hans, B. Dann, et al. "Statistics in the Jury Box: How Jurors Respond to Mitochondrial DNA Probabilities.” Journal of Empirical Legal Studies, 4 (2007): 797. [135]

Kaye, D. and G. Sensabaugh. "Reference Guide on DNA Evidence." Reference Manual on Scientific Evidence. 3rd ed. National Academies Press, 2000. [131]

Kent, R. and M. Chial. "Talker Identification: Scientific Status." In Modern Scientific Evidence: The Law and Science of Expert Testimony edited by Faigman, D., M. Saks, J. Sanders, et al., vol. 1, pp. 113 - 151. Thomson Reuters/West, 2009. [125]

Kersta, L. "The Law: Speak, Voiceprint." Time. January 10, 1972. [31]

Kingston, C. "Probabilistic Analysis of Partial Fingerprint Patterns." Doctorate of Criminology diss., University of California, Berkeley, 1964. [61]

Kirkpatrick, D. and J. Kirkpatrick. Evaluating Training Programs: The Four Levels. 3rd ed. Berrett-Koehler Publishers, 2006. [165]

Koehler, J. "Fingerprint Error Rates And Proficiency Tests: What They Are And Why They Matter." Hastings Law Journal, 59 (2008): 1077. [29, 33, 125, 126]

Kolb, D. Experiential Learning: Experience as the Source of Learning and Development. Prentice Hall, 1984. [151]

Kolich, M. and S. Taboun. "Ergonomics Modeling and Evaluation of Automobile Seat Comfort." Ergonomics, 47, no. 8 (June 22, 2004): 841-63. [149]

Kon, C. and D. De Alwis. “A New Colour Vision Test for Clinical Use.” Eye (London, England), 10, pt. 1 (1996): 65-74. [148]

Kram, K. and M. Bragar. "Career Development Through Mentoring: A Strategic Approach for the 1990s - Part I.” Mentoring International, 5, no. 3 (Summer 1991): 3-13. [166]

Krane, D., S. Ford, J. Gilder, et al. "Sequential Unmasking: A Means of Minimizing Observer Effects in Forensic DNA Interpretation." Journal of Forensic Sciences, 53 (2008): 1006. $[12,44]$

Kryszczuk, K., A. Drygajlo, and P. Morier. "Extraction of Level 2 and Level 3 Features for Fragmentary Fingerprint Comparison." In Proceedings of the Second COST Action 275 Workshop, 2004, 83-88. [46]

Kryszczuk, K., A. Drygajlo, and P. Morier. "Study of the Distinctiveness of Level 2 and Level 3 Features in Fragmentary Fingerprint Comparison." Presented at the Proceedings of Biometric Authentication Workshop, ECCV, Prague, Czech Republic 2004, 124-33. [62]

Kumbnani, H. "Dermatoglyphics: A Review." In Anthropology Today: Trends, Scope and Applications, edited by M. Bhasin and V. Bhasin, 285-95. Kamla-Raj Enterprises, 2007. [56]

Kumho Tire Co. v. Carmichael, 526 U.S. 137, 141, 154 (1999). [121]

Kwan, Q. "Inference of Identity of Source." PhD thesis of Criminology, University of California, Berkeley, 1977. [68]

Kyles v. Whitley, 514 U.S. 419, 437 (1995). [91]

Landrigan, C., G. Parry, C. Bones, et al. "Temporal Trends in Rates of Patient Harm Resulting from Medical Care.” New England Journal of Medicine, 363 (2010): 2124. [22]

Langenburg, G. "A Performance Study of the ACE-V Process: A Pilot Study to Measure the Accuracy, Precision, Reproducibility, Repeatability, and Biasability of Conclusions Resulting from the ACE-V Process." Journal of Forensic Identification, 59, no. 2 (2009): 219-57. [75, 125]

Langenburg, G. "Deposition of Bloody Friction Ridge Impressions." Journal of Forensic Identification, 58, no. 3 (2008): 355-389. [66]

221 Latent Print Examination and Human Factors: Improving the Practice through a Systems Approach The Report of the Expert Working Group on Human Factors in Latent Print Analysis 
Langenburg, G. "Pilot Study: A Statistical Analysis of the ACE-V Methodology-Analysis Stage." Journal of Forensic Identification, 54 (2004): 64-79. [49]

Langenburg, G. "Scientific Research in the Forensic Discipline of Friction Ridge Individualization.” In The Fingerprint Sourcebook. National Institute of Justice, 2011. [83]

Langenburg, G. and C. Champod. "The GYRO System-A Recommended Approach to More Transparent Documentation." Journal of Forensic Identification, 61, issue 4 (July/August 2011): 373 - 384. [45, 48, 49, 51]

Langenburg, G., C. Champod, T. Genessay, et al. "Informing the Judgments of Fingerprint Analysts Using Quality Metric and Statistical Assessment Tools.” National Institute of Justice Impression and Pattern Evidence Symposium, Clearwater, Florida. August 2010. $[49,52]$

Langenburg, G., C. Champod, and P. Wertheim. "Testing for Potential Contextual Bias Effects During the Verification Stage of the ACE-V Methodology When Conducting Fingerprint Comparisons." Journal of Forensic Sciences, 54, no. 3 (May 2009): 571-82. [12, 50, 52]

Leat, S. and G. Woo. "The Validity of Current Clinical Tests of Contrast Sensitivity and Their Ability to Predict Reading Speed in Low Vision.” Eye (London, England), 11, pt. 6 (1997): 893. [148]

Lee, H. and R. Gaensslen, ed. Advances in Fingerprint Technology. 2nd ed. CRC Press, 2001. [167]

Lee, L., S. Frederick, and D. Ariely. "Try It, You'll Like It: The Influence of Expectation, Consumption, and Revelation on Preferences for Beer." Psychological Science, 17 (2006): 1054. [11]

Leslie, R. "Capturing the Daylight Dividend in Buildings: Why and How?" Building and Environment, 38, no. 2 (February 2003): 381-85. [153]

Leveson, B. Expert Evidence in Criminal Courts-The Problem, Address to the Forensic Science Society, Kings College, University of London, November 18, 2010. [vi]

Levine, R. Ethics and Regulation of Clinical Research. 2nd ed. Yale University Press, 1986. [11]

Lewicki v. Longshore, No. 17004/06, 2009 WL 4667411, at 2 (N.Y. Sup. Ct. Dec. 7, 2009). [119]

Lichtenstein, S., B. Fischhoff, and L. Phillips. "Calibration of Probabilities: The State of the Art to 1980." Judgment Under Uncertainty: Heuristics and Biases. Cambridge University Press, 1982. [23]

Lin, C., J. Liu, J. Osterburg, et al. "Fingerprint Comparison I: Similarity of Fingerprints." Journal of Forensic Science, 27, no. 2 (1982): 290. [60]

Locard, E. "La Preuve Judiciaire par les Empreintes Digitales." Archives d'Anthropologie Criminelle, de Médecine Légale et de Psychologie Normale et Pathologique, 29 (1914): 321-48. [55, 204]

Louis, T. "Confidence Intervals for a Binomial Parameter After Observing No Successes." The American Statistician, 35 (1981): 154. [34]

Lubet, S. "Expert Witnesses: Ethics and Professionalism." Georgetown Journal of Legal Ethics, 12 (1999): 465. [116]

Lucas, D. "The Ethical Responsibilities of the Forensic Scientist: Exploring the Limits." Journal of Forensic Sciences, 34 (1989): 724. [92]

Luthar, S., D. Cicchetti, B. Becker. "Research on Resilience: Response to Commentaries." Child Development, 71, no. 3 (2003): 573. [140]

Maceo, A. "Qualitative Assessment of Skin Deformation: A Pilot Study." Journal of Forensic Identification, 59, no. 4 (2009): 390-440. [46, 65, 66] 
Macklis, R., T. Meier, and M. Weinhous. "Error Rates in Clinical Radiotherapy." Journal of Clinical Oncology, 16 (1998): 551. [22]

Maguire, M. "Context of Use Within Usability Activities." International Journal of HumanComputer Studies, 55 (2001): 453-83. [143, 146]

Massachusetts General Laws, ch. 111, §13. [92]

McClelland, J. and D. Rumelhart. "An Interactive Activation Model of Context Effects in Letter Perception: Part 1, An Account of Basic Findings.” Psychological Review, 88 (September 1981): 375. [11]

McCormick, C. McCormick on Evidence. Edited by Kenneth Broun. 6th ed. Thomson/West, 2006. [113, 115, 123, 129,]

Melendez Diaz v. Massachusetts, 129 S. Ct. 2527, 2535 (2009). [93]

Melsa, J. and D. Cohn. Decision and Estimation Theory. McGraw-Hill, 1978. [26]

Meshkati, N. "Human Factors in Large-Scale Technological Systems' Accidents: Three-Mile Island, Bhopal, Chernobyl." Organization \& Environment, 5, no. 2 (June 1991): 133-54. [142]

Microsoft Learning Whitepaper. The Value of Certification: Connecting the Dots Between Employers and Employees. Microsoft Corporation, March 2007. [171]

Midena, E., A. Degli, M. Blarzino, et al. "Macular Function Impairment in Eyes with Early Age-Related Macular Degeneration." Investigative Ophthalmology \& Visual Science, 38 (February 1997): 469. [148]

Miller, L. "Procedural Bias in Forensic Science Examinations of Human Hair." Law and Human Behavior, 11, no. 2 (June 1987): 157. [10]

Mnookin, J. "Fingerprint Evidence in an Age of DNA Profiling." Brooklyn Law Review, 67 (2001): 13. [vi, 113]

Mnookin, J. "The Courts, the NAS, and the Future of Forensic Science." Brooklyn Law Review, 75, no. 4 (2010): 1209. [39, 69, 137]

Mnookin, J. "The Validity of Latent Fingerprint Identification: Confessions of a Fingerprinting Moderate." Law, Probability \& Risk, 7 (2008): 127. [114]

Mnookin, J., S. Cole, I. Dror, et al. "The Need for a Research Culture in the Forensic Sciences." UCLA Law Review, 58 (2011): 725. [168]

Moallem, A. "Usability of Software Online Documentation: A User Study." HCI International, 1 (2003): 549. [151]

Montés-Micó, R. and T. Ferrer-Blasco. "Contrast Sensitivity Loss in the Peripheral Visual Field Following Laser in Situ Keratomileusis." Journal of Cataract and Refractive Surgery, 33 (2007): 1120. [148]

Morrison, G. "Measuring the Validity and Reliability of Forensic Likelihood-Ratio Systems." Science \& Justice, 51 (2011): 91 - 98. [135]

Morrison, W., E. Haas, D. Shaffner, et al. "Noise, Stress, and Annoyance in a Pediatric Intensive Care Unit." Critical Care Medicine, 31, no. 1 (2003): 113. [153]

Moses, K. “Anatomy of Error." Presented at International Association for Identification 89th International Educational Conference, St. Louis, Missouri. August 2004. [12]

Moses, K. "Automated Fingerprint Identification System (AFIS).” The Fingerprint Sourcebook. National Institute of Justice, 2011. [77, 79]

Muzammil, M., A. Khan, F. Hasan, et al. "Effect of Noise on Human Performance Under Variable Load in a Die Casting Industry - A Case Study.” Journal of Environmental Science Engineering, 46 (2004): 49. [154]

223 Latent Print Examination and Human Factors: Improving the Practice through a Systems Approach The Report of the Expert Working Group on Human Factors in Latent Print Analysis 
Nance, D. and S. Morris. "Juror Understanding of DNA Evidence: An Empirical Assessment of Presentation Formats for Trace Evidence with a Relatively Small Random-Match Probability." Journal of Legal Studies, 34 (2005): 395. [135]

National Academy of Sciences, Institute of Medicine, Board on Health Care Services. Keeping Patients Safe: Transforming the Work Environment for Nurses. National Academies Press, 2004. [154]

National Academy of Sciences, Institute of Medicine, Committee on Quality of Health Care in America. To Err Is Human: Building A Safer Health System. National Academies Press, 1999. [vi, 21, 140]

National Academy of Sciences, National Research Council, Committee on Behavioral and Social Science Research to Improve Intelligence Analysis for National Security. Intelligence Analysis for Tomorrow: Advances from the Behavioral and Social Sciences. National Academies Press, 2011. [23]

National Academy of Sciences, National Research Council, Committee on DNA Forensic Science: An Update. The Evaluation of Forensic DNA Evidence. National Academies Press, 1996. [15, 17, 34, 127, 131]

National Academy of Sciences, National Research Council, Committee on DNA Technology in Forensic Science. DNA Technology in Forensic Science. National Academies Press, 1992. $[31,126]$

National Academy of Sciences, National Research Council, Committee on Evaluation of Sound Spectrograms. On the Theory and Practice of Voice Identification. National Academies Press, 1979. [16, 26, 31]

National Academy of Sciences, National Research Council, Committee on Identifying the Needs of the Forensic Science Community. Strengthening Forensic Science in the United States: A Path Forward. National Academies Press, 2009. [9, 12, 15, 16, 18, 21, 32, 79, 94, 96, 99, 113, 116, 123, 124, 128, 130, 133, 165]

National Academy of Sciences, National Research Council, Committee on Scientific Assessment of Bullet Lead Elemental Composition Comparison. Forensic Analysis: Weighing Bullet Lead Evidence. National Academies Press, 2004. [94, 136, 137]

National Academy of Sciences, National Research Council, Committee to Review the Scientific Evidence on the Polygraph. The Polygraph and Lie Detection. National Academies Press, 2003. [125]

National Conference of State Legislatures. Strengthening Forensic Science Oversight. National Conference of State Legislatures, February 2010. [170]

National Institute of Justice and Scientific Working Group on Friction Ridge Analysis, Study and Technology. The Fingerprint Sourcebook. National Institute of Justice, August 2011. [vi, $11,77,83,167]$

Neumann, C., C. Champod, R. Puch-Solis, et al. "Computation of Likelihood Ratios in Fingerprint Identification for Configurations of Any Number of Minutiae." Journal of Forensic Sciences, 52, no. 1 (2007): 54-64. [46, 61, 84, 87]

Neumann, C., C. Champod, R. Puch-Solis, et al. "Computation of Likelihood Ratios in Fingerprint Identification for Configurations of Three Minutiae." Journal of Forensic Sciences, 51 (2006): 1255. [84, 87]

Neumann, C., I. Evett, and J. Skerrett. "Quantifying the Weight of Evidence from a Forensic Fingerprint Comparison: A New Paradigm.” Journal of the Royal Statistical Society, Series A, forthcoming. [87] 
Nevid, J. "Effects of Brand Labeling on Ratings of Product Quality." Perceptual and Motor Skills, 53 (1981): 407. [11]

Nickerson, R. "Confirmation Bias: A Ubiquitous Phenomenon in Many Guises." Review of General Psychology, 2 (1998): 175. [10,11]

Nielsen, J. Usability Engineering. Morgan Kaufmann, 1993. [143, 146, 158]

Nill, N. "IQF (Image Quality of Fingerprint) Software Application.” MITRE (May 2007). Accessed April 22, 2011. http://www.mitre.org/tech/mtf/. [48]

Noweir, M. "Noise Exposure as Related to Productivity, Disciplinary Actions, Absenteeism, and Accidents Among Textile Workers.” Journal of Safety Research, 15 (1984): 163. [154]

Office of Management and Budget. Final Information Quality Bulletin for Peer Review. Office of Management and Budget, December 15, 2004. [vii]

Olaosum, A., O. Ogundiran, and J. Tobih. "Health Hazards of Noise: A Review Article." Research Journal of Medical Sciences, 3 (2009): 115. [154]

Osterburg, J. "An Inquiry into the Nature of Proof. The Identity of Fingerprints." Journal of Forensic Sciences, 9, no. 4 (1964): 413-27. [48, 55, 205]

Osterburg, J., T. Parthasarathy, T. Raghavan, et al. "Development of a Mathematical Formula for the Calculation of Fingerprint Probabilities Based on Individual Characteristics.” Journal of the American Statistical Association, 72 (1977): 772. [60]

Page, M., J. Taylor, and M. Blenkin. "Forensic Identification Science Evidence Since Daubert: Part II-Judicial Reasoning in Decisions to Exclude Forensic Identification Evidence on Grounds of Reliability.” Journal of Forensic Sciences, 56 (2011): 913 - 917. [130]

Page, M., J. Taylor, and M. Blenkin. "Uniqueness in the Forensic Identification Sciences_Fact or Fiction?” Forensic Science International, 206 (2011): 12, 1415. [17]

Palmer, S. "The Effects of Contextual Scenes on the Identification of Objects." Memory and Cognition, 3 (1975): 519. [11]

Pankanti, S., S. Prabhakar, and A. Jain. "On the Individuality of Fingerprints." IEEE

Transactions on Pattern Analysis and Machine Intelligence, 24, no. 8 (2002): 1010. [83, 132]

Parmigiani, G. Modeling in Medical Decision Making: A Bayesian Approach. Wiley, 2002. [28]

Parsons, N., J. Smith, E. Thönnes, et al. "Rotationally Invariant Statistics for Examining the Evidence from the Pores in Fingerprints." Law Probability \& Risk, 7, no. 1 (2007): 1. [61]

Pendergrass v. State, 913 N.E.2d 703, 709 (Ind. 2009). [92]

People v. Clark, 857 P.2d 1099 (Cal. 1993). [123]

People v. Cua, 119 Cal.Rptr.3d 391 (Cal. Ct. App. 2011). [131]

People v. McClanahan, 729 N.E.2d 470, 472 (Ill. 2000). [92]

People v. McDaniel, 670 N.W.2d 659 (Mich. 2003). [92]

People v. McWhorter, 212 P.3d 692 (Cal. 2009). [81]

People v. Williams, 939 N.E. 2d 268, 270 (Ill. 2010), cert. granted sub nom. Williams v. Illinois, 80 U.S.L.W. 3003 (U.S. June 28, 2011). [93]

Perlin, M., J. Kadane, and R. Cotton. "Match Likelihood Ratio for Uncertain Genotypes." Law, Probability \& Risk, 8 (2009): 289. [87]

Peterson, J., G. Lin, M. Ho, et al. "The Feasibility of External Blind DNA Proficiency Testing." Journal of Forensic Sciences, 48 (2003): 21. [33]

Peterson, P., C. Dreyfus, M. Gische, et al. "Latent Prints: A Perspective on the State of the Science." Forensic Science Communications, 11, no. 4 (October 2009). [14, 16, 95, 99, $122,126,129,139]$ 
Pierce v. Gilchrist, 359 F.3d 1279 (10th Cir. 2004). [91]

Potchen, E., J. Gard, P. Lazar, et al. "The Effect of Clinical History Data on Chest Film Interpretation: Direction or Distraction?" Investigative Radiology, 14 (1979): 404. [11, 40]

Powers, M. "Paper Tools for Assessing Visual Function." Optometry \& Vision Science, 86 (2009): 613. [148]

Privacy Act of 1974, 5 U.S.C. $§ 552 \mathrm{a}(\mathrm{b})(5)$. [85]

Pronin, E. "Perception and Misperception of Bias in Human Judgment." Trends in Cognitive Science, 11 (January 2007): 37. [11]

R. v. Smith, 2011 EWCA Crim 1296. [vi]

Rand, S., M. Schürenkamp, and B. Brinkmann. "The GEDNAP (German DNA Profiling Group) Blind Trial Concept.” International Journal of Legal Medicine, 116, no. 4 (2002): 199. $[33,36]$

Rand, S., M. Schürenkamp, and B. Brinkmann. "The GEDNAP (German DNA Profiling Group) Blind Trial Concept Part II: Trends and Developments.” International Journal of Legal Medicine, 118, no.2 (2004): 83. [36]

Ratha, N., S. Chen, and A. Jain. "Adaptive Flow Orientation-Based Feature Extraction in Fingerprint Images.” Pattern Recognition, 28 (1995): 1657. [25]

Reason, J. Human Error. Cambridge University Press, 1990. [23, 174, 175]

Rettig, M. "Nobody Reads Documentation." Communications of the ACM, 34 (1991): 19. [151]

Richmond, S. Do Fingerprint Ridges and Characteristics Within Ridges Change with Pressure? Australian Federal Police, Forensic Services, 2004. [5, 46, 47]

Risinger, D., M. Saks, W. Thompson, et al. "The Daubert/Kumho Implications of Observer Effects in Forensic Science: Hidden Problems of Expectation and Suggestion." California Law Review, 90, no. 1 (January 2002). [10, 44]

Roberts, K. "Some Characteristics of One Type of High Reliability Organization." Organization Science, 1, no. 2 (1990): 160. [181]

Roberts, K. and R. Bea. "Must Accidents Happen? Lessons from High-Reliability Organizations." Academy of Management Executive, 15, no. 3 (2001): 70. [181]

Robertson, B. and G. Vignaux. Interpreting Evidence: Evaluating Forensic Science in the Courtroom. John Wiley \& Sons, 1995. [19, 133]

Rochlin, G. "Defining 'High Reliability' Organizations in Practice: A Taxonomic Prologue.” In New Challenges to Understanding Organizations. Macmillan, 1993. [181]

Roddy, A. and J. Stosz. "Fingerprint Features - Statistical Analysis and System Performance Estimates." Proceedings of the IEEE, 85, no. 9 (1997): 1390-1421. [5, 46, 61]

Rosekind, M., P. Gander, L. Cornell, et al. Crew Factors in Flight Operations X: Alertness Management in Flight Operations Education Module. National Aeronautics and Space Administration, 2001. [154]

Rothman, K., S. Greenland, and T. Lash. Modern Epidemiology. 3d ed. Lippincott, Williams, \& Wilkins, 2008. [13, 25]

Royal Canadian Mounted Police. Fingerprint Manual. Chapter 2. Royal Canadian Mounted Police, 1990. [56, 83]

RTI International. "Forensic Science Education: Web-Based Professional Development and Continuing Education for Forensic Scientists.” Accessed November 29, 2011. http://www.rti.org/page.cfm/Forensic_Science_Education. [170]

Saferstein, R. Criminalistics: An Introduction to Forensic Science. Prentice Hall, 2000. [167] 
Saks, M. "Merlin and Solomon: Lessons From the Law's Formative Encounters with Forensic Identification Science.” Hastings Law Journal, 49 (1998): 1102. [33]

Saks, M. and J. Koehler. "The Individualization Fallacy in Forensic Science Evidence." Vanderbilt Law Review, 61 (2008): 199. [15]

Saks, M., D. Risinger, R. Rosenthal, et al. "Context Effects in Forensic Science: A Review and Application of the Science of Science to Crime Laboratory Practice in the United States." Science and Justice, 43, no. 2 (April - June 2003): 77. [10]

Sanders, J. and E. McCormick. Human Factors Engineering and Design. McGraw-Hill Science/ Engineering/Math, 1993. [vi, 142]

Santamaria Beltran, F. “A New Method for Evaluating Ridge Characteristics.” Fingerprint and Identification Magazine, 36, no.11 (1955). [61]

Santamaria Beltran, F. "Sous-Commission á la Dactyloscopie, Nouveaux Concepts d'Évaluation des Points Caractéristiques de F. Santamaria Beltran." Assemblée Générale de la Commission International de Police Criminelle - Oslo. 25 June 1953. [60]

Santamaria Beltran, F. "Une Nouvelle Méthode d'Évaluation des Points Caracteristiques des Crêtes Papillaires." Assemblée Générale de la Commission International de Police Criminelle - Oslo. 24-29 June 1953. [60]

Saviers, K. "Friction Skin Characteristics: A Study and Comparison of Proposed Standards." Journal of Forensic Identification, 39 (1989): 157. [61]

Schreiber, M. "The Clinical History as a Factor in Roentgenogram Interpretation." The Journal of the American Medical Association, 185 (1963): 137. [11]

Scientific Working Group for Firearms and Toolmarks. Guidelines for the Standardization of Comparison Documentation. Scientific Working Group for Firearms and Toolmarks, October 2010. [91]

Scientific Working Group for the Analysis of Seized Drugs. Scientific Working Group for the Analysis of Seized Drugs (SWGDRUG) Recommendations. Revision 5.1. Scientific Working Group for the Analysis of Seized Drugs, January 2011. [91, 96]

Scientific Working Group for the Analysis of Seized Drugs. Supplemental Document SD-1 for Part I, A Code of Professional Practice for Drug Analysts. Scientific Working Group for the Analysis of Seized Drugs, October 2004. [94 (Recommendation 2.3.1), 96 (Recommendation 2.3.1), 97 (Recommendation 2.3.1), 100 (Recommendation 3.2.1)]

Scientific Working Group on Friction Ridge Analysis, Study and Technology. A Model Policy for Friction Ridge Examiner Professional Conduct. Version 1.0. Scientific Working Group on Friction Ridge Analysis, Study and Technology, December 2008. [117, 200]

Scientific Working Group on Friction Ridge Analysis, Study and Technology. Draft for Comment: Standard for the Definition and Measurement of Rates of Errors and Inappropriate Decisions in Friction Ridge Examination. Version 1.0. Scientific Working Group on Friction Ridge Analysis, Study and Technology, February 2011. [29]

Scientific Working Group on Friction Ridge Analysis, Study and Technology. Draft for Comment: Standards for Examining Friction Ridge Impressions and Resulting Conclusions (To Replace: Friction Ridge Examination Methodology for Latent Print Examiners, Version 1.01, and Standards for Conclusions, Version 1.0). Version 1.0. Scientific Working Group on Friction Ridge Analysis, Study and Technology, August 2010. $[17,72,100]$

Scientific Working Group on Friction Ridge Analysis, Study and Technology. Glossary, Version 2.0. Scientific Working Group on Friction Ridge Analysis, Study and Technology, May 2009. [123]

227 Latent Print Examination and Human Factors: Improving the Practice through a Systems Approach The Report of the Expert Working Group on Human Factors in Latent Print Analysis 
Scientific Working Group on Friction Ridge Analysis, Study and Technology. Quality Assurance Guidelines for Latent Print Examiners. Version 3.0. Scientific Working Group on Friction Ridge Analysis, Study and Technology, September 2006. [139 (Guideline 5.5.1), 184]

Scientific Working Group on Friction Ridge Analysis, Study and Technology. Standard for Friction Ridge Automation Training (Latent/Tenprint). Version 1.0. Scientific Working Group on Friction Ridge Analysis, Study and Technology, May 2009. [169]

Scientific Working Group on Friction Ridge Analysis, Study and Technology. Standard for the Application of Blind Verification of Friction Ridge Examinations. Version 1.0. Scientific Working Group on Friction Ridge Analysis, Study and Technology, February 2011. [185]

Scientific Working Group on Friction Ridge Analysis, Study and Technology. Standard for the Documentation of Analysis, Comparison, Evaluation, and Verification (ACE-V) (Latent). Version 1.0. Scientific Working Group on Friction Ridge Analysis, Study and Technology, February 2010. [91, 97, 98, 99]

Scientific Working Group on Friction Ridge Analysis, Study and Technology. Standard Terminology of Friction Ridge Examination (To Replace: Glossary, Version 2.0). Version 3. Scientific Working Group on Friction Ridge Analysis, Study and Technology, February 11, 2011. [14, 17, 100]

Scientific Working Group on Friction Ridge Analysis, Study and Technology. Standards for Conclusions. Version 1.0. Scientific Working Group on Friction Ridge Analysis, Study and Technology, September 2003. [14, 96, 100]

Scientific Working Group on Friction Ridge Analysis, Study and Technology. Standards for the Minimum Qualifications and Training to Competency for Friction Ridge Examiner Trainees (Latent/Tenprint). Version 1.0. Scientific Working Group on Friction Ridge Analysis, Study and Technology, February 2010. [164, 169]

Scientific Working Group on Friction Ridge Analysis, Study and Technology. Strengthening Forensic Science in the United States: A Path Forward, Position Statement. Scientific Working Group on Friction Ridge Analysis, Study and Technology, 2009. [12, 16, 32, 35, 90, 97, 164]

Scientific Working Group on Materials Analysis. Forensic Human Hair Examination Guidelines. Scientific Working Group on Materials Analysis, April 2005. [96]

Sclove, S. "The Occurrence of Fingerprint Characteristics as a Two-Dimensional Poisson Process." Communications in Statistics - Theoretical Methods, A9 (1980): 675. [61]

Sclove, S. "The Occurrence of Fingerprint Characteristics as a Two-Dimensional Process." Journal of the American Statistical Association, 74 (1979): 588. [61]

SeaCrest Company. "The Value of Certification." Credentialing Talk (August 1, 2007). Accessed October 21, 2011. http://seacrestcompany.blogspot.com/2007/08/value-ofaccreditation.html. [171]

Senders, J. and N. Moray. Human Error: Cause, Prediction, and Reduction. Psychology Press, 1991. [176]

Seppänen, O., W. Fisk, and Q. Lei. Effect of Temperatures on Task Performance in Office Environment. Helsinki Univ. of Technology, Laboratory of Heating, Ventilating, and AirConditioning, July 2006. [152]

Shrivastava, P., I. Mitroff, D. Miller, et al. "Understanding Industrial Crises.” Journal of Management Studies, 25, no. 4 (1988): 285. [140]

Shultz, K. and D. Grimes. "Blinding in Randomized Trials: Hiding Who Got What." Lancet, 359 (February 2002): 696. [11] 
Sowden, P., I. Davies, and P. Roling. "Perceptual Learning of the Detection of Features in X-Ray Images: A Functional Role for Improvements in Adults' Visual Sensitivity?" Journal of Experimental Psychology: Human Perception and Performance, 26, no. 1 (2000): 37990. [66]

Srihari, S., C. Huang, and H. Srinivasan. "On the Discriminability of the Handwriting of Twins." Journal of Forensic Science, 53 (2008): 430. [135]

Srihari, S., H. Srinivasan, and G. Fang. "Discriminability of Fingerprints of Twins." Journal of Forensic Identification, 58, no. 1 (2008): 109-27. [46]

Stacey, R. "A Report on the Erroneous Fingerprint Individualization in the Madrid Train Bombing Case.” Journal of Forensic Identification, 54, no. 6 (2004): 706. [12, 50]

State v. Foreman, 954 A.2d 135 (Conn. 2008). [123]

State v. Hartman, 754 N.E.2d 1150 (Ohio 2001). [81]

State v. McGrew, 682 N.E.2d 1289 (Ind. 1997). [136]

State v. Proctor, 595 S.E.2d 480 (S. Car. 2004). [127]

State v. Reid, 757 A.2d 482, 487 (Conn. 2000). [123]

State v. Rose, No. K06-0545 (Md. Cir. Ct. Oct. 19, 2007). [130]

State v. Swinton, 847 A.2d 921 (Conn. 2004). [81]

Stevenson, K., S. Gibson, D. MacDonald, et al. "Measurement of Process as Quality Control in the Management of Acute Surgical Emergencies." British Journal of Surgery, 95 (2007): 376. [24]

Stone, R. and R. McCloy. "Ergonomics in Medicine and Surgery.” BMJ, 328, no. 7448 (May 6, 2004). [150]

Stoney, D. “A Medical Model for Criminalistics Education.” Journal of Forensic Sciences, 33 (1988): 1086-94. [169]

Stoney, D. “A Quantitative Assessment of Fingerprint Individuality." PhD thesis, University of California, Davis, 1985. [61]

Stoney, D. "Measurement of Fingerprint Individuality." In Advances in Fingerprint Technology, edited by Henry C. Lee and R.E. Gaensslen, 327-87. 2nd ed. CRC Press, 2001. [14, 58, $83,124,132,137]$

Stoney, D. "What Made Us Ever Think We Could Individualize Using Statistics?" Journal of the Forensic Science Society, 31 (1991): 197. [15]

$\mathrm{Su}, \mathrm{C}$. and S. Srihari. "Evaluation of Rarity of Fingerprints in Forensics." In Proceedings of Neural Information Processing Systems Conference, Vancouver, Canada, December 2010. [83, 84]

Su, C. and S. Srihari. "Generative Models for Fingerprint Individuality Using Ridge Models." In Proceedings 19th International Conference on Pattern Recognition, Tampa, Florida, December 8-11, 2008. [84]

$\mathrm{Su}, \mathrm{C}$. and S. Srihari. "Probability of Random Correspondence for Fingerprints." Computation Forensics: Lecture Notes in Computer Science, 5718 (2009): 55. [61]

Sunness, J., G. Rubin, C. Applegate, et al. "Visual Function Abnormalities and Prognosis in Eyes with Age-Related Geographic Atrophy of the Macula and Good Visual Acuity." Ophthalmology, 104 (1997): 1677. [148]

Sunness, J., G. Rubin, A. Broman, et al. "Low Luminance Visual Dysfunction as a Predictor of Subsequent Visual Acuity Loss from Geographic Atrophy in Age-Related Macular Degeneration." Ophthalmology, 115 (2008): 1480. [148]

Suppes, P. and J. Zinnes. "Basic Measurement Theory.” In Handbook of Mathematical Psychology. Wiley, 1963. [25]

229 Latent Print Examination and Human Factors: Improving the Practice through a Systems Approach The Report of the Expert Working Group on Human Factors in Latent Print Analysis 
Sweeney, C. "Lord Advocate to Appear Before Shirley McKie Fingerprint Inquiry." The Times, October 21, 2008. Accessed April 22, 2011. http://www.timesonline.co.uk/tol/news/uk/scotland/article4981867.ece. [vi]

Swets, J. and R. Pickett. Evaluation of Diagnostic Systems: Methods from Signal Detection Theory. Academic Press, 1982. [26]

Tabassi, E. and C. Wilson. "A Novel Approach to Fingerprint Image Quality." IEEE International Conference on Image Processing, 2 (September 2005): 37-40. [48]

Taherian, K. and M. Shekarchian. "Mentoring for Doctors: Do Its Benefits Outweigh Its Disadvantages?" Medical Teacher, 30 (2008): e95-e99. [167]

Tape, T. and R. Panzer. "Echocardiography, Endocarditis, and Clinical Information Bias." Journal of General Internal Medicine, 1, no.3 (1986): 300. [11]

Technical Working Group for Education and Training in Forensic Science. Education and Training in Forensic Science: A Guide for Forensic Science Laboratories, Educational Institutions, and Students. National Institute of Justice, 2004. [165, 169, 170]

Teichroeb, R. "Produce Crime Lab Error Rates, Some Urge." Seattle Post-Intelligencer, July 21, 2004. Accessed November 28, 2011. http://www.seattlepi.com/default/article/Producecrime-lab-error-rates-some-urge-1149873.php. [125]

The Public Health and Welfare, 42 U.S.C. $§ 3789 \mathrm{~g}(\mathrm{~b})$. [85]

Thomas, F. "Comments on the Discovery of Striation Matching and on Early Contributions to Forensic Firearms Identification.” Journal of Forensic Science, 12 (1967): 1 - 7. [113]

Thornton, J. "The One-Dissimilarity Doctrine in Fingerprint Identification." International Criminal Police Review, 32, no. 306 (1977): 89-95. [65]

Thornton, J. and J. Peterson. "The General Assumptions and Rationale of Forensic Identification.” Modern Scientific Evidence: The Law and Science of Expert Testimony. West Publishing Company, 1997. [13]

Tietze, S. and K. Witthuhn. Papillarleistenstruktur der menschlichen Handinnenfläche. Luchterhand, 2001. [47]

Tiller, C. "Fingerprint Identification in the United Kingdom-Do They Really Need 16 Points?" Canadian Identification Society Newsletter, 39 (1983): 11. [51]

Tippett, C., V. Emerson, F. Lawton, et al. "The Evidential Value of the Comparison of Paint Flakes from Sources Other Than Vehicles." Journal of the Forensic Science Society, 8 (1968): 6. [135]

Tiscareno v. Anderson, 639 F.3d 1016 (10th Cir. 2011), vacated in part, 421 Fed. Appx. 842 (10th Cir. 2011). [91]

Topf, M. "Hospital Noise Pollution: An Environmental Stress Model to Guide Research and Clinical Interventions." Journal of Advanced Nursing, 31 (2000): 520. [153]

Triplett, M. and L. Cooney. "The Etiology of ACE-V and Its Proper Use: An Exploration of the Relationship Between ACE-V and the Scientific Method of Hypothesis Testing." Journal of Forensic Identification, 56, no. 3 (2006): 345-55. [51]

Tversky, A. and D. Kahneman. "Rational Choice and the Framing of Decisions." Journal of Business, 59 (1986): 251. [23]

Tyler, R. Basic Principles of Curriculum and Instruction. University of Chicago Press, 1949. [167]

U.S. Department of Commerce, National Institute of Standards and Technology. Usability \& Biometrics: Ensuring Successful Biometric Systems. National Institute of Standards and Technology, June 11, 2008. [143] 
U.S. Department of Justice, National Institute of Justice. “Training.” Accessed November 29, 2011. http://www.nij.gov/training/welcome.htm. [170]

U.S. Department of Justice, Office of the Inspector General. A Review of the FBI's Handling of the Brandon Mayfield Case (Unclassified and Redacted). U.S. Department of Justice, March 2006. [vi, 12, 47, 51]

U.S. Department of Justice, Office of the Inspector General. A Review of the FBI's Progress in Responding to the Recommendations in the Office of the Inspector General Report on the Fingerprint Misidentification in the Brandon Mayfield Case. U.S. Department of Justice, 2011. [130, 185]

U.S. Department of Justice, Office of the Inspector General. The FBI DNA Laboratory: A Review of Protocol and Practice Vulnerabilities. U.S. Department of Justice, May 2004. [42]

U.S. Department of Labor, Occupational Safety \& Health Administration. OSHA Technical Manual, Section III, Chapter 2, Indoor Air Quality Investigation. Occupational Safety \& Health Administration, 1999. [152]

U.S. Department of Labor, Occupational Safety \& Health Administration. Regulations (Standards-29 CFR), Part 1910.95: Occupational Noise Exposure. Occupational Safety $\&$ Health Administration, 1991. [154]

Ulery, B., A. Hicklin, J. Buscaglia, et al. "Accuracy and Reliability of Forensic Latent Fingerprint Decisions." Proceedings of the NAS. 2011. [12, 36, 63, 125, 126]

Ulrich, R., X. Quan, C. Zimring, , et al. "The Role of the Physical Environment in the Hospital of the 21st Century: A Once-In-A-Lifetime Opportunity." In Designing the 21st Century Hospital Project, The Center for Health Design. May 2005. [142]

United States v. Baines, 573 F.3d (10th Cir. 2009). [32 (at 979, 984, 997 - 98), 123 (at 979, 992)]

United States v. Baker, 855 F.2d 1353, 1359 (8th Cir. 1988). [92]

United States v. Davis, 602 F.Supp.2d 658 (D.Md. 2009). [131]

United States v. Glynn, 578 F.Supp.2d 567, 574-75 (S.D.N.Y. 2008). [119, 120]

United States v. Gutierrez-Castro, 86 Fed. R. Evid. Serv. 319 (D.N.M. 2011). [123]

United States v. Hugh, No. Crim.A. 03-829, 2009 WL 212420 (E.D. Pa. Jan. 28 2009). [130]

United States v. Llera Plaza, Crim. No. 98-362-10, 11, 12, 2002 U.S. Dist. LEXIS 344 (E.D. Pa. Jan. 7, 2002), vacated, 188 F. Supp. 2d 549 (E.D. Pa. 2002). [32, 122, 123, 130]

United States v. McVeigh, No. 96-CR-68 (D. Colo. May 5, 1997) (transcript). [137]

United States v. Mitchell, 199 F. Supp. 2d 262 (E.D. Pa. 2002) (No. 96-407). [32]

United States v. Monteiro, 407 F.Supp.2d 351, 375 (D.Mass. 2006). [120]

United States v. Nichols, No. 96-CR-68 (D. Colo. Nov. 10, 1997) (transcript). [137]

United States v. Oates, 560 F.2d 45 (2d Cir. 1977). [92]

United States v. Taylor, 663 F.Supp.2d 1170, 1180 (D.N.M. 2009). [120, 122]

United States v. Tharpe, 38 M.J. 8, 28 (C.M.A. 1993). [116]

United States v. Willock, 696 F.Supp.2d (D.Md. 2010). [91 (at 536, 570), 119 (536, 574), 122 (536)]

United States v. Zajac, No. 2:06-cr-00811-CW (D. Utah Sept. 16, 2010). [120, 130, 137]

Vanderkolk, J. Forensic Comparative Science: Qualitative Quantitative Source Determination of Unique Impressions, Images, and Objects. Academic Press, 2009. [15, 42, 51, 137, 167]

Vanderkolk, J. "Levels of Quality and Quantity in Detail." Journal of Forensic Identification, 51, no. 5 (2001): 461-68. [68]

Vatsa, M., R. Singh, A. Noore, et al. "Combining Pores and Ridges with Minutiae for Improved Fingerprint Verification.” Signal Processing, 89, no. 12 (2009): 2676-85. [62]

231 Latent Print Examination and Human Factors: Improving the Practice through a Systems Approach The Report of the Expert Working Group on Human Factors in Latent Print Analysis 
Wargocki, P., D. Wyon, J. Sundell, et al. "The Effects of Outdoor Air Supply Rate in an Office on Perceived Air Quality, Sick Building Syndrome (SBS) Symptoms and Productivity." Indoor Air, 10, no. 4 (2000): 222-36. [152]

Warney v. Monroe County, 587 F.3d 113, 118 (2d Cir. 2009). [118]

Weingart, S., R. Wilson, R. Gibberd, et al. "Epidemiology of Medical Error." British Medical Journal, 320 (2000): 774. [22]

Wickens, T. Elementary Signal Detection Theory. Oxford University Press, USA, 2002. [26]

Wiegmann, D. and S. Shappell. A Human Error Approach to Aviation Accident Analysis: The Human Factors Analysis and Classification System. Ashgate Publishing, 2003. [23, 176]

Williams v. State, 679 A.2d 1106 (Md. 1996). [126]

Wilson, J. and N. Elman. "Organizational Benefits of Mentoring." The Executive, 4, no. 4 (1990): 88-94. [167]

Wilson, T. and N. Brekke. "Mental Contamination and Mental Correction: Unwanted Influences on Judgments and Evaluations." Psychological Bulletin, 116 (July 1994): 117. [11]

Winkler, R., J. Smith, and D. Fryback. "The Role of Informative Priors in Zero-Numerator Problems: Being Conservative Versus Being Candid." The American Statistician, 56 (2002): 1. [34]

Witmer, M., C. Margo, and M. Drucker. “Tilted Optic Disks.” Survey of Ophthalmology, 10 (2010): 403. [148]

Young v. State, 879 A.2d 44 (Md. 2005). [131]

Zabell, S. "Fingerprint Evidence." Journal of Law and Policy, 13 (2005): 143, 177, 178. [32, 124]

Zammit, K. “Computer Icons: A Picture Says a Thousand Words. Or Does It?” Journal of Educational Computing Research, 23 (2000): 217. [151]

Zhao, Q., D. Zhang, L. Zhang, et al. "High Resolution Partial Fingerprint Alignment Using PoreValley Descriptors.” Pattern Recognition, 2009. [62]

Zimring, C., M. Rashid, and K. Kampschroer. "Facility Performance Evaluation (FPE)." Whole Building Design Guide. National Institute of Building Sciences. 2010. [143] 


\section{List of Cases}

Amax Coal Co. v. Beasley, 957 F.2d 324, 328 (7th Cir.1992). [119]

Baines, United States v., 573 F.3d 979 (10th Cir. 2009). [32 (at 984, 997 - 98), 123 (at 992)]

Baker, United States v., 855 F.2d 1353, 1359 (8th Cir. 1988). [92]

Brady v. Maryland, 373 U.S. 83, 87 (1963). [91]

Briscoe v. Virginia, 130 S.Ct. 1316 (2010). [94]

Bullcoming v. New Mexico, 131 S.Ct. 2705 (2010). [93]

Bunche v. State, 5 So.3d 38, 40 n.1 (Fla. Dist. Ct. App. 2009). [127]

Carmichael v. Samyang Tire, Inc., 131 F.3d 1433, 1435-36 (1997), rev'd sub nom. Kumho Tire

Co. v. Carmichael, 526 U.S. 137 (1999). [121]

Carter v. State, 766 N.E.2d 377 (Ind. 2002). [123]

Carter, Commonwealth v., 932 A.2d 1261 (Pa. 2007). [92]

Clark, People v., 857 P.2d 1099 (Cal. 1993). [123]

Cooey v. Strickland, 589 F.3d 210, 231 (6th Cir. 2009). [119]

Cua, People v., 119 Cal.Rptr.3d 391 (Cal. Ct. App. 2011). [131]

Daubert v. Merrell Dow Pharmaceuticals, Inc., 509 U.S. 579 (1993). [22, 36 (at 594), 86, 118, 121]

Davis, United States v., 602 F.Supp.2d 658 (D.Md. 2009). [131]

Drayton, Commonwealth v., 434 N.E.2d 997 (Mass. 1982). [132]

Foreman, State v., 954 A.2d 135 (Conn. 2008). [123]

Frye v. United States, 293 F. 1013 (D.C. Cir. 1923). [86, 120]

Gambora, Commonwealth v., 933 N.E.2d 50, 56 (Mass. 2010). [130]

Glynn, United States v., 578 F.Supp.2d 567, 574-75 (S.D.N.Y. 2008). [119, 120]

Gutierrez-Castro, United States v., 86 Fed. R. Evid. Serv. 319 (D.N.M. 2011). [123]

Hartman, State v., 754 N.E.2d 1150 (Ohio 2001). [81]

Hicks v. Schurr, 871 F.2d 255 (8th Cir. 1982). [132]

Hugh, United States v., No. Crim.A. 03-829, 2009 WL 212420 (E.D. Pa. Jan. 28 2009). [130]

Kumho Tire Co. v. Carmichael, 526 U.S. 137, 141, 154 (1999). [121]

Kyles v. Whitley, 514 U.S. 419, 437 (1995). [91]

Lewicki v. Longshore, No. 17004/06, 2009 WL 4667411, at 2 (N.Y. Sup. Ct. Dec. 7, 2009). [119]

Llera Plaza, United States v., Crim. No. 98-362-10, 11, 12, 2002 U.S. Dist. LEXIS 344 (E.D. Pa.

Jan. 7, 2002), vacated, 188 F. Supp. 2d 549 (E.D. Pa. 2002). [32, 122, 123, 130]

McClanahan, People v., 729 N.E.2d 470, 472 (Ill. 2000). [92]

McDaniel, People v., 670 N.W.2d 659 (Mich. 2003). [92]

McGrew, State v., 682 N.E.2d 1289 (Ind. 1997). [136]

McVeigh, United States v., No. 96-CR-68 (D. Colo. May 5, 1997) (transcript). [137]

McWhorter, People v., 212 P.3d 692 (Cal. 2009). [81]

Melendez Diaz v. Massachusetts, 129 S. Ct. 2527, 2535 (2009). [93]

Mitchell, United States v., 199 F. Supp. 2d 262 (E.D. Pa. 2002) (No. 96-407). [32]

Monteiro, United States v., 407 F.Supp.2d 351, 375 (D.Mass. 2006). [120]

Nichols, United States v., No. 96-CR-68 (D. Colo. Nov. 10, 1997) (transcript). [137]

Oates, United States v., 560 F.2d 45 (2d Cir. 1977). [92]

Pendergrass v. State, 913 N.E.2d 703, 709 (Ind. 2009). [92]

Pierce v. Gilchrist, 359 F.3d 1279 (10th Cir. 2004). [91]

Proctor, State v., 595 S.E.2d 480 (S. Car. 2004). [127] 
Pytou Heang, Commonwealth v., 942 N.E.2d 927 (Mass. 2011). [120, 122]

R. v. Smith, 2011 EWCA Crim 1296. [vi]

Reid, State v., 757 A.2d 482, 487 (Conn. 2000). [123]

Rose, State v., No. K06-0545 (Md. Cir. Ct. Oct. 19, 2007). [130]

Swinton, State v., 847 A.2d 921 (Conn. 2004). [81]

Taylor, United States v., 663 F.Supp.2d 1170, 1180 (D.N.M. 2009). [120, 122]

Tharpe, United States v., 38 M.J. 8, 28 (C.M.A. 1993). [116]

Tiscareno v. Anderson, 639 F.3d 1016 (10th Cir. 2011), vacated in part, 421 Fed. Appx. 842 (10th Cir. 2011). [91]

Warney v. Monroe County, 587 F.3d 113, 118 (2d Cir. 2009). [118]

Williams v. State, 679 A.2d 1106 (Md. 1996). [126]

Williams, People v., 939 N.E. 2d 268, 270 (Ill. 2010), cert. granted sub nom. Williams v. Illinois, 80 U.S.L.W. 3003 (U.S. June 28, 2011). [93]

Willock, United States v., 696 F.Supp.2d 536 (D.Md. 2010). [91 (at 570), 119 (at 574), 122]

Young v. State, 879 A.2d 44 (Md. 2005). [131]

Zajac, United States v., No. 2:06-cr-00811-CW (D. Utah Sept. 16, 2010). [120, 130, 137] 
